\title{
Measurement of the Elastic Ep Cross Section at Q2 = 0.66, 1.10, 1.51 and 1.65 Gev2
}

\author{
YANG WANG \\ College of William and Mary, 466929938@qq.com
}

Follow this and additional works at: https://scholarworks.wm.edu/etd

Part of the Nuclear Commons

\section{Recommended Citation}

WANG, YANG, "Measurement of the Elastic Ep Cross Section at Q2 $=0.66,1.10,1.51$ and 1.65 Gev2" (2017). Dissertations, Theses, and Masters Projects. Paper 1516639568.

http://dx.doi.org/doi:10.21220/S29H4J

This Dissertation is brought to you for free and open access by the Theses, Dissertations, \& Master Projects at W\&M ScholarWorks. It has been accepted for inclusion in Dissertations, Theses, and Masters Projects by an authorized administrator of W\&M ScholarWorks. For more information, please contact scholarworks@wm.edu. 
Measurement of the Elastic ep Cross Section at $Q^{2}=0.66,1.10,1.51$ and $1.65 \mathrm{GeV}^{2}$

\author{
Yang Wang \\ Jiangsu, China
}

Master of Science, College of William \& Mary, 2012

Bachelor of Science, University of Science and Technology of China, 2010

A Dissertation presented to the Graduate Faculty of College of William \& Mary in Candidacy for the Degree of Doctor of Philosophy

Department of Physics

College of William \& Mary

August 2017 
(C) 2017

Yang Wang

All rights reserved. 


\section{APPROVAL PAGE}

This Dissertation is submitted in partial fulfillment of the requirements for the degree of

Doctor of Philosophy

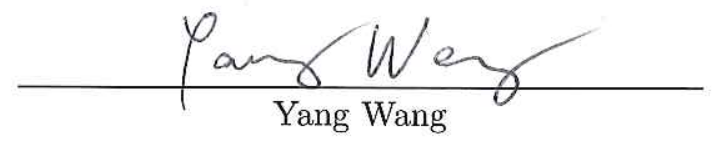

Approved by the Committee, June; 2017

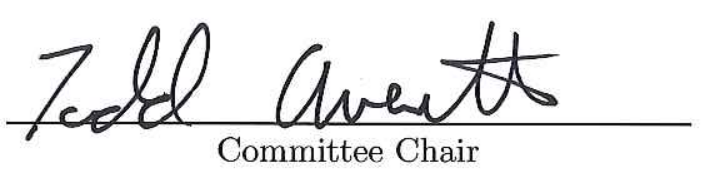

Professor Todd Averett, Physics

College of William \& Mary

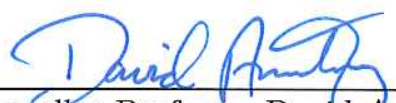

Chancellor Professor David Armstrong, Physics

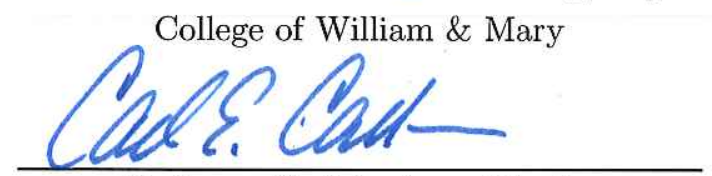

Professor Carl Carlson, Physics

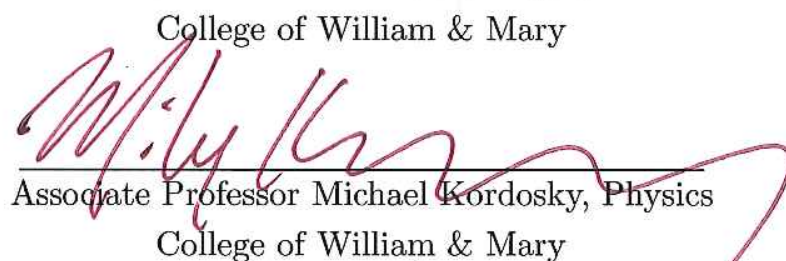

College of William \& Mary

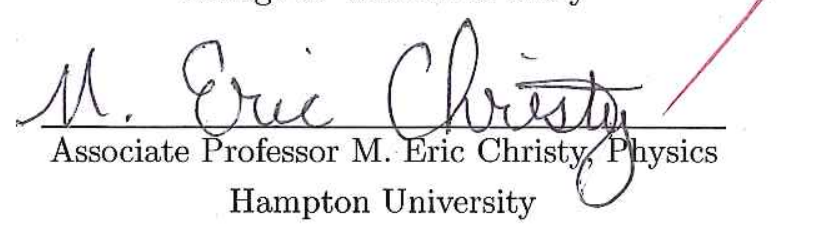




\begin{abstract}
The nucleon form factors have been investigated by physicists for decades because of their fundamental importance. The world data of the proton magnetic form factor $G_{M p}$ has been focused on $Q^{2}$ lower than $5 \mathrm{GeV}^{2}$ and they have large uncertainties at higher $Q^{2}$. Jefferson Lab experiment E12-07-108 aims to improve the accuracy of the $e-p$ elastic cross section to better than $2 \%$ over a $Q^{2}$ range of $7-14 \mathrm{GeV}^{2}$. From 2015 to 2016, the $e-p$ elastic cross section was measured over a wide range of $Q^{2}$ from $0.66-$ 12.56 $\mathrm{GeV}^{2}$ at the Thomas Jefferson National Accelerator Facility in Virginia, USA. An unpolarized electron beam was scattered off a cryogenic hydrogen target and the scattered electron was detected in the high resolution spectrometers. This thesis focuses on the cross section calculations of the data taken in the spring of 2015, where $Q^{2}=$ $0.66,1.10,1.51$ and $1.66 \mathrm{GeV}^{2}$. At $Q^{2}=0.66 \mathrm{GeV}^{2}$, an uncertainty $<3 \%$ was achieved and $<5 \%$ was achieved for the other three $Q^{2}$ at the moment. The results were compared with the world data and the good agreement provides confidence for the experimental measurements at higher $Q^{2}$.
\end{abstract}




\section{TABLE OF CONTENTS}

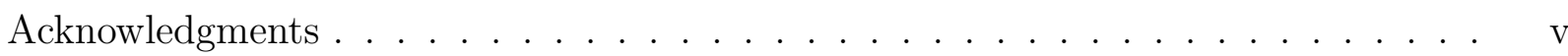

List of Tables . . . . . . . . . . . . . . . . . . . . . vi

List of Figures . . . . . . . . . . . . . . . . . . . . vii

CHAPTER

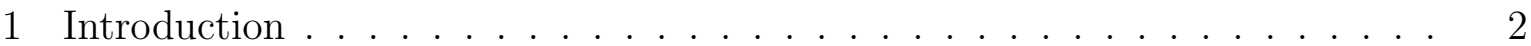

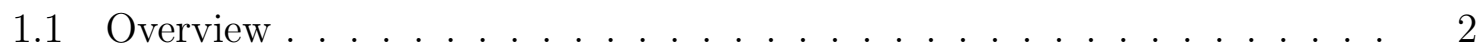

1.2 Electron Scattering . . . . . . . . . . . . . . . . 4

1.3 Form Factors . . . . . . . . . . . . . . . . . . 6

1.4 Rosenbluth Separation Method ................ 10

1.5 Recoil Polarization Method . . . . . . . . . . . . . . . . . 12

1.6 Existing Measurements . . . . . . . . . . . . . . . . . . . . . 14

2 Physics of Nucleon Form Factors . . . . . . . . . . . . . . . . 20

2.1 Vector Meson Dominance and Dispersion Relations . . . . . . . . . . 20

2.1.1 Vector Meson Dominance . . . . . . . . . . . . . 20

2.1.2 Dispersion Relations . . . . . . . . . . . . . . . . . . 22

2.2 Constituent Quark Models . . . . . . . . . . . . . . . 24

2.3 Perturbative $(\mathrm{pQCD}) \ldots \ldots \ldots \ldots \ldots$

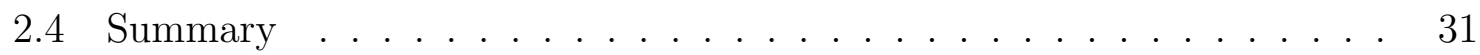

3 Experimental Setup . . . . . . . . . . . . . . . . . 33

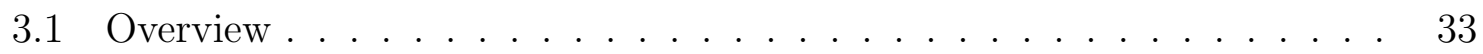

3.2 Accelerator and Beam Line Components . . . . . . . . . . . . . 36 
3.2.1 Continuous Electron Beam Accelerator Facility . . . . . . . . . 36

3.2 .2 Hall A . . . . . . . . . . . . . . . . . . . . . . . . 38

3.2.3 Beam Energy Measurement . . . . . . . . . . . . . . . . . . 39

3.2.4 Beam Position and Direction . . . . . . . . . . . . . . . 41

3.2.5 Beam Current Measurement . . . . . . . . . . . . . . . . 42

3.2.6 Hall A Cryogenic Target System . . . . . . . . . . . . . . . . 44

4 High Resolution Spectrometers (HRSs) _ . . . . . . . . . . . . . . 46

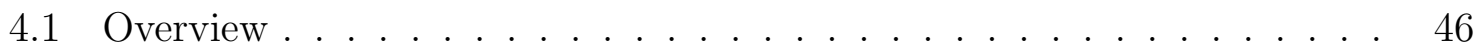

4.2 Detector Packages . . . . . . . . . . . . . . . . . . 48

4.2 .1 Vertical Drift Chambers (VDCs) . . . . . . . . . . . . 48

4.2 .2 Straw Chamber $(\mathrm{SC}) \ldots \ldots \ldots \ldots \ldots$

4.2 .3 Plastic Scintillators and Triggers . . . . . . . . . . . . . 54

4.2 .4 Gas Cherenkov Detector . . . . . . . . . . . . . . 57

4.2 .5 Electromagnetic Calorimeter . . . . . . . . . . . . . 58

4.2 .6 Data Acquisition $(\mathrm{DAQ}) \ldots \ldots \ldots \ldots$

4.3 Spectrometer Optics _. . . . . . . . . . . . . . . . . . 60

4.3 .1 Coordinate Systems . . . . . . . . . . . . . . . . . . 60

4.3.2 Optimization Method . . . . . . . . . . . . . . . 63

5 GMp High Resolution Spectrometer (HRS) Improvement \& Calibration . . . 65

5.1 Detector Packages Improvement _ . . . . . . . . . . . . . 65

5.1 .1 VDC Improvement . . . . . . . . . . . . . . . . 65

5.1 .2 Straw Chamber Improvement $\ldots \ldots \ldots \ldots$

5.2 GMP optics . . . . . . . . . . . . . . . . . . . . . 82

5.2 .1 New Sieve for GMp Optics . . . . . . . . . . . . . . 83

$5.2 .2 \theta_{t g}$ and $\phi_{t g}$ Optimization $\ldots \ldots \ldots \ldots \ldots$ 
$5.2 .3 y_{t g}$ Optimization $\ldots \ldots \ldots \ldots \ldots \ldots \ldots$

$5.2 .4 \quad \frac{d P}{P}(\delta)$ Optimization . . . . . . . . . . . . . . 94

6 Data Analysis . . . . . . . . . . . . . . . . . . . . . . . . . . 97

6.1 Analysis Introduction . . . . . . . . . . . . . . . . . 97

6.2 Event Reconstruction . . . . . . . . . . . . . . . . . . . . . . . . . . . . 98

6.3 VDC tracking Efficiency $\ldots \ldots \ldots \ldots$

6.4 Trigger Efficiency . . . . . . . . . . . . . . . . . . 100

6.5 Particle Identification Efficiency (PID) . . . . . . . . . . . . 100

6.5.1 Cherenkov . . . . . . . . . . . . . . . . . . . . . . 101

6.5 .2 Cut Efficiency . . . . . . . . . . . . . . . . 102

6.5 .3 Electromagnetic Calorimeter . . . . . . . . . . . . . . 103

6.5 .4 Cut Efficiency . . . . . . . . . . . . . . . . . 105

6.5 .5 Pion Suppresion . . . . . . . . . . . . . . . . 107

6.6 Computers and Electronics Deadtime . . . . . . . . . . . . . 108

6.7 Target Boiling Correction . . . . . . . . . . . . . . . . . . . . . . 109

6.8 Monte-Carlo Simulations . . . . . . . . . . . . . . . . . . . . . . . 110

6.9 Radiative Corrections . . . . . . . . . . . . . . . . . . . . . . . . . 113

6.10 Cryogenic Target Aluminum Cell _. . . . . . . . . . . . . . 118

6.11 Summary of the Uncertainties . . . . . . . . . . . . . . . . 120

7 Results . . . . . . . . . . . . . . . . . . . . . . . . . . . 121

7.1 Reduced Cross Section $\sigma_{R}$ Extraction $\ldots \ldots \ldots . \ldots 121$

7.2 Comparison with World Data . . . . . . . . . . . . . . 128

7.3 Conclusion . . . . . . . . . . . . . . . . . . . . 133

APPENDIX A

Diagrams of the tungsten sieve for GMp optics calibration . . . . . . . . 136 


\section{APPENDIX B}

MC Uncertainty . . . . . . . . . . . . . . . . . . . . . . . . . . . . . 139

Bibliography . . . . . . . . . . . . . . . . . . . . . . 141 


\section{ACKNOWLEDGMENTS}

At the beginning of my thesis, I want to express my appreciation to the people who gave me so much support and help during my Ph. D. study. Without them, this thesis would not have been possible.

First, I would like to thank my academic advisor, Prof. Todd Averett. I am very lucky that he gave me the opportunity to pursue the Ph. D. degree at W\&M. He couldn't be more supportive and encouraging. I benefited so much from his knowledge of physics and guidance in academic research. His valuable instructions through my $\mathrm{Ph}$. D. study helped me to get a clear picture of both the physics and research. I also benefited a lot from his cheerfulness. Every time when I felt frustrated, I became motivated and confident again after talking with him. I am extremely grateful to have him as my Ph.D. advisor.

I also would like to thank my co-academic advisor Prof. Charles Perdrisat, who introduced me to the field of experimental nuclear physics when I had no experience. His knowledge of nucleon form factors benefited me so much and I really appreciate his patience, support and guidance.

I joined the E08-07-108 collaboration at Jefferson Lab in 2013 and I learned a lot from working with the spokespersons, post-docs and graduate students. I would like to thank Dr. Bogdan Wojtsekhowski for his guidance and supervision during my experimental research. I learned how to be an experimental physicist from his enthusiasm and rigorous attitude for physics. I would like to thank Prof. M. Eric Christy for the valuable suggestions and comments on the experimental data analysis. His guidance made this thesis possible. I also would like to thank post-doc Vincent Sulkosky and Kalyan Allada. They generously shared their experience of both the experimental setup and data analysis with me.

I had a wonderful experience working with the students of the E08-07-108 collaboration: Thir Narayan Gautam, Longwu Ou and Barak Schmookler. This thesis is based on their contribution to the calibrations of the experimental setup, monte carlo simulations and data analysis. Thanks for Longwu's patience when I bothered him with all kinds of "dumb" questions.

Finally, I would like to express the deepest appreciation to my parents. Thanks for giving me not only guidance and support but also freedom of choice. I feel extremely lucky to be your daughter. 


\section{LIST OF TABLES}

1.1 Quark families in the Standard Model, where Q is charge. . . . . . . . . 3

3.1 List of the targets on the ladder of Hall A for experiment E12-07-108. . . . 44

5.1 List of the pulse widths of the 8 multiplexed signals in one channel of SC. . 82

5.2 List of the horizontal offset of each sieve hole for each foil target. . . . . . . 87

5.3 List of the vertical offset of each sieve hole for each foil target. . . . . . . . 87

6.1 Summary of the kinematics in the spring of 2015 for experiment E12-07-18. 98

6.2 The VDC tracking efficiency for each run. . . . . . . . . . . . . . . . . 99

6.3 Cut efficiency of Cherenkov on both spectrometer arms. . . . . . . . . . . . 104

6.4 PID cuts summary ... . . . . . . . . . . . . . . 107

6.5 Pion contamination on both spectrometer arms with the three cuts mentioned above applied. . . . . . . . . . . . . . . . 107

6.6 The computer and electronics deadtime for each kinematics. . . . . . . . . 109

6.7 The average beam current $I_{\text {beam }}$ and target boiling correction $C_{T B}$ for each kinematics. . . . . . . . . . . . . . . . . . 110

6.8 The radiative correction $\delta_{\text {corr }}$ in SIMC for each kinematics, where the cut of the invariant mass $\mathrm{W}<1.07 \mathrm{GeV}$ is applied. . . . . . . . . . . . . . 117

6.9 The dummy contribution for each kinematics. . . . . . . . . . . . . . . . 118

6.10 The summary of the uncertainties in the extracted cross section for each kinematics. . . . . . . . . . . . . . . . . . 120

7.1 The elastic $e-p$ reduced cross section $\sigma_{R}$ for all the kinematics. . . . . . 128

B.1 The uncertainties calculated by the MC when the field strength is off the setting value for each quadrupole. . . . . . . . . . . . . . . . . . 139

B.2 The uncertainties caused by the displacement of each aperture. . . . . . . . 140 


\section{LIST OF FIGURES}

1.1 Feynman digram for lowest-order inclusive electron scattering. . . . . . . . 4

1.2 Feynman digram for lowest order elastic electron-proton scattering. . . . . 6

1.3 Demonstration of the Rosenbluth separation method based on the data from [1]. $Q^{2}=2.5 \mathrm{GeV}^{2} \ldots \ldots \ldots \ldots \ldots 11$

1.4 Schematic diagram for polarization transfer from a longitudinally polarized electron to a proton with exchange of a virtual photon. . . . . . . . . . . 13

1.5 Proton electric form factor $G_{E p}$ data obtained using the Rosenbluth separation method. . . . . . . . . . . . . . . . . . . . . . .

1.6 Proton magnetic form factor $G_{M p}$ data obtained using the Rosenbluth separation method. . . . . . . . . . . . . . 16

1.7 Proton electric and magnetic form factor ratio $\mu_{p} G_{E p} / G_{M p}$ obtained with both the Rosenbluth separation and polarization techniques. . . . . . . . . 17

1.8 Ratio $\mu_{p} G_{E p} / G_{M p}$ extracted from polarization transfer (filled diamonds) and Rosenbluth method (open circles). . . . . . . . . . . . . . . 19

2.1 Feynman diagram of the electron nucleon scattering in the VMD model . .

2.2 The proton form factor ratio $\mu_{p} G_{E p} / G_{M p}$ data from Jefferson Lab Hall A with various VMD model fitting results. . . . . . . . . . . . . 23

2.3 Dispersion relation for the nucleon (space-like) form factors. . . . . . . . . 24

2.4 Comparison of relativistic CQM calculations with data for $\mu_{p} G_{E p} / G_{M p}$. . 27

2.5 Two typical Feynman diagrams contributing to $e N \rightarrow e N$ at leading order in $\alpha_{s}$ in pQCD. . . . . . . . . . . . . . . . . . . . . . . 29

$2.6 Q^{4} G_{M p} / \mu_{p}$ vs. $Q^{2} \ldots \ldots \ldots \ldots \ldots$

2.7 Test of the modified scaling prediction for $F_{2 p} / F_{1 p}$ of Ref. [2] . . . . . . . 32

3.1 The $Q^{2}$ measured by experiment E12-07-108 shown with the $e-p$ elastic cross sections obtained by the analysis in this thesis and previous measure-

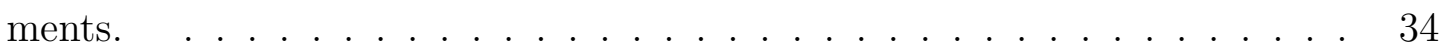

3.23 -D model of the E12-07-108 experimental apparatus. . . . . . . . . . . . 35

3.3 Sketch of the CEBAF accelerator. . . . . . . . . . . . . . . . 37

3.4 Floor plan during experiment E12-07-108. . . . . . . . . . . . . . 38 
3.5 Schematic of the arc energy measurement. . . . . . . . . . . . . . 40

3.6 Layout of Hall A beamline components for experiment E12-07-108. . . . . 42

3.7 The photograph of the target ladder inside the scattering chamber in the

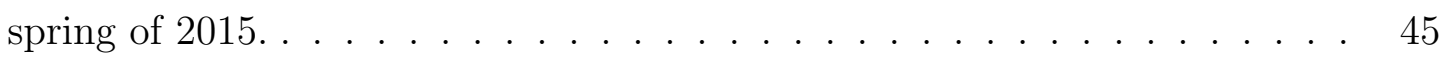

4.1 Schematic of Hall A High Resolution Spectrometer and the detector package for the E12-07-108 experiment. . . . . . . . . . . . . . . 47

4.2 Schematic layout of the VDCs (not to scale). . . . . . . . . . . . 49

4.3 Configuration of the wire chambers in the VDCs [3] and a typical 5-cell track. 51

4.4 The coordinate system of the straw chamber. . . . . . . . . . . . . 52

4.5 The schematic layout of the three U planes of tubes for SC. . . . . . . . . 53

4.6 Tubes configuration in three $\mathrm{U}$ planes or $\mathrm{V}$ planes in the straw chamber. . 54

4.7 Scintillator $\mathrm{S}_{0}$ plane configuration. . . . . . . . . . . . . . 55

4.8 Scintillator $\mathrm{S}_{2 m}$ plane configuration. . . . . . . . . . . . . 56

4.9 Schematic layout of part of the shower detectors in HRS-L (top) and HRS-R (bottom) . . . . . . . . . . . . . . . . . . . 59

4.10 A simple CODA implementation. [4]. . . . . . . . . . . . . . . . 59

4.11 Target coordinate system (TCS) for a electron scattering from a thin foil

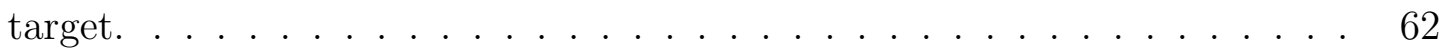

5.1 Block diagram for the logic of the VDC signal. . . . . . . . . . . . . 66

5.2 Photograph of the MAD cards. . . . . . . . . . . . . . . . 67

5.3 TDC value differences between adjacent wires of the third MAD card on the $\mathrm{U} 2$ plane of VDCs. . . . . . . . . . . . . . . . . . . . 69

5.4 The wire efficiencies for 4 VDCs planes of Right-arm HRS after 26 MAD cards with low efficiencies were replaced. . . . . . . . . . . 71

5.5 Relative timing $\Delta T$ (see Eq. 5.2) for 4 planes of VDCs on the Right-arm HRS with HV being $-3.5 \mathrm{kV} \ldots \ldots \ldots \ldots \ldots \ldots$

5.6 Photograph of one level translator card designed for the MAD cards. . . . 74

5.7 Photograph of the level translator and its power supply. . . . . . . . . . 74

5.8 Photograph of one SC during modification before experiment 12-07-108. . . 75

5.9 Block diagram for the logic of the SC signal. . . . . . . . . . . . . 76

5.10 Photograph of the new HV distribution board and new gas distribution

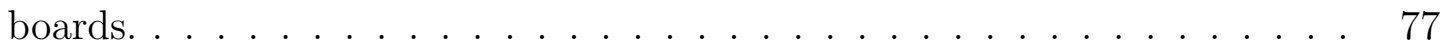

5.11 Photograph of the magnehelic gauge and gas valves at the side of the SC. . 78

5.12 Old and new gas distribution systems for each subgroup of tubes. . . . . . 79 
5.13 Photograph of the gas output pipes for the SC of the U planes . . . . . . . 80

5.14 Scheme shows a electron scattering at the multi-foil target goes through a sieve slit hole. . . . . . . . . . . . . . . . . . 83

$5.15 \theta_{t g}$ vs $\phi_{t g}$ with different sieves. . . . . . . . . . . . . . . . . . . . 85

5.16 Scheme showing the offset from the hole center to the average position distributions caused by the thickness of the sieve. . . . . . . . . . 86

5.17 The reconstructed positions at the sieve plane after the $\theta_{t g}$ and $\phi_{t g}$ optimizations. . . . . . . . . . . . . . . . 88

5.18 The position distribution in the horizontal direction with the Gaussian fittings of the middle row of the sieve holes for the middle foil target. . . . . 89

5.19 The reconstructed positions at the sieve plane after the $\theta_{t g}$ and $\phi_{t g}$ optimizations with pseudo data produced by the $\mathrm{MC}$ are included. . . . . . . . 91

5.20 The comparison of $\theta_{t g}$ and $\phi_{t g}$ between the MC and experimental data of kinematics $b$ (see Table 6.1) . . . . . . . . . . . . . . . . . . 92

5.21 The $y_{t g}$ optimization results which shows the Gaussian fitting mean values of the reconstructed foil target positions compared with the real ones. . . . 93

5.22 Three cases for the electron scattering in the liquid hydrogen target. . . . . 94

5.23 The $\frac{d P}{P}$ optimization results (see text). . . . . . . . . . . . . . 95

6.1 Plots of the electron detecting efficiency of the Cherenkov detector. . . . . 101

6.2 Plots of the electron detecting and pion rejecting efficiencies of the Cherenkov detector. . . . . . . . . . . . . . . . . 103

6.3 The Cherenkov electron detecting and pion rejecting efficiencies vs. Cherenkov cut. . . . . . . . . . . . . . . . . 104

6.4 Plots of the electron detecting efficiency of the shower. . . . . . . . . . 105

6.5 Plots of the electron detecting efficiency of the shower and pre-shower. . . 106

6.6 Target boiling study. . . . . . . . . . . . . . . . . . . . 110

6.7 Born term and lowest order radiative correction graphs for the electron in

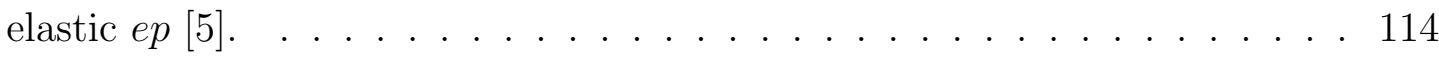

6.8 Lowest order radiative correction graphs for the proton in elastic ep [5]. . . 116

6.9 The liquid hydrogen $\mathrm{LH}_{2}$ and dummy target data yields for kinematics a. . 119

7.1 Data yield compared to Monte Carlo yield of kinematics a (see Table 6.1). 124

7.2 Data yield compared to Monte Carlo yield of kinematics $b$ (see Table 6.1) . 125

7.3 Data yield compared to Monte Carlo yield of kinematics $c$ (see Table 6.1). 126

7.4 Data yield compared to Monte Carlo yield of kinematics d (see Table 6.1). 127 
7.5 The $e-p$ elastic cross sections obtained from the experiment E12-07-108 data in the spring of 2015 at $Q^{2}=0.66,1.10,1.51,1.65 \mathrm{GeV}^{2} \ldots \ldots \ldots 129$

7.6 Comparison of the $e-p$ elastic cross sections between the experiment E1207-108 results and previous measurements. . . . . . . . . . . 130

7.7 The reduced elastic $e-p$ cross sections vs. $\epsilon$ obtained from previous measurements at $Q^{2}$ close to $0.66 \mathrm{GeV}^{2}$ and the experiment E12-07-108 data at

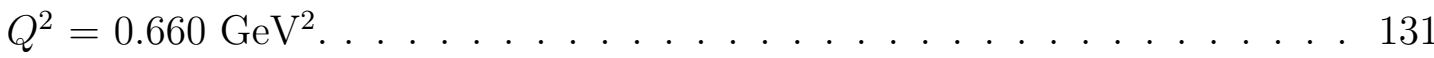

7.8 The reduced elastic $e-p$ cross sections vs $\epsilon$ obtained from previous measurements at $Q^{2}$ close to $1.099 \mathrm{GeV}^{2}$ and the experimental data at $Q^{2}=$ $1.099 \mathrm{GeV}^{2} \ldots \ldots \ldots \ldots \ldots \ldots \ldots \ldots$

7.9 The comparison of the proton electric form factor $G_{E p}$ between the world data and that obtained by combining the experimental data and previous measurements at similar $Q^{2} \ldots \ldots \ldots \ldots$. . . . . . . . . 133

7.10 The comparison of the proton electric form factor $G_{M p}$ between the world data and that obtained by combining the experiment E12-07-108 data and previous measurements at similar $Q^{2} \ldots \ldots \ldots \ldots \ldots$ 
MEASUREMENT OF THE ELASTIC $e p$ CROSS SECTION AT $Q^{2}=0.66,1.10,1.51$ AND 1.65 $\mathrm{GEV}^{2}$ 


\section{CHAPTER 1}

\section{Introduction}

\section{$1.1 \quad$ Overview}

The nucleons, which are the building blocks of the nucleus, play a role of fundamental importance in physics. Understanding the internal structure of the nucleons has been of considerable interest to physicists and decades of efforts have been devoted to investigating it.

In 1911, Ernest Rutherford did the gold foil experiment and discovered that the atom must have a concentrated nucleus which contains positive charge and most of the atom's mass. He named the positive constituent of the nucleus "proton" and suggested the neutrally-charged constituent is a bound proton and electron. In 1932, James Chadwick performed a series of experiments and showed that the nucleus is composed of protons and neutrons, which are uncharged particles with about the same mass as the proton. At that time, the protons and neutrons were believed to be Dirac particles, just like the electrons. In 1933 Otto Stern measured the magnetic moment of the proton and found

it close to $2.79 \mu_{N}\left(\mu_{N}=\frac{e \hbar}{2 M_{p}}\right)$. This value is dramatically different from $0.5 \mu_{N}$, which is 
expected for a point-like spin 1/2 charged particle. The magnetic moment of the neutron was measured soon after and found close to $-1.91 \mu_{N}$, also very different from the $0.0 \mu_{N}$ that a neutral Dirac particle would have. These discoveries imply that the protons and neutrons are composite and have internal structure, instead of being elementary particles.

The Standard Model of elementary particles and interactions has been developed throughout the latter half of the 20th century, in which the protons and neutrons are composed of elementary fermions called quarks and are held together by the strong force, mediated by gluons. There are six different types of quarks, known as flavors: u(up), $\mathrm{d}($ down $), \mathrm{s}$ (strange), $\mathrm{c}($ charm $), \mathrm{t}($ top $)$, and b(bottom). The quarks are grouped into three families as shown in Table 1.1. Each family has one member with charge $+\frac{2}{3} \mathrm{e}$ and one member with charge $-\frac{1}{3} \mathrm{e}$. The proton is composed of 2 up quarks and 1 down quark and the neutron is composed of 1 up quark and 2 down quarks. The theory of Quantum Chromodynamics (QCD) describes the strong interaction, which is responsible for binding quarks through the exchange of gluons to form hadrons (baryons and mesons). The coupling strength between the quarks and gluons is strong and increases at large distances. In the short distance scale, the interactions between quarks are weak and they behave almost like they are free. In the large distance scale, the interactions are strong and thus hadrons form. This explains why no free quarks have been observed experimentally. Only hadrons are found in nature.

TABLE 1.1: Quark families in the Standard Model, where Q is charge.

\begin{tabular}{|l|l|l|l|}
\hline $\mathrm{Q}=+\frac{2}{3} \mathrm{e}$ & $\mathrm{u}$ & $\mathrm{c}$ & $\mathrm{t}$ \\
\hline $\mathrm{Q}=-\frac{1}{3} \mathrm{e}$ & $\mathrm{d}$ & $\mathrm{s}$ & $\mathrm{b}$ \\
\hline
\end{tabular}




\subsection{Electron Scattering}

Electron scattering is one of the most powerful techniques to study the underlying structure of the nucleon. When scattered by a target nucleus, the electron exchanges virtual photons, which probe the electromagnetic structure of the nucleus. The electron is an elementary particle without internal structure and thus the differential scattering cross section only carries the information of the nucleus without contribution from the electron. This makes the electron a clean probe of the target nucleus.

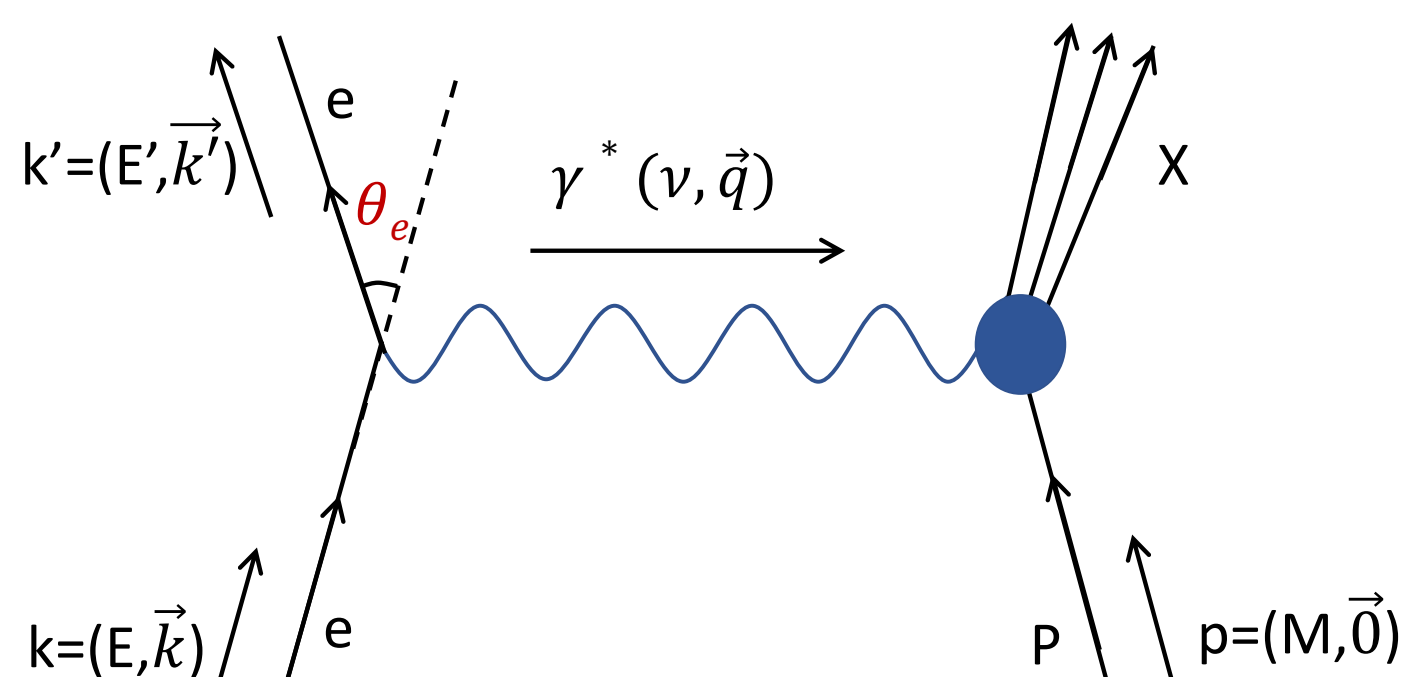

FIG. 1.1: Feynman digram for lowest-order inclusive electron scattering.

The electron-photon vertex is well understood within the theory of Quantum Electrodynamics (QED). To the lowest-order of the electromagnetic coupling constant $\alpha$, the scattering diagram looks like Fig. 1.1, where the electron of initial four momentum $k^{\mu}=(E, \vec{k})$ exchanges a single virtual photon $\gamma^{*}$ with a nucleon of initial four momentum $p$. Because $\alpha$ is small ( $\alpha \simeq 1 / 137)$, it is a very good approximation to the real physics process when considering only the first order (Born approximation). The scattering angle is $\theta_{e}$ and the scattered electron four momentum is $k^{\prime \mu}=\left(E^{\prime}, \overrightarrow{k^{\prime}}\right)$. In electron scattering experiments, the 
electron with known momentum $k^{\mu}$ scatters off a stationary target, thus $p^{\mu}=(M, \overrightarrow{0})$. For inclusive scattering, the final hadronic system $\mathrm{X}$ goes undetected. The exchanged virtual photon carries four-momentum $q^{\mu}=(\nu, \vec{q})=\left(k-k^{\prime}\right)^{\mu}$ and transfers an energy $\nu=\frac{P \cdot q}{M}$ and three-momentum $\vec{q}$ to the target.

Typically the scattering process is described with two main variables, the squared fourmomentum transfer $Q^{2}=-q^{2}$ or the invariant mass of the residual hadronic system $W$. Highly relativistic electrons are used in electron scattering experiment, thus the electron mass is neglected since $E$ and $E^{\prime} \gg m_{e}$. The relevant variables are defined as follows:

$$
\begin{gathered}
\nu=E-E^{\prime} \\
Q^{2}=-q^{2}=4 E E^{\prime} \sin ^{2} \frac{\theta}{2} \\
W=\sqrt{(P+q)^{2}}=M^{2}+2 M \nu-Q^{2}
\end{gathered}
$$

When investigating inclusive scattering, it is usually separated into three kinematics regions: elastic, quasi-elastic and inelastic; inelastic includes both resonance production and deep inelastic scattering. At low energy transfers, elastic scattering dominates, in which the nucleon (or nulceus) remains in its ground state after the scattering and the energy and momentum transfer are absorbed by the recoil nucleon. For elastic scattering, the invariant mass $W$ is equal to the mass of the nucleon or nucleus so that $\nu=\frac{Q^{2}}{2 M}$. As the energy transfer increases and becomes larger than the nuclear binding energy, we enter the quasi-elastic region. The electron knocks a single nucleon out of the nucleus, which can be considered as quasi-free. Compared to the elastic scattering, the quasi-elastic peak is shifted due to the nuclear binding energy and broadened due to the Fermi motion of the nucleons inside the nucleus. By increasing $Q^{2}$ and the energy transfer further, we enter the region of the nucleon resonance. The quarks inside the nucleon absorb the virtual photons and form excited states which we refer to as nucleon resonances. If we increase $Q^{2}$ and the 
energy transfer further, we enter the deep inelastic region (DIS). DIS is typically defined as $Q^{2}>1-2 \mathrm{GeV}^{2}$ and $W>2 \mathrm{GeV}$. In this case, the wavelength of the virtual photon is small compared to the size of the target hadron and thus the internal structure of the nucleon becomes "visible". The electron is primarily interacting with a single quark through the virtual photon $\gamma^{*}$.

\subsection{Form Factors}

The elastic electron-proton scattering diagram to lowest order is shown as Fig. 1.2. Due to the internal structure of the proton, the photon-proton vertex in Fig. 1.2 and two $Q^{2}$ dependent functions that contain all the information about the internal structure of the proton are introduced.

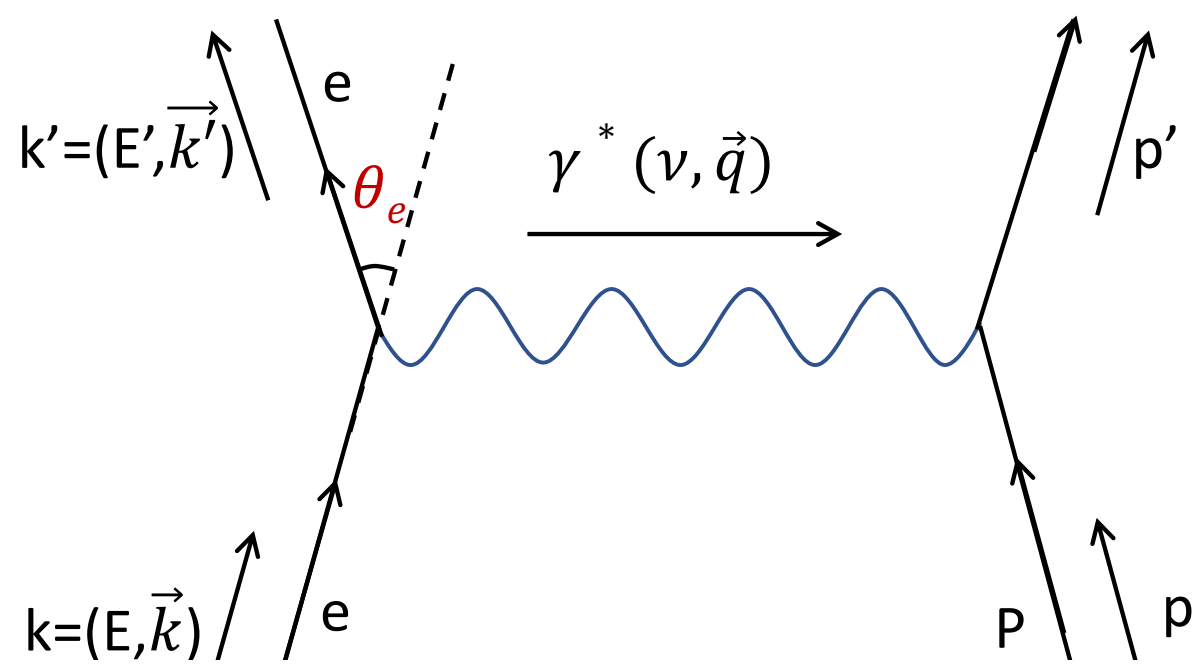

FIG. 1.2: Feynman digram for lowest order elastic electron-proton scattering.

The point-like electron has the simple vertex function $\gamma_{\mu}$ and we write the proton vertex function as $\Gamma_{\mu}$. Both of them are Dirac $4 \times 4$ matrices. The electromagnetic 
currents, $j_{\mu}$ for the electron and $J_{\mu}$ for the proton, can be expressed as follows:

$$
\begin{gathered}
j_{\mu}=-e \bar{u}\left(k^{\prime}\right) \gamma_{\mu} u(k) \\
J_{\mu}=e \bar{v}\left(p^{\prime}\right) \Gamma_{\mu} v(p)
\end{gathered}
$$

where $u(k), \bar{u}\left(k^{\prime}\right), v(p)$ and $\bar{v}(p)^{\prime}$ are the four-component Dirac spinors for the incoming and scattered electron and proton. Therefore, the amplitude for $e-p$ scattering can be written as:

$$
\begin{aligned}
i M & =\left[i e \bar{v}\left(p^{\prime}\right) \Gamma^{\mu}\left(p^{\prime}, p\right) v(p)\right] \frac{-i g_{\mu \nu}}{q^{2}}\left[i e \bar{u}\left(k^{\prime}\right) \gamma^{\nu} u(k)\right] \\
& =\frac{-i}{q^{2}}\left[i e \bar{v}\left(p^{\prime}\right) \Gamma^{\mu}\left(p^{\prime}, p\right) v(p)\right]\left[i e \bar{u}\left(k^{\prime}\right) \gamma_{\mu} u(k)\right]
\end{aligned}
$$

The proton current $\Gamma_{\mu}$ contains all the information about the internal structure of the proton. In general, $\Gamma_{\mu}$ is some expression that involves $p, p^{\prime}, \gamma^{\mu}$, and constants such as $m_{p}, e$, and pure numbers. The parity-conserving theory excludes the possibablity of $\gamma^{5}$. To lowest order, $\Gamma^{\mu}=\gamma^{\mu}$ and the proton is treated as a point-like particle. Since $\Gamma^{\mu}$ transforms as a vector, it must be a linear combination of $\gamma^{\mu}, p^{\mu}$, and $p^{\prime \mu}$ as follows:

$$
\Gamma^{\mu}=A \gamma^{\mu}+B\left(p^{\prime \mu}+p^{\mu}\right)+C\left(p^{\prime \mu}-p^{\mu}\right)
$$

where the coefficients $A, B$, and $C$ could involve Dirac matrices dotted into vectors, $\not p$ and $\not p^{\prime}$. Since $\not p v(p)=m v(p)$ and $\bar{v}\left(p^{\prime}\right) \not p^{\prime}=\bar{v}\left(p^{\prime}\right) m$, the coefficients can be written as ordinary numbers and they must be functions only of $q^{2}$ and constants such as $M_{p}$ because $q^{2}$ is the only nontrivial scalar available. Applying the Ward identity $q_{\mu} \Gamma^{\mu}$ to Eq. 1.7, the second 
term vanishes as follows:

$$
\begin{aligned}
q_{\mu} \Gamma^{\mu}= & \left(p_{\mu}-p_{\mu}^{\prime}\right) \Gamma^{\mu} \\
= & A\left(p_{\mu}-p_{\mu}^{\prime}\right) \gamma^{\mu}+B\left(p \cdot p^{\prime}+p \cdot p-p^{\prime} \cdot p^{\prime}-p^{\prime} \cdot p\right) \\
& +C\left(p \cdot p^{\prime}-p \cdot p-p^{\prime} \cdot p^{\prime}+p^{\prime} \cdot p\right) \\
= & A\left(p_{\mu}-p_{\mu}^{\prime}\right) \gamma^{\mu}+C\left(2 p \cdot p^{\prime}-2 p \cdot p\right)
\end{aligned}
$$

Sandwich Eq. 1.8 between $\bar{v}\left(p^{\prime}\right)$ and $v(p)$, then we notice the first term also vanishes as follows:

$$
\begin{aligned}
\bar{v}\left(p^{\prime}\right) q_{\mu} \Gamma^{\mu} v(p) & =A \bar{v}\left(p^{\prime}\right) p_{\mu} \gamma^{\mu} v(p)-A \bar{v}\left(p^{\prime}\right) p_{\mu}^{\prime} \gamma^{\mu} \bar{v}(p)+2 C \bar{v}\left(p^{\prime}\right) v(p) \cdot\left(p \cdot p^{\prime}-p \cdot p\right) \\
& =A \bar{v}\left(p^{\prime}\right) v(p) \cdot m-A \bar{v}\left(p^{\prime}\right) \bar{v}(p) \cdot m+2 C \bar{v}\left(p^{\prime}\right) v(p) \cdot\left(p \cdot p^{\prime}-p \cdot p\right) \\
& =2 C \bar{v}\left(p^{\prime}\right) v(p) \cdot\left(p \cdot p^{\prime}-p \cdot p\right)
\end{aligned}
$$

Thus $C$ must be zero, and based on the Gordon identity,

$$
\left.\bar{v}\left(p^{\prime}\right) \gamma^{\mu} v(p)=\overline{(} v\right)\left(p^{\prime}\right)\left[\frac{p^{\prime \mu}+p^{\mu}}{2 m}+\frac{i \sigma^{\mu \nu} q_{\nu}}{2 m}\right] v(p)
$$

$\Gamma_{\mu}$ is finally written as

$$
\Gamma^{\mu}\left(p^{\prime}, p\right)=\gamma^{\mu} F_{1}\left(q^{2}\right)+\frac{i \sigma^{\mu \nu} q_{\nu}}{2 M_{p}} F_{2}\left(q^{2}\right)
$$

where $M_{p}$ is the proton mass. $F_{1}$ and $F_{2}$ are functions of $q^{2}$ and are known as Dirac and Pauli form factors respectively. $F_{1}$ and $F_{2}$ are distinguished according to their helicity characteristics. $F_{1}$ represents the helicity-preserving part of the scattering while $F_{2}$ represents the helicity-flipping part. In the limit $q^{2}=0, F_{1}(0)=1$ and $F_{2}(0)=1.793=\kappa_{p}$ $=\mu_{p}-1$, in units of the nuclear magneton $\mu_{N}=e \hbar /\left(2 M_{p}\right) . \kappa_{p}$ is the proton anomalous 
magnetic moment and $\mu_{p}$ is the proton magnetic moment.

The differential cross section for $e-p$ scattering in the lab frame can be written as:

$$
\frac{d \sigma}{d \Omega}=\frac{2 \pi^{4}|M|^{2}}{4\left((k p)^{2}-m_{e}^{2} M_{p}^{2}\right)} \delta^{4}\left(k+p-k^{\prime}-p^{\prime}\right) \frac{d^{3} \vec{k}^{\prime}}{(2 \pi)^{3} 2 E^{\prime}} \frac{d^{3} \vec{p}^{\prime}}{(2 \pi)^{3} 2 E_{p}^{\prime}}
$$

where $M$ is the amplitude defined in Eq. 1.6. Integrating the $\delta$-function and expressing the proton current in terms of the Dirac and Pauli form factors, the differential cross section for an unpolarized beam and target can be written as:

$$
\frac{d \sigma}{d \Omega_{e}}=\left(\frac{d \sigma}{d \Omega}\right){ }_{\text {Mott }} \frac{E^{\prime}}{E}\left(F_{1}^{2}\left(Q^{2}\right)+\tau\left(F_{2}^{2}\left(Q^{2}\right)+2\left[F_{1}\left(Q^{2}\right)+F_{2}\left(Q^{2}\right)\right]^{2} \tan ^{2} \frac{\theta_{e}}{2}\right)\right)
$$

with $\tau=Q^{2} / 4 M_{p}^{2}$ and the Mott cross section is as follows:

$$
\left(\frac{d \sigma}{d \Omega}\right)_{\text {Mott }}=\frac{\alpha^{2} \cos ^{2} \frac{\theta_{e}}{2}}{4 E^{2} \sin \frac{\operatorname{se}_{e}}{2}}
$$

The Mott cross section is for the scattering of a spin- $1 / 2$ electron from a spinless, point-like target. The $E^{\prime} / E$ term in Eq. 1.13 is due to the recoil of the proton and is called the recoil factor. In terms of the electron's kinematics, this term can be written as:

$$
\frac{E^{\prime}}{E}=\frac{1}{1+\frac{2 E}{M_{p}} \sin ^{2}\left(\frac{\theta_{e}}{2}\right)}
$$

Another set of form factors, the Sachs form factors $G_{E p}$ and $G_{M p}$, are often introduced because the experimental cross section data are most easily analyzed in terms of them. The relations between the two sets of form factors are:

$$
\begin{gathered}
G_{E p}\left(Q^{2}\right)=F_{1}\left(Q^{2}\right)-\tau F_{2}\left(Q^{2}\right) \\
G_{M p}\left(Q^{2}\right)=F_{1}\left(Q^{2}\right)+F_{2}\left(Q^{2}\right)
\end{gathered}
$$


In the limit $Q^{2}=0$, Eq. 1.16 and Eq. 1.17 reduce to the normalization conditions respectively as:

$$
\begin{gathered}
G_{E p}(0)=F_{1}(0)=1 \\
G_{M p}(0)=F_{1}(0)+F_{2}(0)=\mu_{p}=2.793
\end{gathered}
$$

The two form factors, $G_{E p}$ and $G_{M p}$, carry information about the charge and current distributions of the proton and they are also called the electric and magnetic form factors respectively.

Substituting the $F_{1}\left(Q^{2}\right)$ and $F_{2}\left(Q^{2}\right)$ in Eq. 1.13 with $G_{E p}\left(Q^{2}\right)$ and $G_{M p}\left(Q^{2}\right)$, the elastic $e-p$ cross section can be written as:

$$
\frac{d \sigma}{d \Omega}=\left(\frac{d \sigma}{d \Omega}\right)_{\mathrm{Mott}}\left(\frac{E^{\prime}}{E}\right) \frac{1}{1+\tau}\left(G_{E p}^{2}\left(Q^{2}\right)+\tau\left[1+2(1+\tau) \tan ^{2} \frac{\theta_{e}}{2}\right] G_{M p}^{2}\left(Q^{2}\right)\right)
$$

where the cross terms between $G_{E p}\left(Q^{2}\right)$ and $G_{M p}\left(Q^{2}\right)$ are dropped. The virtual photon longitudinal polarization parameter $\epsilon$ is defined as:

$$
\epsilon=\left[1+2(1+\tau) \tan ^{2}\left(\frac{\theta_{e}}{2}\right)\right]^{-1}
$$

Thus the cross section can be written in a simpler form:

$$
\frac{d \sigma}{d \Omega}=\left(\frac{d \sigma}{d \Omega}\right)_{\text {Mott }}\left(\frac{E^{\prime}}{E}\right) \frac{1}{1+\tau}\left(G_{E p}^{2}\left(Q^{2}\right)+\frac{\tau}{\epsilon} G_{M p}^{2}\left(Q^{2}\right)\right)
$$

\subsection{Rosenbluth Separation Method}

A simplified form of the $e-p$ elastic cross section is given in Eq. 1.22. Then the reduced cross section is defined as follows, based to take advantage of its linear dependence on $\epsilon$ 
at the same $\tau$ :

$$
\left(\frac{d \sigma}{d \Omega}\right)_{\mathrm{R}}=\frac{\epsilon(1+\tau)}{\tau} \frac{E}{E^{\prime}}\left(\frac{d \sigma}{d \Omega}\right) /\left(\frac{d \sigma}{d \Omega}\right)_{\mathrm{Mott}}=G_{M}^{2}\left(Q^{2}\right)+\frac{\epsilon}{\tau} G_{E}^{2}\left(Q^{2}\right)
$$

Since the reduced cross section is linear in $\epsilon$ at a fixed $Q^{2}$, a linear fit of a range of reduced cross section values to $\epsilon$ gives $G_{E p}^{2} / \tau$ as the slope and $G_{M p}^{2}$ as the intercept. This is known as the Rosenbluth separation method and an example of its use is given in Fig. 1.3, where $G_{D}$ is the dipole form factor which is defined as follows:

$$
G_{D}=\frac{1}{\left(1+Q^{2} / \Lambda^{2}\right)^{2}}
$$

where $\Lambda^{2}=0.71 \mathrm{GeV}^{2}$

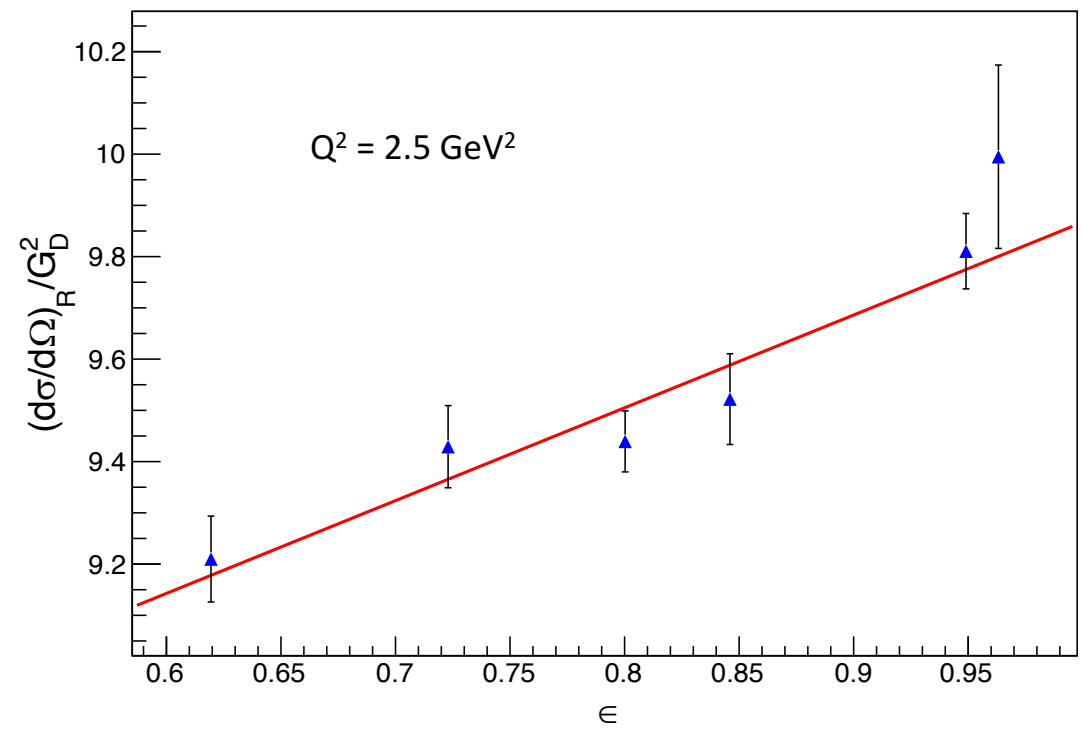

FIG. 1.3: Demonstration of the Rosenbluth separation method based on the data from [1]. $Q^{2}$ $=2.5 \mathrm{GeV}^{2}$.

The Rosenbluth separation method has been extensively used in the past several 
decades and has proved to be a very powerful method for investigating the proton's electric and magnetic form factors. However, there are limitations. Checking the cross section in terms of $G_{E p}$ and $G_{M p}$, we find that when $Q^{2}$ is small, the magnetic form factor $G_{M p}$ is suppressed and the cross section is dominated by the electric form factor $G_{E p}$ except when $\epsilon$ is close to 0 . Thus it is difficult to extract $G_{M p}$ with high accuracy at small $Q^{2}$ values. On the other hand, $G_{E p}$ is suppressed and the cross section is dominated by $G_{M p}$ when $Q^{2}$ is large. Thus it is difficult to extract $G_{E p}$ with high accuracy at large $Q^{2}$ values. Besides these limitations, the accuracies of the extracted $G_{E p}$ and $G_{M p}$ are also limited by the precision of the measured cross sections, which cover various kinematics to obtain different $\epsilon$ values at a fixed $Q^{2}$. The inconsistent acceptance, luminosity and detector efficiency at different kinematics can introduce systematic errors.

\subsection{Recoil Polarization Method}

Because of the limitations of the Rosenbluth separation method, physicists came up with another method to investigate the elastic form factors. When the polarization method is used, a longitudinally polarized beam of electrons is scattered off an unpolarized proton target. The polarization of the recoil proton is measured, which is transferred from the electron. In the single-photon exchange approximation of the ${ }^{1} \mathrm{H}\left(\vec{e}, e^{\prime} \vec{p}\right)$ reaction (shown in Fig. 1.4), there are only two non-zero polarization components, the transverse $P_{t}$ and the longitudinal $P_{l}$. The normal polarization component $P_{n}$ doesn't exist in elastic scattering in single-photon exchange.

The transverse and longitudinal components of the polarization, $P_{t}$ and $P_{l}$, can be 


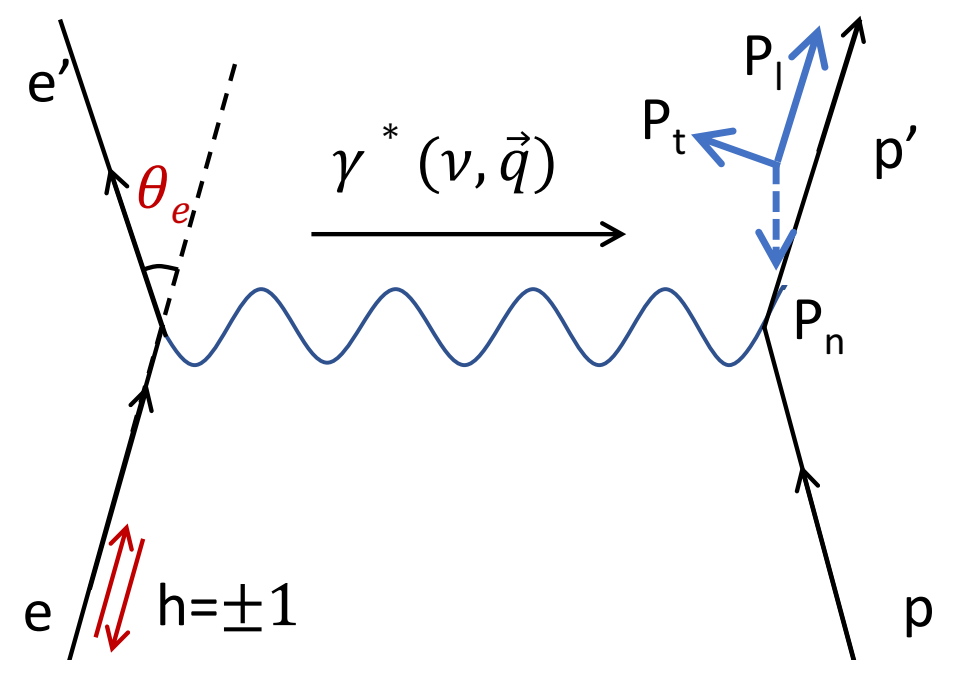

FIG. 1.4: Schematic diagram for polarization transfer from a longitudinally polarized electron to a proton with exchange of a virtual photon.

written in terms of $G_{E p}$ and $G_{M p}$ as follows $[6,7]$ :

$$
\begin{gathered}
I_{0} P_{l}=\frac{\left(E+E^{\prime}\right)}{M_{p}} \sqrt{\tau(1+\tau)} G_{M p}^{2}\left(Q^{2}\right) \tan ^{2}\left(\frac{\theta_{e}}{2}\right) \\
I_{0} P_{t}=-2 \sqrt{\tau(1+\tau)} G_{E p}\left(Q^{2}\right) G_{M p}\left(Q^{2}\right) \tan \left(\frac{\theta_{e}}{2}\right)
\end{gathered}
$$

The scattering angle is $\theta_{e}$ and $I_{o}$ is defined as:

$$
I_{o}=G_{E p}^{2}\left(Q^{2}\right)+\frac{\tau}{\epsilon} G_{M p}^{2}\left(Q^{2}\right)
$$

Eq. 1.25 and 1.26 show that the longitudinal and transverse polarization components are proportional to $G_{M p}^{2}$ and $G_{E p} G_{M p}$ respectively. Then dividing Eq. 1.25 by 1.26 , the ratio of $G_{E p} / G_{M p}$ is obtained:

$$
\frac{G_{E p}}{G_{M p}}=\frac{P_{t}}{P_{l}} \frac{E+E^{\prime}}{2 M_{p}} \tan \left(\frac{\theta_{e}}{2}\right)
$$

In polarization transfer experiments the transverse and longitudinal polarization com- 
ponents of the recoil proton are measured simultaneously, and the ratio of $G_{E p} / G_{M p}$ can be obtained directly. Compared with the Rosenbluth separation experiments, which measure the cross sections at various kinematics, the systematic errors of the polarization method are typically greatly reduced. Looking at Eq. 1.28, we notice that we don't need knowledge of the beam polarization or of the analyzing power of the polarimeter in order to extract the ratio of $G_{E p} / G_{M p}$, which makes the polarization method relatively easy to be achieved.

\subsection{Existing Measurements}

There has been much activity in the measurement of the proton electric and magnetic form factors for decades because of their fundamental importance. Fig. 1.5 shows a representative sample of the proton electric form factor $G_{E p}$ data obtained by the Rosenbluth separation method, where $G_{E p}$ is normalized to the dipole form factor $G_{D}\left(Q^{2}\right)$ (see Eq. 1.24). From the plot we can see that the proton electric form factor $G_{E p}$ is in excellent agreement with the dipole form factor, from very low $Q^{2}$ up to $1-2 Q^{2}$. As $Q^{2}$ goes higher, the uncertainty of the measured $G_{E p}$ increases rapidly, just as discussed in Chap. 1.4. The cross section becomes more and more dominated by the proton magnetic form factor $G_{M p}$ as $Q^{2}$ increases, and thus it becomes more and more difficult to extract $G_{E p}$ with high precision.

Fig. 1.6 shows a representative sample of the proton magnetic from factor $G_{M p}$ data obtained by the Rosenbluth separation method, where $G_{M p}$ is normalized to the dipole form factor. The data covers $Q^{2}$ up to $\sim 30 \mathrm{GeV}^{2}$. At the highest $Q^{2}$ point, the contribution to the cross section of the $G_{E p}$ related term is so small that the Rosenbluth separation method is no longer applicable and the assumption $G_{E p}=G_{M p} / \mu_{p}$ is used to extract $G_{M p}$ from a single cross section measurement. Even though we know this assumption is no 


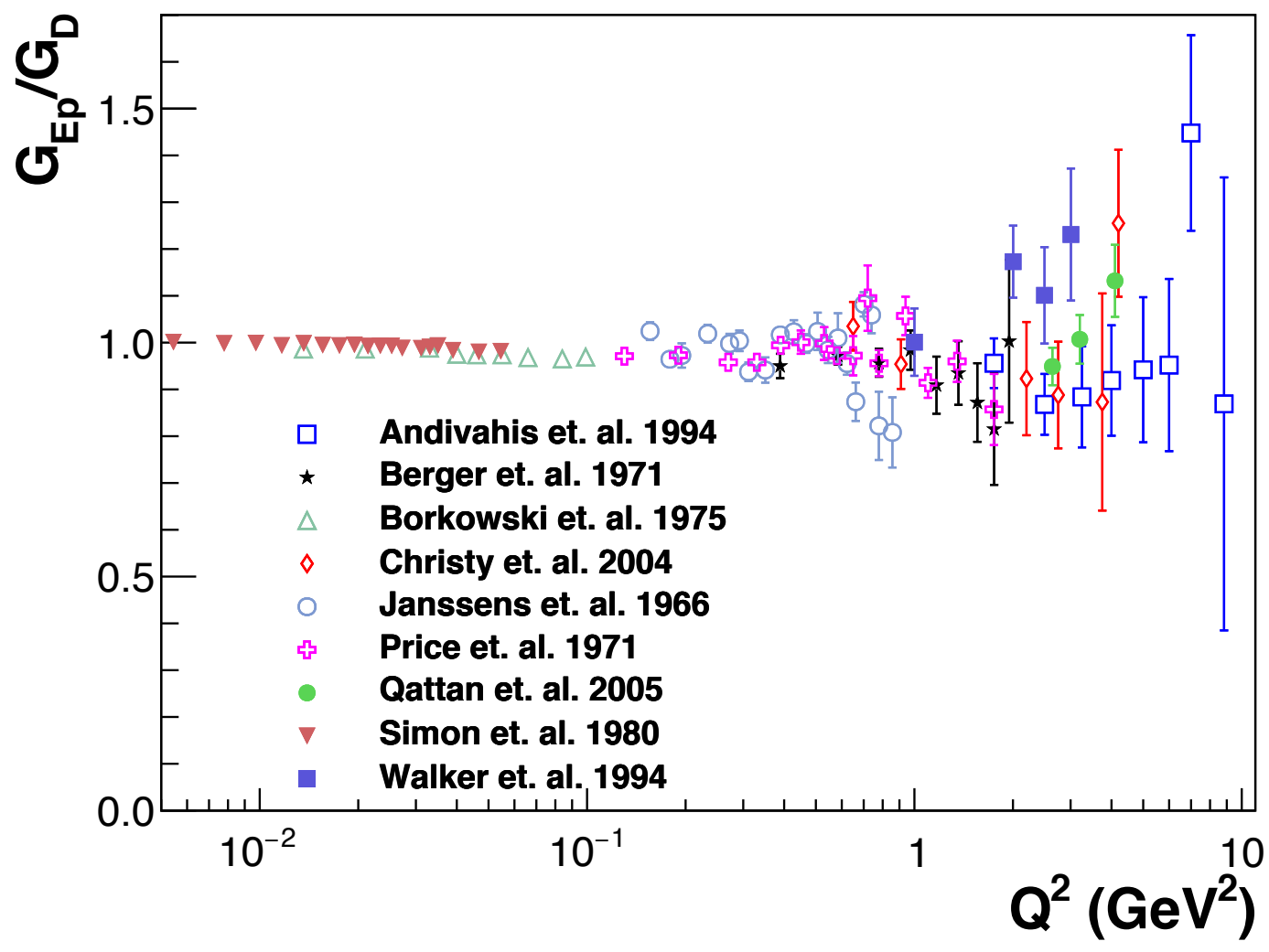

FIG. 1.5: Proton electric form factor $G_{E p}$ data obtained using the Rosenbluth separation method. Here $G_{E p}$ is normalized to the dipole form factor $G_{D}=\left(1+Q^{2} / \Lambda^{2}\right)^{-2}, \Lambda^{2}=$ $0.71 \mathrm{GeV}^{2}$. Data from references $[8,9,10,11,12,13,14,15,16]$. 
longer valid when $Q^{2}$ is above $1-2 \mathrm{GeV}^{2}$, the error introduced by the assumption is approximately as small as the electric form factor contribution, i.e., less than one percent. And at very low $Q^{2}$ the cross section is dominated by the electric term, thus $G_{M p}$ has large uncertainty at low $Q^{2}$ as shown in Fig. 1.6.

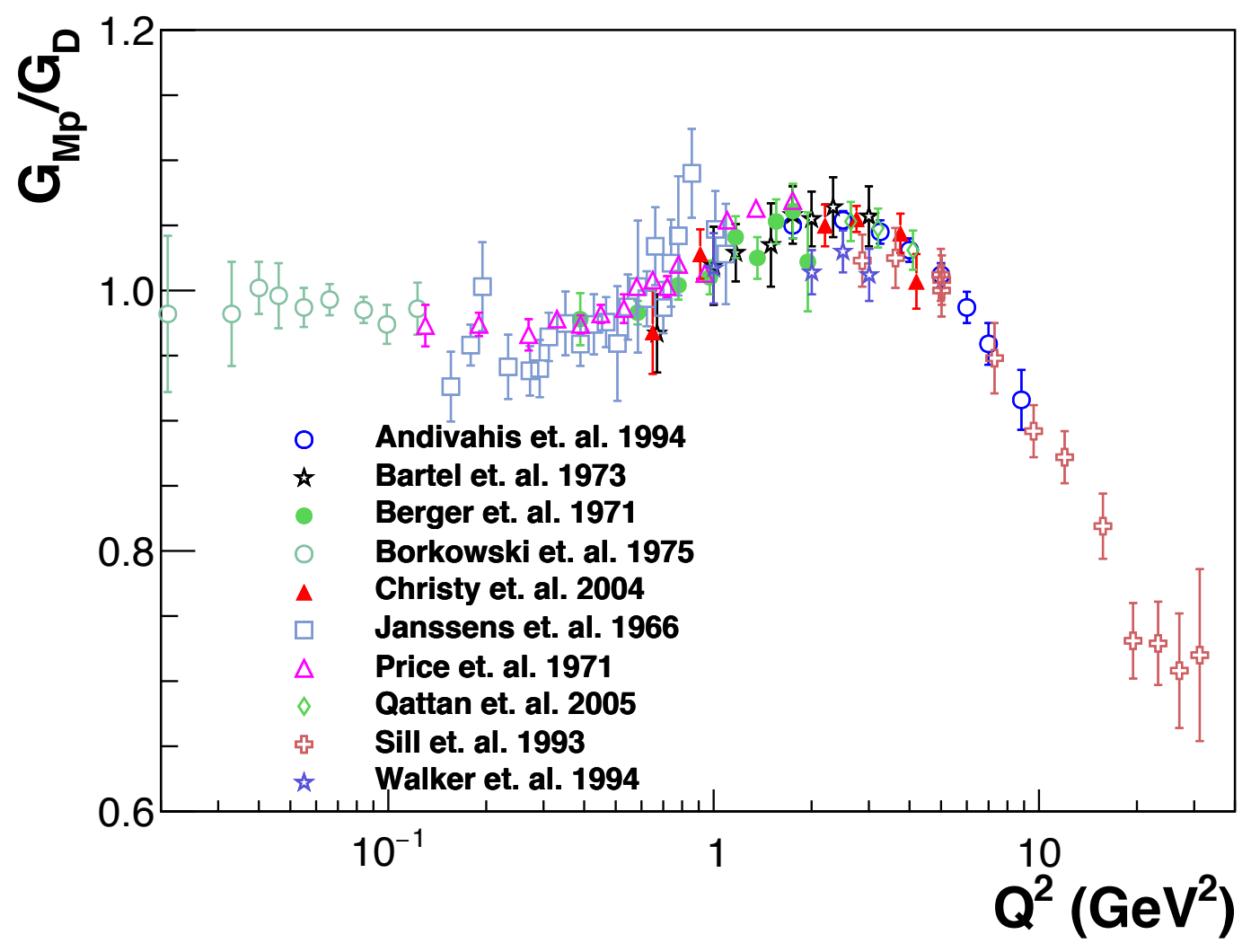

FIG. 1.6: Proton magnetic form factor $G_{M p}$ data obtained using the Rosenbluth separation method. Here $G_{M p}$ is normalized to the dipole form factor $G_{D}=\left(1+Q^{2} / \Lambda^{2}\right)^{-2}, \Lambda^{2}=$ $0.71 \mathrm{GeV}^{2}$. Data from references $[8,17,9,10,11,12,13,14,18,16]$.

The polarization technique was later developed to measure the proton electric and magnetic form factor ratio $\mu_{p} G_{E p} / G_{M p}$ and Fig. 1.7 shows a representative sample of the ratio obtained from both methods. In Fig. 1.7, the ratio obtained from these two different techniques are distinguished by their color: the blue points were obtained from the Rosenbluth separation method and the red points were obtained from the polarization technique. 
It is surprising that the polarization method results showed a dramatic deviation from the empirical scaling law $\mu_{p} G_{E p} / G_{M p}=1$ when $Q^{2}$ is larger than $2 \mathrm{GeV}^{2}$.

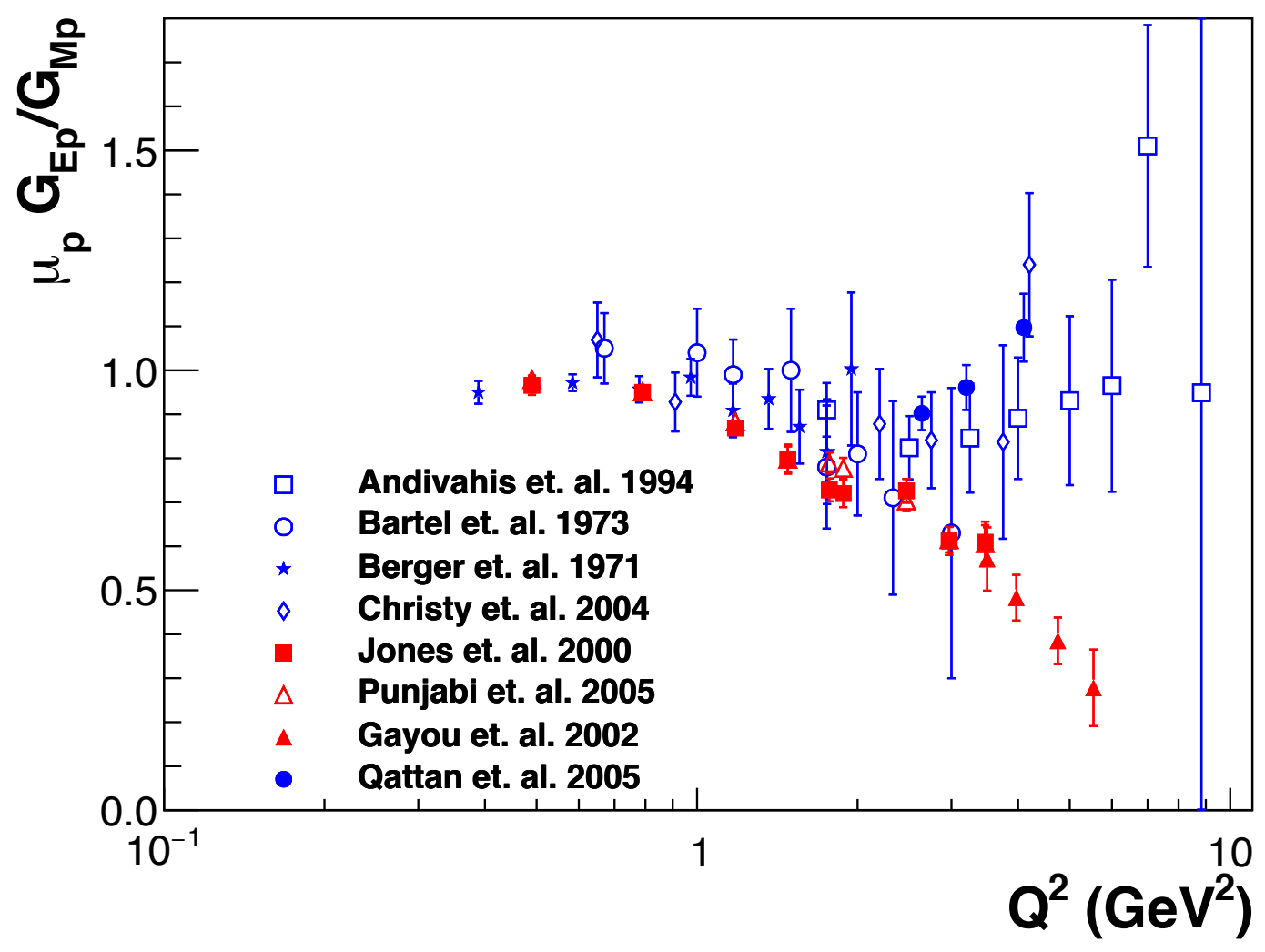

FIG. 1.7: Proton electric and magnetic form factor ratio $\mu_{p} G_{E p} / G_{M p}$ obtained with both the Rosenbluth separation and polarization techniques. The blue points were obtained from the Rosenbluth separation method and the red points were obtained from the polarization technique. Data from references $[8,17,9,11,19,20,21,14]$

An $\epsilon$-dependent modification of the cross section is necessary to explain the discrepancy between the form factor ratio obtained by the two methods. Recently a number of theoretical studies of two-photon-exchange (TPE) process $[22,23,24,25,26,27]$ have been performed because TPE is considered as the main source of this discrepancy. These studies show that TPE causes a strong angle-dependent correction to the elastic section and leads to large corrections to the extracted ratio. Fig. 1.8 shows the form factor ratio 
$\mu_{p} G_{E p} / G_{M p}$ obtained from the two methods and the comparison between the results before and after the TPE correction to the Rosenbluth separation data. Fig. 1.8 shows that the TPE correction greatly reduced the discrepancy and brings the Rosenbluth separation data much closer to and perhaps even in agreement with the polarization data. 

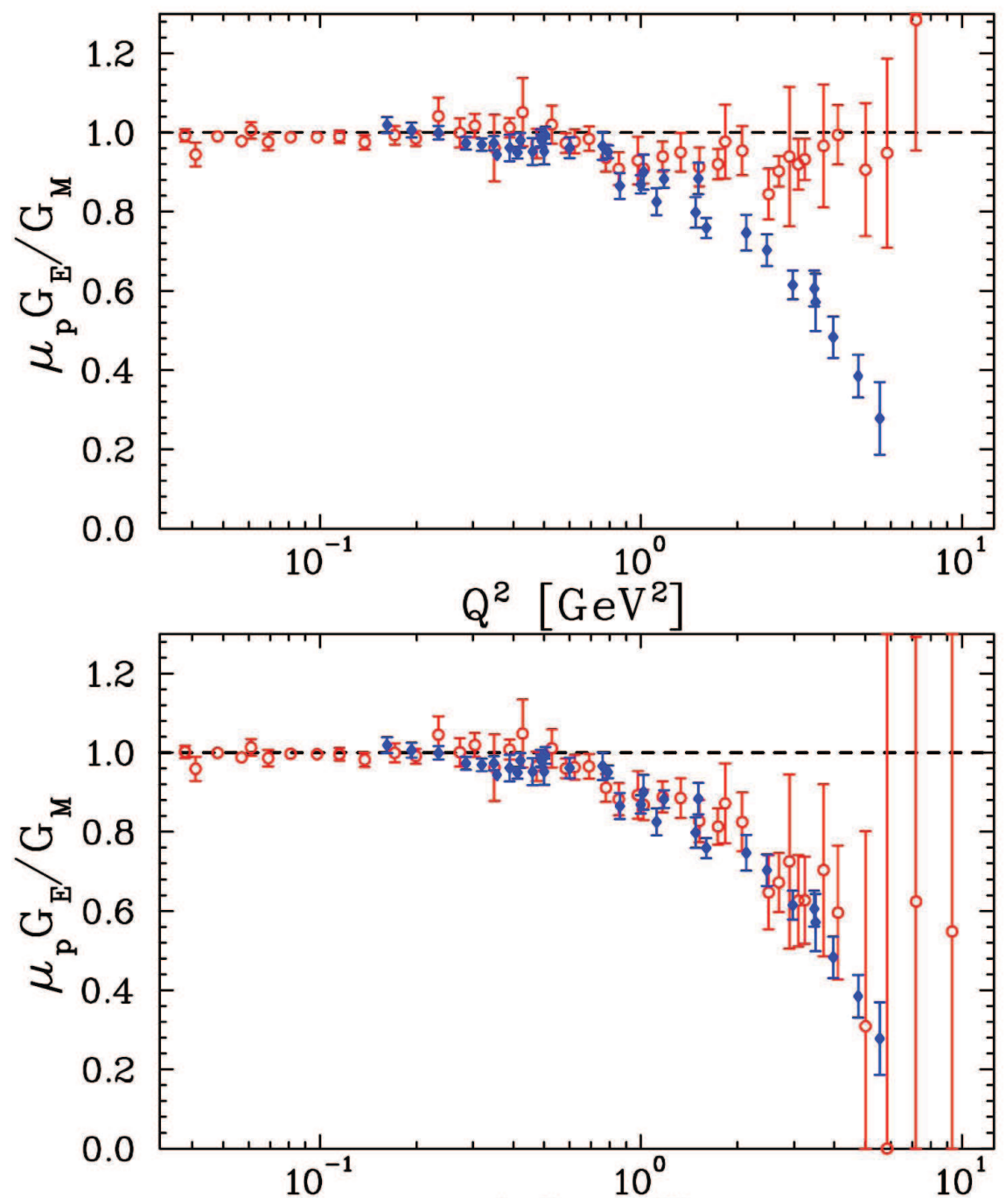

FIG. 1.8: Ratio $\mu_{p} G_{E p} / G_{M p}$ extracted from polarization transfer (filled diamonds) and Rosenbluth method (open circles). The top (bottom) figures show Rosenbluth method data without (with) TPE corrections applied to the cross sections. Figure is from Ref. [28]. 


\section{CHAPTER 2}

\section{Physics of Nucleon Form Factors}

\subsection{Vector Meson Dominance and Dispersion Rela- tions}

\subsubsection{Vector Meson Dominance}

Vector meson dominance (VMD) is one of the earliest models used to explain the global features of the nucleon form factors. Instead of a virtual photon exchange between the electron and the nucleon, the photon couples to the nucleon through vector mesons, $\rho(770)$, $\omega(782)$, and $\phi(1020)$, in the VMD model as shown in Fig. 2.1. The nucleon form factors are expressed in terms of photon-meson coupling strengths $C_{\gamma V}$ and meson-nucleon vector form factors $F_{j V}$ as follows:

$$
F_{j}^{i s, i v}\left(q^{2}\right)=\sum_{i} \frac{m_{i}^{2} C_{\gamma V i}}{m_{i}^{2}-q^{2}} F_{j V i}\left(q^{2}\right)=\sum_{i} \frac{m_{i}^{2} C_{\gamma V i}}{m_{i}^{2}+Q^{2}} F_{j V i}\left(q^{2}\right)
$$


where the sum is over vector mesons of mass $m_{i}$ and $i s$ and $i v$ correspond to the isoscalar and isovector electromagnetic currents respectively. The form factors are given by:

$$
2 F_{j p}=F_{j}^{i s}+F_{j}^{i v} \quad ; \quad 2 F_{j n}=F_{j}^{i s}-F_{j}^{i v}
$$

where $j=1,2$ and $p$ and $n$ denote the proton and neutron respectively.

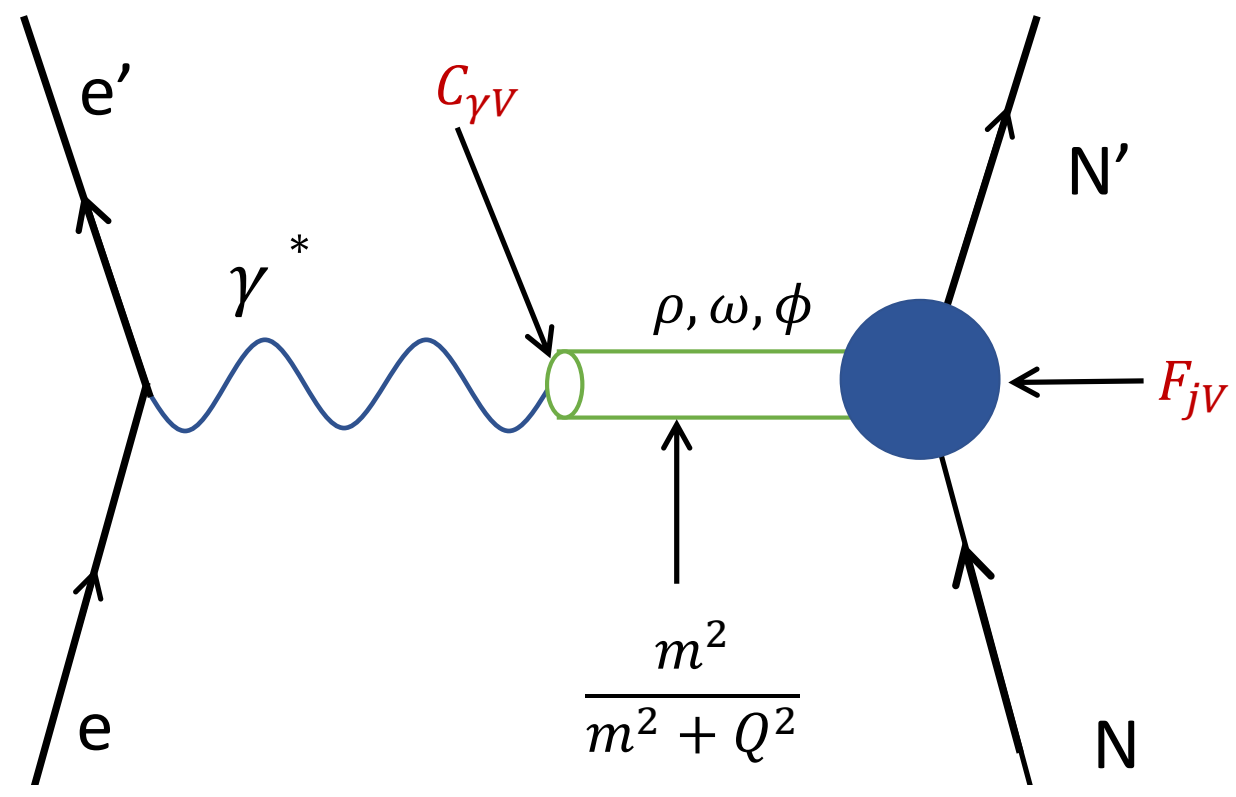

FIG. 2.1: Feynman diagram of the electron nucleon scattering in the VMD model The virtual photon couples to the nucleon through vector mesons, $C_{\gamma V}$ are the photon-meson coupling strengths and $F_{j V}$ are the meson-nucleon vector form factors.

The VMD model can explain the approximate dipole behavior of the nucleon form factors (see Eq. 1.24) by considering the contribution of two nearby vector meson poles with opposite residua. The two vector meson poles have masses $m_{V 1}$ and $m_{V 2}$ and if their residua are of equal magnitude but opposite sign, $a$ and $-a$ respectively, the form factors 
are proportional to:

$$
F_{1,2}\left(q^{2}\right) \sim \frac{a}{q^{2}-m_{V 1}^{2}}+\frac{-a}{q^{2}-m_{V 1}^{2}}=\frac{a\left(m_{V 1}^{2}-m_{V 2}^{2}\right)}{\left(q^{2}-m_{V 1}^{2}\right)\left(q^{2}-m_{V 1}^{2}\right)}=\frac{a\left(m_{V 1}^{2}-m_{V 2}^{2}\right)}{\left(Q^{2}+m_{V 1}^{2}\right)\left(Q^{2}+m_{V 1}^{2}\right)}
$$

In 1973, a semi-phenomenological fit was performed by Iachello et al. [29] based on the VMD model, to describe the proton form factors. The fit models the data for the

$\frac{G_{E p}}{G_{M p}}$ ratio well in the very low $Q^{2}$ regime and it predicted a linear decrease with $Q^{2}$. In 1985, Gari and Krümpelmann [30] extended the VMD model with additional functions in the definitions of $F_{1}$ and $F_{2}$ to include the perturbative QCD (pQCD) scaling relations at large $Q^{2}$. And later in the early 90's, the model was further extended [31] to include the contribution of the $\phi$ meson using an independent parametrization designed to conform to the constraints imposed on the $\phi N N$ coupling imposed by the OZI rule. In 2001, Lomon [32] extended the VMD fit by using $\rho(770), \omega(782), \phi(1020)$ and $\rho^{\prime}(1450)$ and fitting to new data. Fig. 2.2 shows the proton form factor ratio data from Jefferson Lab Hall A as a function of $Q^{2}$ with the several VMD model fits mentioned above.

\subsubsection{Dispersion Relations}

Assuming an unsubtracted dispersion relation (DR), the nucleon form factors $F\left(q^{2}\right)$ can be obtained as:

$$
F^{i s, i v}\left(q^{2}\right)=\frac{1}{\pi} \int_{t_{0}=4 m_{\pi}^{2}, 9 m_{\pi}^{2}}^{\infty} \frac{\operatorname{Im} F(t)}{t-q^{2}} d t
$$

where $F(t)$ generically stands for any of the four form factors and it is a spectral function. The integration is performed over the time-like region $\left(q^{2}>0\right)$ and thus $F(t)$ characterizes the electromagnetic structure of the nucleon in the time-like region. To extract the form factors in the space-like region $\left(q^{2}>0\right)$, a parameterization of $F(t)$ is required. For the isovector spectral function, a large non-resonant contribution starting from $t_{0}=4 m_{\pi}^{2}$ 


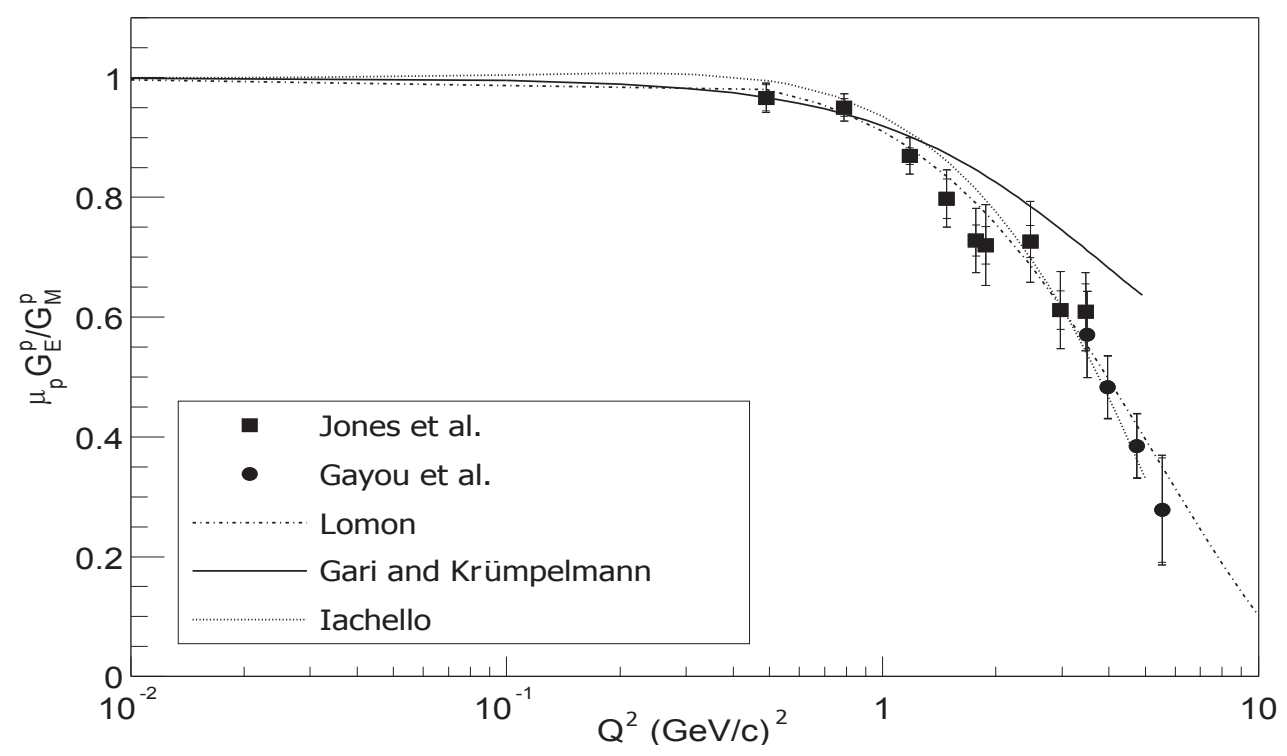

FIG. 2.2: The proton form factor ratio $\mu_{p} G_{E p} / G_{M p}$ data from Jefferson Lab Hall A with various VMD model fitting results. Figure is from Ref. [33].

and extending under the $\rho$-peak is seen. This is from the two-pion continuum. For the isoscalar spectral function, the integral starts at $t_{0}=9 m_{\pi}^{2}$, corresponding to $3 \pi$ intermediate states [5].

The VMD model is a special case of the dispersion analysis in which the spectral function is parametrized through the experimental data on the coupling of the nucleon and the electromagnetic current to all possible intermediate hadronic states contributing to the isoscalar and isovector dispersion integrals.

Höhler and collaborators [34] first estimated the two-pion continuum contribution. In the 1990s, the analysis was updated by Mergell, Meissner, and Drechsel [35] to include the nucleon time-like form factor data. A more recent dispersion analysis by Belushkin et al. [36] added the contributions from $\rho \pi$ and $K \bar{K}$. World data were analyzed in both the space-like and time-like regions and the fits are in general agreement with the data. Fig. 2.3 shows the nucleon space-like form factor fitting results compared to the world 
data.
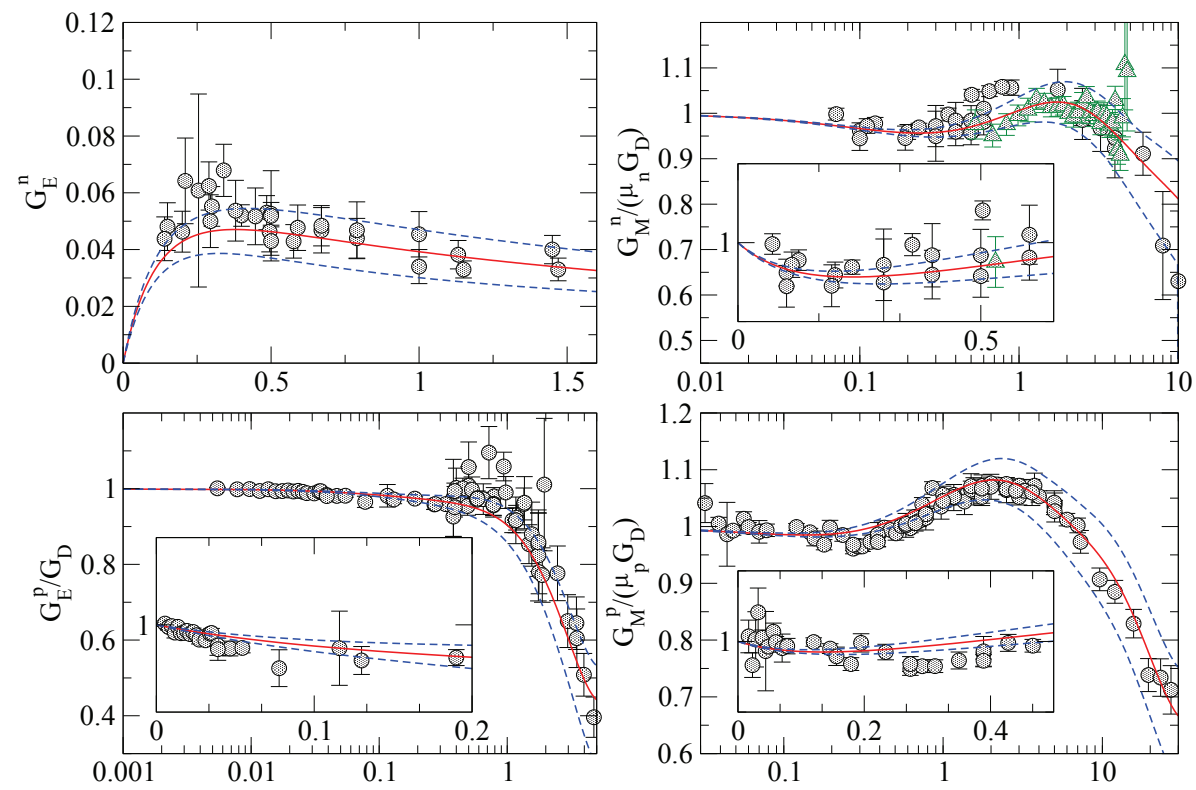

FIG. 2.3: Dispersion relation for the nucleon (space-like) form factors. The solid line gives the fit together with the world data (circles) including the JLab/CLAS data [37] for $G_{M n}$ (triangles), while the dashed lines indicate the error band. Figure is from Ref. [36]

\subsection{Constituent Quark Models}

In the constituent quark model $(\mathrm{CQM})$, the nucleon is treated as the ground state of a three quark system and the dominant effects of the gluonic degrees of freedom are absorbed into the mass of these three quarks and an effective potential. A famous example is the Isgur-Karl [38] model, in which the constituent quarks move in a long-range harmonic oscillator type confining potential, which is supplemented by a one-gluon-exchange quarkquark interaction. The one-gluon exchange leads to a color hyperfine interaction between quarks which accounts for a mass splitting between $N(939)$ and $\Delta(1232)$ and a mass splitting between the octet and decuplet baryons [39]. This model also predicts a small 
$D$-state probability for the nucleon ground state, which implies a non-spherical charge distribution.

The non-relativistic CQM, despite its simplicity, predicts the spectrum of low-lying baryons very well and gives a relatively good description of baryon static properties, such as the nucleon magnetic moments and the charge and magnetic radii. However, the quarks of QCD have small mass compared to the confinement mass scale and thus it is necessary to include the relativistic effects even at low momentum transfers when calculating the dynamic quantities such as the form factors. Also, when the momentum transfer is several times the nucleon mass, a relativistic description becomes crucial.

The relativistic constituent quark models (rCQM) are based on relativistic quantum mechanics and aim to formulate a mechanics where the Hamiltonian acts on a suitable Hilbert space, similar to the non-relativistic case. Dirac [40] explored the way to include relativity and distinguishes between three forms of dynamics, instant form, point form and light-front form, which differ in the choice of the kinematical subgroup of the Poincaré group. The Poincaré group contains ten generators, four space-time translations, three spatial rotations and three boosts.

In the instant form, the dynamical generators are the energy of the system and the three boost operators. The Einstein mass relation $p_{\mu} p^{\mu}=m^{2}$ takes the form as follows:

$$
p^{0}= \pm \sqrt{\vec{p}^{2}+m^{2}}
$$

There are two solutions for $p^{0}$ and thus it allows quark-antiquark pair creation and annihilation in the vacuum, which complicates the theory. On the other hand, rotations don't contain interactions and this makes it easy to construct states of definite angular momentum in the instant form.

In the point form, both boosts and rotations are kinematical and all four components 
of the four-vector operator are dynamical. It has the advantage that the angular momenta and Lorentz boosts are the same as the free case and the disadvantage that it complicates dealing with all four momentum components.

In the light-front form, seven of the dynamical variables are kinematical and the dynamical variables are the one component of the four momentum operator and two transverse rotations. This system has a simple Hamiltonian without negative energies, making it easy to separate the motion of the center of the mass of the system and the relative motion of the particles in the system. Diagrams involving quarks created out of, or annihilated into, the vacuum do not contribute because no negative energies are allowed. Its main drawback is that the construction of states with definite total angular momentum depends on the interactions among the quarks.

The light-front form calculation of the nucleon form factors was first introduced by Berestetsky and Terentev [47,48] and then developed by Chung and Coester [41]. This calculation starts from a rest frame nucleon wave function for the three-quark system which is fitted to the baryon spectrum and then a Melosh rotation [49] of each of the quark spinors is used to obtain the nucleon wave function in the light-front form. Fig. 2.4 shows this calculation is in surprisingly good agreement with the observed $G_{E p} / G_{M p}$, but it yields nucleon form factors dropping too fast at larger $Q^{2}$ when using constituent quark masses around $330 \mathrm{MeV}$. Schlumpf $[50,51]$ developed the nucleon light-front wave function from Chung and Coester, adopting a power law dependence in terms of the quark internal momentum variables. This model has only two free parameters, the constituent quark mass $m=263 \mathrm{MeV}$ and the confinement scale $\alpha=607 \mathrm{MeV}$, determined by fitting the magnetic moments and semileptonic decays of the baryon octet. Frank, Jennings and Miller $[42,52]$ used the wave function of Schlumpf and demonstrated that it leads to a violation of hadron helicity conservation, which leads to a falloff of $F_{2 p} / F_{1 p}$ slower than $1 / Q^{2}[53]$, in agreement with the $G_{E p} / G_{M p}$ polarization data. 


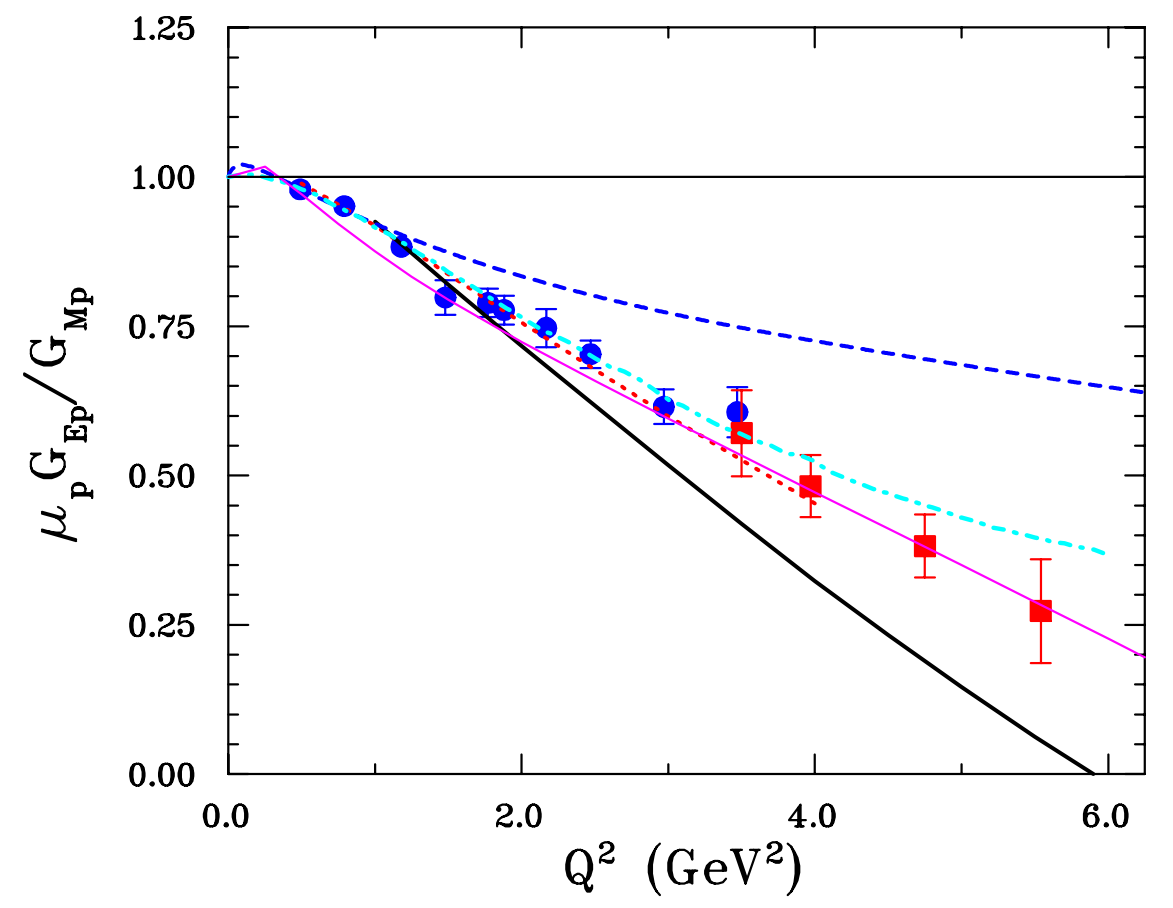

FIG. 2.4: Comparison of relativistic CQM calculations with data for $\mu_{p} G_{E p} / G_{M p}$. Dotted curve: front form calculation of Chung and Coester [41] with point-like constituent quarks; thick solid curve: front form calculation of Frank et al. [42]; dot-dashed curve : front form calculation of Cardarelli et al. [43, 44] with point-like constituent quarks; dashed curve: point form calculation of Boffi et al. [45] in the Goldstone boson exchange model with point-like constituent quarks; thin solid curve: covariant spectator model of Gross and Agbakpe [46]. The data are from Ref. [20] and Ref. [21]. Figure is from Ref. [5]. 
Cardarelli et al. $[43,44]$ have studied the consequences of the breaking of the $\mathrm{SU}(6)$ symmetry of the nucleon by the Melosh rotations using another approach to the relativistic constituent quark model in the light front form. The rest frame wave function was taken from the relativized Chapstick-Isgur model [54], which uses a one-gluon exchange potential. Recently Wagenbrunn, Boffi et al. calculated the nucleon form factors using the Goldstone-Boson-exchange (GBE) constituent quark model $[45,55,56]$. It was found when performing a point-form calculation of the nucleon form factors at larger $Q^{2}$ within the impulse approximation, a good overall description can be obtained, but $G_{M p}$ is underestimated and thus the ratio of $G_{E p} / G_{M p}$ is overestimated (shown as Fig. 2.4). The comparison between various CQM models and the data in the $Q^{2} 1$ to $6 \mathrm{GeV}^{2}$ region are shown in Fig. 2.4.

In conclusion, the constituent quark models include relativistic effects and have been successful in describing the nucleon form factors. However, they also have shortcomings as they do not satisfy all symmetry properties of the QCD Lagrangian.

\subsection{Perturbative (pQCD)}

In the theory of Quantum ChromoDynamics (QCD), the coupling constant $\alpha_{s}$ depends on $Q^{2}$ :

$$
\alpha_{s}\left(Q^{2}\right)=\frac{\alpha_{s}(0)}{1+\frac{\alpha_{s}(0)}{16 \pi^{2}}\left(11-\frac{2}{3} N_{f}\right) \ln \left(\frac{Q^{2}}{\Lambda_{Q C D}^{2}}\right)}
$$

where $N_{f}$ is the number of quark flavors and $\Lambda_{Q C D}$ is the QCD mass scale. The behavior of the strong coupling constant, which is high at low $Q^{2}$ and low at high $Q^{2}$, allows perturbative theory techniques to be applied when $Q^{2} \gg \Lambda_{Q C D}^{2}$.

In pQCD, the nucleon can be viewed as a system of three massless, weakly-interacting quarks moving collinearly in a reference frame in which the nucleon is moving with infinite 
momentum. Each quark carries a light-front 3-momentum fraction $x_{i,(i=1,2,3)}$, and the three quarks carry all the nucleon momentum, that is $x_{1}+x_{2}+x_{3}=1$. The transverse momenta of the quarks are assumed small enough to be neglected.

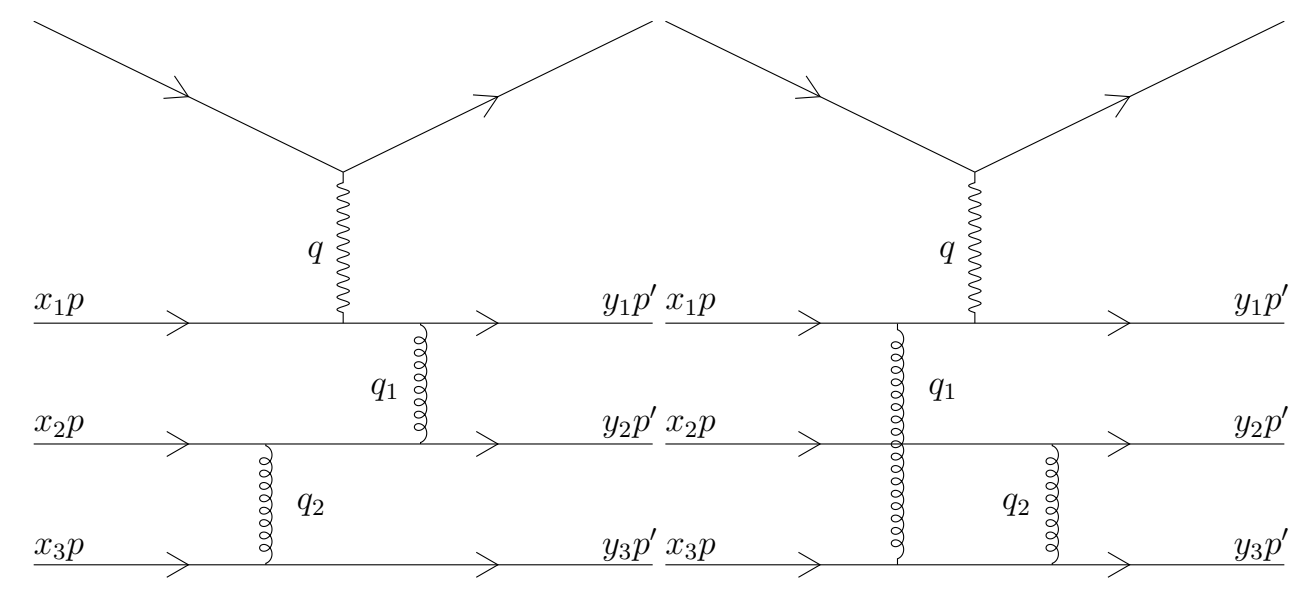

FIG. 2.5: Two typical Feynman diagrams contributing to $e N \rightarrow e N$ at leading order in $\alpha_{s}$ in $\mathrm{pQCD}$. The left and right panels show two different possibilities of the two required gluon exchanges. Figure is from Ref. [57].

For hard elastic scattering, the electron is scattered from a single quark and the quarks in the recoil nucleon redistribute their momentum so that they remain collinear after the scattering event. The struck quark interacts with the other quarks through gluon propagators and for $n$ quarks to remain collinear after the scattering, there must be at least $(n-1)$ gluons exchanged. Each possibility for $n=3$ is shown in Fig. 2.5. The nucleon form factor in this high $Q^{2}$ limit can be written in the factorized form:

$$
F\left(Q^{2}\right)=\int_{0}^{1} d x \int_{0}^{1} d y \Phi(y) T\left(x, y, Q^{2}\right) \Phi(x)
$$

where $d x \equiv d x_{1} d x_{2} d x_{3} \delta\left(1-x_{1}-x_{2}-x_{3}\right)$ and $d y \equiv d y_{1} d y_{2} d y_{3} \delta\left(1-y_{1}-y_{2}-y_{3}\right)$, with 
$x_{i,(i=1,2,3)}$ and $y_{i,(i=1,2,3)}$ being the momentum fraction of each quark of the initial and final nucleon, respectively. $T\left(x, y, Q^{2}\right)$ is the transition operator for the process $\gamma+3 q \rightarrow 3 q$ and $\Phi(x)$ and $\Phi(y)$ are the light-front quark distribution amplitudes (DAs) integrated over transverse momenta of quarks in the initial and final nucleons. The elasticity of the reaction is characterized by the fact that all quarks stay collinear in the process due to the struck quark transfering the absorbed momentum from the virtual photon to the other two quarks via gluons. Each gluon exchange contributes a factor of $\alpha_{s}\left(q_{i}^{2}\right)$ for the two $q q g$ vertices and each gluon propagator contributes a factor $1 / q_{i}^{2}$. The leading asymptotic $Q^{2}$ dependence of the form factor contained in $T$ can be guessed as:

$$
F\left(Q^{2} \rightarrow \infty\right) \propto \frac{\alpha_{s}\left(q_{1}^{2}\right) \alpha_{s}\left(q_{2}^{2}\right)}{q_{1}^{2} q_{2}^{2}} f(x, y) \propto \frac{1}{Q^{4}}
$$

The leading $Q^{-4}$ dependence applies to the helicity-conserving Dirac form factor $F_{1}$. For the helicity non-conserving Pauli form factor $F_{2}$, it is suppressed by a factor $m^{2} / Q^{2}$ relative to $F_{1}$, where $m$ is an effective quark mass. This leads to the leading $Q^{-6}$ dependence of $F_{2}$. The definition of the magnetic Sachs form factor implies the asymptotic behavior of $G_{M}$ must also be $G_{M}\left(Q^{2} \rightarrow \infty\right) \propto Q^{-4}$, according to the behavior of $F_{1}$ and $F_{2}$. The proton magnetic form factor has been measured to higher $Q^{2}$ values than any other nucleon form factor and Fig. 2.6 shows $Q^{4} G_{M p} / \mu_{p}$ extending to $Q^{2}$ above $30 \mathrm{GeV}^{2}$. The plots shows that $G_{M p}$ approximately satisfies the pQCD scaling prediction in the $Q^{2}$ range from 5 to $10 \mathrm{GeV}^{2}$, but $G_{M p}\left(Q^{2}\right)$ falls faster than the prediction at larger $Q^{2}$.

The assumption of quarks moving collinearly with the proton in pQCD has been investigated by Belitsky, Ji and Yuan [2]. They found that when including components in the nucleon light-cone wave functions with quark orbital angular momentum projection $l_{z}=1$, the behavior of $F_{2} / F_{1} \rightarrow \ln ^{2}\left(Q^{2} / \Lambda^{2}\right) / Q^{2}$ at large $Q^{2}$ is obtained with $\Lambda$ being a nonpertubative mass scale. Fig. 2.7 gives a test of this modified scaling prediction for $F_{2} / F_{1}$ 


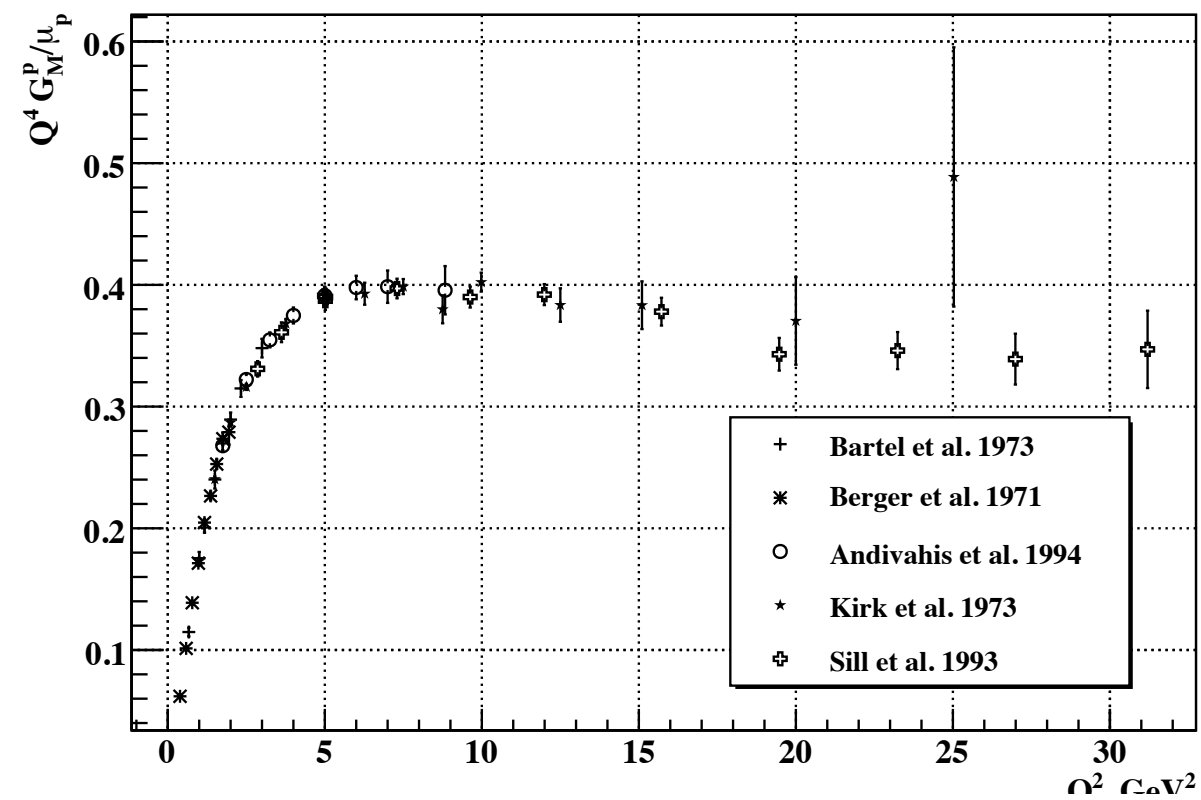

FIG. 2.6: $Q^{4} G_{M p} / \mu_{p}$ vs. $Q^{2}$. The data of Bartel et al. is from Ref. [17]. The data of Berger et al. is from Ref. [9]. The data of Andivahis et al. is from Ref. [8]. The data of Kirk et al. is from Ref. [58]. The data of Sill et al. is from Ref. [18]. Figure is from Ref. [57].

and it shows the prediction is in good agreement with the data starting at surprisingly low $Q^{2} . \Lambda$ is chosen as $0.3 \mathrm{GeV}$.

\subsection{Summary}

This chapter gives a brief discussion of various theoretical models of the nucleon form factors, however some important topics have been omitted. The lattice QCD simulations, the pion cloud mechanism, and the generalized parton distributions have not been covered. Readers can refer to Ref. [5], which has extensive discussion of these and additional topics. 


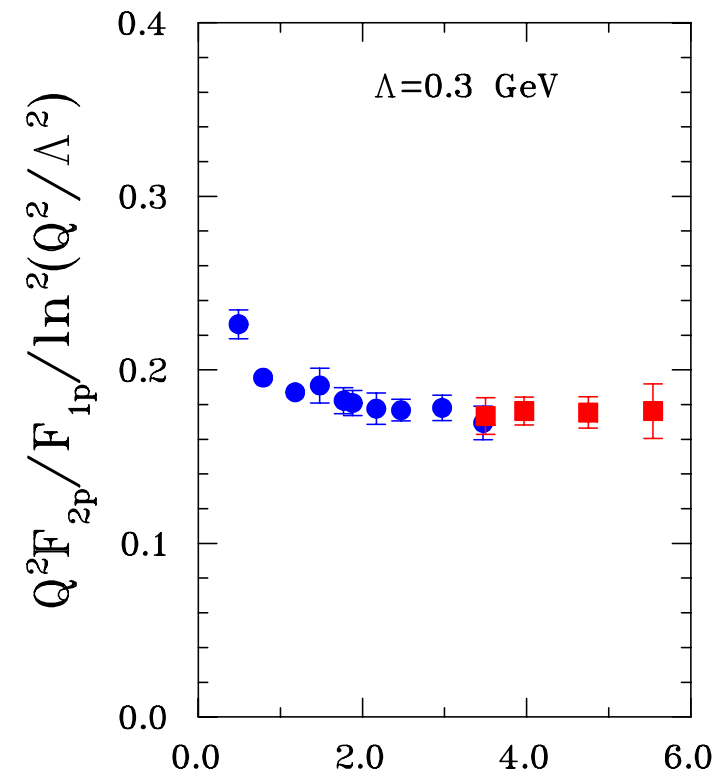

FIG. 2.7: Test of the modified scaling prediction for $F_{2 p} / F_{1 p}$ of Ref. [2]. Data of the blue solid circles are from Ref. [20] and data of the red solid squares are from Ref. [21]. Figure is from Ref. [5]. 


\section{CHAPTER 3}

\section{Experimental Setup}

\section{$3.1 \quad$ Overview}

Experiment E12-07-108 aimed to measure the elastic electron-proton cross-section with high precision in the $\mathrm{Q}^{2}$ range from $7 \mathrm{GeV}^{2}$ to $11 \mathrm{GeV}^{2}$. There were three production runs taken at the Thomas Jefferson National Accelerator Facility (JLab) in experimental Hall A (see Chap. 3.2.2) in the spring of 2015, the spring and fall of 2016. The blue diamonds in Fig. 3.1 show the $Q^{2}$ that were obtained by experiment E12-07-108. The $e-p$ elastic cross sections of four kinematics, with $Q^{2}=0.66,1.10,1.51$, and $1.65 \mathrm{GeV}^{2}$, were measured in the spring of 2015 and will be talked about in this thesis. When $Q^{2}>5 \mathrm{GeV}^{2}$, only a few previous measurements were performed with large uncertainties. To obtain better knowledge of the $e-p$ elastic cross sections at higher $Q^{2}$, experiment E12-07-108 took data in the $Q^{2}$ range 1.86 - $16.54 \mathrm{GeV}^{2}$ in the spring and fall of 2016 , which are being analyzed by experiment E12-07-108 collaboration and not discussed in this thesis.

A 3-D model of the experimental apparatus is shown in Fig. 3.2, which includes the beam-line elements (see Section 3.2), the liquid hydrogen target, left High Resolution Spec- 
trometer (Left-HRS) and right High Resolution Spectrometer (Right-HRS)(see Chapter 4).

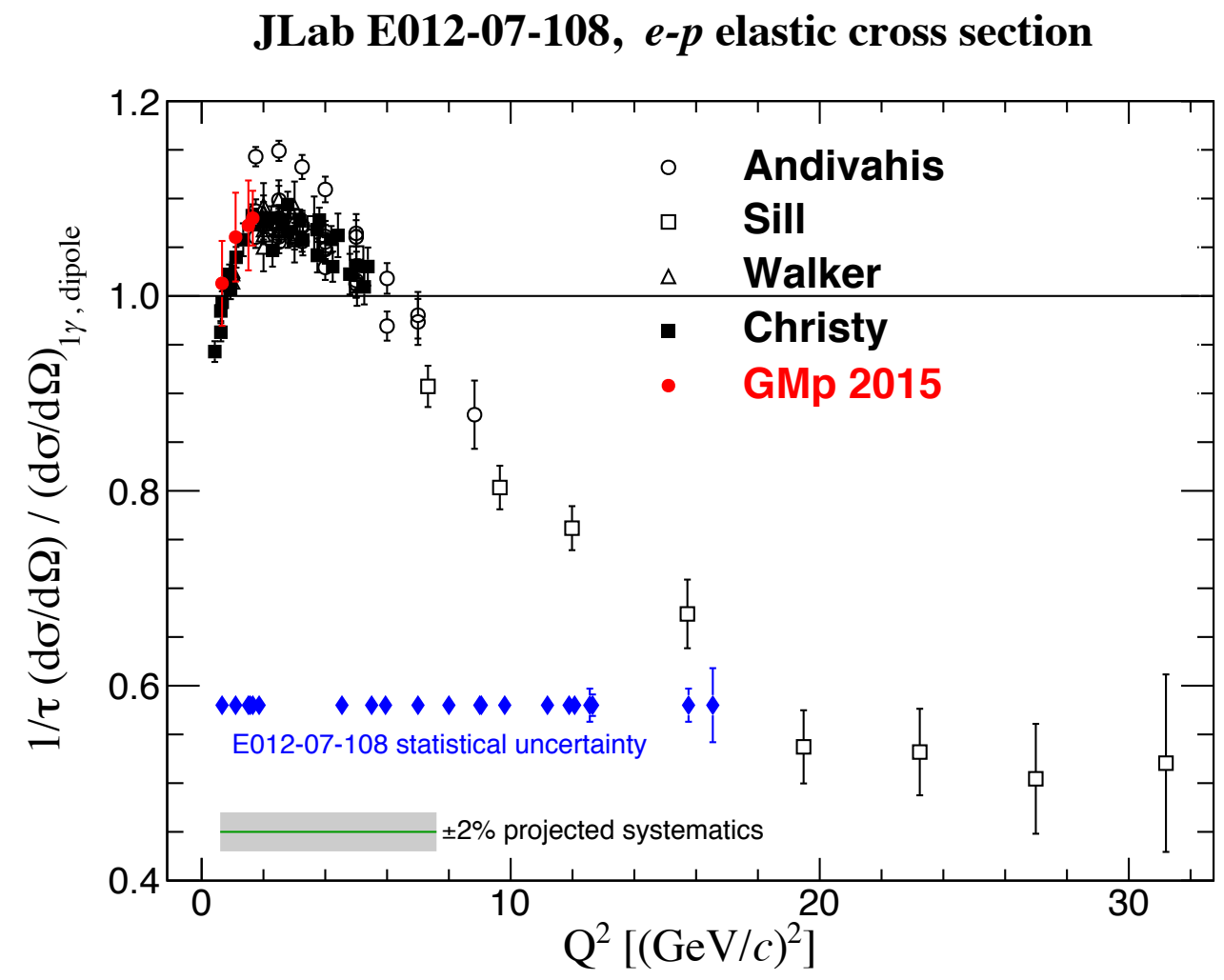

FIG. 3.1: The $Q^{2}$ measured by experiment E12-07-108 shown with the $e-p$ elastic cross sections obtained by the analysis in this thesis and previous measurements. The blue diamonds represent the $Q^{2}$ measured by experiment E12-07-108; the red circles show the $e-p$ elastic cross sections obtained the experimental data in the spring of 2015 (discussed in this thesis); black markers show the cross sections obtained from previous measurements; $(d \sigma / d \Omega)_{1 \gamma \text {,dipole }}$ is the reduced cross section obtained with $G_{E p}=G_{D}$ and $G_{M p}=\mu_{p} G_{D}$. Data from references $[8,18,16,11]$. 


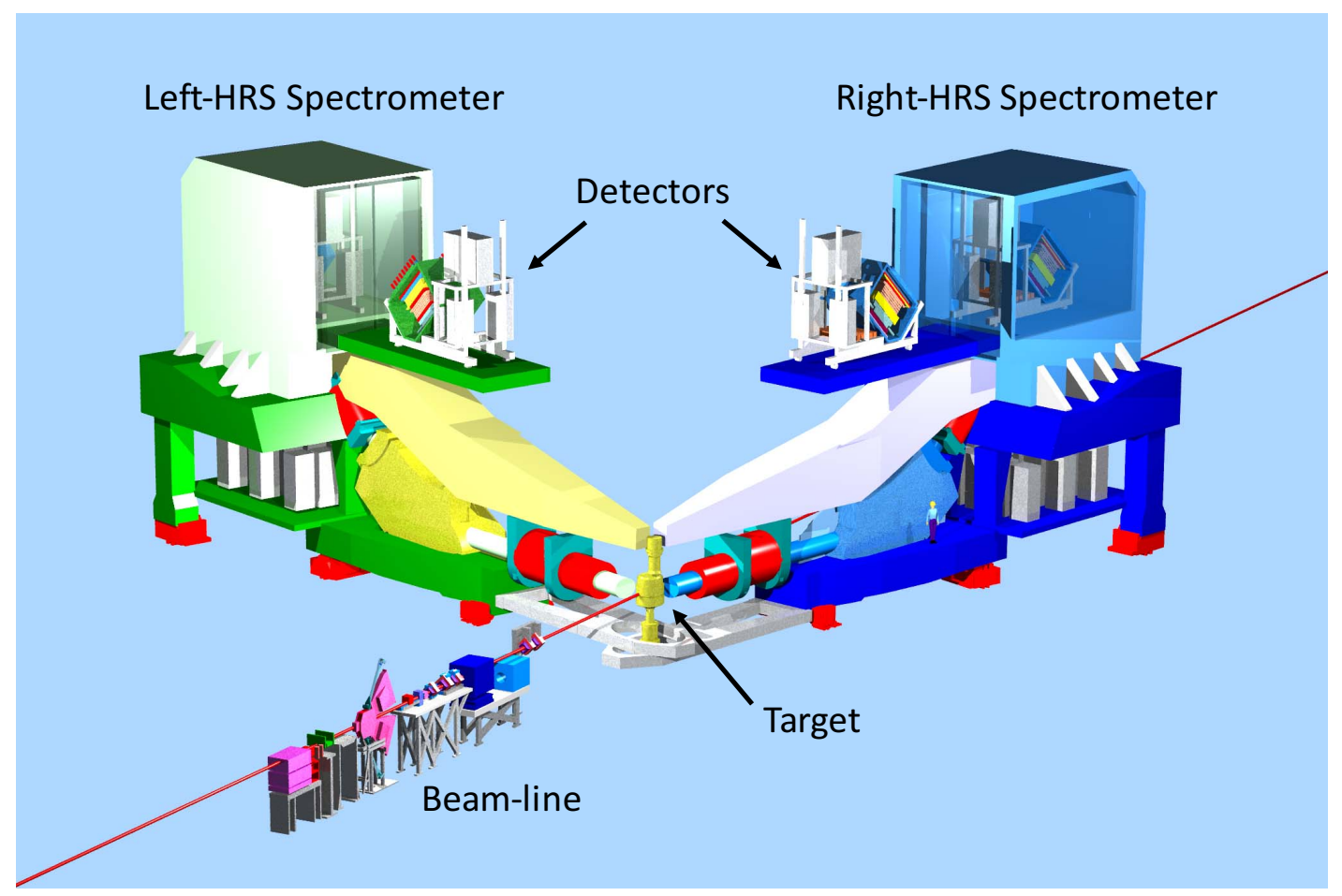

FIG. 3.2: 3-D model of the E12-07-108 experimental apparatus. The electron beam is incident on a liquid hydrogen target and the scattered electrons are detected by both the Left-HRS and Right-HRS spectrometers. The detectors are moved out the detector huts in this figure to get a better view. 


\subsection{Accelerator and Beam Line Components}

\subsubsection{Continuous Electron Beam Accelerator Facility}

Jefferson Laboratory's superconducting radiofrequency (srf) Continuous Electron Beam Accelerator Facility (CEBAF) provides multi-GeV continuous-wave (cw) electron beams for experiments at the nuclear and particle physics interface [59]. CEBAF was originally designed to accelerate electrons up to $4 \mathrm{GeV}$ by recirculating the beam up to four times through two superconducting linacs, each producing an energy gain of $400 \mathrm{MeV}$ per pass [60]. The original design goal was fully achieved in 1995 with an accelerating gradient of $5 \mathrm{MV} / \mathrm{m}$.

The srf technology makes it possible to upgrade the accelerator to higher energies. On August 6, 2000, Jefferson Lab announced that the CEBAF accelerator boosted the electron energy to $6.07 \mathrm{GeV}$ by raising the average cavity gradient to $7.5 \mathrm{MV} / \mathrm{m}$ and recirculating the beam up to five times. To expand the opportunity to study the quark structure of nuclei, JLab began the $12 \mathrm{GeV}$ construction in 2009. As shown in Fig. 3.3, using space already available in the accelerator tunnels, 10 higher performance cryomodules were installed.

As shown in Fig. 3.3, the main accelerator section of CEBAF consists of two antiparallel linacs connected by recirculating arcs. Each linac originally contained 20 cryomodules and 5 more were added for the $12 \mathrm{GeV}$ upgrade. The electrons are first injected into the accelerator from either a thermionic or a polarized gun and are accelerated to $50 \mathrm{MeV}$. Then the electrons are injected into the north linac and are accelerated through the crymodules. When the electrons reach the end of the north linac, they are steered through the $180^{\circ}$ bending arcs and are passed on to the south linac. The electrons are continuously accelerated in the south linac and when they reach the end, the electrons can be passed 


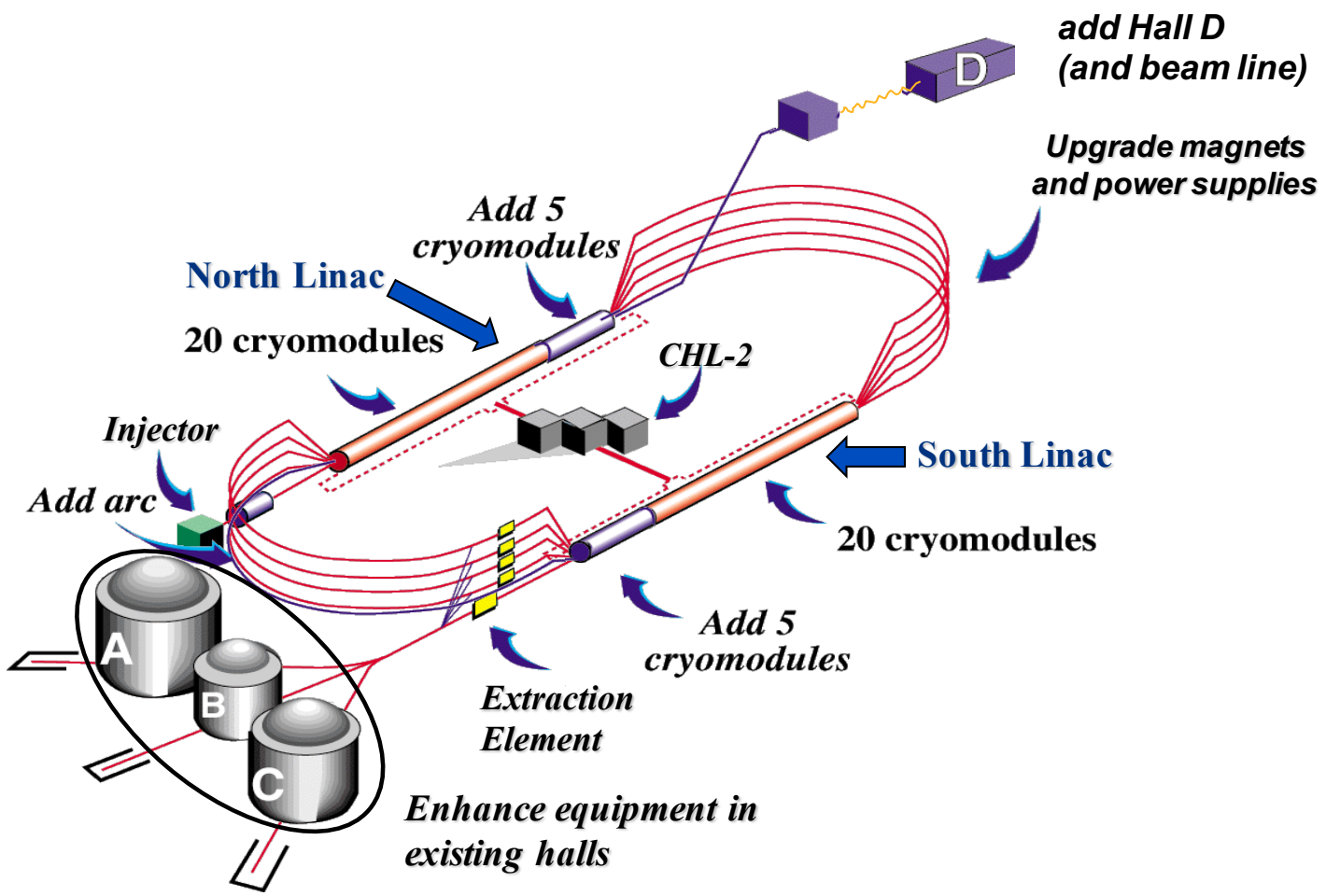

FIG. 3.3: Sketch of the CEBAF accelerator. The beam travels once through the north and south linacs with each recirculation, when it can be extracted into any of the Halls. 
on to the north linac or extracted to Hall A, B or C. When an experiment requires higher electron beam energy, the electrons can be recirculated up to five times. Different but correlated beams can be simultaneously delivered to four experiment halls.

\subsubsection{Hall A}

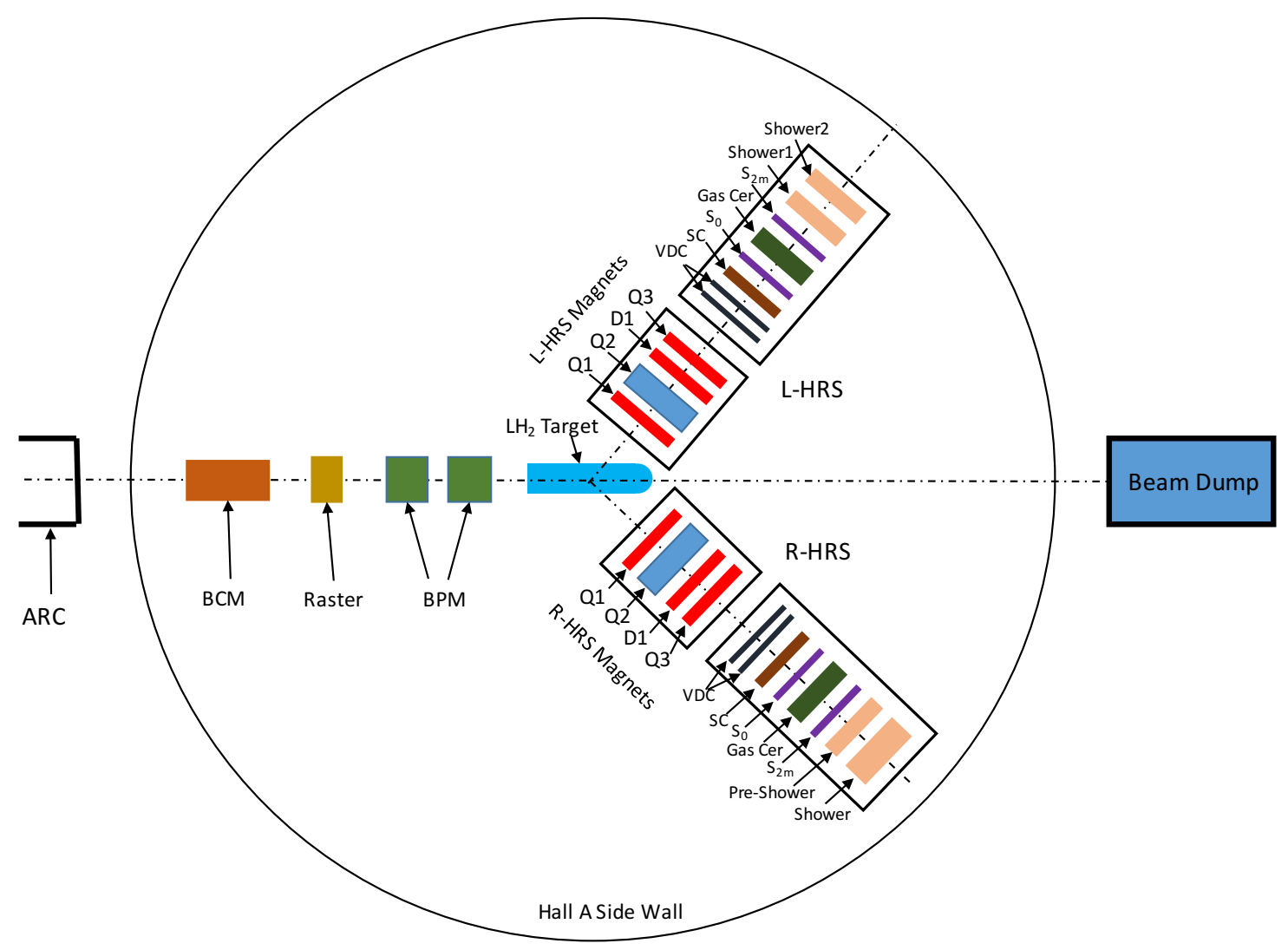

FIG. 3.4: Floor plan during experiment E12-07-108. VDC is the vertical drift chamber (see Chapter 4.2.1); SC is the straw chamber (see Chapter 4.2.2); $S_{0}$ and $S_{2 m}$ are the scintillators (see Chapter 4.2.3); Gas Cer is the gas Cherenkov detector (see Chapter 4.2.4); Pre-shower, shower, shower1, and shower2 are the electromagnetic calorimeters (see Chapter 4.2.5).

Experimental Hall A is one of the four experiment Halls at Jefferson Lab, with a diameter of $53 \mathrm{~m}$. The central components of Hall A are two identical high resolution spectrometers (HRSs), which provide a momentum resolution of better than $\Delta p / p=2$ 
$\times 10^{-4}$ and a horizontal angular resolution of better than $2 \mathrm{mrad}$ at a design maximum central momentum of $4 \mathrm{GeV}[60]$. The magnet configuration is of a QQDQ type with a vertical bend: a pair of superconducting quadrupoles followed by a $6.6 \mathrm{~m}$ long dipole and a third superconducting quadrupole.

The floor plan during experiment E12-07-108 is shown in Fig. 3.4. The key elements include the beam-line components, the target, the Left-HRS and the Right-HRS.

\subsubsection{Beam Energy Measurement}

\section{Arc Energy Method}

To measure the elastic $e$-p cross-section with high precision, the energy of the electron beam needs to be measured precisely. Experiment 12-07-108 uses the arc energy method [60] to determine the beam energy with an uncertainty of $\frac{\Delta E}{E}=5 \times 10^{-4}$ for the first pass beam.

When an electron travels in a uniform magnetic field, it moves in a circular trajectory, the radius of which depends on both the magnitude of the magnetic field and the momentum of the electron. Based on this principle, the arc energy method determines the energy by passing the electron in a known magnetic field and measuring the deflection of the beam in the arc section of the beam-line. The nominal bend angle of the beam in the arc section is $34.3^{\circ}$. The measurement is made when the beam is tuned in dispersive mode in the arc section. The momentum of the beam $p$ is related to the field integral of the eight dipoles $\left(\int \vec{B} \cdot \vec{d} l\right)$ and the net bend angle through the arc section $(\theta)$ by

$$
p=k \frac{\int \vec{B} \cdot \vec{d} l}{\theta}
$$

where $k=0.299792 \mathrm{GeV} \operatorname{rad} \mathrm{T}^{-1} \mathrm{~m}^{-1} / c$.

The arc setup is illustrated in Fig. 3.5. The arc energy method measures the magnetic 
field integral of the bending elements (eight dipoles in the arc) based on a reference magnet (9th dipole) and the actual bend angle of the arc using a set of wire scanners (SuperHarps). This method is an invasive measurement. It therefore cannot be made in parallel with the production data taking.

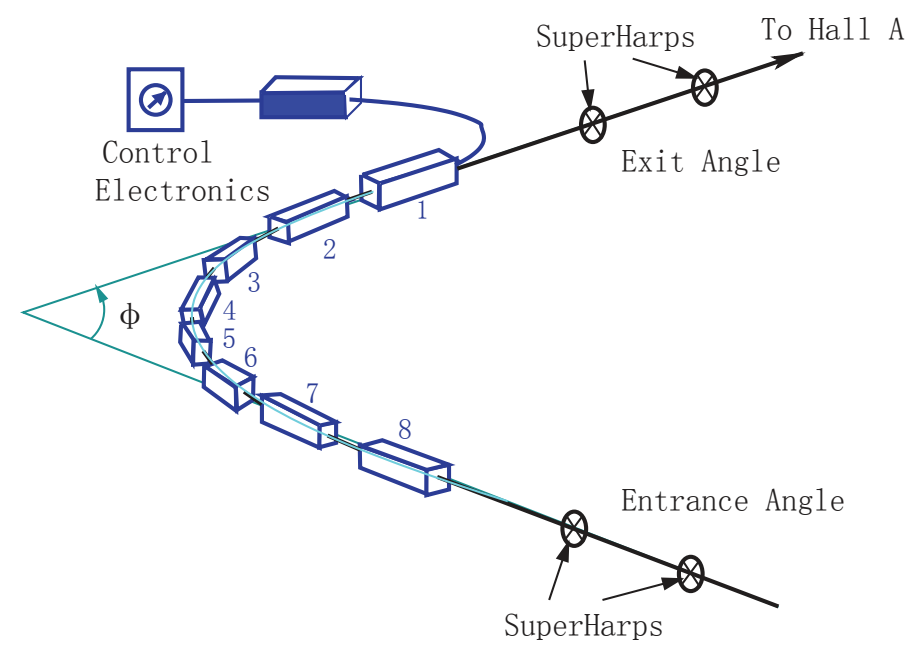

FIG. 3.5: Schematic of the arc energy measurement. Figure is from Ref. [61].

The beam energy during the experiment was monitored by the "Tiefenbach" value and cross checked by the arc measurement. This value is calculated by $\int \vec{B} \cdot \vec{d} l$ of Hall A arc and Hall A arc beam position monitors, giving the launch and exit angles of the beam through the arc. This number is continuously recorded in the data stream and was used in the data analysis. The relative error on the Tiefenbach energy is $\frac{\Delta E}{E} \leq 1 \times 10^{-3}$. The Arc measurements shows that the Tiefenbach energy needs to be scaled by 1.002 for the first pass beam and we use the Tiefenbach energy uncertainty as the beam uncertainty. 


\subsubsection{Beam Position and Direction}

Determining the position and direction of the beam at the target location is essential for experiment E12-07-108. Precise knowledge of the beam position and direction is important for spectrometer optics calibration and thus for event track-reconstruction.

Two Beam Position Monitors (BPMA and BMPB) are used to determine the position and direction of the beam at the target location [60]. They are located $7.345 \mathrm{~m}$ and 2.214 m upstream of the Hall A pivot. Each BPM is a resonant cavity with a 4-wire antenna with frequency tuned to match the RF frequency of the beam $(1497 \mathrm{MHz})$. The output signal of the BPM is proportional to the distance from the beam. The BPMs provide a non-destructive determination of the position and direction of the beam at the target location. The standard difference-over-sum technique is used to determine the relative position of the beam to $100 \mu \mathrm{m}$ for currents above $1 \mu \mathrm{A}[62,63]$. The absolute position of the beam can be determined from the BPMs by calibrating them with respect to wire scanners (Superharps), which are located adjacent to each BPM (see Fig. 3.6). The wire scanners are surveyed with respect to the Hall A coordinates at regular intervals and the results are reproducible at the level of $200 \mu \mathrm{m}$. The signal from each BPM is recorded by the DAQ for each event. The central beam position and direction at the target are linearly extrapolated using the beam position at both BPMs.

Experiment E12-07-108 used a liquid $\mathrm{H}_{2}$ target. To avoid overheating on a single spot of the target, the narrow ( $<0.3 \mathrm{~mm}$ wide) $\mathrm{CEBAF}$ beam was rastered to homogeneously cover a square shape with a size around $5 \mathrm{~mm}$ (vertical) $\times 3.6 \mathrm{~mm}$ (horizontal) during the spring of 2015 production run. The beam raster consisted of two sets of steering magnets, located about 23 meters upstream of the target. The shift of the beam relative to the average beam position was proportional to the current in the raster magnets, which was recorded by analog-to-digital converters for each event. 


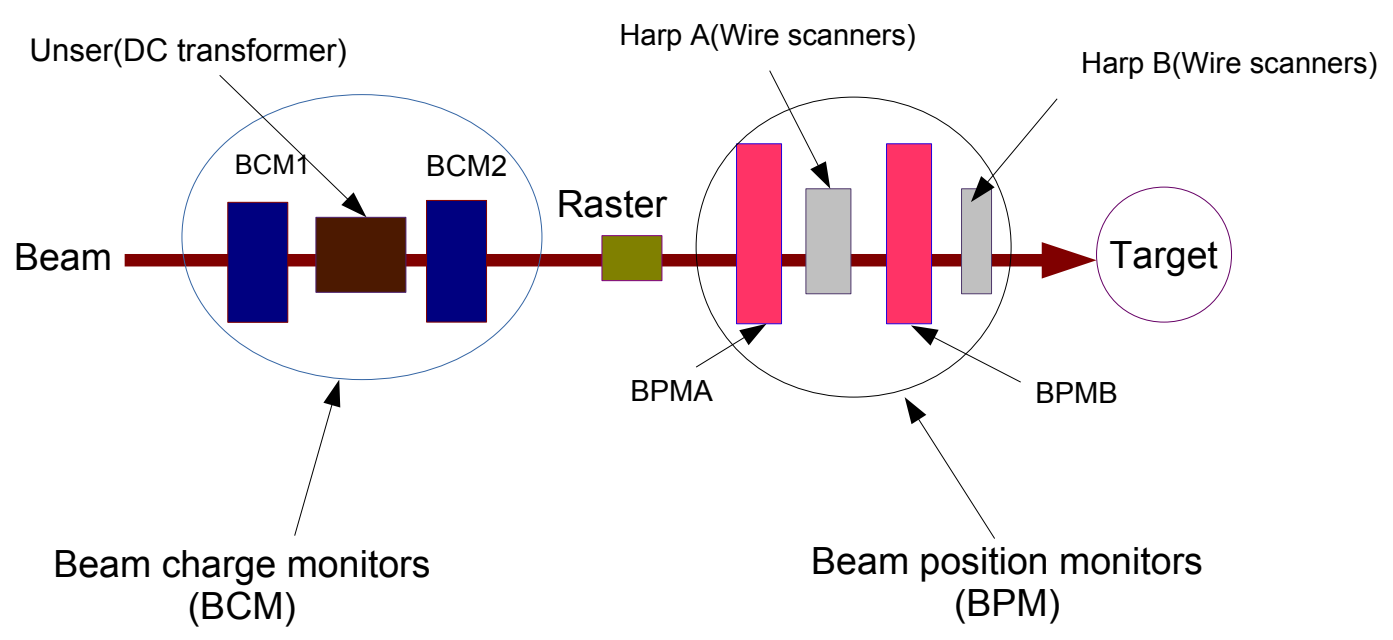

FIG. 3.6: Layout of Hall A beamline components for experiment E12-07-108.

\subsubsection{Beam Current Measurement}

The Beam Current Monitor (BCM) of Hall A is designed for a stable, low-noise, noninterfering beam current measurement. It consists of an Unser monitor, two RF cavities, associated electronics and a data-acquisition system [60]. As shown in Fig. 3.6, the Unser monitor is located between the two RF cavities, which are called BCM1 and BCM2. They are located $\sim 25 \mathrm{~m}$ upstream of the target and are enclosed in a temperature-stabilized magnetic shielding box. The Unser monitor is a Parametric Current Transformer which provides an absolute reference [64]. The monitor is calibrated by passing a known current through a wire inside the beam pipe and has a nominal output of $4 \mathrm{mV} / \mu \mathrm{A}$. As the Unser monitor's output signal drifts significantly on a time scale of several minutes, it cannot be used to continuously monitor the beam current and thus it is only used to periodically calibrate BCM1 and BCM2.

BCM1 and BCM2 are stainless steel cylindrical high-Q $(\sim 3000)$ waveguides which are tuned to the frequency of the beam $(1.497 \mathrm{GHz})$ resulting in voltage levels at their 
outputs which are proportional to the beam current. Each of the RF output signals from the two monitors is split into two parts (to be sampled or integrated).

\section{Sampled signals}

For the sampled BCM data, one of the amplifier outputs is sent to a high-precision digital AC voltmeter. This device provides, once every second, a digital output which represents the RMS of the input signal during that second. The resulting number is proportional to the beam charge accumulated during the corresponding second (or, equivalently, the average beam current for that second).

\section{Integrated signals}

For the integrated BCM data, the other amplifier output is sent to an RMS-to-DC converter, producing an analog DC voltage level. This level drives a Voltage-To-Frequency (VTOF) converter whose output frequency is proportional to the input DC voltage level. These signals are then fed to $200 \mathrm{MHz}$ VME scalers, which accumulate during the run. The scalers produce a number proportional to the time-integrated voltage level, and therefore more accurately represent the total beam charge. The regular RMS-to-DC output is linear for currents from about $5 \mathrm{~mA}$ to well above $200 \mathrm{~mA}$. A set of amplifiers has been introduced with gain factors of 1, 3 and 10. The higher-gain signals are used for lower current experiments, at the expense of saturation at high currents, while the gain factor 1 works best for the higher beam currents. Hence, there is a set of three signals coming from each of the upstream and downstream RF BCMs. These six signals are fed to scaler inputs of both Left-HRS and Right-HRS DAQs, providing redundant beam charge information. 
TABLE 3.1: List of the targets on the ladder of Hall A for experiment E12-07-108.

\begin{tabular}{c|c|c|c}
\hline \hline Target Name & Distance from Top (in) & Distance from Home (in) & Target Type \\
\hline Loop 1 & 0 & 25.558 & 4 cm Hydrogen \\
Loop 2 & 5.875 & 19.683 & 15 cm Hydrogen \\
Loop 3 & 11.75 & 13.808 & 15 cm Hydrogen \\
4 cm Dummy & 14.963 & 10.595 & Aluminum 3003 \\
15 cm dummy & 16.163 & 9.395 & Aluminum 7075 \\
optics & 16.713 & 8.845 & Carbon Graphite \\
empty 1 & 17.713 & 7.845 & N/A \\
empty 2 & 18.263 & 7.295 & N/A \\
BeO & 18.813 & 6.745 & Beryllium Oxide \\
Carbon hole & 19.363 & 6.195 & Carbon Graphite \\
Carbon & 19.913 & 5.645 & Carbon Graphite \\
Home & - & 0 & N/A \\
\hline
\end{tabular}

\subsubsection{Hall A Cryogenic Target System}

The cryogenic target system of Hall A is mounted on a ladder inside the vacuum scattering chamber. A target is chosen to intersect with the beam by moving the ladder vertically up and down. This operation is done remotely in the control room (counting house).

The cryogenic target has three independent loops. During spring 2015, Loop 1 (4 cm target) and Loop 2 (15 cm target) were filled with liquid hydrogen $\left(\mathrm{LH}_{2}\right)$ and Loop 3 (15 cm target) was filled with deuterium gas. The loops have cylindrical aluminum cells ending with a hemispherical tip. The Loop2 target was used during spring 2015 at a constant temperature of $19 \mathrm{~K}$ and pressure of 25 psi with a density of $0.0732 \mathrm{~g} / \mathrm{ml}$. The length of the target is $15 \mathrm{~cm}$, the width of the target is $7.62 \mathrm{~cm}$ and the radius of the tip is $3.81 \mathrm{~cm}$. The sidewalls of the aluminum cell are $0.173 \mathrm{~mm}$ thick with an entrance (upstream) window of $0.175 \mathrm{~mm}$ thick and an exit (downstream) exit window of 0.132 mm thick. The ladder also includes one $4 \mathrm{~cm}$ and one $15 \mathrm{~cm}$ dummy target for targetwindows background measurement, an optics target (multi-foil carbon) for event trajectory 


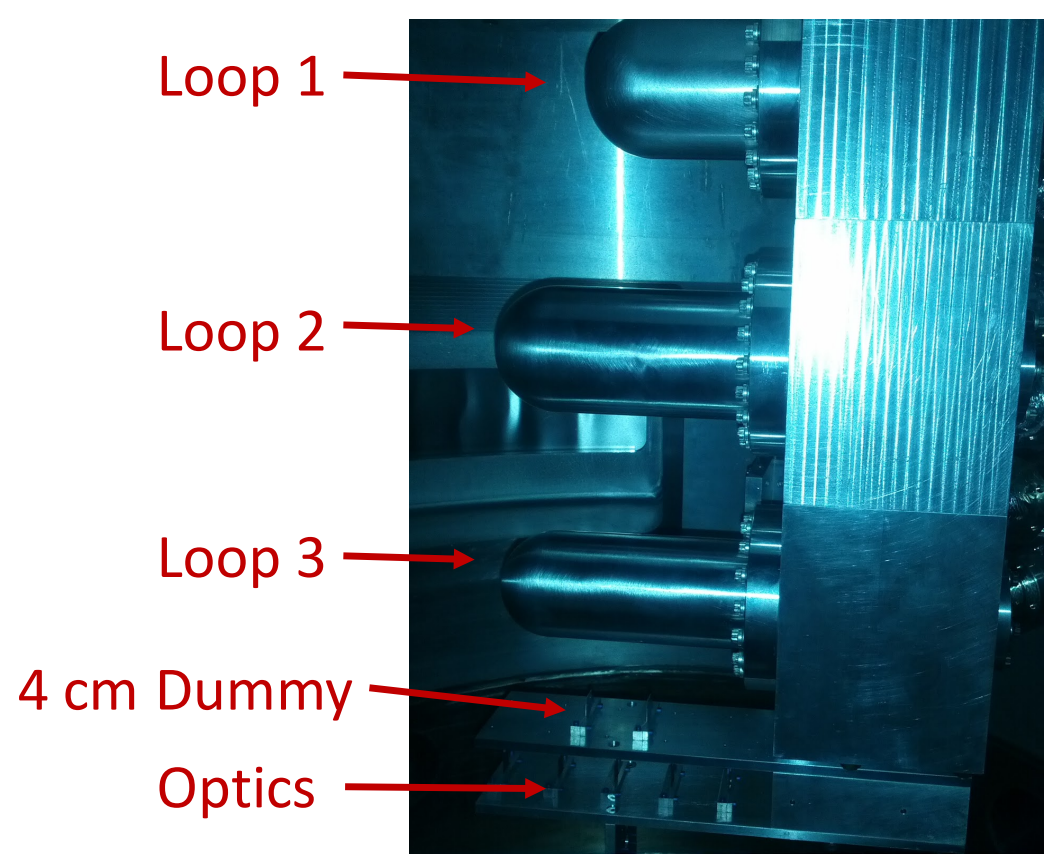

FIG. 3.7: The photograph of the target ladder inside the scattering chamber in the spring of 2015 .

reconstruction calibration and a carbon hole target for beam position calibration. All of the targets on the ladder and their positions are listed in Table 3.1. The dummy targets are aluminum foils mounted on separate frames with foils located at $z$ positions corresponding to the cryogenic target exit and entrance windows. The optics target consists of 5 carbon $(99.95 \%$ C) foils in a linear array with foils separated by $3.75 \mathrm{~cm}$. A photograph (see Fig. 3.7) shows what the target looks like in the vacuum scattering chamber. 


\section{CHAPTER 4}

\section{High Resolution Spectrometers}

\section{(HRSs)}

\subsection{Overview}

The High Resolution Spectrometers (Left-HRS and Right-HRS) are the standard experimental apparatus in Hall A [60]. As shown in Fig. 4.1, the vertical bending design includes a pair of superconducting $\cos (2 \theta)$ quadrupoles followed by a $6.6 \mathrm{~m}$ long dipole magnet with focusing entrance and exit pole faces and includes additional focusing from a field gradient in the dipole. Following the dipole is a third superconducting $\cos (2 \theta)$ quadrupole. The first quadrupole, Q1, is convergent in the dispersive (vertical) plane. The second and third quadrupoles of each spectrometer, Q2 and Q3, are identical in design and construction because they have similar field and size requirements and they both provide transverse focusing. 


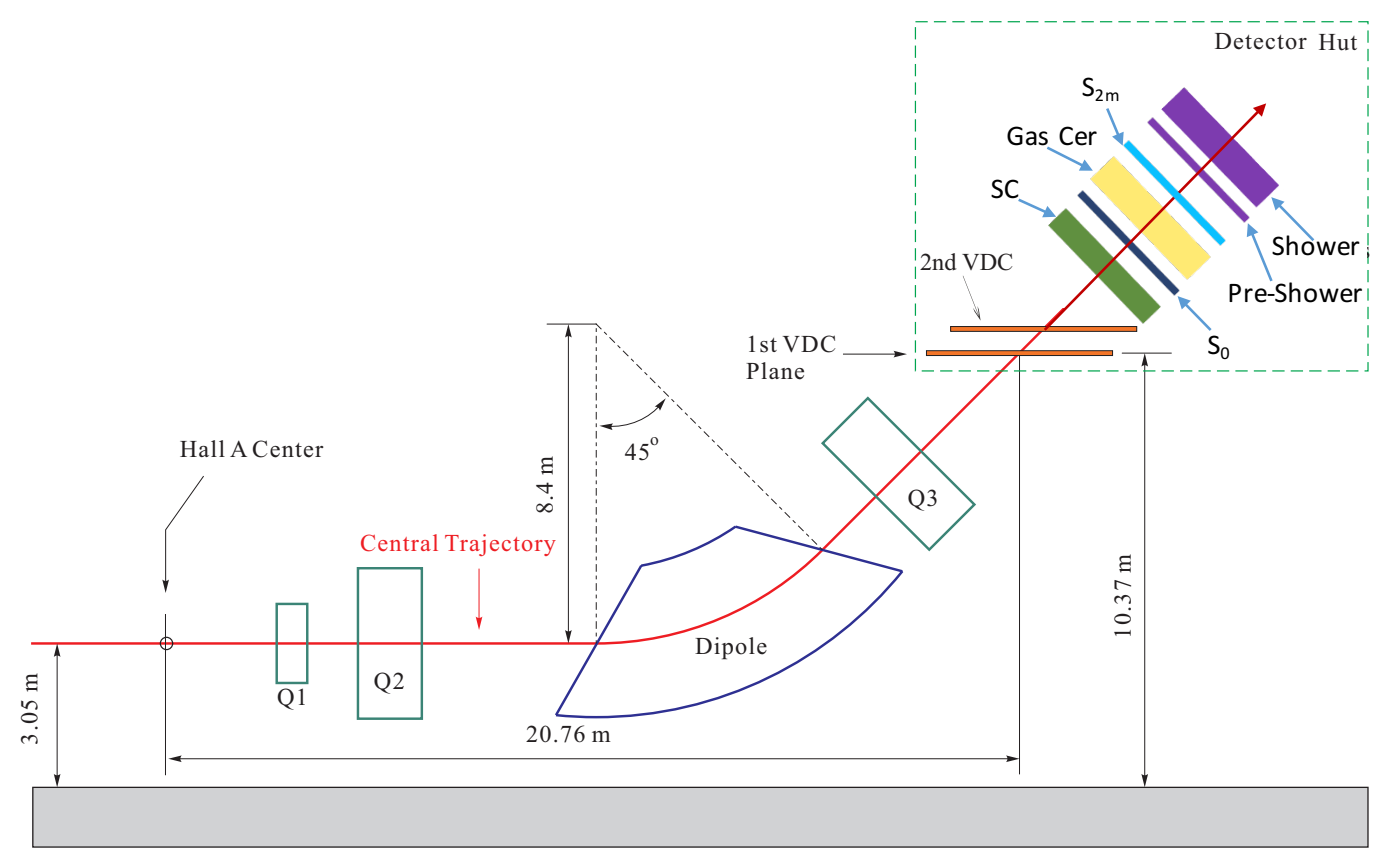

FIG. 4.1: Schematic of Hall A High Resolution Spectrometer and the detector package for the E12-07-108 experiment. 


\subsection{Detector Packages}

As shown in Fig. 4.1, the detectors and all of the Data-Acquisition (DAQ) electronics are located inside a detector hut which protects them from background radiation.

\subsubsection{Vertical Drift Chambers (VDCs)}

The tracking information was provided by a pair of vertical drift chambers (VDCs) on each arm of the HRSs [65], which were the first devices in the detector hut as shown in Fig. 4.1. In the dual-VDC system (see Fig. 4.2), the lower VDC is positioned to coincide as closely as possible with the spectrometer focal plane, and a second VDC is located above it to enable precise angular reconstruction of particle trajectories. The two VDC chambers are placed horizontally with the long edge along the dispersive direction, and with a vertical

separation of about $335 \mathrm{~mm}$. In each chamber, there are two wire planes, orthogonal to each other which lie in the horizontal plane of the laboratory, and both are oriented at $45^{\circ}$ with respect to the dispersive direction.

To make the electric field in the drift region more uniform and to reduce cross-talk between adjacent drift cells, drift chambers commonly use guard wires. They are generally located in the plane of, and in between, the sense wires, and are held at ground potential $[66,67]$. The guard wires create an inactive drift cell and as a result, the overall chamber thickness needed for a given number of cells to be traversed by a particle track is increased. Thus, a higher operating voltage for a given average field is required, while a moderate operating voltage is prudent in the humid coastal Virginia climate.

Instead of using guard wires, the Hall AVDCs sandwich the sense wires between two cathode planes. Fig. 4.3 shows a cross section of the sense region for a single wire plane; the distance between two cathode plane is $26 \mathrm{~mm}$ and the sense wires are spaced 4.24 

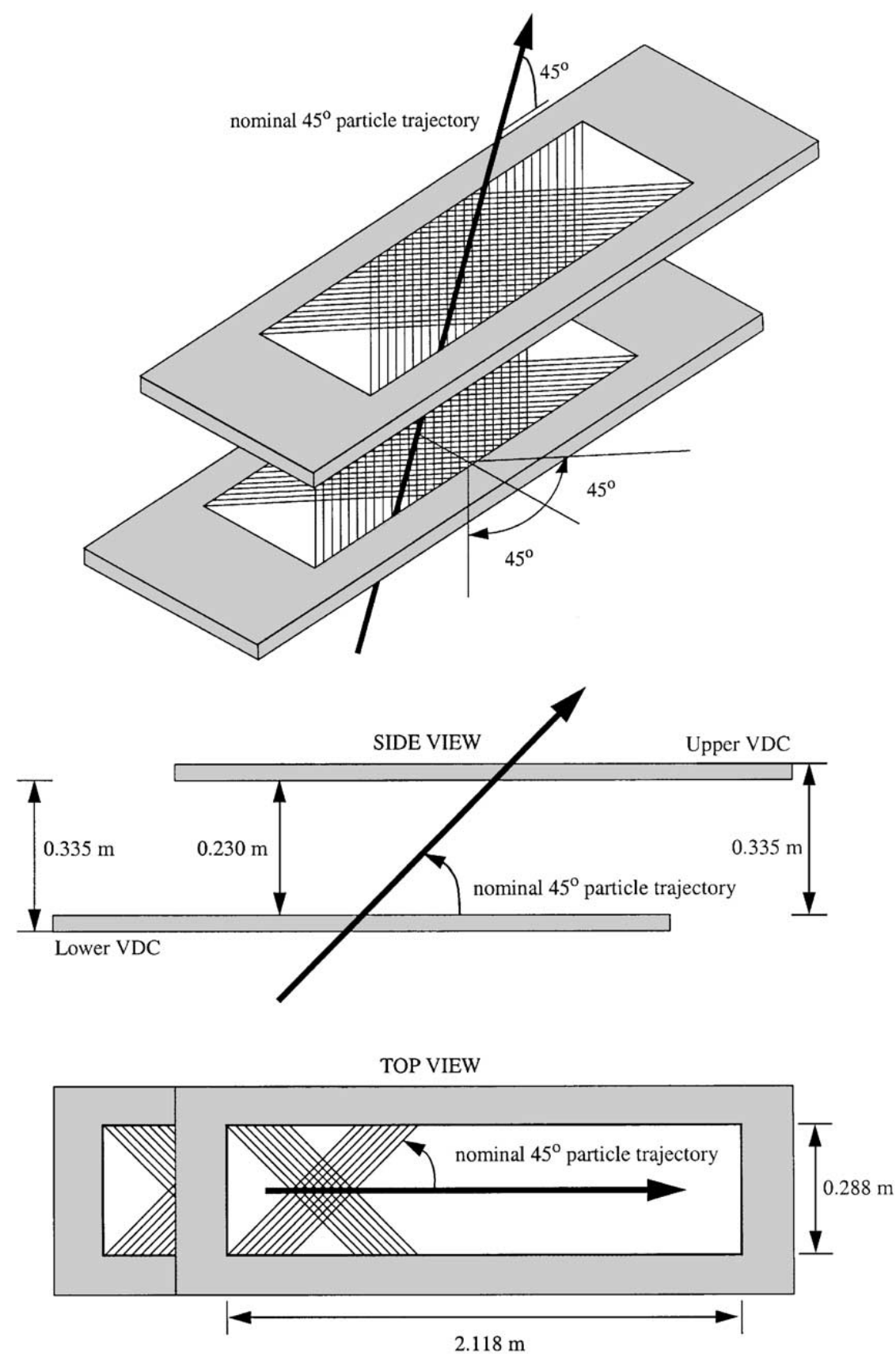

FIG. 4.2: Schematic layout of the VDCs (not to scale). The rectangular area of each wire frame aperture is $2.118 \mathrm{~m} \times 0.228 \mathrm{~m}$. The $\mathrm{U}$ and $\mathrm{V}$ sense wires are orthogonal to each other and lie in the horizontal plane of laboratory. They are inclined at an angle of $45^{\circ}$ with respect to both dispersive and non-dispersive directions. The lower VDC coincides (essentially) with the spectrometer focal plane. The vertical offset between the wire planes is $0.335 \mathrm{~m}$. Figure is from Ref. [65]. 
mm apart. A negative high voltage is applied to the cathode planes and the sense wires are grounded, which produces the electric field lines as shown by the black arrow lines in Fig. 4.3. The chambers are filled with a gas mixture of argon (62\%) and ethane (38\%). There are a total of 400 wires for each plane, spaced $4.24 \mathrm{~mm}$ apart, 368 of which are sense wires. The remaining 16 wires at each end of the wire plane have gradually increasing diameters and decreasing lengths for field-shaping purposes. The nominal trajectory angle for the HRS in the spectrometer mid-plane (see Fig. 4.2) is $45^{\circ}$, and it corresponds to the angle of $52^{\circ}$ in a plane perpendicular to the sense wires given the orientation of the wires with respect to the spectrometer mid-plane. For a typical track, as shown in Fig. 4.3, the charged particle passes through the chamber as shown by the red arrow and produces electron and ion pairs along its path. The ionized electrons drift along the electric field lines (magenta arrows), which is the path that takes the least time for the ionized electrons to travel from the trajectory to the sense wire, and a typical track fires an average of five sensor wires (orange dots in Fig. 4.3). A negative analog signal is collected at the sense wire and transmitted to the read-out card, where the signal is amplified and discriminated and a logic output is produced.

\subsubsection{Straw Chamber (SC)}

Experiment E12-07-108, also called the GMp experiment, used VDCs to obtain the tracking information of the scattered electrons in each spectrometer arm. By analyzing the data from the VDCs, the electron's momentum and trajectory angle can be calculated with high precision for one-track events at the focal plane: the cross-over point (the middle black cross in Fig. 4.3) on each wire plane is determined first and then the tracking information is calculated based on the cross-over points. For multiple-track events, the smallest $\chi^{2}$ method is used to get the valid tracks: there could be multiple cross-over points on each 


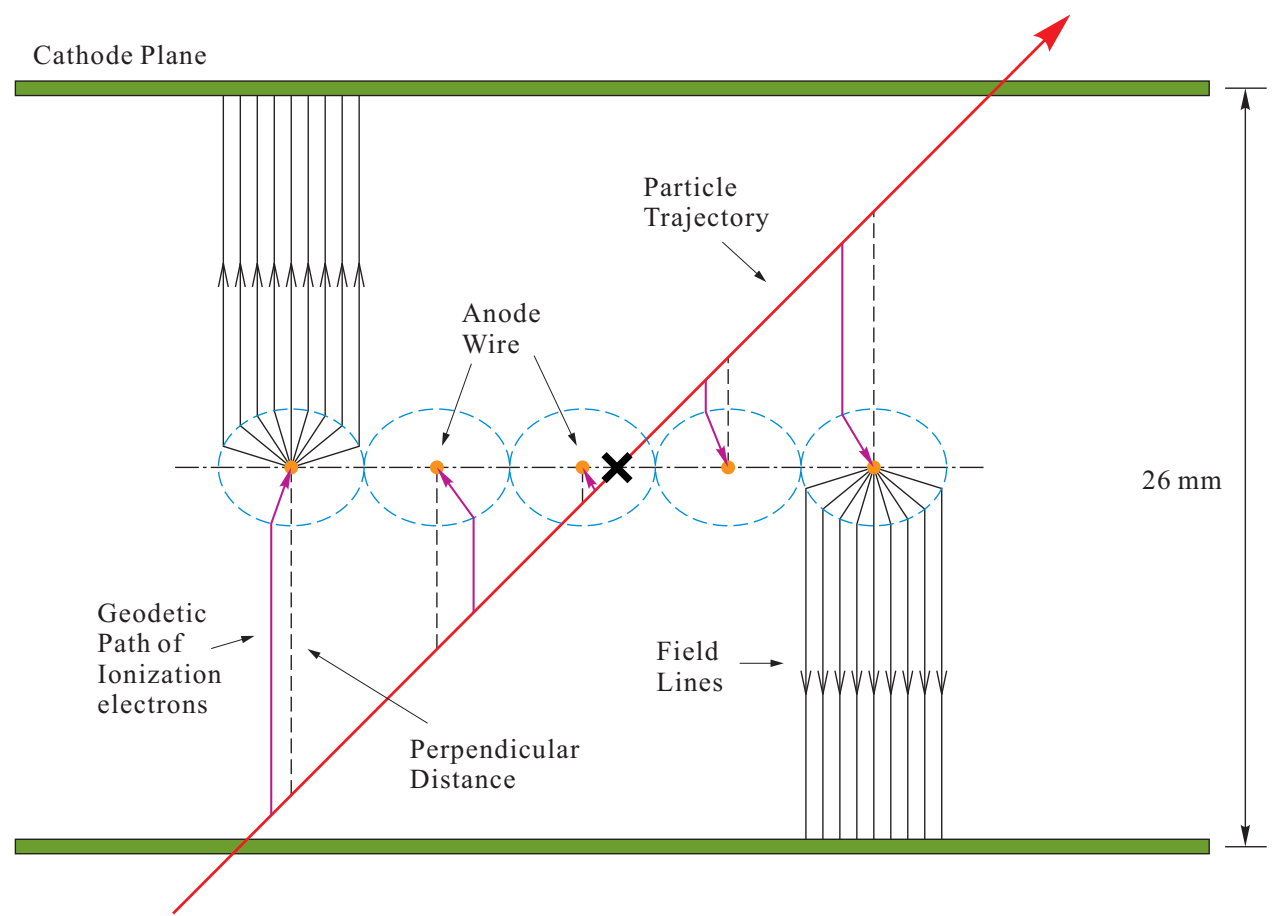

FIG. 4.3: Configuration of the wire chambers in the VDCs [3] and a typical 5-cell track. The magenta arrowed lines are paths of least time for the ionization electrons to travel from the trajectory to the sense wires. The dot/dashed lines are the corresponding projection distances used to reconstruct the trajectory. The ellipses represent the regions near the wires where the field lines make a transition from parallel to radial. 
wire plane or on one wire plane. The valid combination of points is determined by the smallest $\chi^{2}$ method.

To obtain more information about the multi-tracks events and to determine the valid tracks more accurately, experiment E12-08-108 took advantage of the existing focal plane polarimeter (FPP) [60] of Hall A and installed one straw chamber (SC) downstream from the VDCs on each spectrometer. The FPP consists of one analyzer and four tracking detectors: two front straw chambers and two rear straw chambers. For experiment E1207-108, we modified, upgraded and tested the two front straw chambers and installed one on each HRS arm. The two straw chambers are the same.

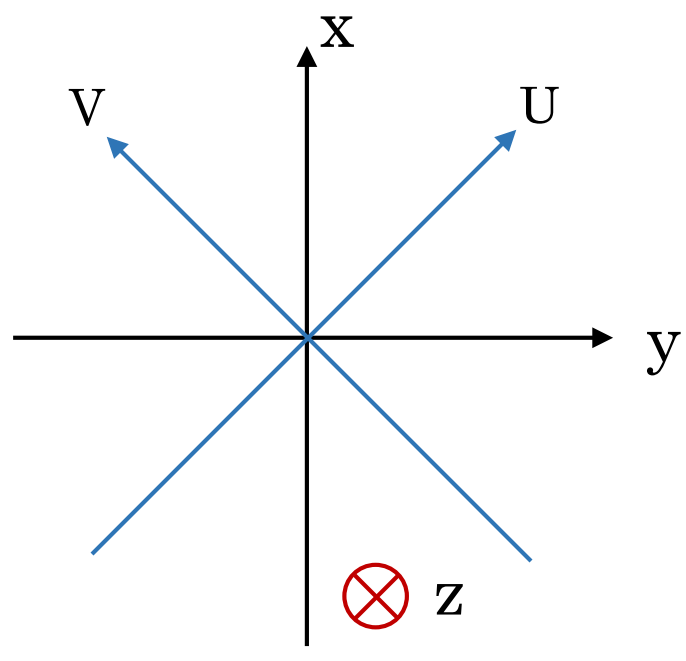

FIG. 4.4: The coordinate system of the straw chamber. Axis $z$ is along the central ray direction of the spectrometer and $x$ is along the dispersive direction.

As shown in Fig. 4.1, the SC on each spectrometer was located after the VDCs and followed by $\mathrm{S}_{0}$ (see Chap. 4.2.3). The $\mathrm{SC}$ was oriented perpendicular to the central ray direction with the longer side along the dispersive direction. The SC is a drift chamber composed of a set of straw tubes, which are cylindrical tubes with a thin wire running along the central axis of each tube. The inner diameter of each tube is $10.44 \mathrm{~mm}$ and it is 
filled with a gas mixture of $62 \% / 38 \%$ argon/ethane by volume. The tubes are grounded while the central anode wire is at a high voltage of $+1800 \mathrm{~V}$ when the SC is operated.

The principle of how SC works is similar to that of VDCs: the charged particles go through the SC tube (drift chambers for VDCs) and the produced electrons and ions travel to the anode wire and the tube shell (cathode planes for VDCs) respectively. Under the typical working conditions of the SC at Jefferson Lab, the drift velocity of the electrons is $\sim 50 \mu \mathrm{m} / \mathrm{s}$. When the electrons get within about $100 \mu \mathrm{m}$ of the wire, the electric field strength is large enough for an electron avalanche to happen. A gain of about $10^{5}$ per primary ionization is produced and a negative analog signal is collected at the wire. The analog signal is then sent to a read-out board, where it is amplified and discriminated and a logic output is produced. Different from VDCs, each anode wire is located in a tube filled with a gas mixture and a set of tubes are installed together for the SC while the sense wires in VDCs are all in one drift chamber.

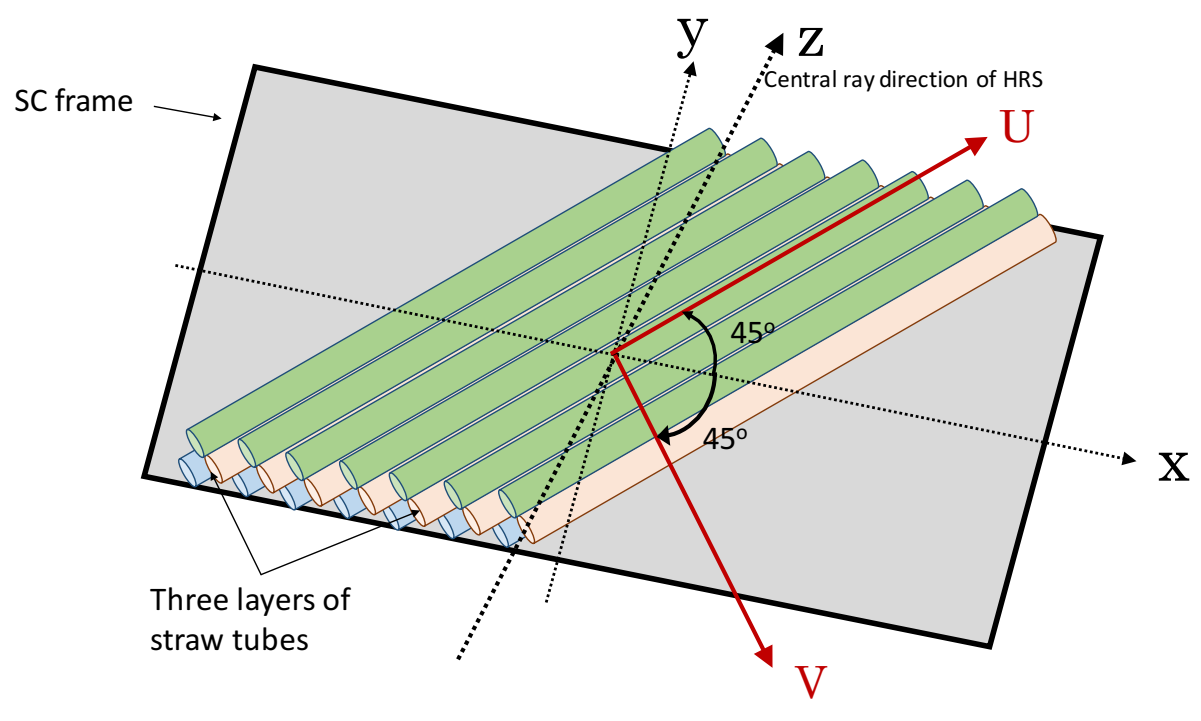

FIG. 4.5: The schematic layout of the three U planes of tubes for SC. $z$ is along the central ray direction and $x$ is along the dispersive direction of HRS. There are three $\mathrm{V}$ planes of tubes at the bottom of the SC frame not shown on the graph. 
One straw chamber contains 6 planes: $3 \mathrm{U}$ planes and $3 \mathrm{~V}$ planes. In the $\mathrm{SC}$ coordinate system, $z$ is along the spectrometer central ray direction, $x$ is along the dispersive direction and $y$ is along the non-dispersive direction of the HRS. The $\mathrm{U}$ and $\mathrm{V}$ directions are at $+45^{\circ}$ and $-45^{\circ}$ relative to the dispersive direction (see Fig. 4.4) in the $x-y$ plane.

In the $\mathrm{U}$ and $\mathrm{V}$ planes, the tubes are along the $\mathrm{U}$ and $\mathrm{V}$ directions respectively. There are 176 tubes in each U plane and 160 tubes in each V plane. The planes are in a UUUVVV configuration, three $\mathrm{U}$ planes of tubes on the top of the frame and three $\mathrm{V}$ planes of tubes at the bottom of the frame (see Fig. 4.5). The V planes are closer to the VDCs.

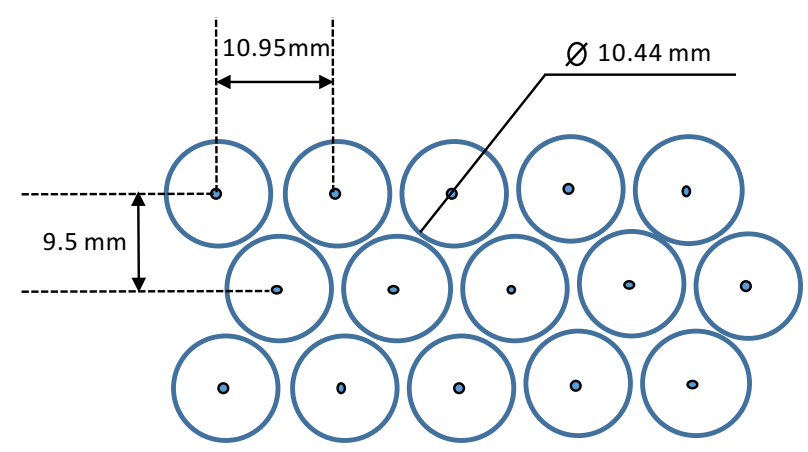

FIG. 4.6: Tubes configuration in three U planes or $\mathrm{V}$ planes in the straw chamber.

As shown in Fig. 4.6, the tubes in each plane are precisely spaced $10.95 \mathrm{~mm}$ apart, the middle layer of tubes are shifted by half a hole separation and the vertical distance between two layers is $9.5 \mathrm{~mm}$. The active area of the straw chamber is $209 \mathrm{x} 60 \mathrm{~cm}^{2}$.

\subsubsection{Plastic Scintillators and Triggers}

During experiment E12-07-108, there was one $\mathrm{S}_{0}$ scintillator plane before and one $\mathrm{S}_{2 m}$ scintillator plane after the Gas Cherenkov on each spectrometer in Hall A, providing triggering and timing information (see Fig. 3.4). The two scintillators planes are parallel to each other and about 1.6 meters apart. The two scintillators planes are perpendicular 
to the nominal central ray of the spectrometer.

Scintillator $\mathrm{S}_{0}$ plane contains one thin long scintillator paddle with one photo-multiplier tube (PMT) attached to the top and one to the bottom end to collect the photons produced by the particles passing through the scintillator. Fig. 4.7 shows scintillator $\mathrm{S}_{0}$ configuration. The active area of $\mathrm{S}_{0}$ is $\sim 1.7 \mathrm{~m} \times 0.25 \mathrm{~m}$.

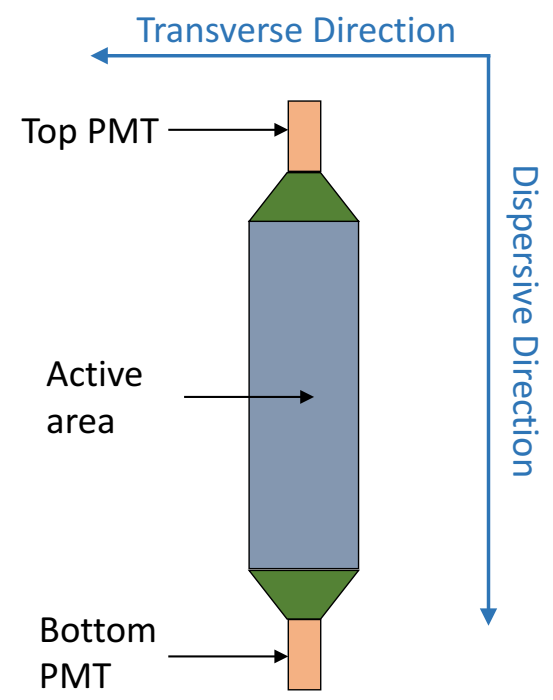

FIG. 4.7: Scintillator $\mathrm{S}_{0}$ plane configuration. The scintillator plane is perpendicular to the nominal central ray of the spectrometer.

Scintillator $\mathrm{S}_{2 m}$ plane has 16 paddles and each paddle is attached to two PMTs, one at each end. We refer to these PMTs as the left and right PMT of the scintillator plane. Fig. 4.8 shows scintillator $\mathrm{S}_{2 m}$ configuration. The active area of $\mathrm{S}_{2 m}$ is $\sim 2.236 \mathrm{~m} \times 0.432$ m.

The DAQ (see Chap. 4.2.6) readout is controlled by the trigger supervisor which receives the trigger signals. For the $\mathrm{S}_{0}$ plane, a coincidence between the top nd bottom PMTs was made. For the $\mathrm{S}_{2 m}$ plane, the logical OR of the PMTs was formed individually for the left 16 PMTs and the right 16 PMTs and then the logical AND was formed between 


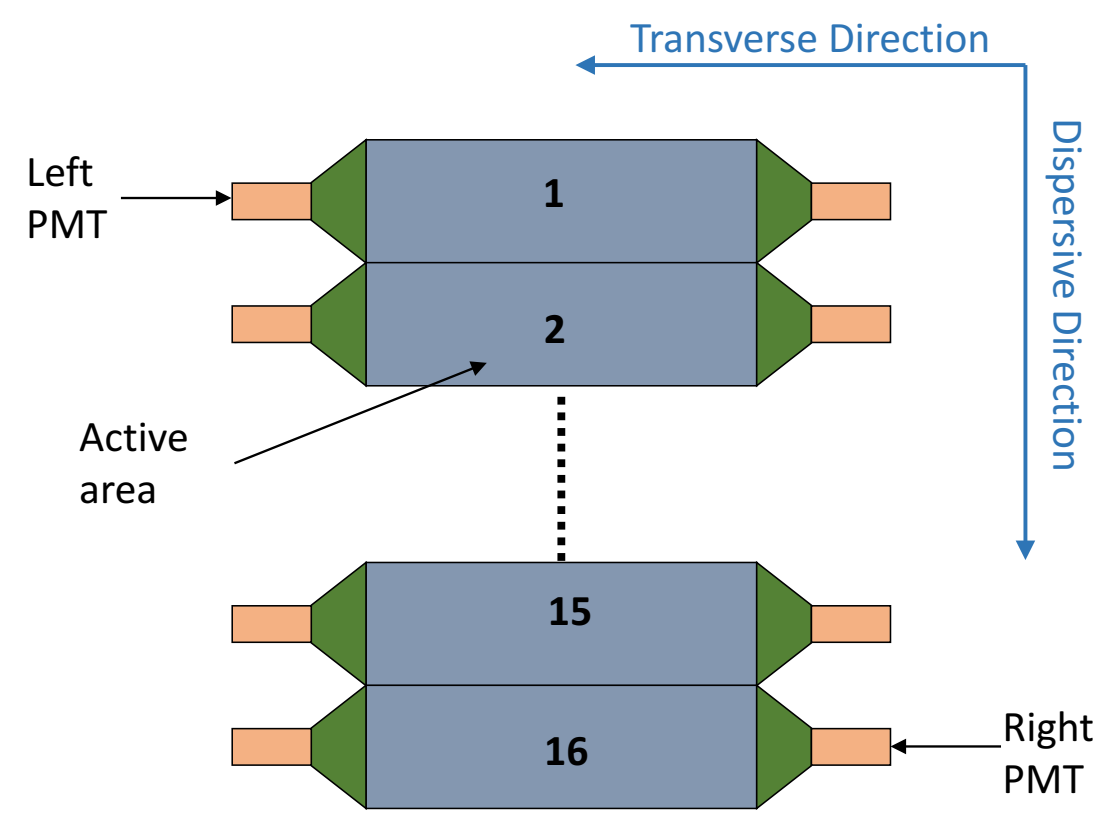

FIG. 4.8: Scintillator $S_{2 m}$ plane configuration. The scintillator plane is perpendicular to the nominal central ray of the spectrometer.

them. This means at least one of the left PMTs and one of the right PMTs needs to be hit to form the $\mathrm{S}_{2 m}$ trigger signal. For the gas Cherenkov detector (see Chap. 4.2.4) the analog sum of the signals from its 10 PMTs was used to prepare a hit signal.

The main trigger for each spectrometer of the experiment E12-07-108 was formed by the logical AND of the $S_{0}$ and $S_{2 m}$ planes, which is called T1. To measure the trigger efficiency of $\mathrm{T} 1$, an alternative trigger was formed by the coincidence of scintillator $\mathrm{S}_{2 m}$ and the gas Cherenkov detector, which is called T3. However, both T1 and T3 contain the signals from $\mathrm{S}_{2 m}$ thus at least one more trigger independent of $\mathrm{S}_{2 m}$ is needed to determine T1 efficiency. 


\subsubsection{Gas Cherenkov Detector}

In order to separate the electrons from background particles such as negatively charged pions, a Cherenkov detector filled with $\mathrm{CO}_{2}$ at atmospheric pressure [68] was installed between the scintillator $S_{0}$ and $S_{2 m}$ planes on each arm for particle identification.

The Gas Cherenkov detector is based on the detection of Cherenkov light, which is produced when a high energy charged particle travels through a material of index of refraction $n$ with velocity $v$ larger than the velocity of light, $c / n$, in that material, where $c$ is the speed of light. The Cherenkov light is emitted only when the velocity of the charged particles is larger than a threshold:

$$
v_{t h}=\frac{c}{n}
$$

and therefore a threshold momentum can be calculated:

$$
p_{t h}=\frac{m v_{t h}}{1-\left(\frac{v_{t h}}{c}\right)^{2}}
$$

The index of refraction of atmospheric pressure $\mathrm{CO}_{2}$ is 1.00041, which gives an electron momentum threshold $\sim 17 \mathrm{MeV}$ and a pion momentum threshold $\sim 4.8 \mathrm{GeV}$. The pion momentum threshold is higher than the HRS momentum range so only the electrons will trigger the detector. However, sometimes pions will still produce a Cherenkov light if the pions knock out electrons with momentum higher than the threshold when they pass through material before getting into the Cherenkov detector.

The HRS Cherenkov detector contains ten spherical mirrors that reflect the Cherenkov radiation onto a set of 10 PMTs. The signals from the PMTs are then sent to analog-

to-digital converters (ADC) and summed. Knock-out electrons with sufficient energy also contribute to the ADC signals and these events are removed with the help of the electromagnetic calorimeter. 


\subsubsection{Electromagnetic Calorimeter}

The electromagnetic calorimeter on each arm of the HRS provides additional information for particle identification. The calorimeter measures the energy deposited by a charged particle by pair production and Bremsstrahlung radiation. When a high energy particle travels through a dense material, it emits photons through Bremsstrahlung and these photons create electron-positron pairs which radiate more photons. This process is repeated and as a result a shower of electrons, positrons and photons will be generated. This is the reason that the electromagnetic calorimeter is called the shower counter. Electrons and positrons produce Cherenkov light which is detected by the PMTs at the end of each lead-glass block of the calorimeter. Electrons and positrons deposit all of their energy in the detector, while hadrons, mainly pions, usually deposit a small amount of energy due to ionization and direct Cherenkov light.

The Hall A electromagnetic calorimeter on each spectrometer arm contains two layers of lead-glass detectors, as shown in Fig. 4.9. The particles enter the detector through the bottom of the figure. The blocks in both layers in HRS-L and in the first layer in HRS-R are oriented perpendicular to the particle tracks. The blocks are parallel to the tracks in the second layer of HRS-R [60]. The HRS-R shower detector is a total absorption calorimeter, since it is sufficiently thick to contain the entire shower for the spectrometer's momentum range.

\subsubsection{Data Acquisition (DAQ)}

The data acquisition system (DAQ) in Hall A was used during experiment E12-07-108, which is controlled by the CEBAF Online Data Acquisition System (CODA) [4]. CODA is a suite of software tools and components which can be used to implement DAQ with nuclear physics data taking. Fig. 4.10 shows a simple CODA implementation. 

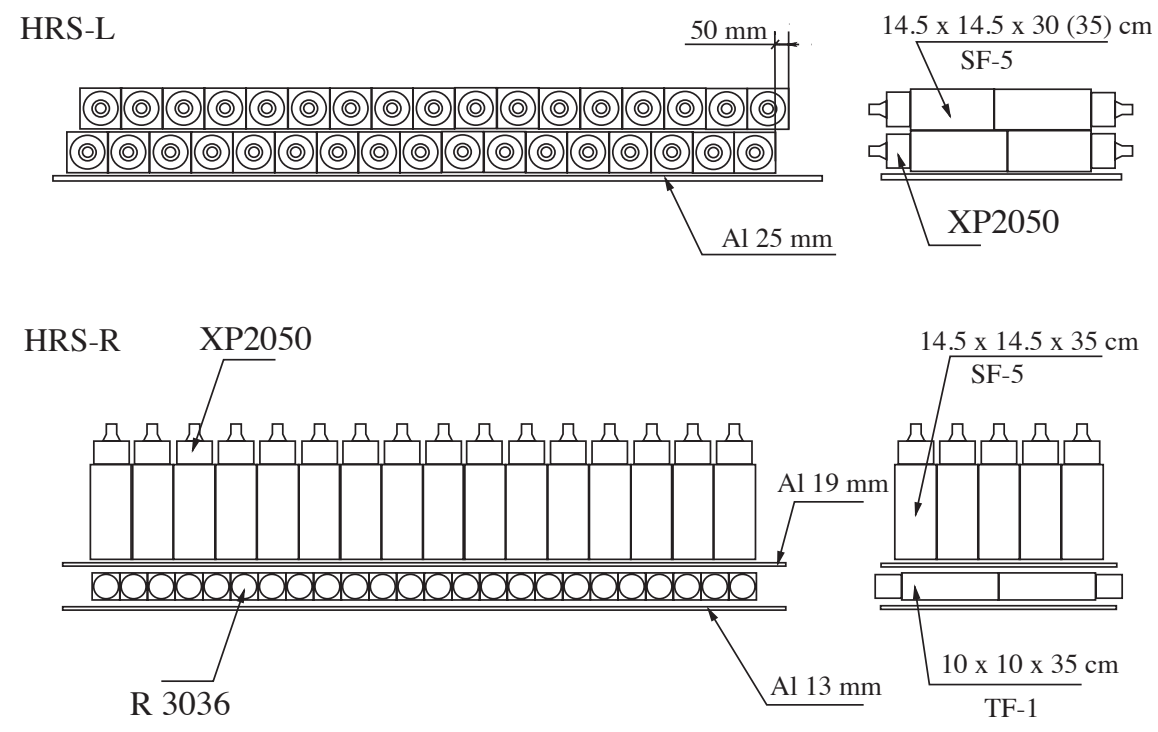

FIG. 4.9: Schematic layout of part of the shower detectors in HRS-L (top) and HRS-R (bottom) Particles enter from the bottom of the figure. Figure is from Ref. [60].

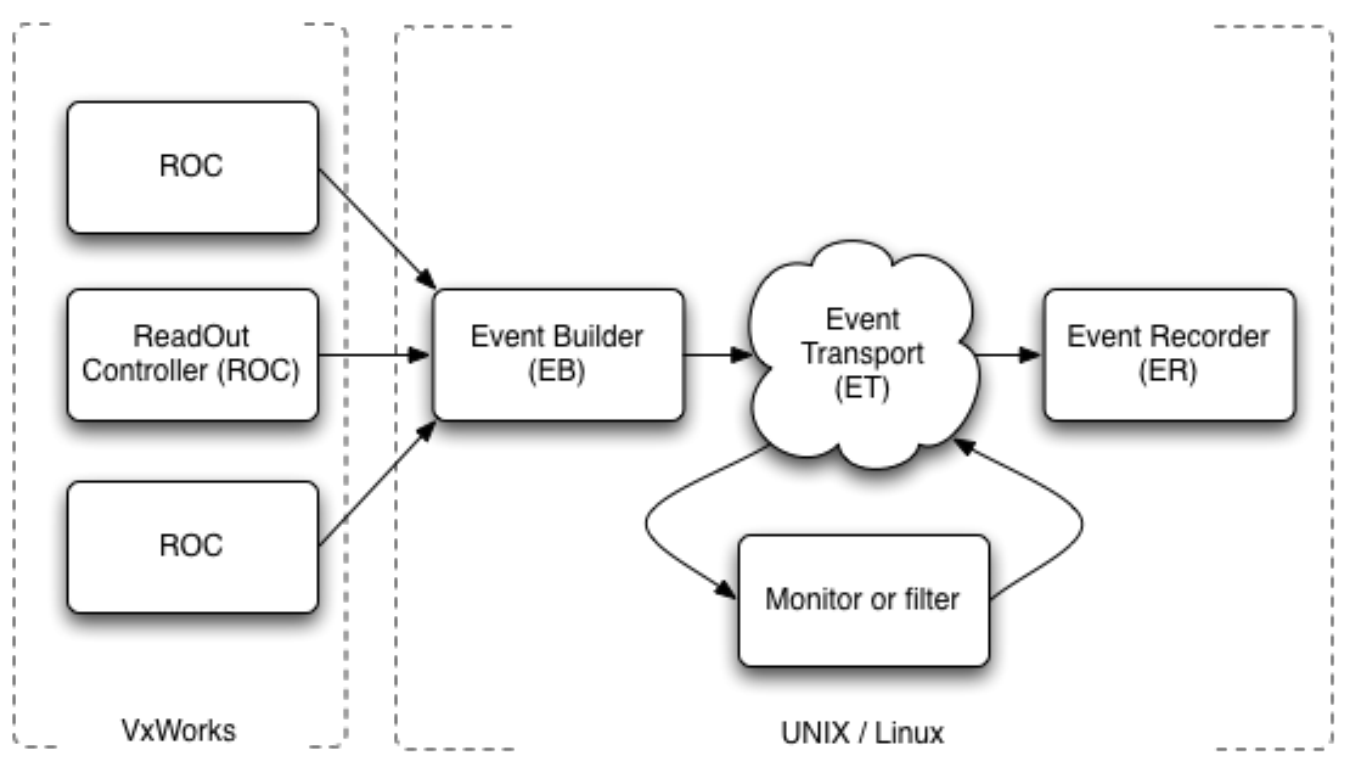

FIG. 4.10: A simple CODA implementation. [4]. 
The data from ADCs, TDCs and scalers is read out from the Read-out Controllers (ROCs), which are CPUs in the FASTBUS and VME crates. The Trigger Supervisor controls the state of the run and generates the triggers to start the ROCs to read out. Then, the ROCs send the data event fragments to the Event Builder (EB), where the data is put into an "event" with the header information describing the event. After the event is built by the EB, it is placed into a buffer and then tested. The accepted events are recorded by the event recorder and written to a local disk and finally transferred to the Mass Storage System. CODA also contains a graphical user interface (RunControl) which allows the user to start and stop runs and change run parameters. There are three types information: physics events from the spectrometers (detectors), beamline information and Experimental Physics Industrial Control System (EPICS) [69] information. EPICS quantities include the spectrometer angles, magnets, target controls, etc.

\subsection{Spectrometer Optics}

The HRS optics offers a method to obtain the tracking information of the scattered particles at the target, including the reaction vertex, scattering angle and momentum. The focal plane coordinate variables of the detected particles are calculated by a pair of VDCs (see section 4.2.1). The optics matrix elements allow the reconstruction of the reaction variables at the target from the focal plane coordinate variables. This section describes the standard calibration procedure to determine the optics matrix elements.

\subsubsection{Coordinate Systems}

In this section, an overview of the target and focal plane coordinate system will be presented. A detailed description of the coordinate systems is included in [70]. When a particle is detected by the VDCs, two angular coordinates $\left(\theta_{\text {det }}\right.$ and $\left.\phi_{\text {det }}\right)$ and two spatial 
coordinates $\left(x_{d e t}\right.$ and $\left.y_{d e t}\right)$ are measured at the focal plane. The position of the particle and the tangent of the angle made by its trajectory along the dispersive direction are denoted by $x_{\text {det }}$ and $\theta_{\text {det }}$, while $y_{\text {det }}$ and $\phi_{\text {det }}$ represent the position and tangent of the angle along the non-dispersive direction [70]. These detector coordinate system variables, $\theta_{\text {det }}$, $\phi_{\text {det }}, x_{\text {det }}$ and $y_{\text {det }}$, are transferred to the focal plane coordinate system variables including the corrections for the offsets incurred due to misalignments in the VDC packages. Then these focal plane variables are used to calculate the scattering angle, reaction vertex and relative momentum $\left(\theta_{t g}, \phi_{t g}, y_{t g}\right.$ and $\left.\delta\right)$ using the optics matrix elements. $\delta$ is defined as

$$
\delta=\frac{P-P_{0}}{P_{0}}
$$

where $P$ is the particle's measured momentum and $P_{0}$ is the central momentum of the spectrometer.

Each of the two spectrometers has its own target coordinate system (TCS). Figure Fig. 4.11 shows the TCS for the left arm spectrometer. A line perpendicular to the sieve slit surface of the spectrometer and going through the midpoint of the central sieve slit hole defines the $z$ axis of the TCS for a given spectrometer. The $z$ axis is pointing away from the target to the sieve. The sieve slit is a block with holes used to calibrate optics matrix elements. In the ideal case, the spectrometer and sieve slit offsets are zero so that the spectrometer central ray goes though the hall center, the sieve slit sits right in the center of the spectrometer, and the $z$ axis of the TCS passes through the hall center. For this case, the distance from the hall center to the center of the sieve slit is $L$. The origin of the TCS is defined to be the point on the $z$ axis at a distance $L$ from the sieve surface to the direction of the hall center. In the non-ideal case, there is a horizontal offset of the spectrometer from the hall center, D, and then the origin of TCS is as shown in Fig. 4.11. The $y_{t g}$ axis is parallel to the sieve surface in the horizontal direction and $x_{t g}$ is vertically 
down. The tangent of the out-of plane angle and in-plane angle are given by $\theta_{t g}$ and $\phi_{t g}$ respectively.

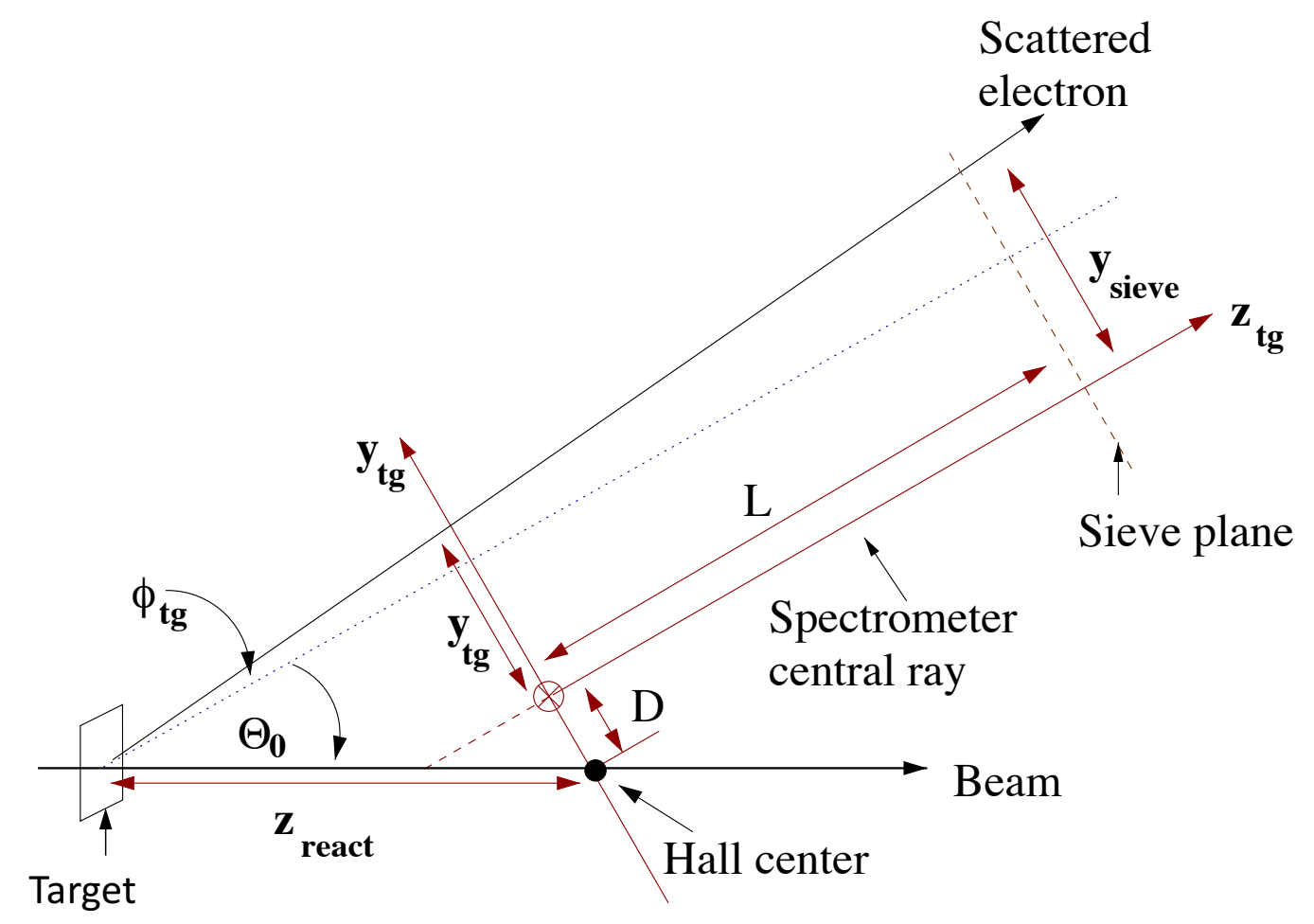

FIG. 4.11: Target coordinate system (TCS) for a electron scattering from a thin foil target. 


\subsubsection{Optimization Method}

The optics matrix links the focal plane coordinates to the target coordinates. The relationship between them for the first-order approximation is written as:

$$
\left[\begin{array}{l}
\delta \\
\theta \\
y \\
\phi
\end{array}\right]_{t g}=\left[\begin{array}{cccc}
\langle\delta \mid x\rangle & \langle\delta \mid \theta\rangle & 0 & 0 \\
\langle\theta \mid x\rangle & \langle\theta \mid \theta\rangle & 0 & 0 \\
0 & 0 & \langle y \mid y\rangle & \langle y \mid \phi\rangle \\
0 & 0 & \langle\phi \mid y\rangle & \langle\phi \mid \phi\rangle
\end{array}\right]\left[\begin{array}{c}
x \\
\theta \\
y \\
\phi
\end{array}\right]_{f p}
$$

The mid-plane symmetry of the spectrometer requires the null matrix elements. In practice, a set of tensors, $Y_{j k l}, T_{j k l}, P_{j k l}$ and $D_{j k l}$, link each target coordinate to the focal plane coordinates. These tensors are polynomials in $x_{f p}$. For example,

$$
\begin{gathered}
\theta_{t g}=\sum_{j, k, l} T_{j k l} \theta_{f p}^{j} y_{f p}^{k} \phi_{f p}^{l} \\
T_{j k l}=\sum_{i=0}^{m} C_{i} x_{f p}^{i}
\end{gathered}
$$

The expressions for $\phi_{t g}, y_{t g}$ and $\delta$ are similar and the $C_{i}$ are the optics matrix elements for the corresponding target variable. The mid-plane symmetry of the spectrometer requires that for $Y_{j k l}$ and $P_{j k l},(k+l)$ is odd, while for $D_{j k l}$ and $T_{j k l},(k+l)$ is even. These indices are the power of the focal plane variables and for experiment E12-07-108, the matrix was optimized up to fourth order.

The matrix elements are determined by $\chi^{2}$ minimization of the aberration functions,

$$
\Delta(W)=\sum_{s}\left[\frac{W-W^{0}}{\sigma_{W}^{s}}\right]^{2}
$$


where $W$ is $\theta_{t g}, \phi_{t g}, y_{t g}$ or $\delta$. This process compares the reconstructed events $(W)$ to the nominal target foil and sieve slit positions $\left(W^{0}\right)$, which are known from the experiment's surveys, and set $\Delta p / p$. The reaction vertex position along the beam $z_{\text {react }}$, the vertical and horizontal positions at the sieve, $x_{\text {sieve }}$ and $y_{\text {sieve }}$ can be calculated from the reconstructed target variables as follows:

$$
\begin{gathered}
z_{\text {react }}=-\left(y_{t g}+D\right) \frac{\cos \left(\arctan \phi_{t g}\right)}{\sin \left(\Theta_{0}+\arctan \phi_{t g}\right)}+x_{\text {beam }} \cot \left(\Theta_{0}+\arctan \phi_{t g}\right) \\
x_{\text {sieve }}=x_{t g}+L \theta_{t g} \\
y_{\text {sieve }}=y_{t g}+L \theta_{t g}
\end{gathered}
$$

Note that $\theta_{t g}$ and $\phi_{t g}$ are the tangent of the out-of plane angle and in-plane angle, respectively. 


\section{CHAPTER 5}

\section{GMp High Resolution Spectrometer (HRS) Improvement \& Calibration}

\subsection{Detector Packages Improvement}

\subsubsection{VDC Improvement}

The principle and properties of VDCs, which are used for tracking information on both spectrometers, were introduced in Chap. 4.2.1. The tracking information is obtained by analyzing the data from the VDCs. The ionized electrons drift towards the sense wires and are collected as analog pulse signals. Analog signals from 16 sense wires are transmitted respectively to a 16-channel preamp/discriminator cards (A/D cards), where the signals are first amplified and then discriminated. The A/D cards produce logic outputs and these signals are sent to multi-hit time to digital converters (TDCs) after processing by a level translator. The logic of the read-out system is shown in Fig. 5.1.

The GMp experiment, the hardware of VDCs were upgraded and tested: new preamp/disc 


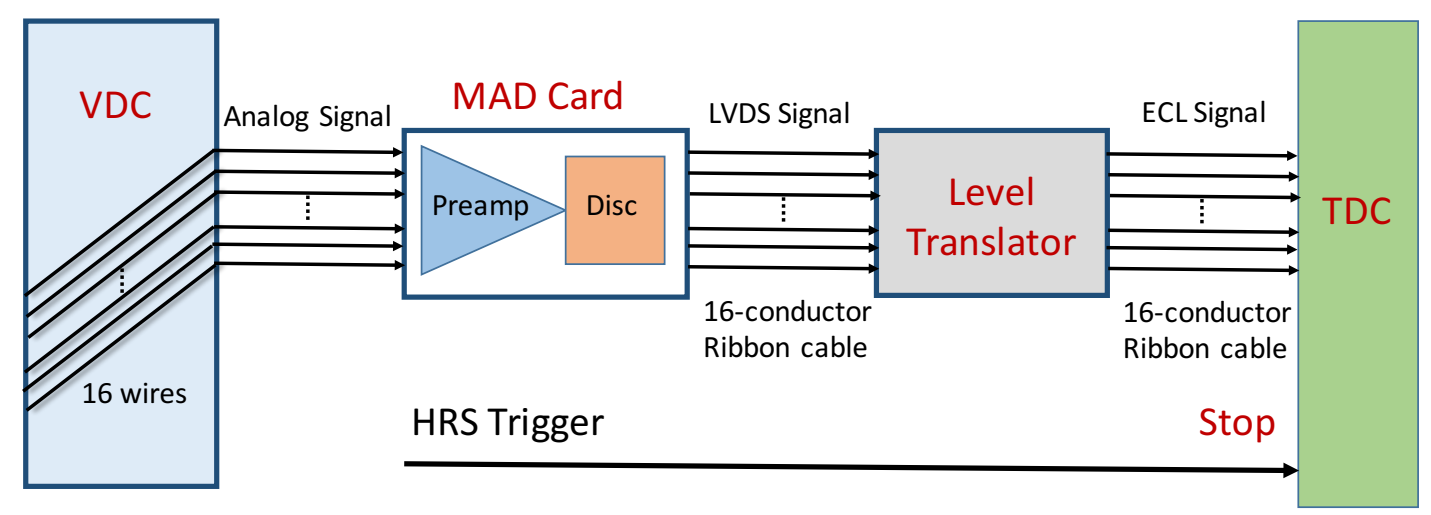

FIG. 5.1: Block diagram for the logic of the VDC signal. (l.e. = leading edge, t.e. $=$ trailing edge).

cards (A/D cards), low voltage and threshold power supplies, level translators and their power supply, were all installed.

\section{Preamp/disc cards}

When operated, the sense wires of VDCs are connected to ground potential and the anode planes are at negative high voltage. Due to the electric field produced between the wires and the planes, when a charged particle passes through the chamber of the VDCs, the ionized electrons drift towards the sense wires and an electron avalanche happens when the electrons get close to the wire. The wire is fired and an analog signal is transmitted to the A/D cards, where the signal is amplified and discriminated and then a logic output is produced. Previously, LeCroy 2475 type cards were used in the VDCs. These cards require a minimum threshold of $5 \mu \mathrm{A}$, which requires a working high voltage of $-4 \mathrm{kV}$ for the VDCs. This limits the maximum rate for stable operation of VDCs because of space effects.

When the ionized electrons drift towards the sense wires, the positive charged ions move slowly towards the high voltage planes. They partially shield the sense wire and thus 
diminish the electric field and the gas amplification, which is know as the space charge effect. To minimize the influence of the space charge effect, the gas amplification should be kept low. One way to achieve this goal is to reduce the gain and increase the corresponding sensitivity of the A/D cards.

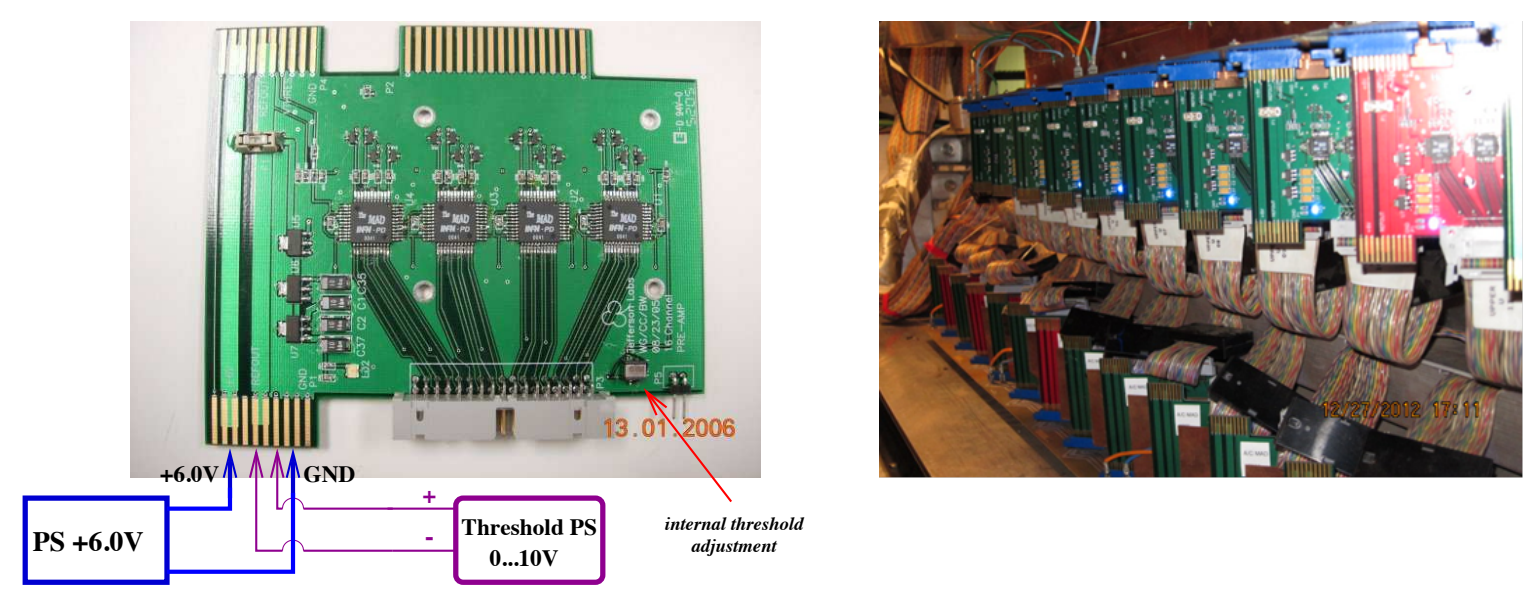

FIG. 5.2: Photograph of the MAD cards. The left panel shows one single MAD card. The right panel shows the MAD cards installed on VDCs. There are $23 \mathrm{MAD}$ cards for each VDC wire plane.

A new A/D card called the MAD card was developed by JLab's Electronics Group with the charge-sensitive input stage of MAD being several times less noisy than the current-sensitive LeCroy card. The MAD's output stage works with LVDS signals which are known to produce significantly less feedback than the ECL signals used in the LeCroy cards. These features of the new cards makes it feasible to set a threshold significantly lower than is achievable with the LeCroy cards. MAD cards increase the signal sensitivity by a factor of five over the LeCroy cards, which directly translates to an additional factor of five in rate capability, bringing the VDC rate limit to $10 \mathrm{MHz}$ [71]. This increased sensitivity of the MAD cards allows them to operate with a decreased threshold and thus allows the VDCs to operate at $-3.5 \mathrm{kV}$ instead of $-4 \mathrm{kV}$. The reduced high voltage makes long term stable operation possible without aging while using the standard VDC gas 
mixture.

For experiment E12-07-108, we installed the new MAD cards on the VDCs on both arms of HRS. There are 368 sense wires in each VDC plane and thus there are 23 MAD cards installed for each plane. Fig. 5.2 shows the MAD cards installed on VDCs.

After the MAD cards were installed, they were checked by studying their efficiency with cosmic runs. The algorithm used to estimate and track the wire efficiency is straightforward. A given wire plane is scanned; if a wire is not fired but the two wires adjacent to it are fired, then this event is defined to be inefficient for this wire. The efficiency is calculated by

$$
\epsilon=\frac{\kappa}{\kappa+\lambda}
$$

where $\kappa$ is the number of times a wire is found to be efficient and $\lambda$ is the number of times a wire is found to be inefficient.

The VDCs are very efficient detectors. Ref. [65] shows the typical wire efficiency is higher than 99\%. However we found that the efficiencies for the Right-arm VDC U2 plane were much lower, only around 95\%. The most plausible reason seemed to be cross-talk between adjacent channels: when the signal is transmitted to one channel of the MAD card, if this channel triggers the adjacent ones, then there is cross-talk on this MAD card. As a result, the calculated efficiencies of the wires that are influenced by cross-talk are higher while the independent wires have lower efficiencies. When the adjacent signals are triggered by cross-talk instead of real charged particles, their drift times are the same.

To check whether cross-talk exists on these cards, the differences between the drift time for adjacent wires were examined. Fig. 5.3 gives an example of checking the 3rd card on the U2 plane of $\sum_{i=1}^{16}\left(\mathrm{TDC}_{i}-\mathrm{TDC}_{i-1}\right)$, where $\mathrm{TDC}_{i}$ is the recorded TDC value of the $i$ th channel. When cross-talk exists, $\mathrm{TDC}_{i}-\mathrm{TDC}_{i-1}=0$ and thus leads to a peak at the TDC $=0$ position. Each card on the four VDCs planes was checked and the possibility 


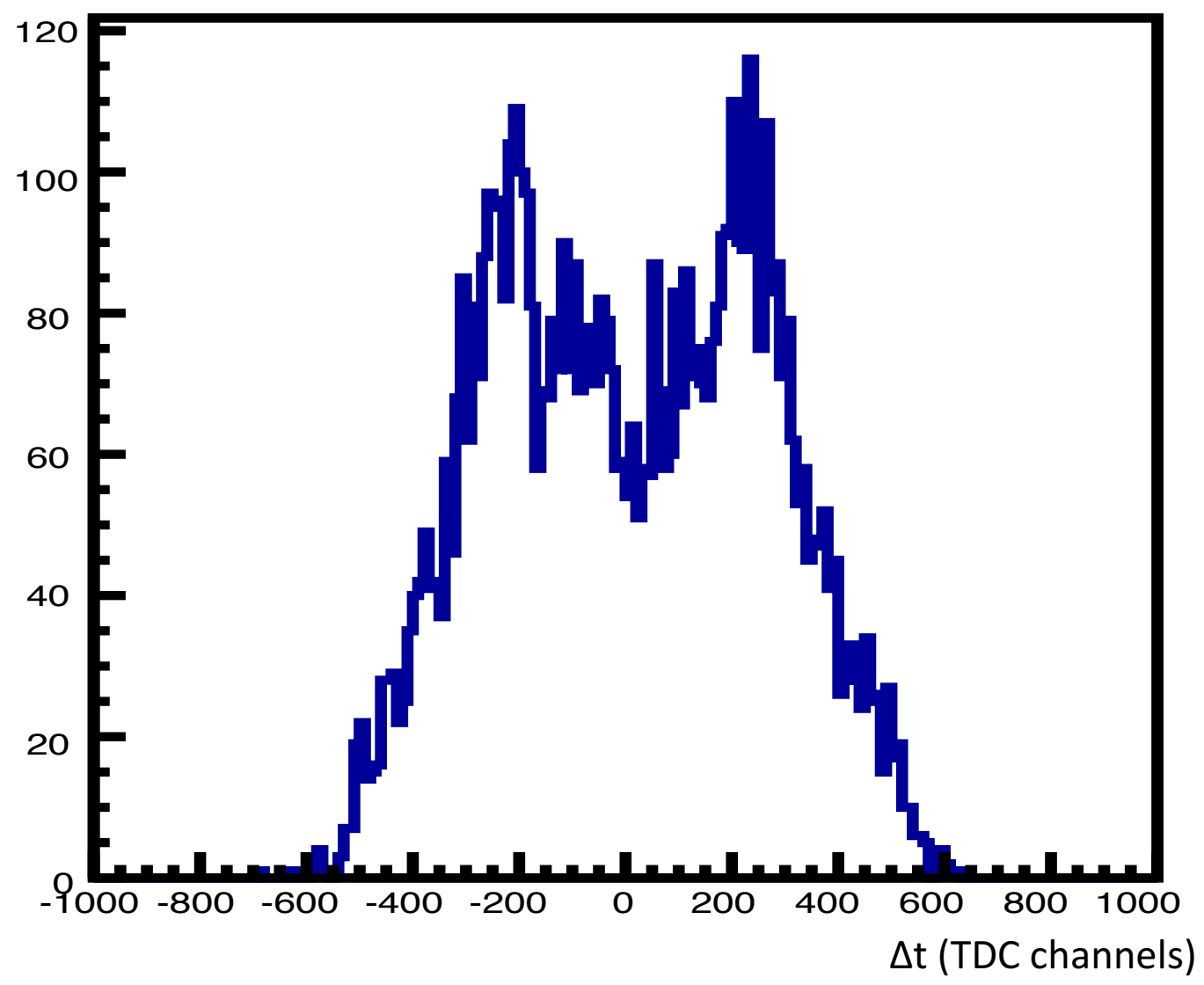

FIG. 5.3: TDC value differences between adjacent wires of the third MAD card on the U2 plane of VDCs. 
of significant cross-talk was excluded. Finally we came to the conclusion that the cards on the Right-arm U2 plane with low efficiencies needed to be replaced. Fig. 5.4 shows the wire efficiencies for 4 VDCs planes after we replaced 26 MAD cards on U2 plane. The performance of the U2 plane is improved dramatically with the cards replaced, even though it is still worse than the others.

\section{Time Resolution and HV Scan}

The algorithm used to estimate and track the inherent time resolution is also straightforward [65]. The minimum time for the ionization electrons produced in each drift cell to drift along the electric field lines to the respective sense wires is $t_{i}$, where $i=1, \ldots, 5$ for a 5-cell event (see Fig. 4.3). Then a relative timing is defined as:

$$
\Delta T=\left|\left(t_{1}-t_{2}\right)-\left(t_{5}-t_{4}\right)\right|
$$

$t_{3}$ is the drift time of the wire which is in the middle of the 5-cell, closest to the point where the trajectory crosses the wire plane. $t_{3}$ is ignored cause it does not affect the overall resolution (explained later).

Fig. 5.5 shows the relative timing $\Delta T$ for 4 planes of VDCs on the Right-arm HRS with HV being $-3.5 \mathrm{kV}$. For a perfect chamber with infinitesimal resolution, this relative timing distribution would be a $\delta$-function centered at $\Delta T=0$. Here, the FWHM of the distribution makes an estimate of $\Delta T$, the inherent per-plane time resolution. $\Delta T$ is obtained by the timing information from four TDCs connected to signal wires pairwise adjacent to the wire plane cross-over point and assuming equal contributions to the total width from each of the five drift cells traversed, the per-plane FWHM time resolution is 
Right arm U1 efficiency

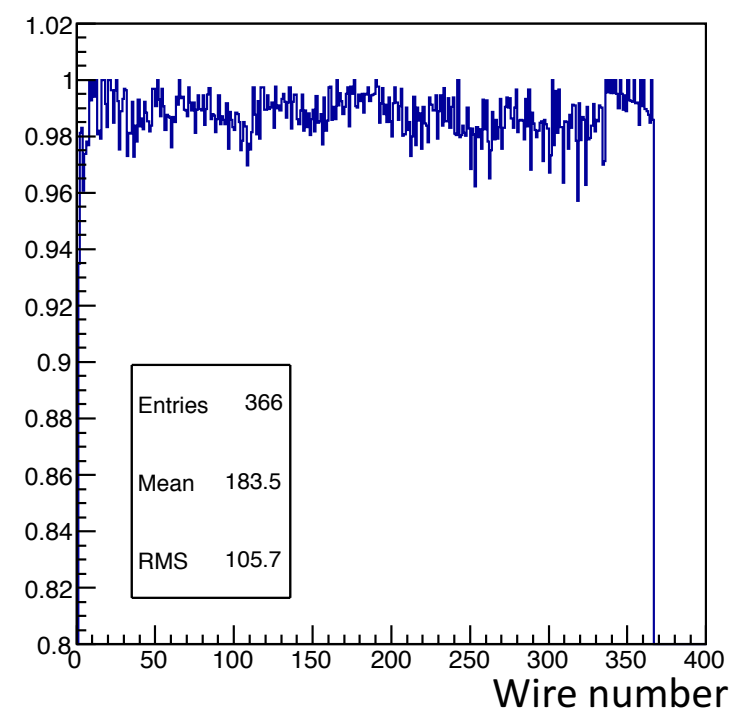

Right arm V1 efficiency

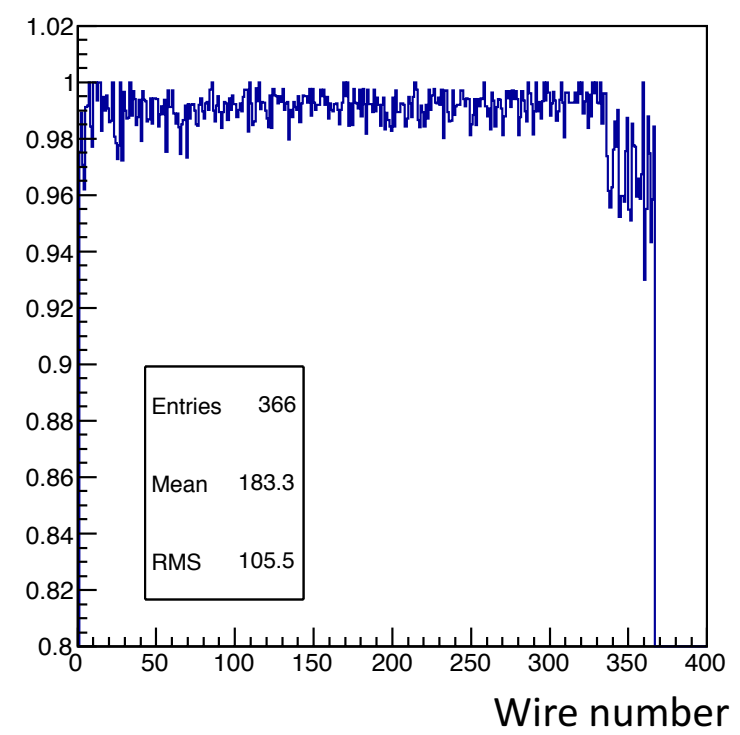

Right arm U2 efficiency

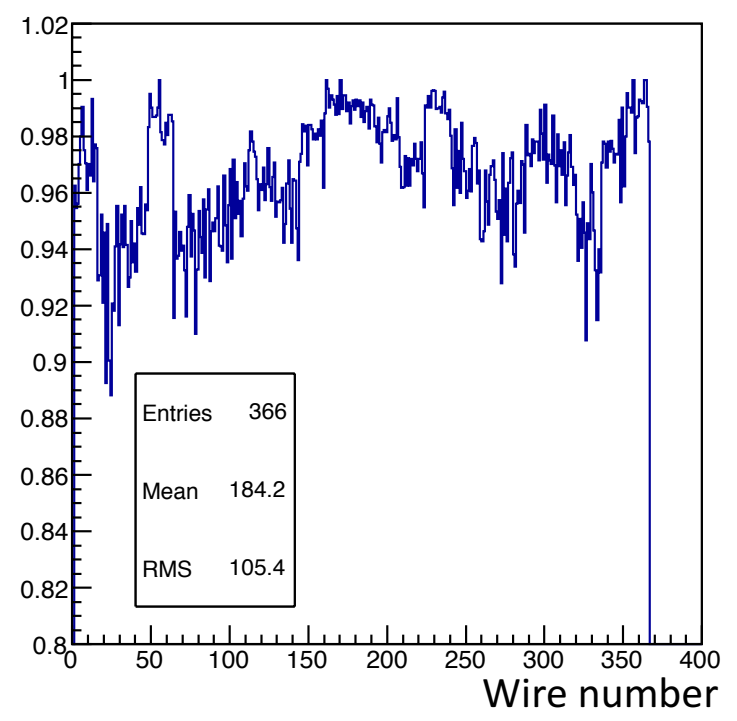

Right arm V2 efficiency

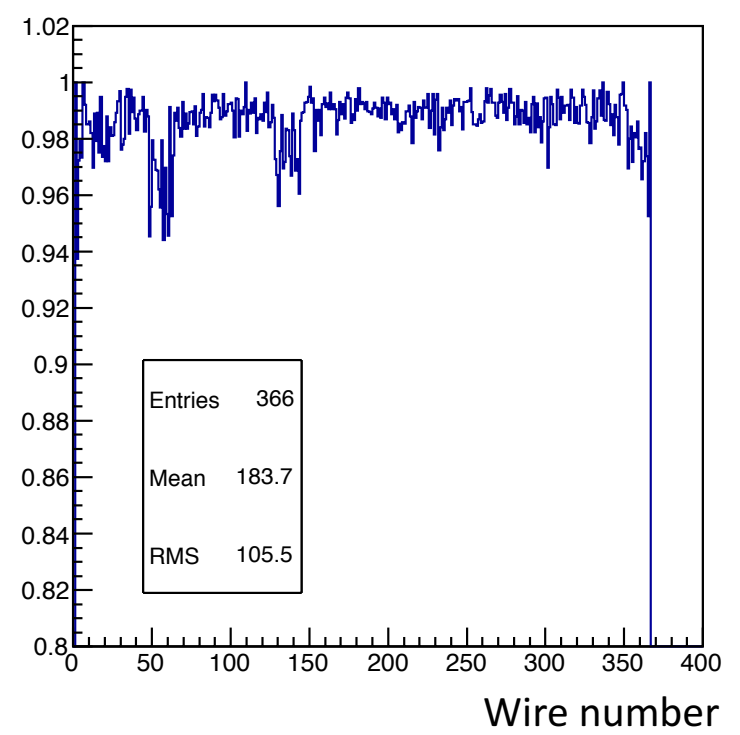

FIG. 5.4: The wire efficiencies for 4 VDCs planes of Right-arm HRS after 26 MAD cards with low efficiencies were replaced. 

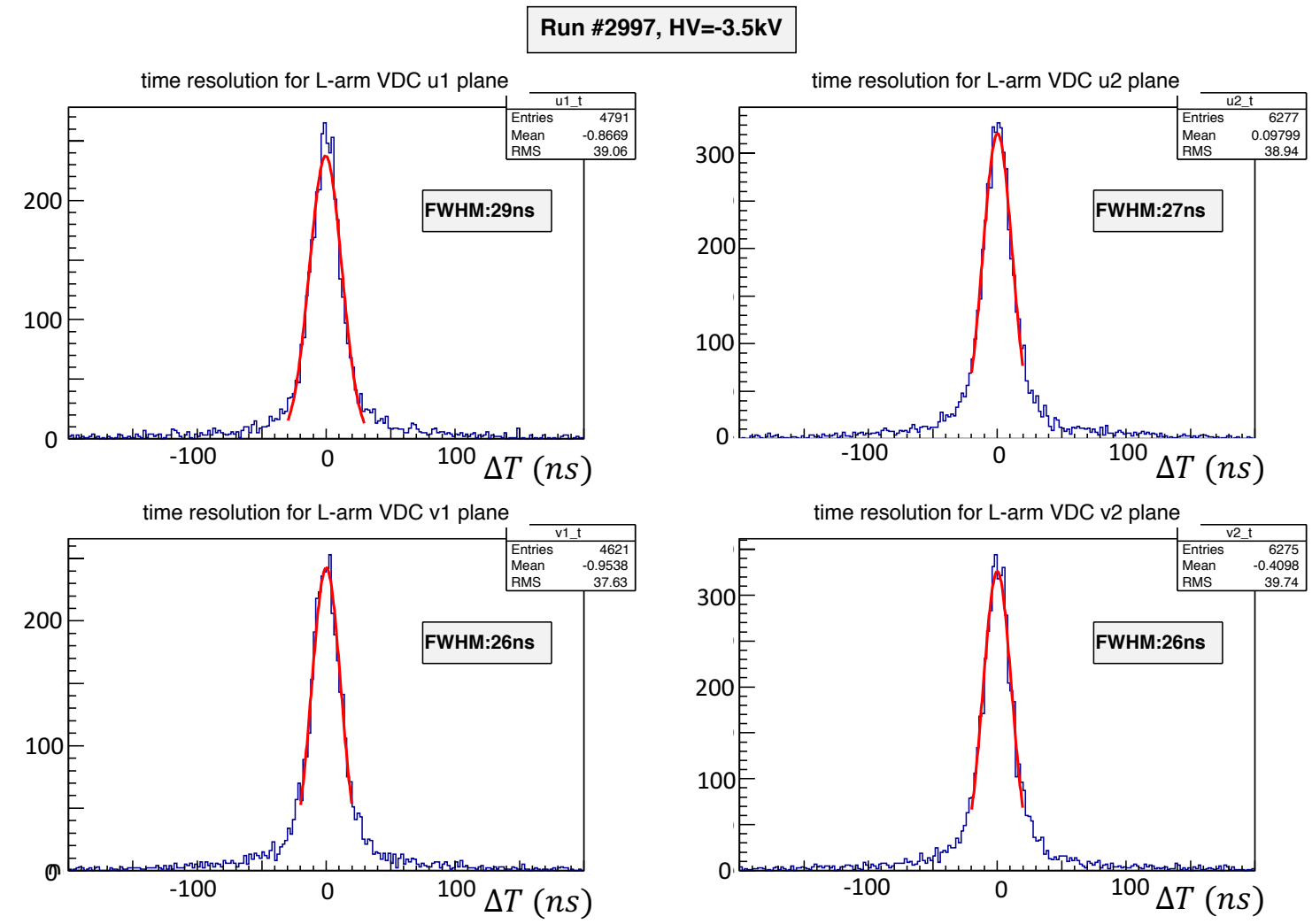

FIG. 5.5: Relative timing $\Delta T$ (see Eq. 5.2) for 4 planes of VDCs on the Right-arm HRS with HV being $-3.5 \mathrm{kV}$. 
given by

$$
\Delta t=\frac{1}{\sqrt{5}}\left(\frac{1}{\sqrt{4}} \mathrm{FWHM}\right)
$$

Then for HV being $-3.5 \mathrm{kV}$, the time resolution of the U1 plane is $\Delta t=\frac{1}{\sqrt{5}}\left(\frac{1}{\sqrt{4}} 29 \mathrm{~ns}\right)=$ 6.5ns. A HV scan with cosmic data was made at $-3.3 \mathrm{kV},-3.4 \mathrm{kV},-3.5 \mathrm{kV},-3.6 \mathrm{kV}$ and $-3.5 \mathrm{kV}$ was found to have the best time resolution.

\section{Level translator}

Previously, the LeCroy cards' output ECL signals were sent to LeCroy 1877 multi-hit Fastbus TDCs via 16-conductor, twisted-pair ribbon cables, however the outputs of the MAD cards are LVDC signals. In order to use older FastBus/VME TDC modules, a special LVDS-ECL level translator was developed and constructed by the JLab Electronics Group [72]. The translator accepts LVDS signals from a MAD card and then converts them to ECL signals, which are finally transmitted to 16 TDC channels via ribbon cable. Fig. 5.6 shows the level translator used for experiment E12-07-108.

Every MAD card needs one level translator to transmit the signals to the TDC and thus there are 23 level translators for each VDC wire plane. On each spectrometer arm, a level translator rack was installed near the TDCs. There was one fan on top of the rack and one fan at bottom of the rack to keep the translator cards cool. A new power supply for level translators was installed near the rack. Fig. 5.7 shows the level translator rack and power supply.

\subsubsection{Straw Chamber Improvement}

For experiment E12-07-108, one SC was installed on each HRS arm to improve the tracking accuracy of the multi-tracks events (see section 4.2.2). The SC was installed between the 


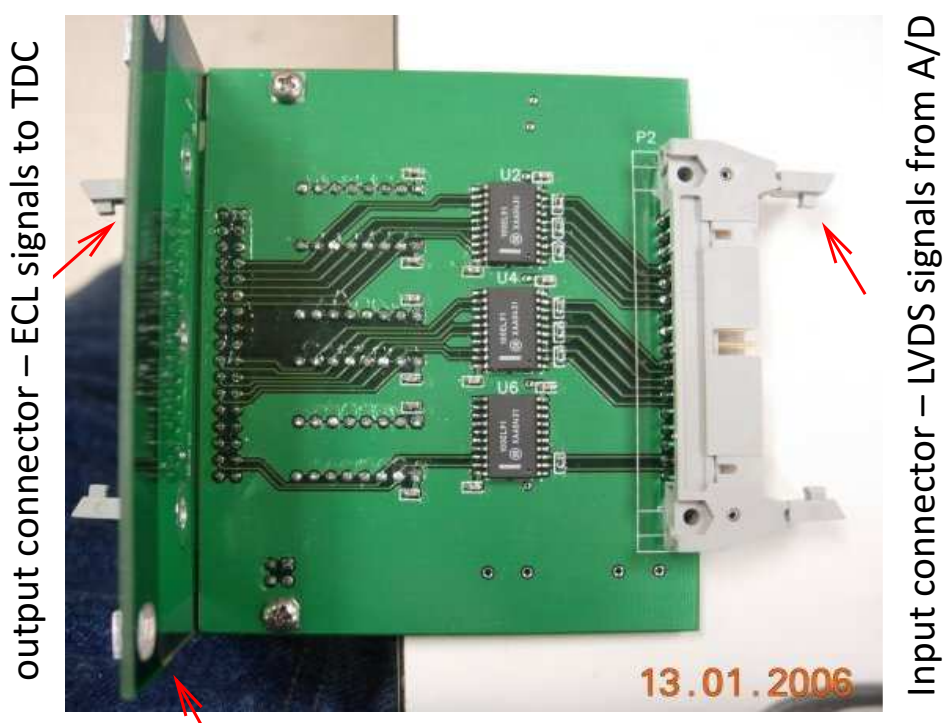

FIG. 5.6: Photograph of one level translator card designed for the MAD cards.
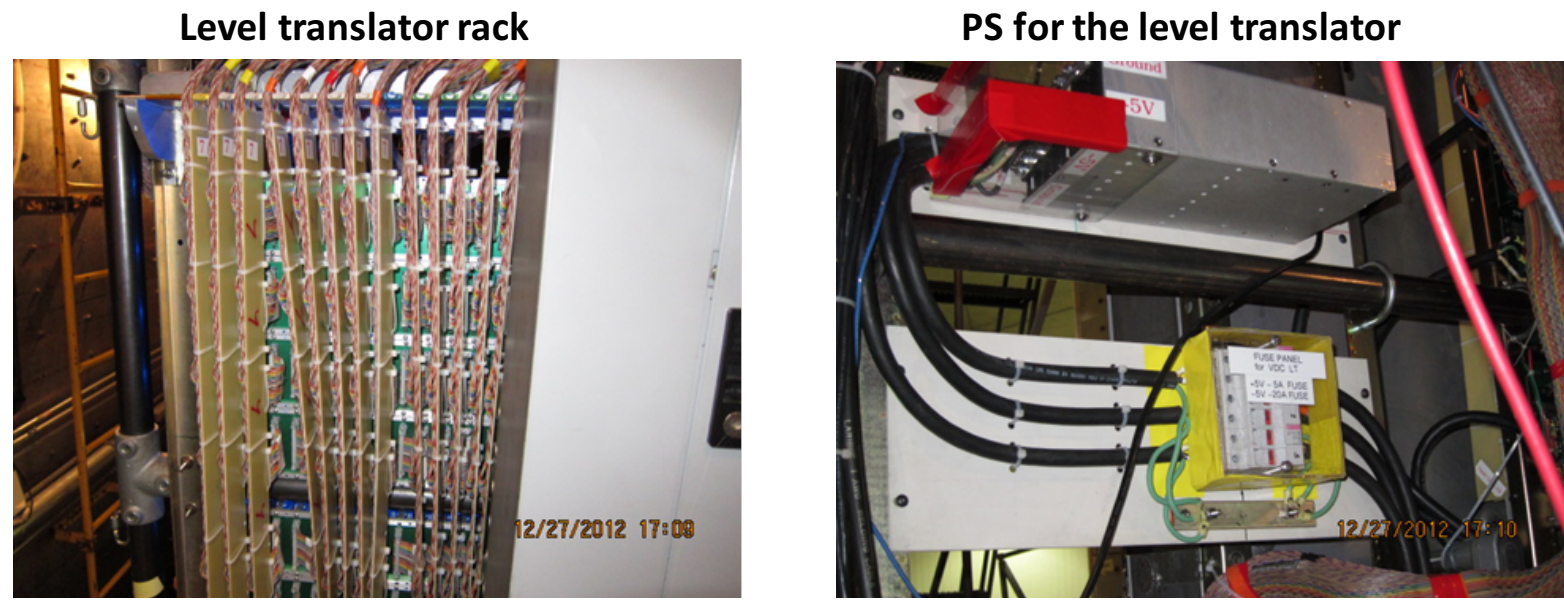

FIG. 5.7: Photograph of the level translator and its power supply. The left panel shows the level translator rack and the right panel shows the power supply. 
VDCs and the Cherenkov detector, and to fit the SC in the limited space between them, the frame of the SC was modified. Many modifications and improvements were done to make the SC operate: the gas distribution system was improved, new HV distribution boards were installed, low voltage power supplies and level shifter were installed, and numerous electronics problems were fixed. Fig. 5.8 shows one SC placed on the Hall A floor during modification before being installed.

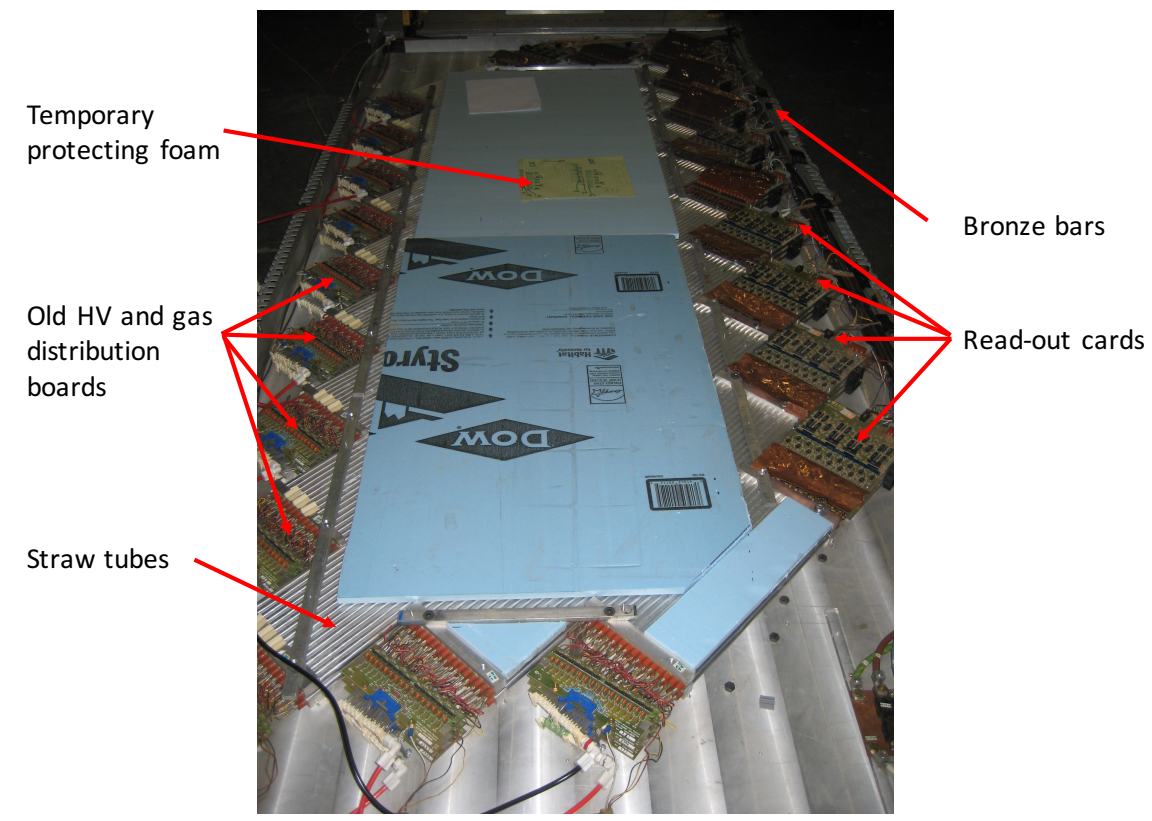

FIG. 5.8: Photograph of one SC during modification before experiment 12-07-108. Only three $\mathrm{U}$ planes of straw tubes are visible on this photograph.

When charged particles pass through the SC, an electron avalanche happens near the anode wire and a analog signal is produced at the wire. The analog signal is amplified and discriminated at the read-out card. The signals of a group of eight neighboring wires are then processed by the same multiplexing chip, which gives a logic pulse whose width depends on which wire is fired. The amount of electronics required for the SC is significantly reduced by multiplexing the signals. The $45 \mathrm{mV}$ signal produced by the multiplexing chip is converted to a $800 \mathrm{mV}$ signal in the level shifter and is sent to the multi-hit Fastbus 
TDCs via a 16-conductor, twisted-pair ribbon cable. The TDC records the arrival of the leading edge and the trailing edge of the logic signal. The time difference between the leading edge and the common stop given by the trigger gives the drift time. The diagram of the logic of the read-out system is shown in Fig. 5.9.

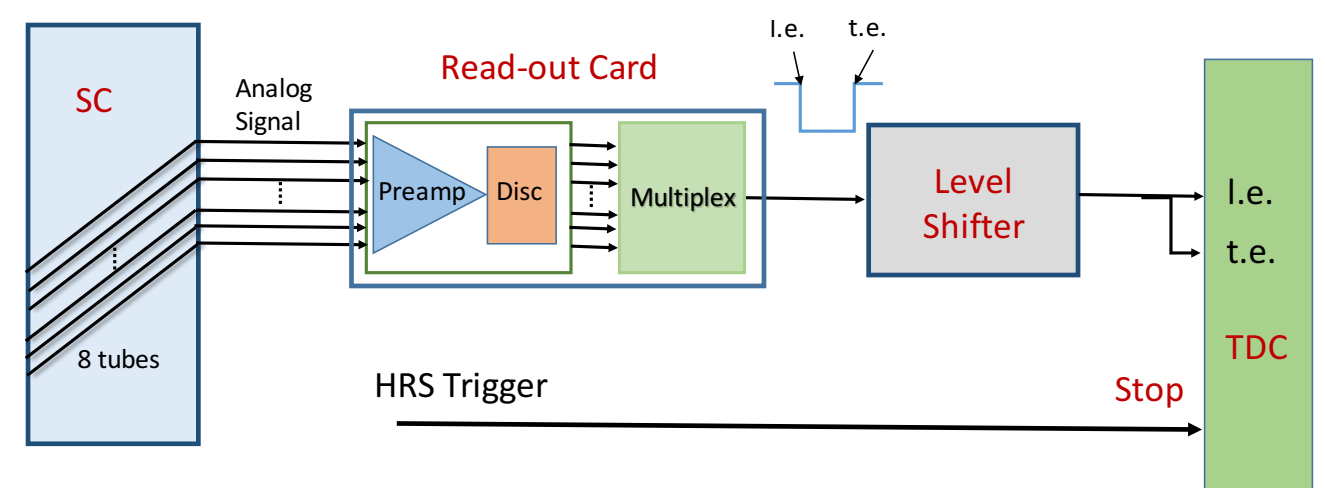

FIG. 5.9: Block diagram for the logic of the SC signal. (l.e. = leading edge, t.e. = trailing edge).

The tubes of SC are divided into 11 subgroups for $\mathrm{U}$ planes and 10 subgroups for V planes. Each subgroup consists of 48 tubes: there are three U planes of 16 tubes or three $\mathrm{V}$ planes of 16 tubes in each subgroup. The tubes in each subgroup share the same HV distribution board and the same gas input pipe. The three read-out cards of each subgroup are located together and share the same low voltage and threshold inputs.

\section{HV distribution improvement}

As shown in Fig. 5.8, the HV and gas distribution boards are at one end of the straw tubes and the read-out cards are at the other end of the tubes. Fig. 5.8 shows the old HV distribution boards: 3 layers of boards were installed together and each board was connected with 16 anode wires from one plane of three planes respectively.

During a test before experiment E12-07-108, it was observed that the HV often tripped because of a gas nununiform distribution. To resolve the problem, a new gas distribution 
system was designed and new gas distribution boards needed to be installed. The new gas distribution boards were as wide as one subgroup of tubes. However the old three layers of HV boards took all the space and none was left to install the new gas distributor boards. Thus, a new HV distribution board was designed, which was one board connecting with 48 wires, 3 layers of 16 wires of one subgroup of tubes (see Fig. 5.10). By replacing the old three layers of distribution boards with one board, a lot of space was saved. This made installing new gas distributor boards possible by placing the new HV board on top of the gas distribution board. With the new HV boards, all the HV wires of one subgroup are connected with one board and thus they are more well organized and much easier to access. When we find a bad tube and are not able to fix it, we disconnect both the wire to the HV board and the signal wire to the read-out card.

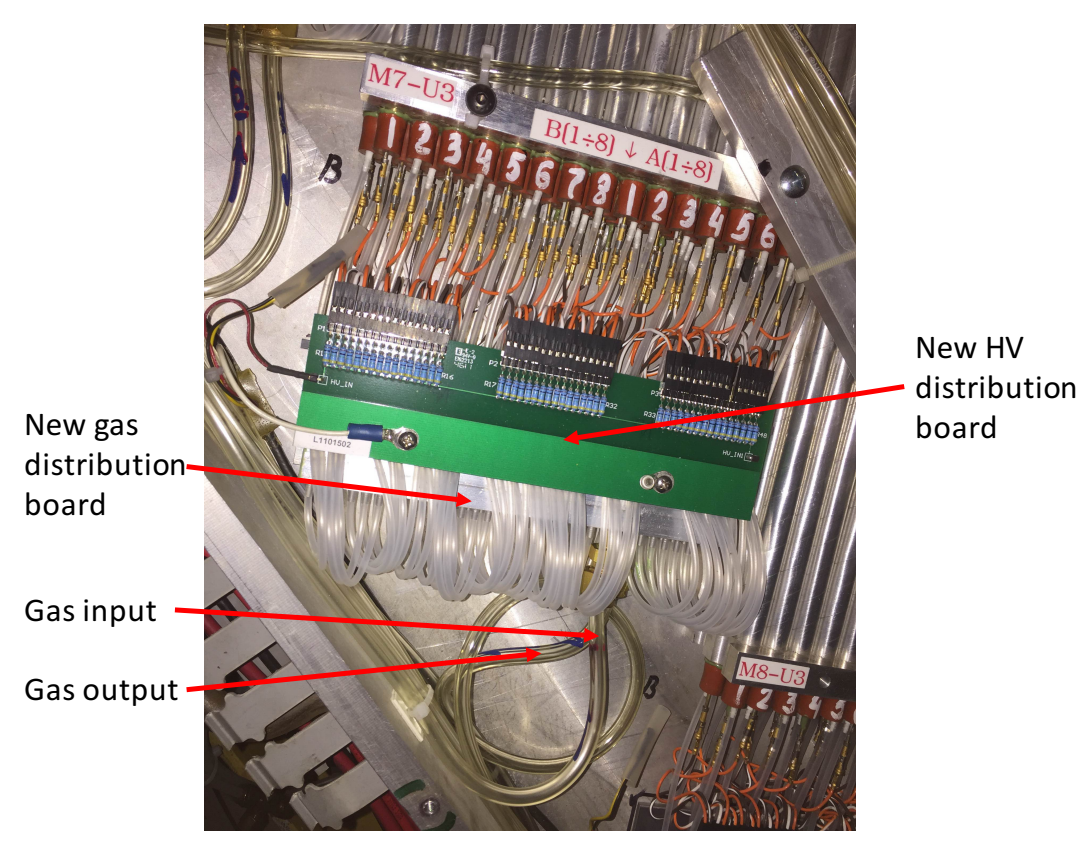

FIG. 5.10: Photograph of the new HV distribution board and new gas distribution boards. 


\section{Gas distribution system improvement}

The SC tubes are filled with gas mixture of $62 \% / 38 \%$ argon/ethane by volume. The gas input of the SC is monitored by one magnehelic gauge at the side of the SC (see Fig. 5.11). The input gas is transported in one transparent pipe and then is distributed to two pipes, one for the $\mathrm{U}$ planes and one for the $\mathrm{V}$ planes, controlled by the green blue valves in Fig. 5.11. Then the gas is distributed into 11 pipes for the $\mathrm{U}$ planes and 10 pipes for the V planes, each pipe for the gas input of one subgroup of 48 tubes (see section 5.1.2). The gas distributions to each subgroup of tubes are controlled by the small valves as shown in Fig. 5.11.

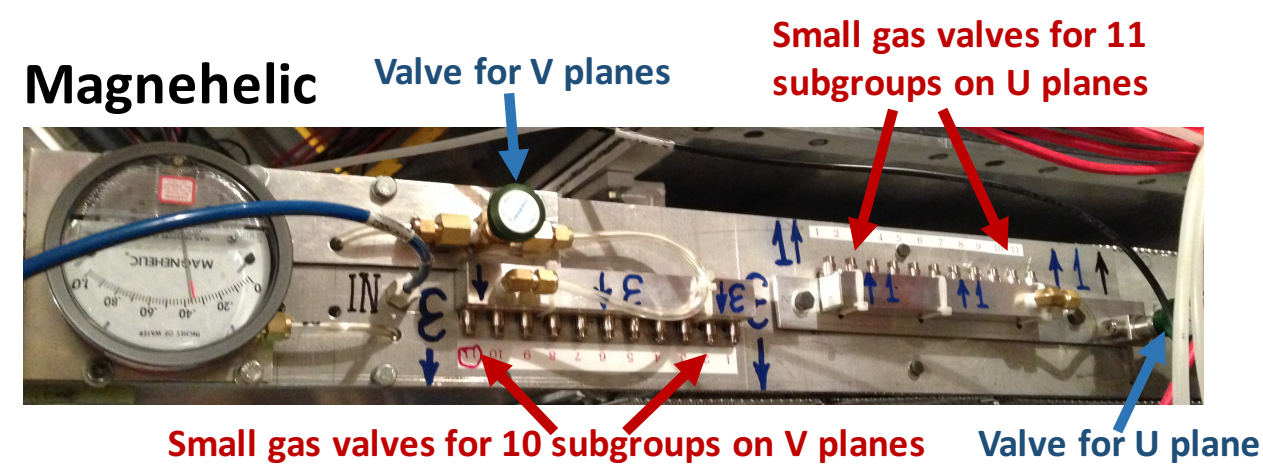

FIG. 5.11: Photograph of the magnehelic gauge and gas valves at the side of the SC. The input gas pressure is monitored by the gauge and are distributed to $\mathrm{U}$ planes and $\mathrm{V}$ planes. Then the gas is distributed to 11 pipes for the $\mathrm{U}$ planes and 10 pipes for the $\mathrm{V}$ planes, which are controlled by the small valves as shown.

For Experiment E12-07-108, the gas distribution system of each subgroups of tubes was redesigned and new gas distribution boards were installed (see Fig. 5.10). In the old system, the gas flows through the tubes one by one. For one subgroup, the gas flows into the first tube at one end and flows out the tube at the other end, where the second tube is connected with the first one with a gas pipe. The gas from the first tube flows into the second tube and then flows into the third tube in the same way. The left panel of Fig. 5.12 
shows the scheme of the old gas distribution system. The old system was straightforward and easy to achieve, but there was one big disadvantage. Because the long distance that the gas flowed through the tubes one by one, the gas pressure dropped dramatically after a couple of tubes and the decreased gas pressure caused frequent HV trips. To resolve this problem, a new gas distribution system was designed.

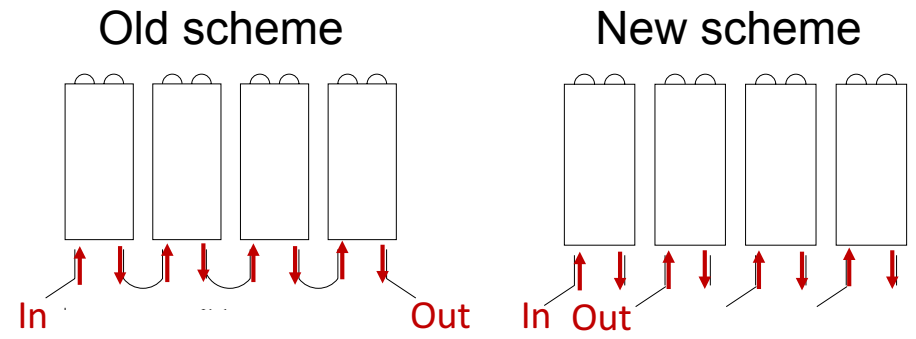

FIG. 5.12: Old and new gas distribution systems for each subgroup of tubes. The left panel shows the old one and the right panel shows the new one. The black lines represent the gas tubes.

In the new gas distribution system, the gas input for each subgroup of 48 tubes are distributed into 24 gas pipes by the new gas distributor board, each pipe for the gas input of two tubes. The gas flows into one tube from one end of the tube and flows out of the tube at the other end, where it flows into the second one (Fig. 5.12). By providing the gas input for the tubes at the same time instead of flowing the gas through the tubes one by one, the gas pressure imbalance problem is resolved. There are 24 gas outputs for each subgroup and they are collected into one pipe by the gas distributor board. Finally, these gas output pipes are located at one corner of the $\mathrm{SC}$ and the gas just flows out into the air (see Fig. 5.13).

These gas output pipes make it convenient to check whether there is leakage or blockage in one subgroup of SC tubes. First, one turns off most of the small valves and leaves just several open (see Fig. 5.11). Keeping the number of the open small valves the same and making sure the one corresponding to the subgroup of tubes that is to be tested is 


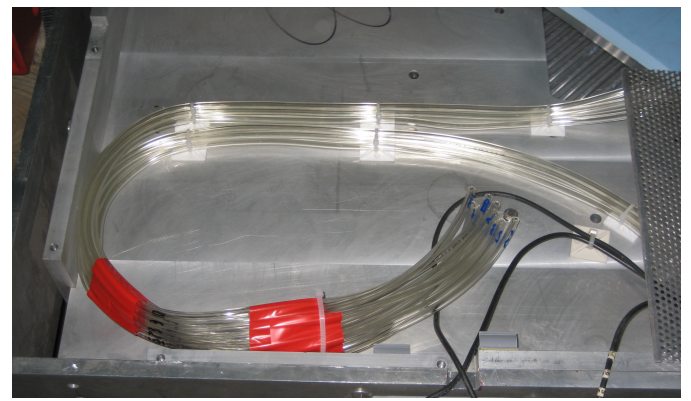

FIG. 5.13: Photograph of the gas output pipes for the SC of the U planes One pipe corresponds to one subgroup of tubes.

kept open. Then one person blocks the output gas pipe to the subgroup of tubes that you want to test. There are many ways to block the pipe, the easiest way is to block it with just a person's clean finger. The second person monitors the magnehelic gauge when the pipe is blocked and writes down the input gas pressure. Repeat this procedure for several pipes and compare the pressure readings. The input gas pressure should increase when the output pipe is blocked and the increased pressure should be close for different pipes. If there is leakage in one subgroup, the corresponding pressure should be lower than the rest. If there is blockage in one subgroup, the corresponding pressure should be the same when the output pipe is blocked. Now we see the reason to keep several small valves open. If many valves are left open, blocking one of the gas output pipes doesn't affect much and the increase in input gas pressure would be small and difficult to monitor. If just two valves are left open, blocking one of the pipes would cause the input gas pressure increasing above maximum range of the magnehelic gauge.

\section{Low voltage (LV) and threshold system improvement}

Two low voltage power supplies providing $+5.2 \mathrm{~V}$ and $-5.5 \mathrm{~V}$ nominal preamp voltages for all the read-out cards on the $\mathrm{U}$ and $\mathrm{V}$ planes of SC were installed on each HRS arm. Two 
threshold voltage power supplies was also installed on both spectrometer arms to provide the $+3.0 \mathrm{~V}$ nominal threshold voltage for the read-out cards.

The read-out cards are located at one side of the SC (see Fig. 5.8). Close to the readout cards, along the side of the SC frame, several bronze bars are installed. The bronze bars are connected with the low voltage and threshold power supplies. The read-out cards are connected to the bronze bars at corresponding points along the SC frame and thus get the low voltage and threshold voltage. During tests before the Experiment E12-07-108, unstable low voltage and threshold voltage were observed which caused missing signals of subgroups of tubes. Measuring at each read-out card, the voltages dropped as the distance between the read-out cards and the power supplies increased. The bronze bars were taken out and cut shorter to get rid of the unnecessary part, where there are no read-out cards close and connected. By deceasing the length of the bronze bars, the amount of voltage dropped along the SC frame decreased. After the shorter bars were installed, the read-out cards were observed to be much more stable.

\section{Signals testing}

After all the improvements and modifications were done to the SC, both the analog signals collected on the anode wires and the multiplexed digital signals sent to TDCs were carefully examined.

Placing one 55Fe radiation source above the tube to be examined, a signal would be produced on the corresponding anode wire. Then we connected the anode wire to a discriminator-amplifier and then sent the signal to an oscilloscope and observed the signal. Moving the 55Fe source above different tubes, the signals should have similar shapes and amplitudes. After making sure the tubes operate well and produce good analog signals, we sent the digital signals from the level shifters to the oscilloscope. The signals of 8 neighboring tubes are multiplexed to one channel, meaning when the oscilloscope 
is connected with one channel and 55Fe was moved across the 8 neighboring tubes, the oscilloscope should show digital signals with different widths as the radiation source moves. Table 5.1 lists the pulse widths of the multiplexed signals of the 8 neighboring tubes in one channel. Using the different width, we can know which wire fired.

TABLE 5.1: List of the pulse widths of the 8 multiplexed signals in one channel of SC.

\begin{tabular}{ccccccccc}
\hline \hline Wire Number & 1 & 2 & 3 & 4 & 5 & 6 & 7 & 8 \\
\hline Pulse Width (ns) & 25 & 45 & 35 & 55 & 90 & 65 & 100 & 75 \\
\hline
\end{tabular}

\subsection{GMP optics}

The optics matrix links the focal plane coordinates $\theta_{f p}, \phi_{f p}, x_{f p}$ and $y_{f p}$ to target coordinates $\theta_{t g}, \phi_{t g}, y_{t g}$ and $\delta$. The optics calibration is the process to determine the matrix elements.

Fig. 5.14 shows a electron passing a sieve slit hole after scattering at a multi-foil target. The position of the electron at the sieve plane, which is called the real position of the electron, is known by determining which hole the electron goes through and the position of the sieve. The position can also be calculated by the reconstructed target variables (see Eq. 4.9 and Eq. 4.10). The calculated position should match with the real position after the optics calibration.

For previous experiments in Hall A, the optics matrix was calibrated with elastic or quasi-elastic electron-carbon events with a $5 \mathrm{~mm}$ thick stainless steel sieve, which was thick enough to stop the electrons. As a result, the events from electrons passing through the sieve holes are easy to resolve and clean cuts selecting the electron events can be made. The GMp experiment was designed to measure the elastic $e-p$ cross sections in the high $\mathrm{Q}^{2}$ 


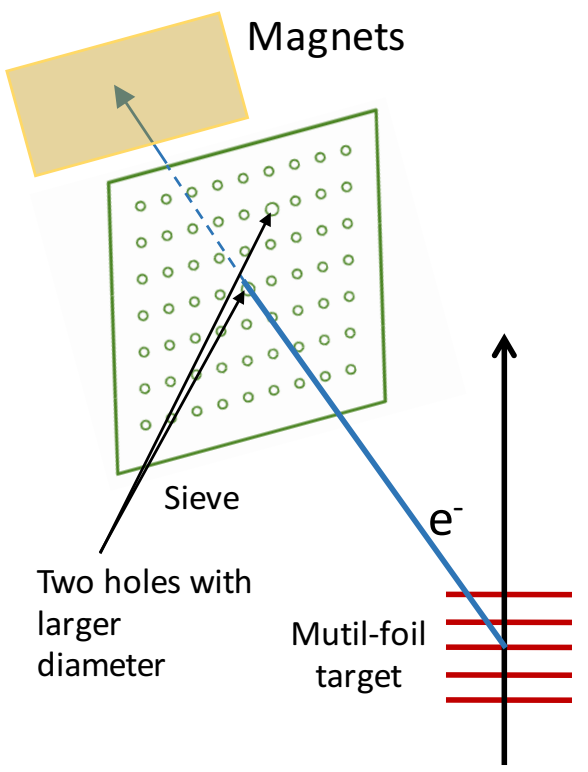

FIG. 5.14: Scheme shows a electron scattering at the multi-foil target goes through a sieve slit hole.

range from 7 to $11 \mathrm{GeV}^{2}$. In this $\mathrm{Q}^{2}$ range, optics calibration with elastic or quasi-elastic events would take too much time due to the small cross section in this energy range. Also, the GMp experiment was conducted with the DVCS experiment at the same time while DVCS experiment had the higher priority using the left-arm HRS. The right-arm HRS angle was limited to no less than $48^{\circ}$ due to the DVCS calorimeter's position. This large angle of the right-arm HRS also limited the rate we could get from the elastic or quasielastic events. As a result, it was decided to use inelastic $e-p$ events to conduct the optics calibration for the GMp experiment.

\subsubsection{New Sieve for GMp Optics}

In the case of using the inelastic scattering for the GMp optics calibration, the $5 \mathrm{~mm}$ thick stainless steel sieve is to thick to be useful. For the optics data, the HRS momentum is set 
to a value in the inelastic range which would provide a reasonable scattering rate. However, there are electrons scattered at the target with higher momentum than the HRS setting value and it is possible that their momentum after punching through the sieve is within the HRS momentum acceptance. These events would merge with the electrons passing through the holes and thus make the optics calibration inaccurate. Thus a thicker sieve made of tungsten was used for the GMp experiment to stop those electrons from punching through the sieve. Fig. 5.15 shows the reconstructed $\theta_{t g} v s . \phi_{t g}$, which are almost identical to the positions of the electrons at the sieve plane, with the old steel sieve and the new tungsten sieve.

The top panel of Fig. 5.15 is the plot of $\theta_{t g} v s . \phi_{t g}$ with the old steel sieve during the commissioning run in March 2014 and the bottom panel shows that with the new 1 inch thick tungsten sieve during the commissioning run in Dec. 2014. As shown in Fig. 5.15, there is much less background with the new sieve and this makes it possible to make a clean cut of the events passing through the sieve holes and then calibrate the optics matrix. The new sieve is drilled with 7 rows $\times 9$ columns of holes. The diameters of the holes are $4 \mathrm{~mm}$ and there are two holes with larger diameter $6 \mathrm{~mm}$. As shown in the bottom panel of Fig. 5.15, it is obvious to notice there are two holes with more electron events than the rest. These two holes are used to indicate which sieve holes the events are corresponded to. For example, there are 7 rows $\times 9$ columns of holes in the new sieve while only 6 rows and 5 columns at most can be "seen" due to the HRS acceptance. With the two large holes, it is easy to tell the column number and row number correlated with the events. The diagrams of the new tungsten sieve are included in Appendix A. 


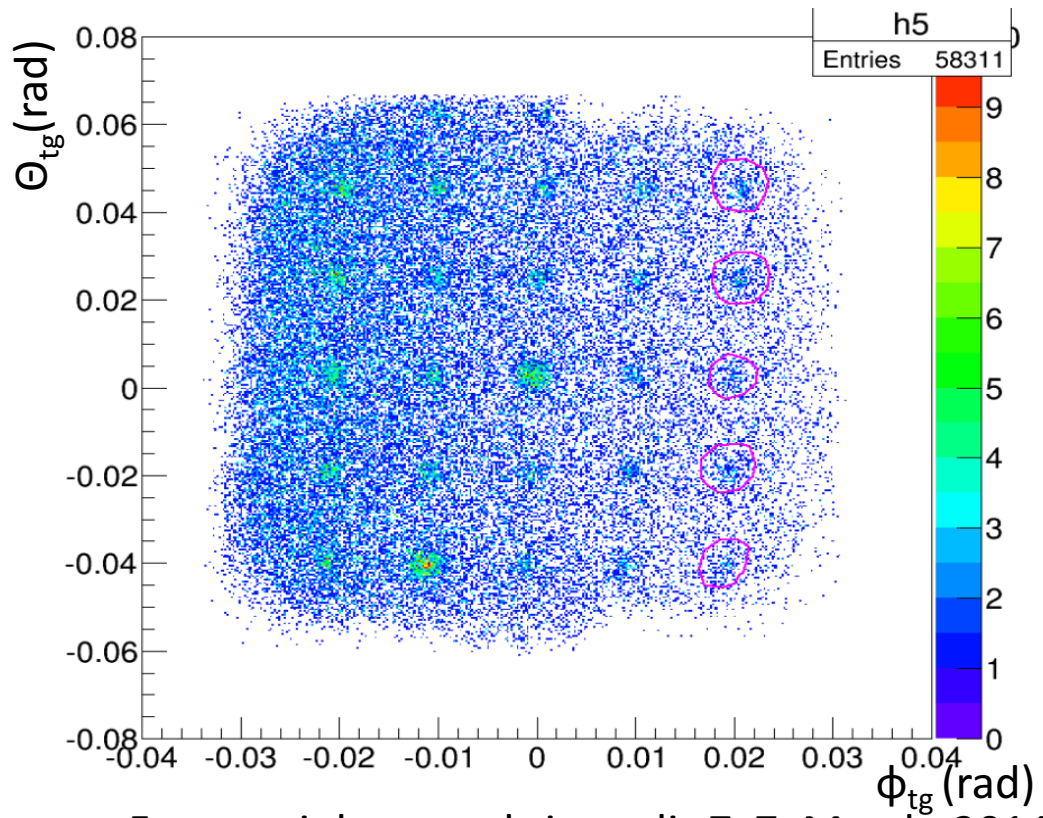

$5 \mathrm{~mm}$ stainless steel sieve slit:7x7. March, 2014

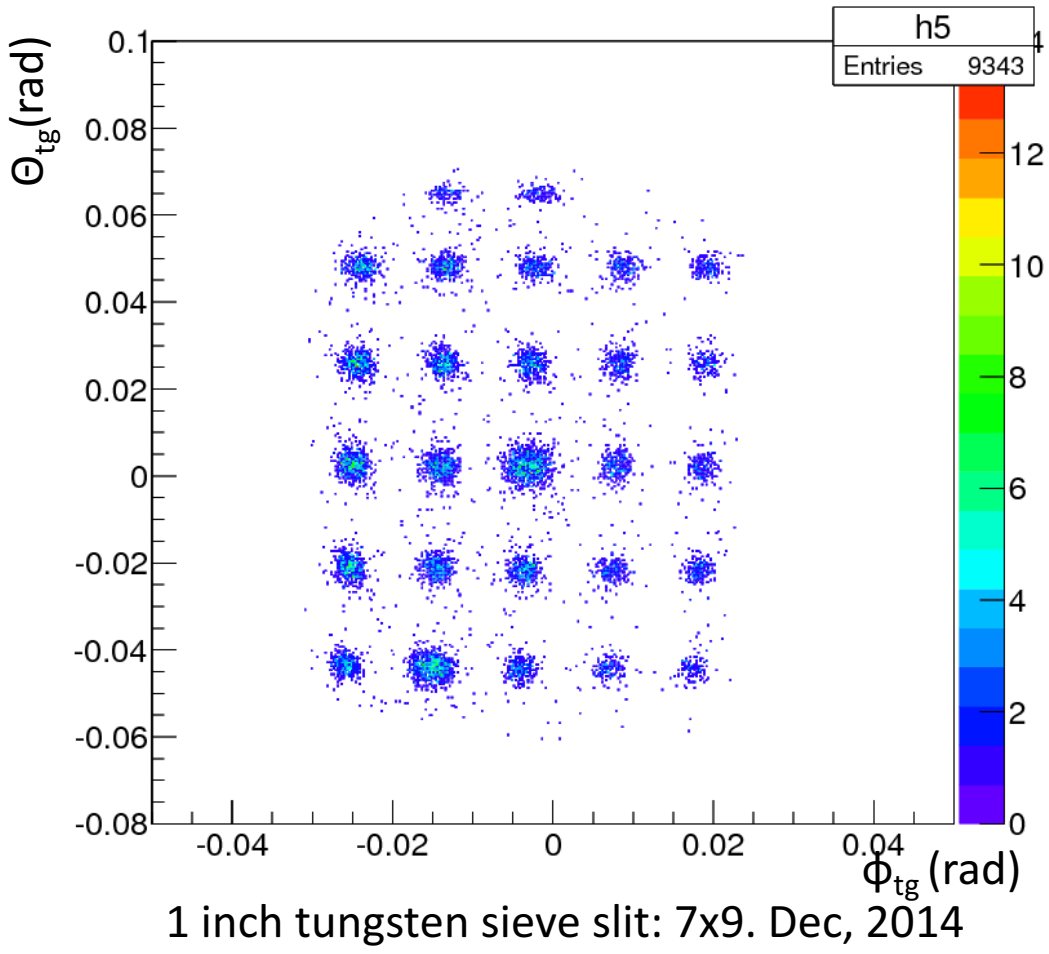

FIG. 5.15: $\theta_{t g}$ vs $\phi_{t g}$ with different sieves. The data of the top panel was taken with a $5 \mathrm{~mm}$ steel sieve in March 2014 and the data of the bottom panel was taken with a 1 inch tungsten sieve in December 2014. 


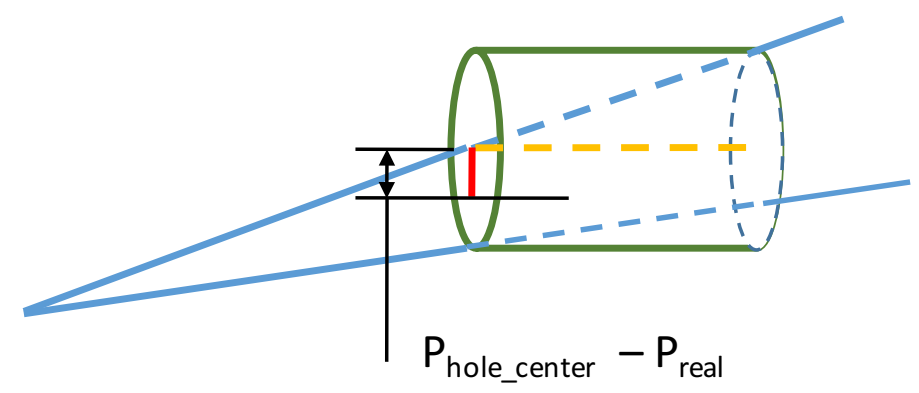

FIG. 5.16: Scheme showing the offset from the hole center to the average position distributions caused by the thickness of the sieve.

\subsection{2 $\theta_{t g}$ and $\phi_{t g}$ Optimization}

For this thesis, the optics data taken in the spring of 2015 were used for RHRS. During this time, Q1 was replaced with the SOS quadrupole because of the failure of Q1. The radius of the SOS quadrupole is $12.5 \mathrm{~cm}$ while that of Q1 is $15 \mathrm{~cm}$, thus the SOS quadrupole has a smaller acceptance than Q1. For the LHRS, optics data for the spring of 2016 were used for this thesis. At that time, LHRS was still using Q1.

The $\theta_{t g}$ and $\phi_{t g}$ are optimized by matching the reconstructed positions with the real positions at the sieve plane through minimizing $\chi^{2}$ (see Section 4.3.2). The exact real position can not be obtained with just the sieve position information. However the distribution of the positions of the electron events that pass each sieve hole is symmetric and the average of the position distribution could be used as the real position. When the sieve was just $5 \mathrm{~mm}$ thick, the center position of each sieve hole can be used as the real postion. But when the new 1 inch thick sieve was used for the GMp optics calibration, the offset from the hole center to the average position distribution caused by the thickness of the sieve could not be neglected. Fig. 5.16 shows the scheme of the average position distribution offset to the hole center.

For the GMp experiment, the offset of each sieve hole was calculated and included in 
the optics calibration. The offset of each hole is listed in Table 5.2 and 5.3. The horizontal offsets of each column of sieve holes and the vertical offsets of each row of sieve holes for each foil target are the same. As expected, the offset increases as the hole gets farther away from the central hole of the sieve. For the central hole, the offset is not zero because the sieve was not perfectly located at the center of the HRS.

TABLE 5.2: List of the horizontal offset of each sieve hole for each foil target.

\begin{tabular}{c|c|c|c|c|c|c|c|c|c}
\hline \hline X_offset $(\mathrm{mm})$ & Col 1 & Col 2 & Col 3 & Col 4 & Col 5 & Col 6 & Col 7 & Col 8 & Col 9 \\
\hline \hline Foil target 1 & 0.70 & 0.56 & 0.42 & 0.26 & -0.02 & -0.30 & -0.44 & -0.58 & -0.72 \\
Foil target 2 & 0.72 & 0.57 & 0.42 & 0.27 & -0.02 & -0.30 & -0.45 & -0.59 & -0.74 \\
Foil target 3 & 0.73 & 0.58 & 0.43 & 0.27 & -0.02 & -0.31 & -0.46 & -0.61 & -0.76 \\
Foil target 4 & 0.75 & 0.60 & 0.44 & 0.28 & -0.03 & -0.32 & -0.47 & -0.62 & -0.78 \\
Foil target 5 & 0.77 & 0.61 & 0.46 & 0.29 & -0.03 & -0.33 & -0.48 & -0.64 & -0.79 \\
\hline
\end{tabular}

TABLE 5.3: List of the vertical offset of each sieve hole for each foil target.

\begin{tabular}{c|c|c|c|c|c|c|c}
\hline \hline Y_offset $(\mathrm{mm})$ & Row 1 & Row 2 & Row 3 & Row 4 & Row 5 & Row 6 & Row 7 \\
\hline \hline Foil target 1 & -1.13 & -0.84 & -0.55 & 0.02 & 0.58 & 0.87 & 1.15 \\
Foil target 2 & -1.15 & -0.86 & -0.56 & 0.02 & 0.59 & 0.89 & 1.18 \\
Foil target 3 & -1.18 & -0.88 & -0.57 & 0.02 & 0.61 & 0.91 & 1.20 \\
Foil target 4 & -1.21 & -0.90 & -0.58 & 0.03 & 0.63 & 0.93 & 1.23 \\
Foil target 5 & -1.24 & -0.92 & -0.60 & 0.03 & 0.64 & 0.95 & 1.26 \\
\hline
\end{tabular}

Fig. 5.17 shows the reconstructed positions at the sieve plane for the five foil targets after the $\theta_{t g}$ and $\phi_{t g}$ optimizations. The crosses in the figure are the positions of the center of the sieve holes including the position distribution offsets mentioned above. As shown in Fig. 5.17, the crosses and the reconstructed positions matched well. However, this estimation is only qualitative. To get more quantitative information about the accuracy of the optimization, the position distributions were projected on to the horizontal and 

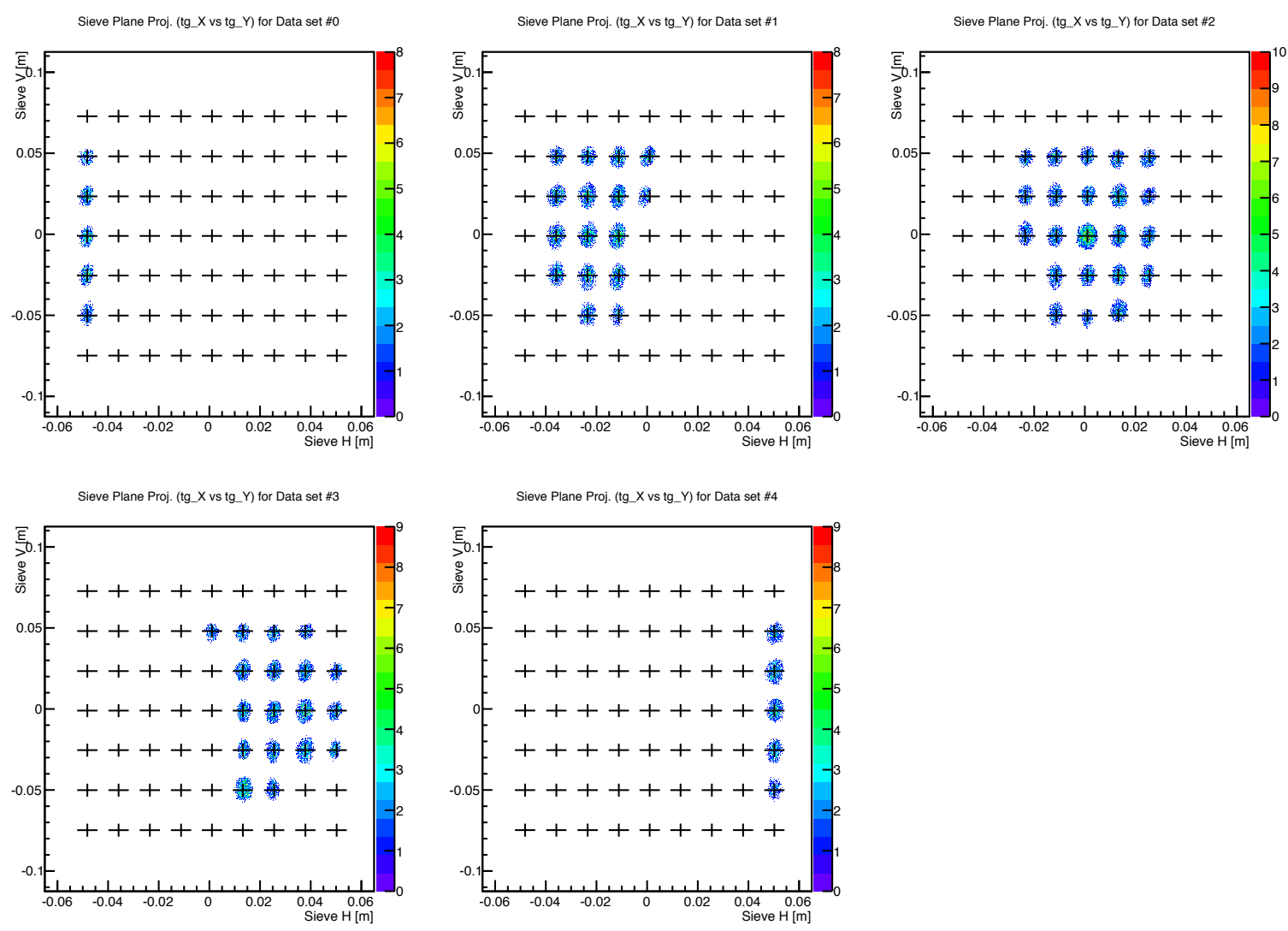

FIG. 5.17: The reconstructed positions at the sieve plane after the $\theta_{t g}$ and $\phi_{t g}$ optimizations. The crosses are positions of the center of the sieve holes with the distribution offset corrections. 
vertical directions. In each direction, a Gaussian fit was made for the events of each sieve hole respectively and the mean value of the Gaussian fit was compared to the corrected hole center position. The discrepancy in the horizontal direction indicated the accuracy of the $\phi_{t g}$ optimization and that in the vertical direction indicated the accuracy of the $\theta_{t g}$ optimization. Fig. 5.18 shows the position distributions in the horizontal direction with the Gaussian fits of the middle row of the sieve holes for the middle foil target.

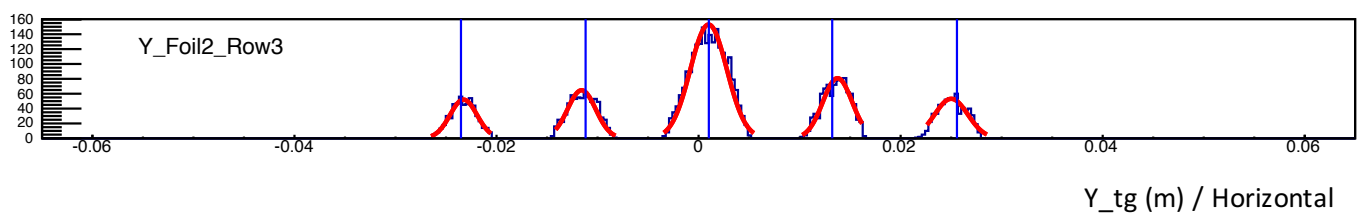

FIG. 5.18: The position distribution in the horizontal direction with the Gaussian fittings of the middle row of the sieve holes for the middle foil target. The blue lines indicate the hole center positions with corrections.

The average of the absolute discrepancy is $0.39 \mathrm{~mm}$ in the horizontal direction and $0.78 \mathrm{~mm}$ in the vertical direction. This doesn't meet the GMp accuracy requirement, which requires $0.18 \mathrm{mrad}$ uncertainty in the horizontal direction and $0.35 \mathrm{mrad}$ uncertainty in the vertical direction to get the cross section with $2 \%$ uncertainty. The sieve was about 1 $\mathrm{m}$ away from the target, thus $1 \mathrm{mrad}$ corresponds to about $1 \mathrm{~mm}$. Rechecking Fig. 5.17, it is easy to notice that even though there were 7 rows $\times 9$ columns of holes in the sieve, only limited holes were "seen" due to the acceptance of the HRS. And there is only one column of sieve holes "seen" for the most upstream and most downstream foil targets. With this limited number of data points, one sieve hole as one data point, the optics optimization couldn't reach the required accuracy.

To optimize $\theta_{t g}$ and $\phi_{t g}$ with better accuracy in the 2016 runs (not part of this thesis), more columns and rows and more holes between the existing holes were drilled in the sieve 
plate to obtain more data points.

\section{Optics Improvement with Pseudo MC data}

The Monte Carlo simulation program used by experiment E12-07-108 is talked about in Chap. 6.8 and the acceptance plots of the comparison between the MC and the experimental data taken in the spring of 2015 are presented in Chap. 7.1. The plots of kinematics $a$ (RHRS) show good agreement while discrepancies are observed for kinematics $b, c$ and $d$ (LHRS). We believe this is because the HRS optics and MC optics do not agree well.

As introduced in Chap. 3.2.2, the magnet configuration is of a QQDQ type on both arms of the HRS. In the spring of 2015, the first quadrupole (Q1) was not used on RHRS because of its abnormal behavior. It was replaced with the quadrupole from the Short Orbit Spectrometer (SOS) located in Hall C. Later in fall of 2016, the SOS quadrupole took the place of Q1 on LHRS too. A preliminary analysis shows that the comparisons between the $\mathrm{MC}$ and the experimental data with one-pass beam energy in the fall of 2016 are in good agreement for both LHRS and RHRS. We conclude the MC and the experimental data match well when the SOS quadrupoles are used while a discrepancy exists with Q1 kept. From 2015 and 2016, another improvement is that the HRS optics matrix was better calibrated with more holes drilled in the sieve plate.

The radius of the SOS quadrupole is $12.5 \mathrm{~cm}$ while that of Q1 is $15 \mathrm{~cm}$, thus the SOS quadrupole has a smaller acceptance than Q1. The HRS optics reconstructs events with worse accuracy near the edge of the acceptance, because the closer to the edge, the fewer events could be collected to perform the optics calibration. As a result, the events detected within the acceptance of SOS quadrupole were reconstructed overall more accurately than that of Q1. Besides, the optics were better calibrated in 2016 than 2015 due to a new sieve used, in which more holes were drilled and thus bigger acceptance were covered and more data points were collected for calibration. As a result, the MC and the experimental data 
match better with the SOS quadrupole.

To obtain better optics calibration for the data of the spring of 2015, a set of pseudo data produced using the $\mathrm{MC}$ near the edge of the acceptance was combined with the experimental optics data for calibration. Fig. 5.19 shows the calibration results with the pseudo data from the MC added, which were in red boxes.
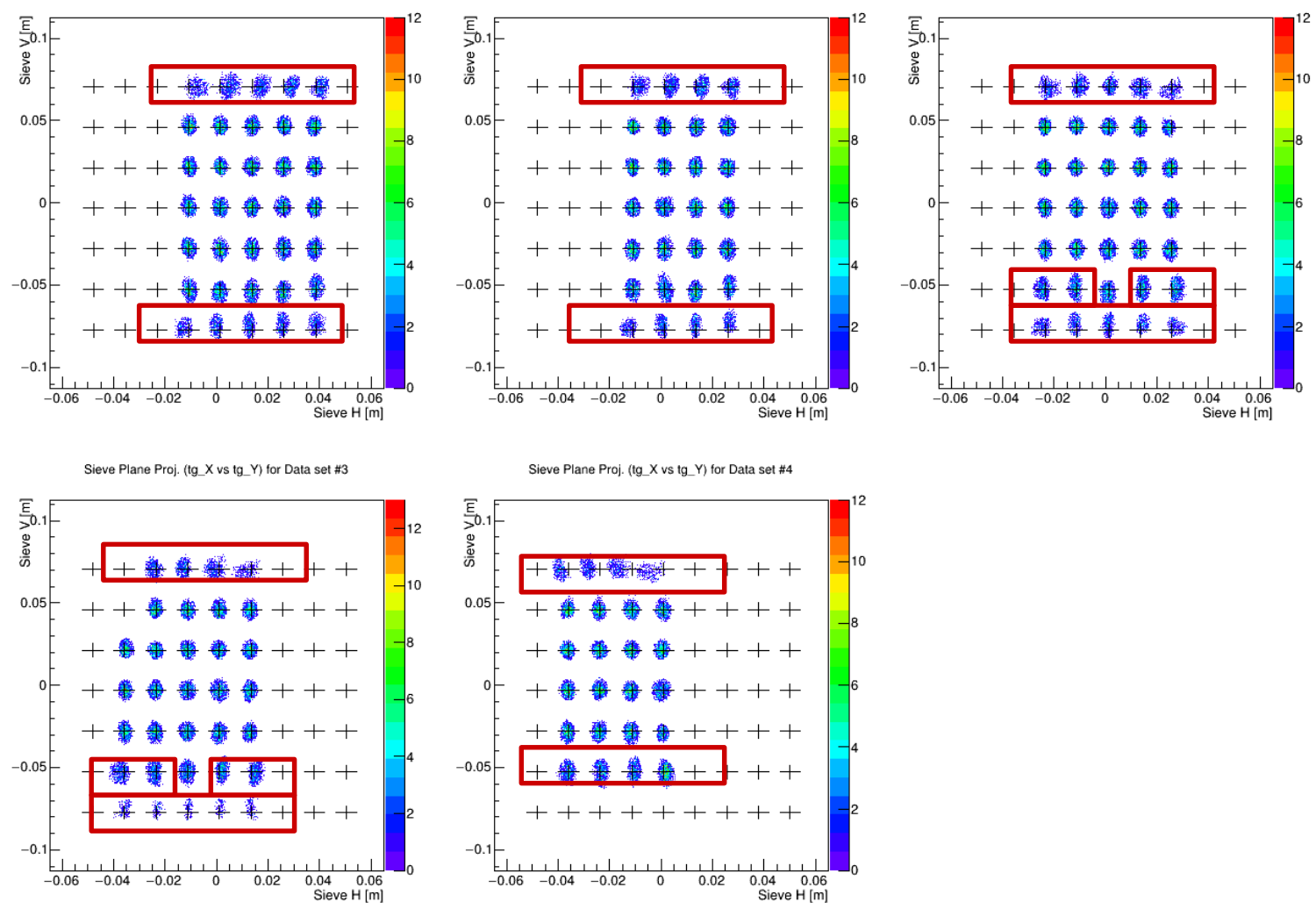

FIG. 5.19: The reconstructed positions at the sieve plane after the $\theta_{t g}$ and $\phi_{t g}$ optimizations with pseudo data produced by the MC are included. The crosses are the positions of the center of the sieve holes with the distribution offset corrections. The data points in red boxes are the pseudo data.

Fig. 5.20 gives the comparison between the $\mathrm{MC}$ and experimental data of kinematics $b$ (see Table 6.1)before and after the pseudo data added. A big improvement in agreement is observed mainly in the out-of-plane angle $\theta_{t g}$, in which direction most of the pseudo data were added. With the pseudo data from the MC near the edge of the acceptance 
added, it constrains the fit of the matrix elements in the optics calibration to prevent the fit "blowing up" when lacking data points. The big improvements are also observed for all other kinematics on LHRS arm in the spring of 2015, $c$ and $d$, where Q1 was used. However, the discrepancies still exist as shown in Fig. 5.20.
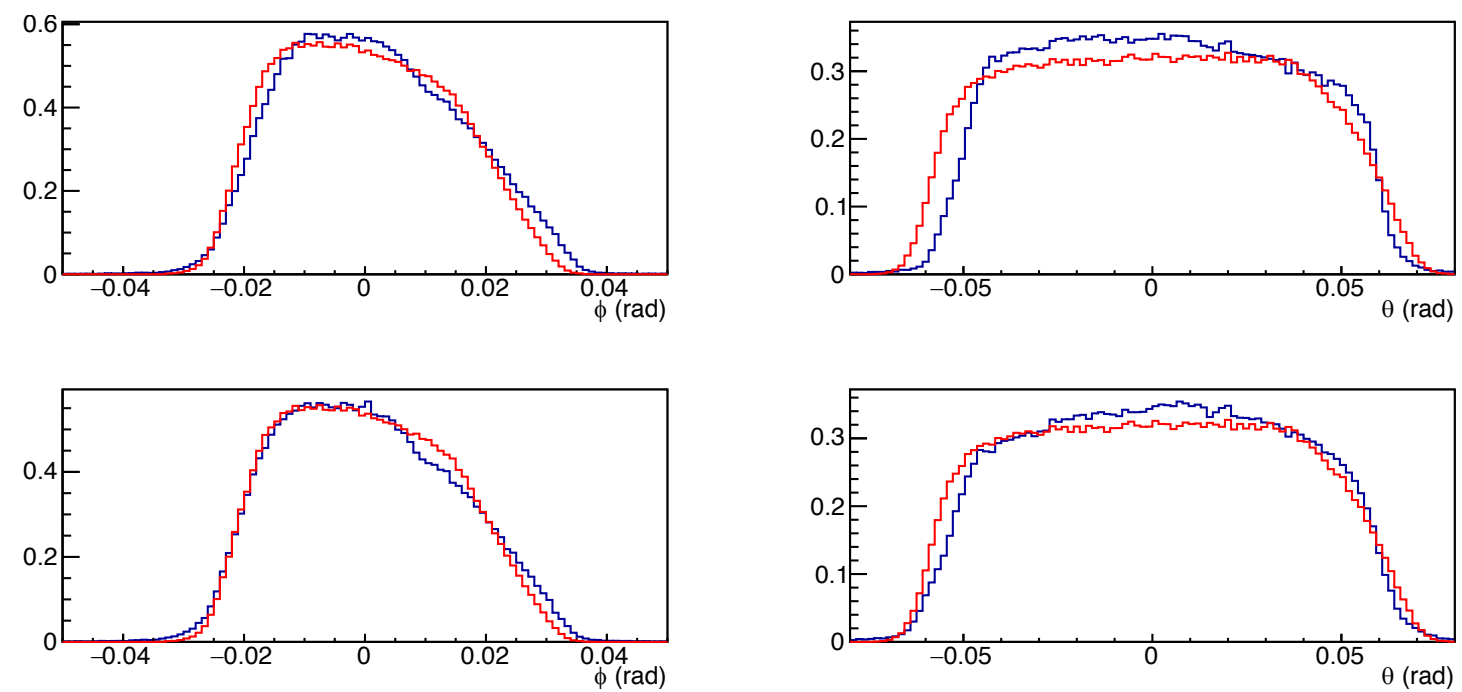

FIG. 5.20: The comparison of $\theta_{t g}$ and $\phi_{t g}$ between the MC and experimental data of kinematics $b$ (see Table 6.1). The top (bottom) two plots show the comparison before (after) the pseudo data from $\mathrm{MC}$ added in optics calibration.

Another Monte Carlo simulation has been performed by students Dien Nyugen and Thir Gautum using a carbon foil target instead of the liquid hydrogen target. The simulation was done in the deep-inelastic region, where the $e-p$ cross section almost doesn't change within the HRS acceptance. As a result, the distributions of the variables at the focal plane were determined by the acceptance of the spectrometer and the $e-p$ cross section doesn't contribute much. This allows us to investigate the MC forward and backward optics and the investigation indicates the MC optics needs to be corrected because the $\mathrm{MC}$ and data variables at the focal plane couldn't be matched very well. 


\subsection{3 $y_{t g}$ Optimization}

The $y_{t g}$ is related to the foil target positions along the $z$ direction in the hall coordinate system and thus it is optimized by matching the reconstructed foil target positions with the real foil target positions. The carbon foil targets were $3.75 \mathrm{~cm}$ apart and there were five of them. Fig. 5.21 shows the optimization results of the runs in April 2015, which compared the Gaussian fitting mean value of the reconstructed foil target positions with the real ones.
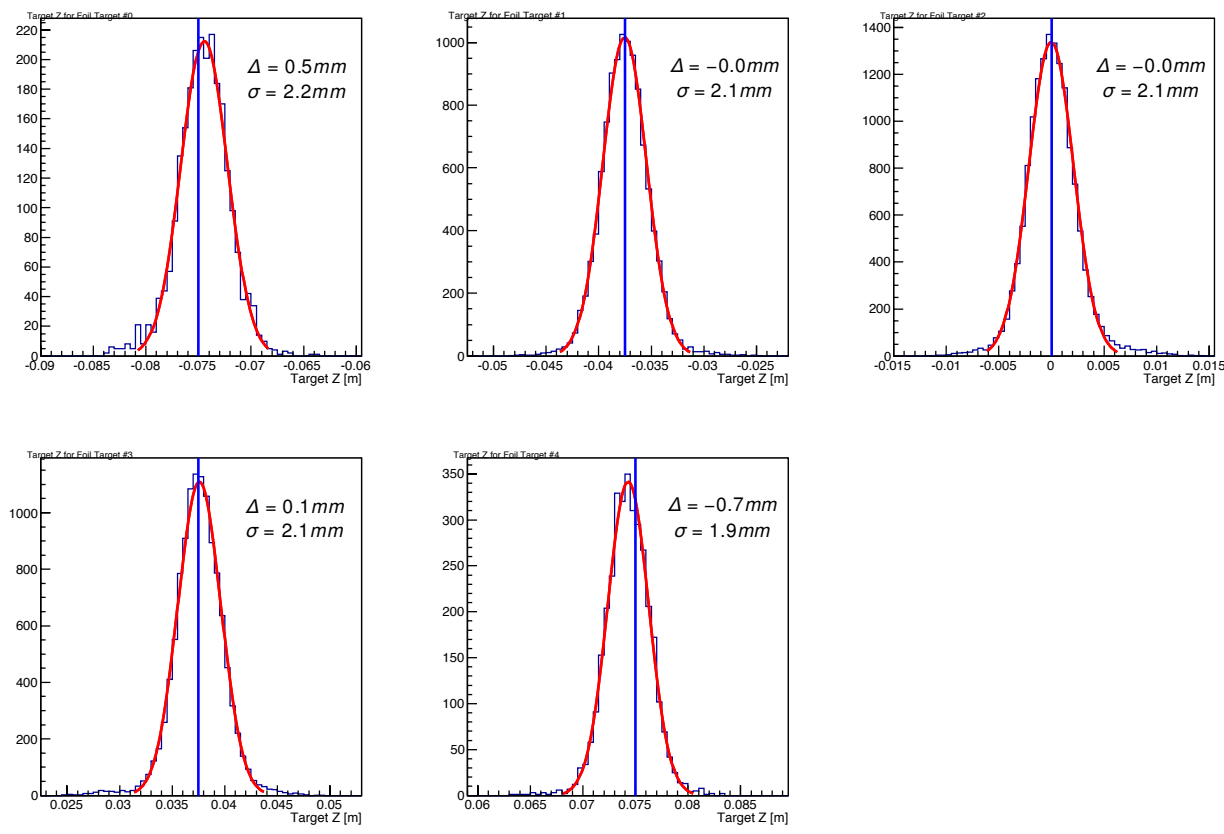

FIG. 5.21: The $y_{t g}$ optimization results which shows the Gaussian fitting mean values of the reconstructed foil target positions compared with the real ones. The blue lines indicate the real foil target positions. From left to right, top to bottom, the foil targets are from downstream to upstream. 


\subsection{4 $\frac{d P}{P}(\delta)$ Optimization}

$\frac{d P}{P}(\delta)$ is defined in Eq. 4.3. To calibrate the matrix elements for $\frac{d P}{P}$, measurements of five runs with $\frac{d P}{P}$ values varying from $-4 \%$ to $4 \%$ in steps of $2 \%$ were performed. Previously, the carbon foil targets combined with the sieve were used for $\frac{d P}{P}$ calibration. However, due to the small cross section for elastic scattering of the GMp experiment, a liquid hydrogen target was used instead.

In the previous experiments which conducted the $\frac{d P}{P}$ optimization with the carbon foil targets and sieve, the targets positions $z, x_{\text {beam }}$ and $y_{\text {beam }}$ (in hall coordinate system) provided the reaction vertex. With the reaction vertex, the scattering angle was then calculated by the position of the sieve hole through which the electrons passed. With the beam energy, scattering angle, and energy loss in the materials, the momentum of the electrons could be calculated at the entrance of the HRS.

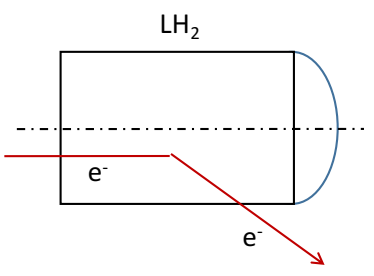

(1)

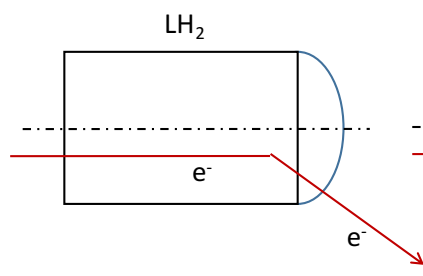

(2)

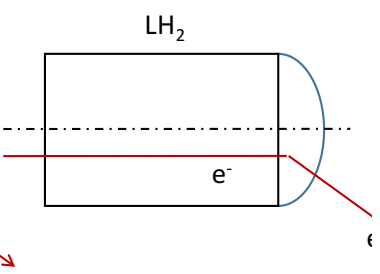

(3)

FIG. 5.22: Three cases for the electron scattering in the liquid hydrogen target. (1) Both the reaction vertex and the interaction point with the shell are in the cylinder; (2) the reaction vertex is in the cylinder and the interaction point with the shell is in the tip; (3) both are in the tip.

For the GMp experiment, the liquid hydrogen target was used instead of the foil targets. The horizontal beam position $x_{\text {beam }}$ and the reconstructed scattering angle $\phi_{t g}$ were used to calculate the reaction vertex (Eq. 4.8). The liquid hydrogen target is a $15 \mathrm{~cm}$ long cylinder with 1.5 inches radius ending with a tip with the same radius. The thicknesses of the aluminum shells of the cylinder and the tip are different. Thus the 
energy loss before and after the reaction vertex needs to be calculated carefully. Three cases were considered: both the reaction vertex and the interaction point with the shell are in the cylinder, one is in the cylinder and the other is in the tip, both are in the tip. These three cases are shown in Fig. 5.22
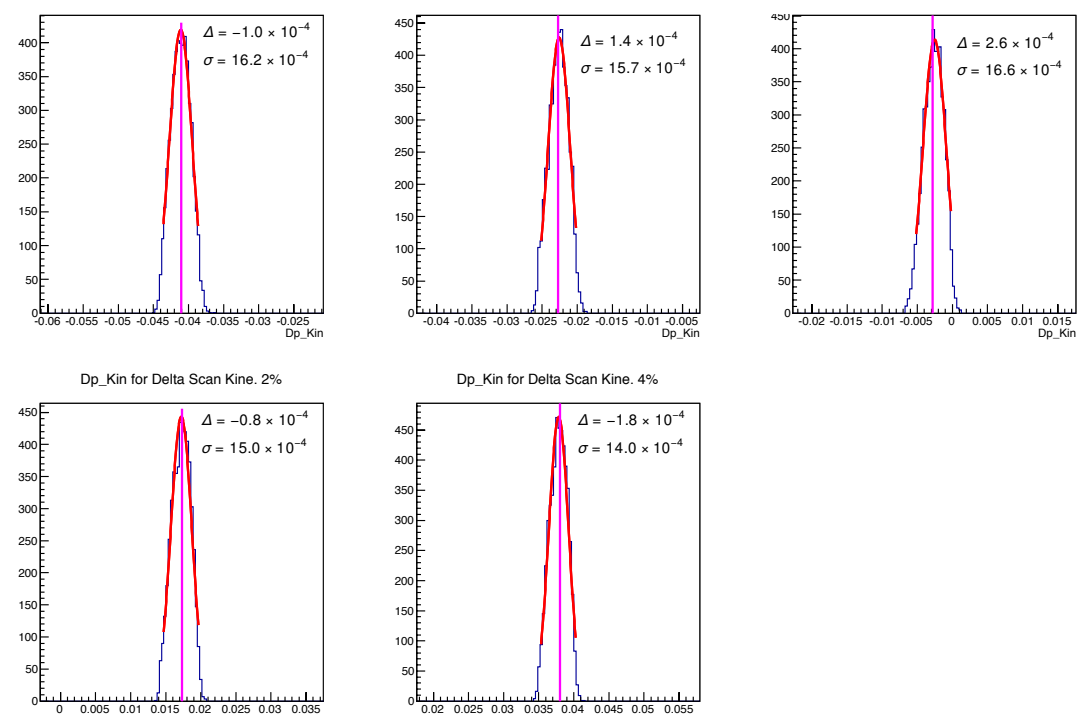

FIG. 5.23: The $\frac{d P}{P}$ optimization results (see text). From left to right, top to bottom, $\frac{d P}{P}$ value varies from $-4 \%$ to $4 \%$ in steps of $2 \%$.

The $\frac{d P}{P}$ optimization results for the GMp experiment of April 2015 are shown in Fig. 5.23, which shows the discrepancy between the mean value of the Gaussian fitting of the reconstructed DpKin and DpKin ${ }_{\text {Real }}$. DpKin and DpKin Real $_{\text {are defined as }}$

$$
\begin{gathered}
\operatorname{DpKin}_{\text {Real }}=\frac{\mathrm{P}_{\theta_{\mathrm{HRS}}}-\mathrm{P}_{\text {Central }}}{\mathrm{P}_{\text {Central }}} \\
\text { DpKin }=\mathrm{dP}-\frac{\mathrm{P}_{\theta}-\mathrm{P}_{\text {Loss }}-\mathrm{P}_{\theta_{\mathrm{HRS}}}}{\mathrm{P}_{\text {Central }}}
\end{gathered}
$$

where $\mathrm{P}_{\text {Central }}$ is the HRS setting momentum and $\mathrm{P}_{\theta}$ is the scattering momentum of angle $\theta$ without energy loss.

Here the resolution from momentum calibration is $\sim 15 \times 10^{-4}$, much bigger than the 
spectrometer momentum resolution, which is $2 \times 10^{-4}$. It is because the optics calibration momentum resolution comes from multiple sources besides the spectrometer momentum resolution: beam energy uncertainty, scattering angle resolution, multiple scattering and radiative effects. Chap. 7.1 gives the comparisons of the invariant mass $\mathrm{W}$ between the Monte Carlo simulation and experimental data in the spring of 2015. The widths of W are in very good agreement and this indicates the resolutions applied in the Monte Carlo simulation, such as the beam energy, VDC tracking, are all reasonable. 


\section{CHAPTER 6}

\section{Data Analysis}

\subsection{Analysis Introduction}

In this chapter the event reconstruction procedure and the corrections and efficiencies applied to the number of the detected electrons, $N_{\text {electron }}$, will be described. These corrections and efficiencies include the VDCs tracking efficiency $\epsilon_{V D C}$, the computer and electronics live-times, $C L T$ and $E L T$ respectively, the trigger efficiency $\epsilon_{T}$, the particle identification (PID) efficiency $\epsilon_{P I D}$ and the target boiling correction $C_{T B}$. After applying all the above corrections and efficiencies, we can get the effective electron number $N_{\text {eff }}$ as defined below:

$$
N_{\text {eff }}=\frac{1}{p s}\left(N_{\text {electron }} \times E L T \times C L T \times \epsilon_{V D C} \times \epsilon_{T} \times \epsilon_{P I D} \times C_{T B}\right)
$$

where $p s$ is the prescale factor which was set to 1 for all the kinematics of experiment E12-07-108 during the spring of 2015, which are listed in Table 6.1. 
TABLE 6.1: Summary of the kinematics in the spring of 2015 for experiment E12-07-18.

\begin{tabular}{|c|c|c|c|c|c|c|c|}
\hline \multicolumn{9}{|c|}{ RIGHT ARM } \\
\hline kin & Run \# & $\begin{array}{c}E_{\text {beam }} \\
(\mathrm{GeV})\end{array}$ & $\Theta_{\text {HRS }}(\mathrm{deg})$ & $\begin{array}{c}E^{\prime} \\
(\mathrm{GeV})\end{array}$ & $\begin{array}{c}P_{H R S} \\
(\mathrm{GeV})\end{array}$ & $Q^{2}\left(\mathrm{GeV}^{2}\right)$ & $\epsilon$ \\
\hline$a$ & 21275 & 2.057 & $48.8^{\circ}$ & 1.177 & 1.152 & 1.652 & 0.578 \\
\hline
\end{tabular}

\begin{tabular}{|c|c|c|c|c|c|c|c|}
\hline \hline \multicolumn{8}{|c|}{ LEFT ARM } \\
\hline kin & Run \# & $\begin{array}{c}E_{\text {beam }} \\
(\mathrm{GeV})\end{array}$ & $\Theta_{\text {HRS }}(\mathrm{deg})$ & $\begin{array}{c}E^{\prime} \\
(\mathrm{GeV})\end{array}$ & $\begin{array}{c}P_{H R S} \\
(\mathrm{GeV})\end{array}$ & $Q^{2}\left(\mathrm{GeV}^{2}\right)$ & $\epsilon$ \\
\hline$b$ & 11386 & 2.057 & $45^{\circ}$ & 1.253 & 1.225 & 1.509 & 0.639 \\
$c$ & 11391 & 2.057 & $35^{\circ}$ & 1.473 & 1.443 & 1.096 & 0.789 \\
$d$ & 11413 & 2.057 & $25^{\circ}$ & 1.706 & 1.671 & 0.658 & 0.901 \\
\hline
\end{tabular}

\subsection{Event Reconstruction}

The data acquisition system (DAQ) in Hall A was used during the run of experiment E1207-108, which was controlled by the CEBAF Online Data Acquisition System (CODA) [4]. The raw data read from CODA was replayed into ROOT files using the standard Hall A physics analysis software, Analyzer [73]. The Analyzer decodes, filters and calibrates the hits from the detectors and reconstructs the physical variables of interest for the analysis on an event-by-event basis. These physical variables include VDC wire efficiency, reconstructed vertex positions, energy loss, etc.

\subsection{VDC tracking Efficiency}

The VDC tracking inefficiency is defined as the fraction of good events where we do not reconstruct good tracks in the VDCs. Each of the four planes of VDCs can fire one or several clusters of wires when an electron goes through. As a result, one or several tracks can be reconstructed, or no tracks are formed because of the inefficiency. In the analysis 
of the data from experiment E12-07-108 during the spring of 2015, only one-track events passing the PID cut (see Chap. 6.5) are defined as good events. Thus the VDC tracking efficiency $\epsilon_{V D C}$ is defined as the fraction of the $N_{\text {one }} / N_{\text {total }}$, where $N_{\text {one }}$ is the number of the one-track events passing the PID cuts and $N_{\text {total }}$ is the number of all the events which pass the PID cuts. The $\epsilon_{V D C}$ is calculated and applied to each run separately and the results are shown in Table 6.2.

TABLE 6.2: The VDC tracking efficiency for each run.

\begin{tabular}{|c|c|c|c|c|}
\hline Run number & 21275 & 11386 & 11391 & 11413 \\
\hline$\epsilon_{V D C}$ & 0.9975 & 0.9968 & 0.9949 & 0.9737 \\
\hline
\end{tabular}

Experiment E12-07-108 installed SCs for multi-track event analysis, but were not used in this thesis. Only one-track events were kept for the analysis in this thesis and the ratio of the multi-track events (see Table 6.2) is low, which is $<1 \%$ for three kinematics and $<3 \%$ for the other one). As a result, it will not improve the precision of the calculated cross section much even with the multi-track events and SC tracking information included. However, when E12-08-108 data at higher $Q^{2}$ is analyzed for high precision cross section calculation, the additional tracking information from the SCs will be very helpful. First, combing the tracking information from VDCs and SCs, the tracking efficiency of each VDC plane can be investigated. For example, the inefficiency of the VDC U1 plane can be calculated when a trajectory is formed by the clusters on the U2, V1, V2 planes and SCs while no cluster exists on the U1 plane. Second, the additional tracking information obtained from the SCs helps determine the right trajectory for a multi-track event. When several particles pass through the wire chambers simultaneously or if there are noisy wires, multi-track events can occur. For a multi-track event, two or more clusters can happen on each VDC plane and multiple trajectories can be formed by different combinations of the 
clusters. To determine which combination is the right one, a projection to the SCs plane can be made and if the corresponding straws are fired, it is certain that this combination of clusters gives a valid trajectory reconstruction.

\subsection{Trigger Efficiency}

The main trigger T1 of the experiment E12-07-108 was formed by the coincidence of the scintillators $S_{0}$ and $S_{2 m}$, or $S_{0} \& S_{2 m}$. To investigate the efficiency of T1, we can use trigger $\mathrm{T} 2$ and trigger T3, where T2 was formed by the coincidence of the scintillators $S_{0}$ and gas Cherenkov, $S_{0} \& G C$, and T3 was formed by the coincidence of the scintillators $S_{2 m}$ and gas Cherenkov, $S_{2 m} \& G C$. Then the $\epsilon_{T 1}$ can described as:

$$
\epsilon_{T 1}=\epsilon_{S_{0} \& S_{2 m}}=\frac{\left(S_{0} \& S_{2 m}\right) \&\left[\left(S_{0} \& S_{G C}\right) \|\left(S_{2 m} \& S_{G C}\right)\right]}{\left(S_{0} \& S_{G C}\right) \|\left(S_{2 m} \& S_{G C}\right)}
$$

Unfortunately during the spring of 2015, we only used triggers T1 and T3 and we can't get T1 efficiency from only T3. The efficiency study with the production data during 2016 spring and fall shows that the $\mathrm{T} 1$ efficiency is never lower than $99.5 \%$. So $\epsilon_{T 1}=99.5 \%$ is applied to all the kinematics for this thesis with a conservative uncertainty of $0.5 \%$.

\subsection{Particle Identification Efficiency (PID)}

The Cherenkov and electromagnetic calorimeter detectors are typically referred to as particle identification detectors (PID). The Cherenkov is a threshold detector designed that electrons will emit Cherenkov radiation while pions will not. The shower system provides

additional electron rejection capability beyond that of the Cherenkov by detecting the energy deposition of the particle. 


\subsubsection{Cherenkov}

\section{Detection Efficiency}

To determine the electron detecting efficiency of the Cherenkov detector, a sample of electron events that were observed in the lead-glass calorimeter is selected. The number of the events selected in this electron sample cut is called $\eta_{s h}$. Then the number of those events which trigger the Cherenkov is counted $\left(\eta_{c e r}\right)$. Thus the Cherenkov detection efficiency can be determined by Eq. 6.3 .

$$
\epsilon_{c e r}=\eta_{c e r} / \eta_{s h}
$$

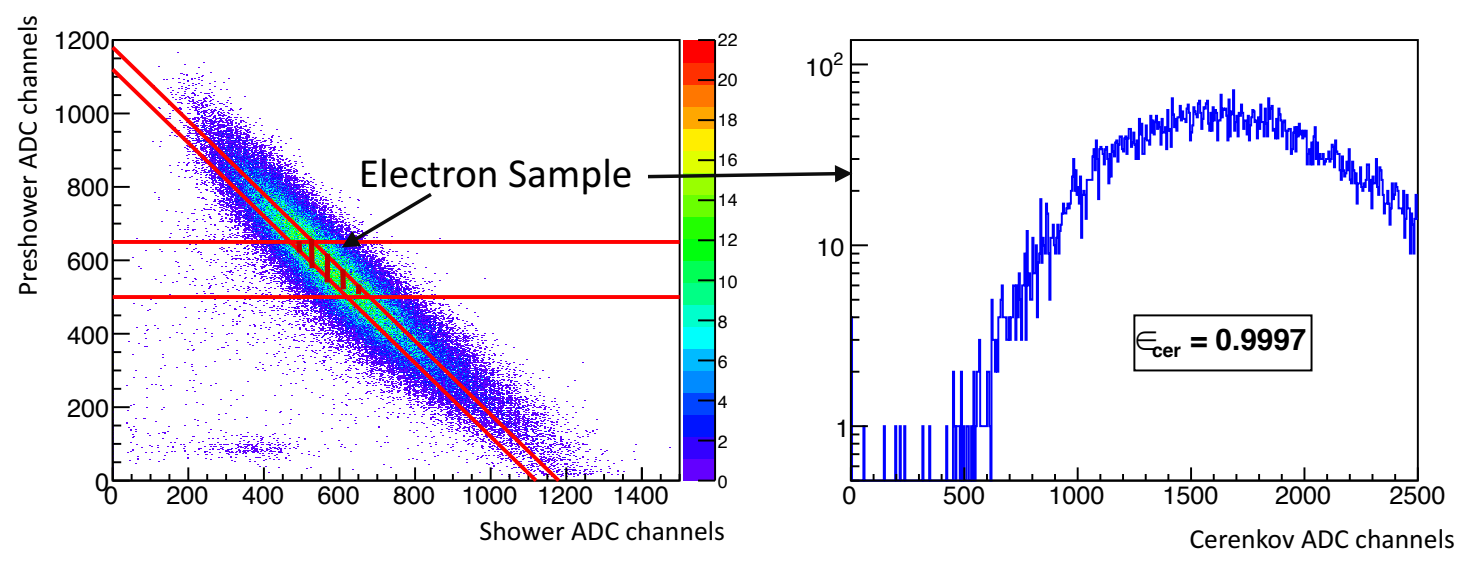

FIG. 6.1: Plots of the electron detecting efficiency of the Cherenkov detector. The left panel shows the electron sample cut made on the 2-D pre-shower vs shower plot. The right panel shows the Cherenkov spectrum of the electron sample. The detection efficiency of the Cherenkov is $99.97 \%$.

An example is given of the right arm spectrometer kinematics $a$. The left panel in Fig. 6.1 shows the electron sample cut and the right panel shows the corresponding electron sample on the Cherenkov. We found $\epsilon_{c e r}=99.97 \%$ for the right-arm. For the left arm 
Cherenkov, we found $\epsilon_{c e r}=100.00 \%$ for three kinematics.

\subsubsection{Cut Efficiency}

The goal of the PID analysis is to reject as many as pions as possible while keeping a high electron detection efficiency. To achieve this goal, a $\pi$ sample and an electron sample are selected in the 2D pre-shower/shower plot and then their distributions are observed in the Cherenkov spectrum. A Cherenkov cut is made to select the electrons and reject the pions. The electron detection efficiency and the pion rejection efficiency are calculated as below:

$$
\begin{gathered}
\epsilon_{e}=\frac{\text { Number of the electrons passing the Cherenkov cut in the electron sample }}{\text { Number of the events in the electron sample }} \\
\epsilon_{\pi}=\frac{\text { Number of the pions rejected by the Cherenkov cut in the pion sample }}{\text { Number of the events in the pion sample }}
\end{gathered}
$$

The Cherenkov cut was chosen to maintain a high electron detection efficiency (greater than 99.9\%) while obtaining a high pion rejection efficiency (greater than 99\%).

The R-arm Cherenkov cut efficiency of kinematic $a$ is shown in Fig. 6.2. The top two panels show the electron and $\pi$ samples selected from the $2 \mathrm{D}$ pre-shower/shower plot and the bottom panel shows the Cherenkov spectra of the two samples. The Cherenkov cut was placed at channel 300 and we found $\epsilon_{e^{-}}=99.93 \%$ and $\epsilon_{\pi}=100.00 \%$. Fig. 6.3 displays how the electron detection efficiency and pion rejection efficiency evolve as a function of the Cherenkov cut at different channels. The electron efficiency gets lower as the Cherenkov cut moves in the larger channel direction while the pion rejection efficiency gets higher; the Cherenkov cut was chosen to optimize both electron detection and pion rejection efficiency.

The Cherenkov cut efficiencies for each kinematic are listed in Table 6.3. These kinematics are elastic e-p scattering and thus most of the scattered particles are electrons. 
The $\pi$ rejection efficiency has a large uncertainty due to the low statistics of pions. Thus the $\pi$ rejection efficiency $97.04 \%$ for one of the kinematic doesn't mean it is not high enough.

R-arm Cherenkov cut efficiency
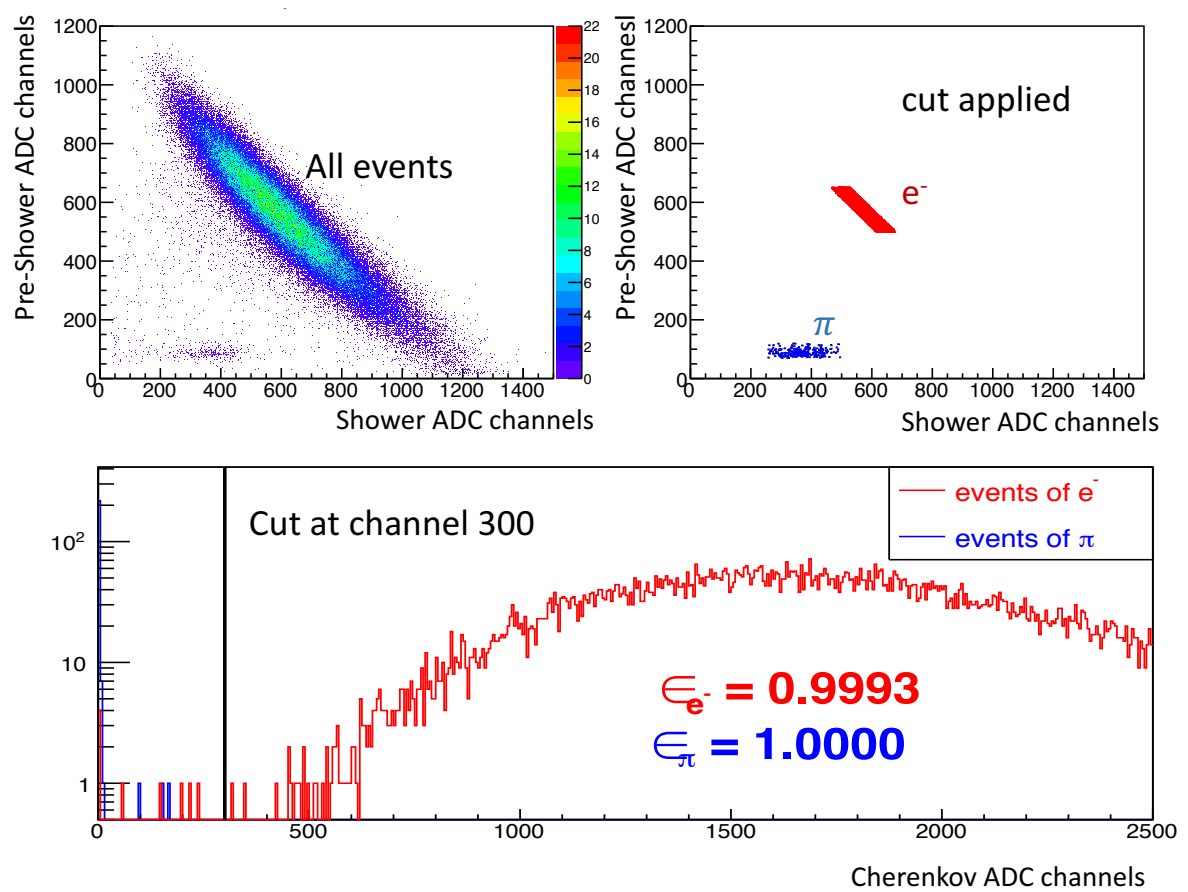

FIG. 6.2: Plots of the electron detecting and pion rejecting efficiencies of the Cherenkov detector. The top two panels show the electron and $\pi$ samples selected from the 2D pre-shower/shower plot and the bottom panel shows the Cherenkov spectra of the two samples.

\subsubsection{Electromagnetic Calorimeter}

\section{Detection Efficiency}

To determine the detector efficiency of the pre-shower/shower detectors, a selection of electrons that trigger the Cherenkov and are far above the single-photoelectron peak threshold is made. The number of the events selected in this cut is called $\eta_{c e r}$. Then the number of those events which trigger the pre-shower and shower is counted $\left(\eta_{s h}\right)$. Thus the 


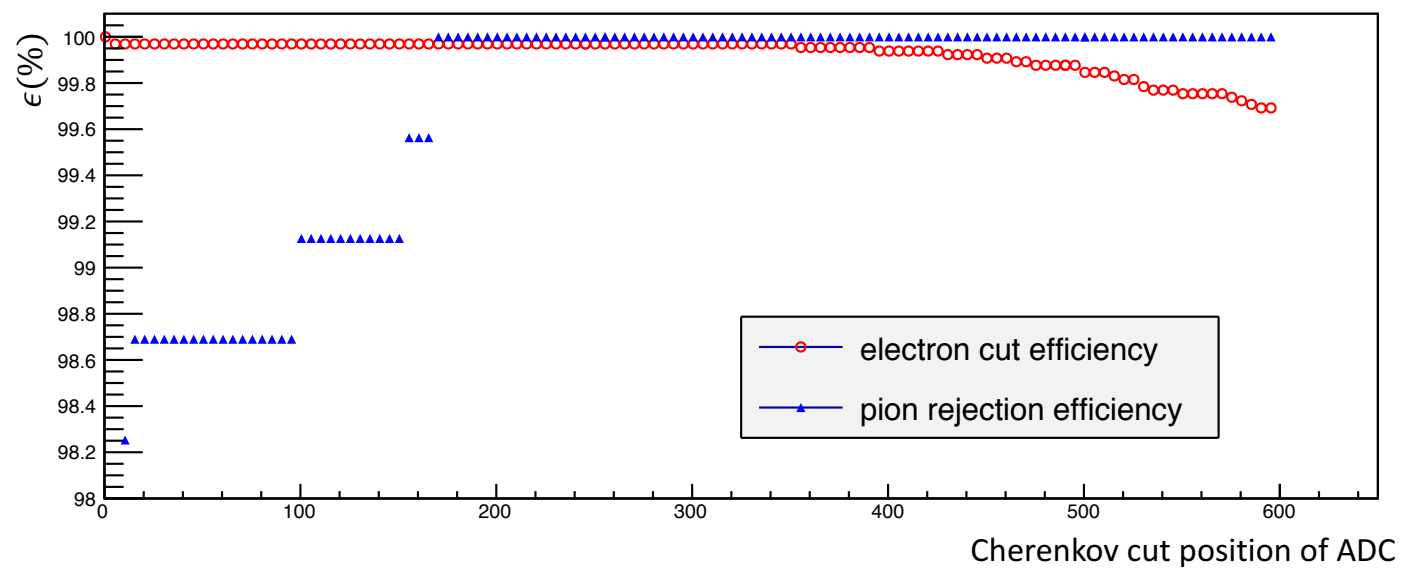

FIG. 6.3: The Cherenkov electron detecting and pion rejecting efficiencies vs. Cherenkov cut. The red circles show evolution of the electron detection efficiency with Cherenkov cut at different channels, and the blue triangles shows that of the pion rejection efficiency.

TABLE 6.3: Cut efficiency of Cherenkov on both spectrometer arms.

\begin{tabular}{|c|cc|ccc|cc|ccc|}
\hline \multicolumn{9}{|c|}{ RIGHT ARM } & \multicolumn{5}{c|}{ LEFT ARM } \\
\hline $\begin{array}{c}E \\
(\mathrm{GeV})\end{array}$ & $\begin{array}{c}\theta_{H R S} \\
\left.{ }^{o}\right)\end{array}$ & $\begin{array}{c}P_{0} \\
(\mathrm{GeV})\end{array}$ & cut & $\epsilon_{\text {cut }}\left(e^{-}\right)$ & $\epsilon_{r e j}(\pi)$ & $\theta_{H R S}$ & $\mathrm{P}_{0}$ & cut & $\epsilon_{\text {cut }}\left(e^{-}\right)$ & $\epsilon_{\text {rej }}(\pi)$ \\
$\%$ & $\%$ & $\left(^{o}\right)$ & $(\mathrm{GeV})$ & & $\%$ & $\%$ \\
\hline 2.057 & 48.8 & 1.152 & 300 & 99.93 & 100 & 45 & 1.225 & 300 & 99.96 & 99.64 \\
& & & & & & 35 & 1.443 & 300 & 99.95 & 97.87 \\
& & & & & & 25 & 1.671 & 300 & 99.97 & 100 \\
\hline
\end{tabular}


preshower/shower detection efficiency can be determined by Eq. 6.6.

$$
\epsilon_{s h}=\eta_{s h} / \eta_{c e r}
$$

An example is given of the right arm spectrometer for kinematics $a$. The left panel in Fig. 6.4 shows the selected electron sample (red shadow) and the right panel shows its distribution on the 2-D pre-shower/shower plot. We found $\epsilon_{s h}=99.89 \%$. The detection efficiencies of the left-arm total shower are all higher than $99.98 \%$ for each kinematic.
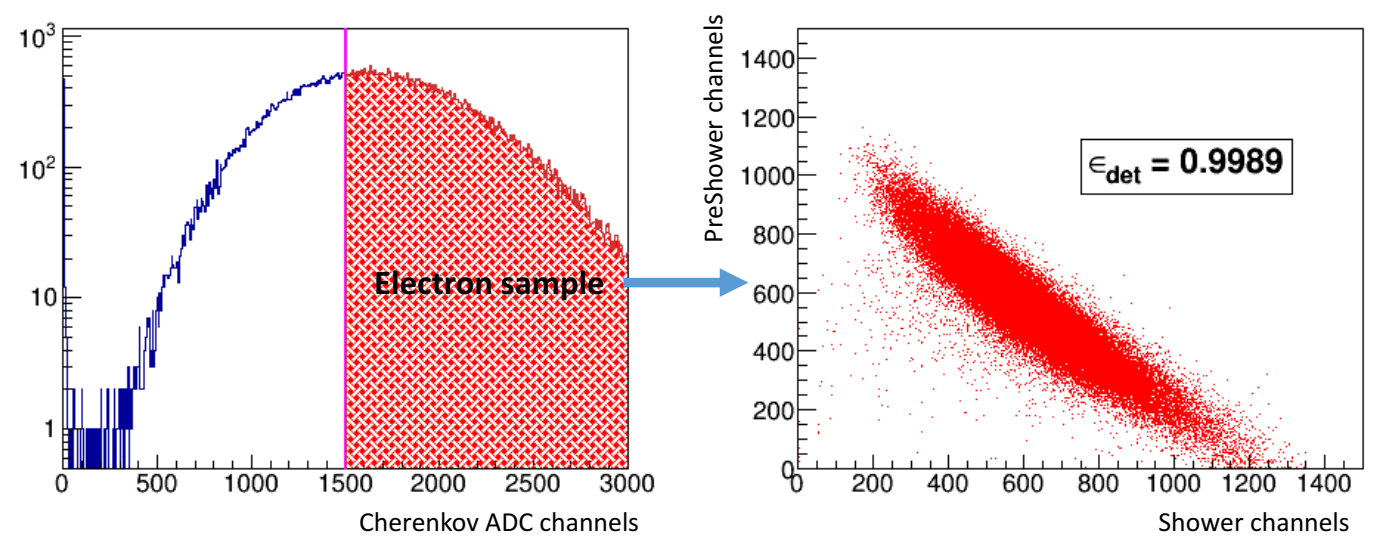

FIG. 6.4: Plots of the electron detecting efficiency of the shower. The left panel shows the electron sample cut made on the Cherenkov. The right panel shows the sample events on the pre-shower $v s$ shower plot. The shower detection efficiency is $99.89 \%$.

\subsubsection{Cut Efficiency}

To complement the Cherenkov cut, the PID analysis can be improved by applying two cuts on the EM calorimeters: a cut on the first layer of lead glass (pre-shower), and a cut on the total energy deposited in the calorimeter divided by the particle's momentum $(E / P)$. The two cuts are optimized to get a total efficiency greater than $99 \%$ as illustrated in 
Fig. 6.5. In the left panel of Fig. 6.5, Cherenkov and anti-Cherenkov cuts were applied to discriminate the electrons (in blue) and pions (in red). In the right panel, the calorimeter detection efficiency was calculated. The EM cut efficiency is calculated as below:

$$
N_{C e r}=\text { number of events passing the Cherenkov cut }
$$

$$
N_{P S H / S H}=\text { number of events that also pass the PSH and } E / P \text { cuts }
$$

$$
\epsilon_{e^{-}}=\frac{N_{P S H / S H}}{N_{C e r}}
$$
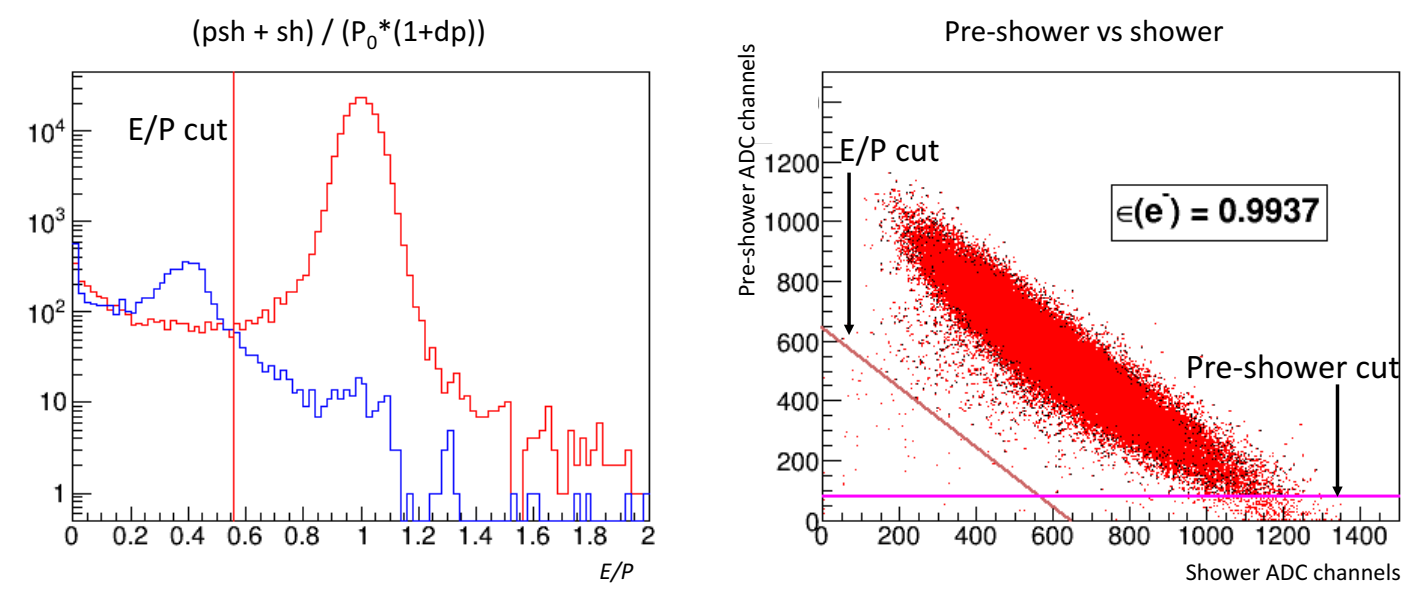

FIG. 6.5: Plots of the electron detecting efficiency of the shower and pre-shower. The left panel displays the $E / P$ spectra: the red line shows the events that pass the Cherenkov cut of 300 ADC channels and the blue line represents the events that fail it. The right panel shows them on the pre-shower/ shower $2 \mathrm{D}$ plot. The pre-shower cut and $E / P$ cut are both labeled on the right panel.

The two EM calorimeter cuts chosen for each kinematics and their efficiencies are listed in Table 6.4. 
TABLE 6.4: PID cuts summary

\begin{tabular}{|c|c|c|c|c|c|c|c|}
\hline \multicolumn{10}{|c|}{ RIGHT ARM } \\
\hline $\begin{array}{c}E \\
(\mathrm{GeV})\end{array}$ & $\begin{array}{c}P_{0} \\
(\mathrm{GeV})\end{array}$ & $\begin{array}{c}\text { PSH } / P \\
\text { cut }\end{array}$ & $\begin{array}{c}E / P \\
\text { cut }\end{array}$ & $\begin{array}{c}\text { Cer } \\
\text { cut }\end{array}$ & $\begin{array}{c}\epsilon_{e^{-}} \\
\text {EM cuts }\end{array}$ & $(\pi / \text { e })_{\text {before }}$ & $(\pi / e)_{\text {after }}$ \\
\hline 2.057 & 1.152 & 0.132 & 0.56 & 300 & 0.9937 & $(5.27 \pm 0.23) \times 10^{-3}$ & $(8.25 \pm 0.89) \times 10^{-3}$ \\
\hline \hline \multicolumn{8}{|c|}{ LEFT ARM } \\
\hline$E$ & $P_{0}$ & PSH $/ P$ & $E / P$ & Cer & $\epsilon_{e^{-}}$ & $(\pi / \text { e })_{\text {before }}$ & $(\pi / e)_{\text {after }}$ \\
$(\mathrm{GeV})$ & $(\mathrm{GeV})$ & cut & cut & cut & PID & & \\
\hline 2.057 & 1.225 & 0.122 & 0.40 & 300 & 0.9965 & $(3.50 \pm 0.12) \times 10^{-3}$ & $(7.17 \pm 0.55) \times 10^{-4}$ \\
& 1.443 & 0.104 & 0.40 & 300 & 0.9975 & $(3.51 \pm 0.12) \times 10^{-3}$ & $(7.18 \pm 0.55) \times 10^{-4}$ \\
& 1.671 & 0.090 & 0.42 & 300 & 0.9980 & $(3.63 \pm 0.12) \times 10^{-3}$ & $(8.16 \pm 0.59) \times 10^{-4}$ \\
\hline \hline
\end{tabular}

\subsubsection{Pion Suppresion}

The overall pion suppression can be examined by applying the three cuts described above and looking at the level of pion contamination that remains. The level of residual pion contamination is very low for all kinematics, as seen in Table 6.5.

TABLE 6.5: Pion contamination on both spectrometer arms with the three cuts mentioned above applied.

\begin{tabular}{|ccc|c|ccc|c|}
\hline \multicolumn{4}{|c|}{ RIGHT ARM } & \multicolumn{4}{c|}{ LEFT ARM } \\
\hline$E(\mathrm{GeV})$ & $\theta_{H R S}\left({ }^{\circ}\right)$ & $P_{0}(\mathrm{GeV})$ & $\epsilon_{\text {con }}(\pi)$ & $E(\mathrm{GeV})$ & $\theta_{H R S}\left(^{\circ}\right)$ & $\mathrm{P}_{0}(\mathrm{GeV})$ & $\epsilon_{\text {con }}(\pi)$ \\
\hline 1.057 & 48.8 & 1.152 & $4.9 \times 10^{-4}$ & 2.057 & 45 & 1.225 & $7.4 \times 10^{-4}$ \\
& & & & 2.057 & 35 & 1.443 & $5.6 \times 10^{-4}$ \\
& & & 2.057 & 25 & 1.671 & $4.9 \times 10^{-4}$ \\
\hline
\end{tabular}




\subsection{Computers and Electronics Deadtime}

The computer deadtime is the portion of time that the DAQ is busy writing an event when another event arrives. There is one scaler for each trigger type to count all the events fed to the trigger supervisor, which can be written as $N_{\text {Total }}^{i}$, where $i$ defines different trigger types. The prescalers of the E12-07-108 spring of 2015 elastic data were all set to 1 and

with the recorded events for each trigger type as $N_{D A Q}^{i}$, we can get the computer deadtime as $1-N_{D A Q}^{i} / N_{\text {Total }}^{i}$

The electronics deadtime is the portion of time that the hardware system is busy when another trigger comes in. When an event causes a trigger, a TDC gate is activated with the gate width $\tau$. If another event arrives and tries to activate the TDC gate within the time window $\tau$, it is ignored. If the event rate is $R$, then the probability of having $n$ counts in a time $t$ is given by the Poisson distribution $P(n)=(R t)^{n} e^{-R t} / n$ !. The electronics will be busy for $\tau$ for each event, thus the measured event rate $R_{m}$ is given by:

$$
\frac{R}{1+R \tau}=R_{m}
$$

and then livetime $t_{\text {live }}$ is given by:

$$
t_{\text {live }}=\frac{R_{m}}{R}=\frac{1}{1+R \tau}
$$

For experiment E12-07-108, the gates were about 60 ns. The electronics and computer dead-time were calculated for each run and the results are shown in Table 6.6. For run 21275 and 11386, the event rate is low and the electronics dead-time is negligible. 
TABLE 6.6: The computer and electronics deadtime for each kinematics.

\begin{tabular}{c|c|c|c|c}
\hline \hline Kinematics & $a$ & $b$ & $c$ & $d$ \\
\hline $\mathrm{DT}_{\text {computer }}(\%)$ & 34.61 & 5.96 & 25.68 & 86.03 \\
\hline $\mathrm{R}_{\text {scaler }}(\mathrm{Hz})$ & $250 \mathrm{~Hz}$ & $500 \mathrm{~Hz}$ & $2.8 \mathrm{kHz}$ & $30.8 \mathrm{kHz}$ \\
\hline $\mathrm{DT}_{\text {electronics }}(\%)$ & 0.00 & 0.00 & 0.02 & 0.18 \\
\hline
\end{tabular}

\subsection{Target Boiling Correction}

During experiment E12-07-108, a $\mathrm{LH}_{2}$ target was used. The density of the $\mathrm{LH}_{2}$ can decrease as the electron beam deposits heat into the target. This effect is referred to as target boiling. In order to study the boiling effect in the $\mathrm{LH}_{2}$, data were taken at various beam currents with both $\mathrm{LH}_{2}$ and carbon targets. The data taken with the carbon target should be current-independent since it is solid and melts only at $3800 \mathrm{~K}$.

To study how the target density $\rho$ changes due to the boiling effect, we define the normalized yield $Y$ as follows:

$$
Y=\frac{N_{\text {counts }}}{Q}
$$

where $\mathrm{N}_{\text {counts }}$ is the number of events from the scalers, corrected for current-independent rate (rate of cosmic events) and $Q$ is the beam charge. The normalized yield as a function of current should show a linearly decreasing relationship for $\mathrm{LH}_{2}$, so we can parameterize the target density $\rho$ as a function of current $I$ as:

$$
\rho_{I}=\rho_{0}\left(1+C_{T B} I\right)
$$

where $\rho_{0}$ is the target density at $I=0$ and $\mathrm{C}_{T B}$ is the target boiling correction. When we plot the defined yield as a function of current and make a linear fit, the slope gives $\mathrm{C}_{T B}$ 
as shown in Fig. 6.6. For carbon, the slope is consistent with zero $(-0.8 \pm 0.5) \% / 100 \mu \mathrm{A}$ as it should be. For $\mathrm{LH}_{2}$, the slope is $(-2.7 \pm 0.4) \% / 100 \mu \mathrm{A}$. Table 6.7 lists the average beam current $I_{\text {beam }}$ for each kinematics in this thesis and the corresponding target boiling correction $C_{T B}$.

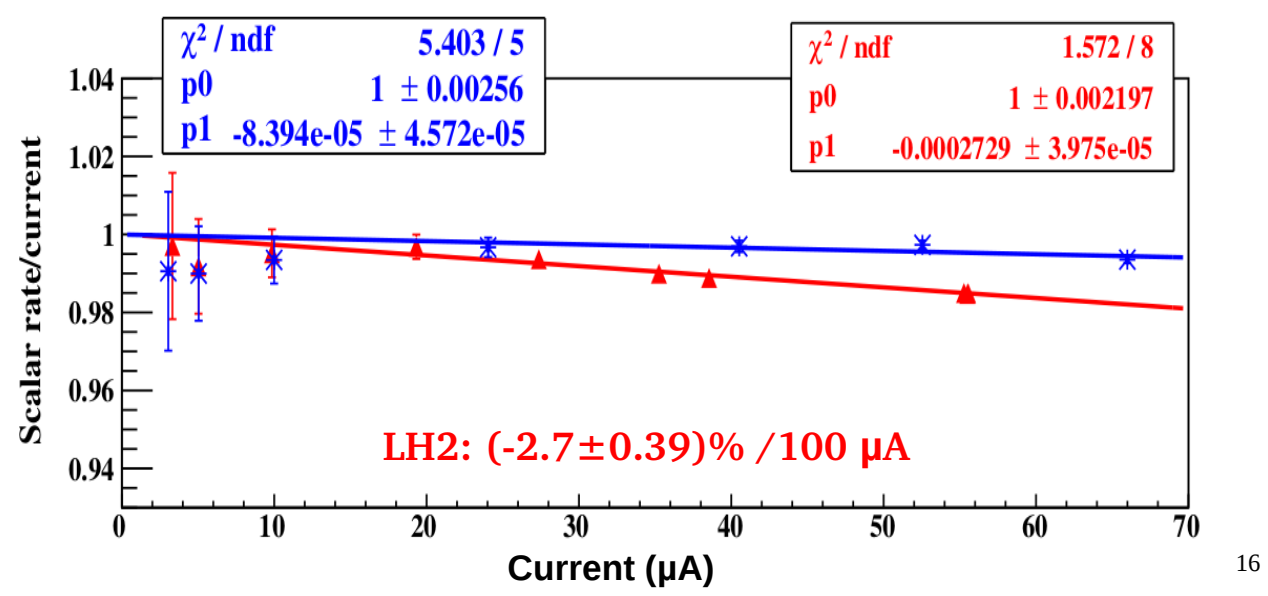

FIG. 6.6: Target boiling study. The normalized yield for $\mathrm{LH}_{2}$ target (red line) and carbon (blue line) as a function of beam current. The plot is made by Thir Gautam.

TABLE 6.7: The average beam current $I_{\text {beam }}$ and target boiling correction $C_{T B}$ for each kinematics.

\begin{tabular}{c|c|c}
\hline \hline Kinematics & $I_{\text {beam }}(\mu A)$ & $C_{T B}$ \\
\hline$a$ & 16.53 & $(-0.45 \pm 0.06) \%$ \\
\hline$b$ & 16.27 & $(-0.44 \pm 0.06) \%$ \\
\hline$c$ & 18.22 & $(-0.49 \pm 0.07) \%$ \\
\hline$d$ & 20.00 & $(-0.54 \pm 0.08) \%$ \\
\hline
\end{tabular}

\subsection{Monte-Carlo Simulations}

For a fixed angle and momentum setting, the HRS can only detect events within a limited range around the central momentum $( \pm 4.5 \%)$ and the central angle $(\sim 6 \mathrm{msr})$. However, 
not all the electrons within this certain range can be transported through the spectrometer and some of them are lost due to scraping the walls of the magnets and hitting the vacuum tubes and apertures between them. In the simplest picture, the probability that an electron event can be accepted by the spectrometer within a certain phase space (defined by $\delta, x_{\text {tar }}^{\prime}$ and $\left.y_{t a r}^{\prime}\right)$ can be defined as the spectrometer acceptance. To obtain the elastic $e-p$ scattering cross section, the Monte Carlo simulation program SIMC was used to model the acceptance for both HRS arms for the experiment E12-07-108 data analysis.

SIMC is the standard Hall A and Hall C Monte Carlo simulation program for coincidence reactions (experiment E12-07-108 only detected electrons in one arm of the spectrometers and thus only part of the simulation, the electron detecting arm, was used for analysis). SIMC was adapted from the simulation program SIMULATE which was written for SLAC experiment NE18 [74, 75]. SIMC has three main components: the event generator, the spectrometer model, and a list of materials and apertures. The event generator includes the cross-section model weighting and radiative corrections and the spectrometer model simulates the transportation of the particles through the magnets. The list of materials and apertures are those that can cause multiple scattering or stop the particles through the transportation.

First, SIMC generates the positions of the electrons at the target randomly based on the beam positions, the beam width, the raster size and the target geometry. The generated beam energy spread is matched with the actual beam and then the beam energy is corrected for the ionization losses in the target before the interaction. Then SIMC generates the scattering angles with a flat distribution in $x_{t a r}^{\prime}$ and $y_{t a r}^{\prime}$, which populates $100 \%$ of the actual spectrometer acceptance. In our elastic scattering case, the energy of the scattered electron is calculated. For each event, it is weighted with the cross section model [76] and then radiative corrections are included. After this, the scattered electron is transported through the target, the magnets and a list of materials, and the ionization energy loss and 
multiple scattering effects are applied. Finally, only the scattered electrons that reach the detectors are recorded.

The optical properties of the magnets of HRS are modeled with the Monte Carlo simulation COSY INFINITY program [77]. COSY generates two matrices, the forward and backward matrices, using a list of the magnets' parameters such as their positions, dimensions and field maps. The forward matrix transports the interaction variables at the target to the focal plane and the backward reconstruct them at the target through the tracks at the focal plane. COSY also generates forward matrices which transports the interaction variables from the target to some other positions besides the focal plane. These positions are $2 / 3$ along the way through each quadrupole, and, the end of each quadrupole and dipole ( $Q Q D Q$ configuration). SIMC checks whether the electrons pass the beginning and end of the magnets to make sure they are within the acceptance of the magnets and the trajectories at $2 / 3$ of the magnets are calculated to get rid of the events that scrape the magnets' walls. The vacuum tubes and all the apertures in the spectrometers are added in SIMC based on their actual positions and sizes. SIMC assures the electrons go through them successfully by projecting the rays to where they are located and throw away the events which are outside of the acceptance. The detector stack is included in SIMC and fiducial cuts are applied according the sizes and positions of the detectors. In addition, VDCs smearing is applied to the electron positions at the two VDCs to match those of the actual VDCs. The backward matrix elements then reconstruct the focal plane variables to the interaction variables at the target.

To investigate the uncertainties from the $\mathrm{MC}$, the ratio of the $\mathrm{MC}$ and data normalized yields was obtained (see Chap. 7.1). Then each aperture in the MC was moved by $1 \mathrm{~mm}$ in the vertical or horizontal direction and how much the ratio of the data and MC yields changed was calculated; each aperture was moved by $1 \mathrm{~mm}$ because we think the accuracy of the aperture's position is $\sim 1 \mathrm{~mm}$. Four movements were made for each aperture, up 
and down in the vertical direction and left and right in the horizontal direction, and the uncertainty caused by the displacement in each direction was determined by the bigger change. Then the strength of the magnetic fields of Q1 and Q3 were changed by increasing or decreasing $1 \%$ and that of Q2 was changed by $0.3 \%$, where $1 \%$ and $0.3 \%$ were considered as the field strength uncertainties of Q1, Q3, and Q2 respectively. The uncertainty of each quadrupole caused by the change of the field strength was determined by the bigger one of the increase and decrease. The uncertainties caused by each aperture's displacement and the magnetic field strength change of each quadrupole are listed in Appendix B. Combining all the uncertainties obtained from moving each aperture and changing each magnet field's strength, a total uncertainty from the $\mathrm{MC}$ of $2.15 \%$ is obtained. For the discrepancy in the LHRS between the MC and the experimental data (see Fig. 5.20), a conservative uncertainty of $4 \%$ was applied, which is considered as a reasonable number to account for the discrepancy.

\subsection{Radiative Corrections}

In Chap. 1, the cross section corresponding to one-photon-exchange, or the Born approximation, is derived to the lowest-order of the electromagnetic coupling constant $\alpha$ and expressed using the Sachs form factors as in Eq. 1.22. The Feynman diagram of the one-virtual-photon-exchange is shown in Fig. 1.2. However, higher order processes could happen in which that the electron or the proton radiates additional real or virtual photons. The measured cross section needs to be corrected for the effects from these radiations and these corrections are referred to as radiative corrections. They are classified into two categories: the external and the internal radiative corrections.

The external radiative corrections come from the process that the electron radiates bremsstrahlung photons when traveling though materials before and after the scattering. 

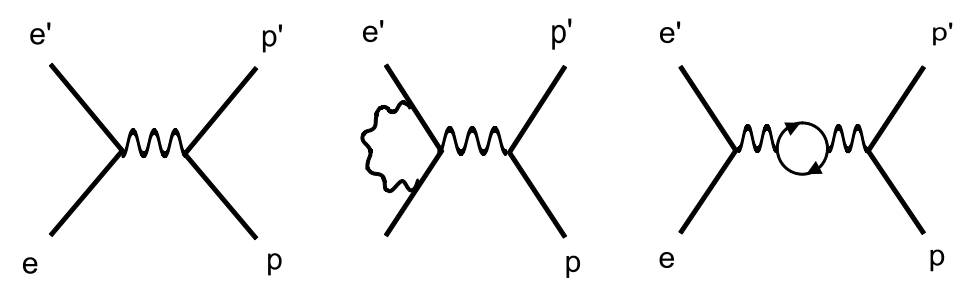

a) Born term

b) vertex

c) vacuum
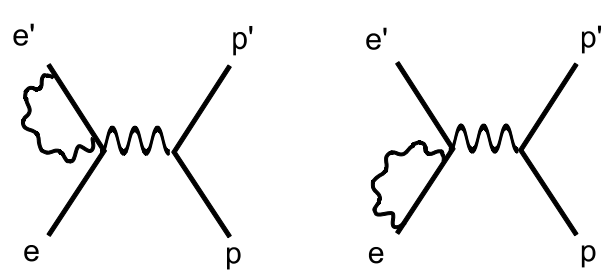

d) self energy
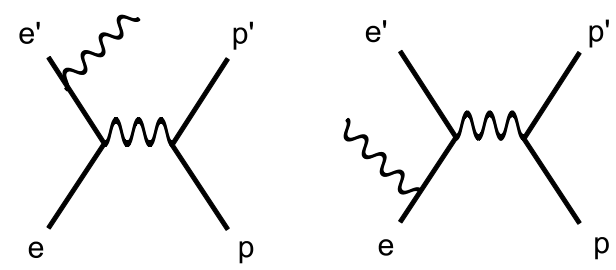

e) Bremsstrahlung

FIG. 6.7: Born term and lowest order radiative correction graphs for the electron in elastic $e p[5]$. 
The bremsstrahlung radiation is due to the deceleration of the electron as it approaches the Coulomb field of another particle. If the radiation comes from the interaction between the electron and a nucleus other than the one in the primary scattering vertex, it is the external radiation.

The internal radiative corrections are from processes when the electron interacts with the Coulomb field of the primary nucleus, the one involved in the scattering, and radiates real photons and emits and reabsorbs virtual photons. The various internal radiative correction diagrams involving the electron are shown in Fig. 6.7 [5]: (b) vertex, (c) vacuum polarization, (d) self energy, (e) internal bremsstrahlung. High-order process involve emission of multiple real photons and exchange of more than two virtual photons are all possible. Similar diagrams for the proton are shown in Fig. 6.8 [5]: (a) internal bremsstrahlung, (b) vertex, (c) self energy, (d) two photon exchange (TPE).

These internal radiative corrections can be classified into two categories: the elastic ones which only involve the virtual photons and the inelastic ones that emit real photons. The elastic internal radiative corrections depend exclusively upon $Q^{2}$ and are $\epsilon$ independent, thus they only affect the magnitude of the cross section. Taking a look at the cross section expression with Sachs form factors in Eq. 1.22, $G_{E p}^{2}$ and $G_{M p}^{2}$ are proportional to the slope and intercept of the linear fit of the cross section to $\epsilon$ at fixed $Q^{2}$ value, respectively. As a result, $G_{M p}^{2}$ is $G_{E p}^{2}$ stays the same but $G_{M p}^{2}$ is different when only the magnitude of the cross section changes for the elastic processes. The inelastic internal radiative and external radiative corrections involve real photon emissions. They make the incoming electron energy at the scattering vertex smaller than the beam energy and the detected electron energy smaller than the scattered electron energy at the vertex. Thus they are $\epsilon$ dependent and also modify the value of $Q^{2}$.

To obtain the single-photon-exchange cross section, also called the Born cross section, from the measured one, the radiative correction procedure from Mo and Tsai [78] and 

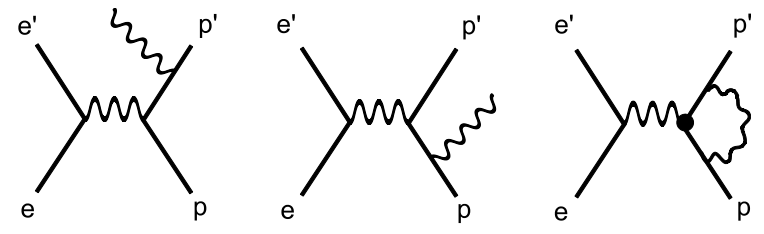

a) bremsstrahlung

b) vertex

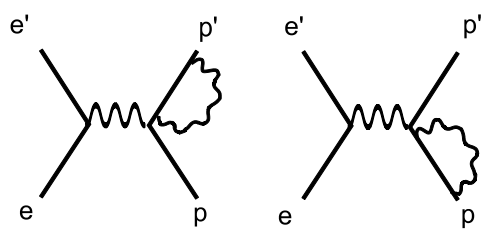

c) self energy
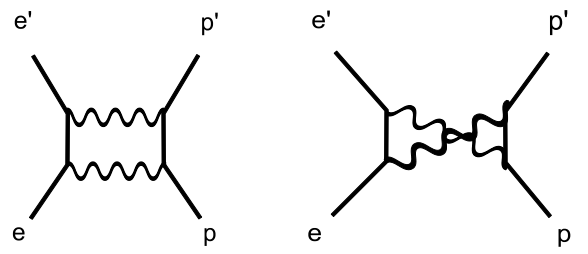

d) two-photon

FIG. 6.8: Lowest order radiative correction graphs for the proton in elastic ep [5]. 
modified by Walker $[79,1]$ and Ent $[74,80]$ was implemented in SIMC. The relation between them is as follows:

$$
\begin{aligned}
\left(\frac{d \sigma}{d \Omega}\right)_{\text {Measured }} & =\left(1+\delta_{\text {corr }}\right)\left(\frac{d \sigma}{d \Omega}\right)_{\text {Born }} \\
& =\left(1+\delta_{i n t}+\delta_{i n t^{\prime}}+\delta_{\text {ext }}\right)\left(\frac{d \sigma}{d \Omega}\right)_{\text {Born }}
\end{aligned}
$$

where $\delta_{\text {int }}$ and $\delta_{\text {ext }}$ represent the internal and external radiative corrections respectively and $\delta_{i n t^{\prime}}$ is from the improvement to the internal radiative corrections made by Walker. For corrections higher than $O\left(\alpha^{3}\right)$, the relation between them is written as:

$$
\left(\frac{d \sigma}{d \Omega}\right)_{\text {Measured }}=e^{\delta_{\text {corr }}}\left(\frac{d \sigma}{d \Omega}\right)_{\text {Born }}
$$

The internal radiative corrections applied in SIMC include: the electron and proton vertex corrections, the internal bremsstrahlung, the vacuum polarization and TPE. The external corrections are also made based on the target material and geometry and the spectrometer material. The systematic uncertainties from the radiative corrections are estimated to be less than $1 \%$ [81]. When the cut of the invariant mass $\mathrm{W}<1.07 \mathrm{GeV}$ is applied, the radiative correction $\delta_{\text {corr }}$ in SIMC for each kinematics is listed in Table 6.8. The value of $1.07 \mathrm{GeV}$ is called the pion threshold because $M_{p}+M_{\pi} \sim 1.07 \mathrm{GeV}$ and the pions can be created when $\mathrm{W}$ passes the pion threshold.

TABLE 6.8: The radiative correction $\delta_{\text {corr }}$ in SIMC for each kinematics, where the cut of the invariant mass $\mathrm{W}<1.07 \mathrm{GeV}$ is applied.

\begin{tabular}{c|c|c}
\hline \hline Kinematics & $\left(\frac{d \sigma}{d \Omega}\right)_{\text {Born }} /\left(\frac{d \sigma}{d \Omega}\right)_{\text {Measured }}$ & $\delta_{\text {corr }}$ \\
\hline$a$ & 1.178 & $-0.151 \pm 0.010$ \\
$b$ & 1.156 & $-0.135 \pm 0.010$ \\
$c$ & 1.174 & $-0.148 \pm 0.010$ \\
$d$ & 1.164 & $-0.141 \pm 0.010$ \\
\hline
\end{tabular}




\subsection{Cryogenic Target Aluminum Cell}

The cryogenic target used in experiment E12-07-108 is liquid hydrogen contained inside a cylindrical aluminum cell with a hemispherical tip. The entrance window and the exit window (the semispherical tip) are $0.175 \mathrm{~mm}$ and $0.132 \mathrm{~mm}$ thick respectively, and their densities are $2.81 \mathrm{~g} / \mathrm{cm}^{3}$. Their contributions are subtracted by using a $15 \mathrm{~cm}$ dummy target, which consists of two aluminum foils located at $\pm 7.5 \mathrm{~cm}$ along the hall coordinate system $z$ direction, corresponding to that of the cryotarget entrance and exit windows. The dummy target has the same density as the windows and its total thickness is 1.71 $\mathrm{mm}$.

To subtract the window contributions, the dummy and cryogenic target data are analyzed with the identical cuts applied and then normalized. The dummy data also needs to be scaled with the thickness ratio $T_{\text {window }} / T_{\text {dummy }}$, where $T_{\text {window }}$ and $T_{\text {dummy }}$ are the window and dummy foil thickness respectively. An example (kinematics $a$ ) is given in Fig. 6.9, where the top panel shows the normalized yields for the upstream dummy target, the downstream target, both of them together, and the liquid hydrogen target. The bottom panel shows the liquid hydrogen $\mathrm{LH}_{2}$ data with the dummy contribution subtracted; the contribution totalled $1.25 \%$ for kinematics $a$. The contributions for each kinematics are listed in Table 6.9.

TABLE 6.9: The dummy contribution for each kinematics.

\begin{tabular}{c|c}
\hline \hline Kinematics & $\frac{\text { dummy }_{2}(\%)}{\mathrm{LH}_{2}}$ \\
\hline$a$ & 1.25 \\
$b$ & 1.51 \\
$c$ & 2.10 \\
$d$ & 1.62 \\
\hline
\end{tabular}



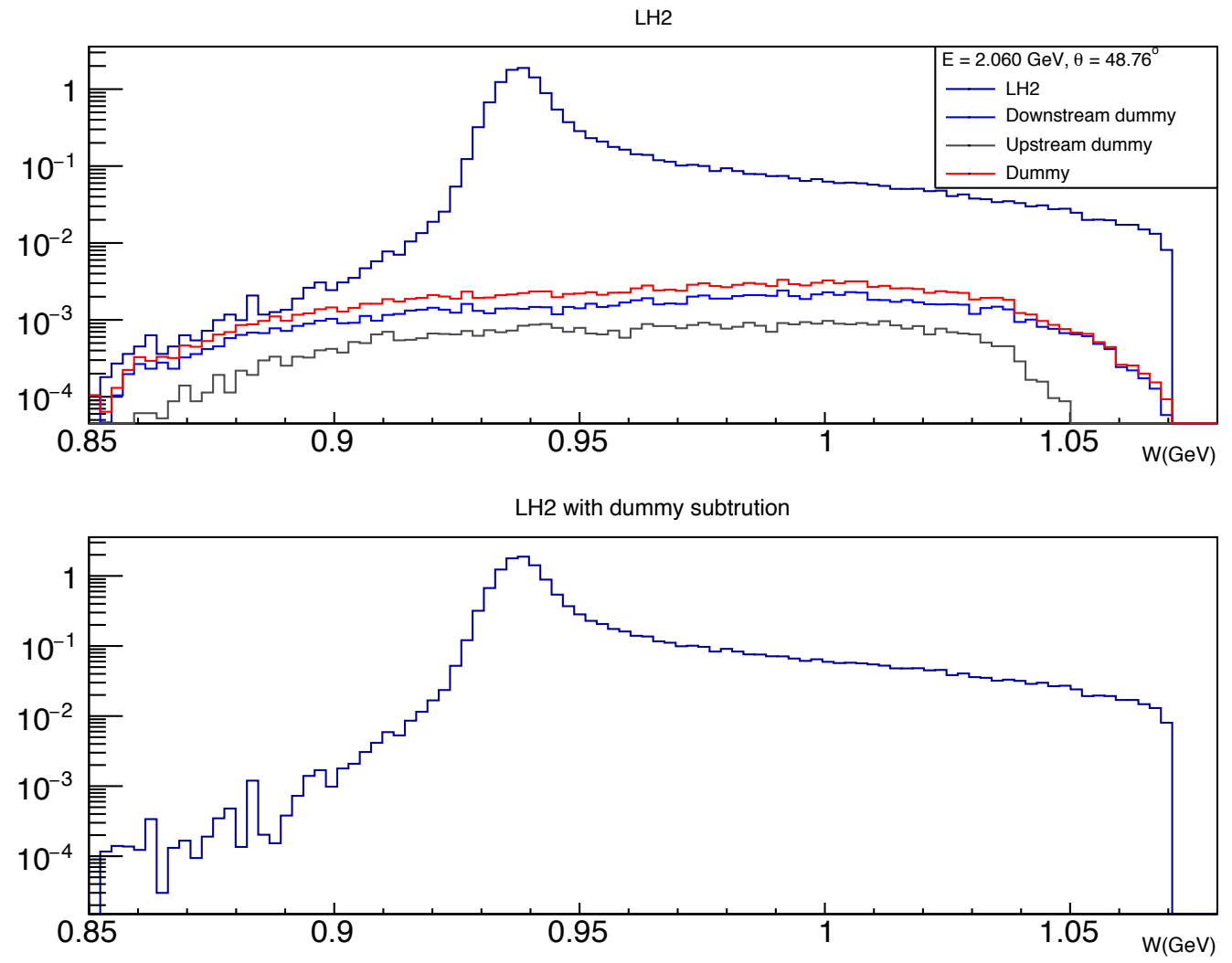

FIG. 6.9: The liquid hydrogen $\mathrm{LH}_{2}$ and dummy target data yields for kinematics $a$. The top panel shows the normalized yields for the upstream dummy target, the downstream target, two of them together, and the liquid hydrogen target. The bottom panel shows the liquid hydrogen $\mathrm{LH}_{2}$ data with the dummy contribution subtracted. 


\subsection{Summary of the Uncertainties}

Table 6.10 gives the summary of the uncertainties in the extracted cross section for each kinematics. Note that a conservative $4 \%$ uncertainty is applied to the three L-arm spectrometer kinematics, $b, c$ and $d$, due to the Monte Carlo simulation. This is from the discrepancy between the simulation and experimental data (see Chap. 7.1).

TABLE 6.10: The summary of the uncertainties in the extracted cross section for each kinematics.

\begin{tabular}{|c|c|c|c|c|}
\hline \hline Kinematics & $a$ & $b$ & $c$ & $d$ \\
\hline Spectrometer angle & $0.11 \%$ & $0.22 \%$ & $0.29 \%$ & $0.39 \%$ \\
Beam energy & $0.29 \%$ & $0.28 \%$ & $0.27 \%$ & $0.23 \%$ \\
BCM calibration & $1 \%$ & $1 \%$ & $1 \%$ & $1 \%$ \\
Target boiling & $0.06 \%$ & $0.06 \%$ & $0.07 \%$ & $0.08 \%$ \\
Trigger efficiency & $0.5 \%$ & $0.5 \%$ & $0.5 \%$ & $0.5 \%$ \\
Radiative corrections & $1 \%$ & $1 \%$ & $1 \%$ & $1 \%$ \\
Monte Carlo simulation & $2.15 \%$ & $4 \%$ & $4 \%$ & $4 \%$ \\
Statistical & $0.26 \%$ & $0.17 \%$ & $0.09 \%$ & $0.10 \%$ \\
\hline Total & $2.64 \%$ & $4.26 \%$ & $4.26 \%$ & $4.27 \%$ \\
\hline
\end{tabular}




\section{CHAPTER 7}

\section{Results}

\subsection{Reduced Cross Section $\sigma_{R}$ Extraction}

The measured elastic cross section can be calculated by:

$$
\left.\frac{d \sigma}{d \Omega}\right|_{\text {Measured }}=\frac{N_{\text {electron }}}{\Delta \Omega \operatorname{LT} \int L d t \epsilon_{T} \epsilon_{V D C} \epsilon_{P I D} C_{T B}}
$$

where $N_{\text {electron }}$ is the number of the electrons detected within the chosen acceptance and detector cuts and has subtracted the background from the target cell; $\Delta \Omega$ is the solid angle acceptance; $\epsilon_{T}, \epsilon_{V D C}$ and $\epsilon_{P I D}$ are the trigger efficiency, VDC tracking efficiency and particle identification efficiency, respectively; $C_{T B}$ is the target boiling correction; LT is the livetime; $\int L d t$ is the integrated luminosity, which can be calculated as:

$$
\int L d t=\frac{Q}{e} \rho_{T} \Delta L \frac{Z}{A} N_{A}
$$

where $Q=\int I_{\text {beam }} d t$ is the integrated beam charge with $I_{\text {beam }}$ being the electron beam current; $\rho_{T}$ is the target density; $N_{A}$ is Avogadro's number; $Z$ is the atomic number and 
$A$ is the atomic mass of the target; $\Delta L$ is the target length seen by the spectrometer. To convert the measured cross section to the single-photon-exchange cross section, the Born cross section of $O(\alpha)$, radiative corrections needed to be applied (see Chap.. 6.9):

$$
\left.\frac{d \sigma}{d \Omega}\right|_{\text {Measured }}=\left.\left(1+\delta_{\text {corr }}\right) \frac{d \sigma}{d \Omega}\right|_{\text {Born }}
$$

where $\delta_{\text {corr }}$ represent the radiative corrections.

During the analysis, the efficiency-corrected electron yield is used which is defined as:

$$
Y=\frac{N_{\text {electron }}-N_{B}}{\mathrm{LT} \epsilon_{\text {det }}}=\left(\int L d t\right) \frac{d \sigma}{d \Omega} \Delta \Omega A(\theta)
$$

where $A(\theta)$ is the spectrometer acceptance (see Chap. 6.8) which is dependent on the kinematics and thus dependent on the spectrometer central angle $\theta$. Similarly, the electron yield of the Monte Carlo simulation is defined as:

$$
Y_{M C}=N_{\text {electron }}(M C)=\left(\int L_{M C} d t\right) \frac{d \sigma}{d \Omega} \Delta \Omega A_{M C}(\theta)
$$

In Chap. 6.8, it has been discussed in detail how the spectrometer acceptance is modeled with the Monte Carlo simulation; if the MC does a good job, the assumption $A(\theta)=A_{M C}(\theta)$ can be made. Taking the ratio of the normalized yields, the relation between the measured and MC cross section can be written as:

$$
\left(\frac{d \sigma}{d \Omega}\right)_{\text {Measured }}=\left(\frac{d \sigma}{d \Omega}\right)_{\text {mod }}\left(1+\delta_{\text {corr }}^{M C}\right) \frac{Y(\theta)}{Y_{M C}(\theta)}
$$

where $\left(\frac{d \sigma}{d \Omega}\right)_{m o d}$ is the cross section given by the model used in the MC and $\left(1+\delta_{\text {corr }}^{M C}\right)$ accounts for the radiative corrections applied in the MC. However, the radiative corrections in the MC doesn't include the hard two-photon exchange, thus a parametrization of the 
elastic cross sections without TPE corrections applied to the data from [28] is used as follows:

$$
\sigma_{R}^{B o r n+T P E}=F_{M p}^{2}\left(Q^{2}\right)+\frac{\epsilon}{\tau} F_{E p}^{2}\left(Q^{2}\right)
$$

where $F_{M p}$ and $F_{E p}$ are the effective magnetic and electric form factors respectively and here their dependences on $\epsilon$ are neglected. The two effective form factors absorb the effects of multiple photon exchange and they are parametrized as follows [28]:

$$
\begin{gathered}
F_{E p}\left(Q^{2}\right)=\frac{1-1.651 \tau+1.287 \tau^{2}-0.185 \tau^{3}}{1+9.531 \tau+0.591 \tau^{2}+4.994 \tau^{5}} \\
\frac{F_{M p}\left(Q^{2}\right)}{\mu_{p}}=\frac{1-2.151 \tau+4.261 \tau^{2}+0.159 \tau^{3}}{1+8.647 \tau+0.001 \tau^{2}+5.245 \tau^{3}+82.817 \tau^{4}+14.191 \tau^{5}}
\end{gathered}
$$

where $\tau=Q^{2} / 4 M_{p}^{2}$. Note that this is a purely phenomenological fit form rather than a true representation of the effect of two-photon exchange.

As a result, the reduced cross section $\sigma_{R}$ can be taken as the value of the reduced cross section from the above parametrized form factors scaled by the ratio $R$ :

$$
\sigma_{R}=R \sigma_{R}(\mathrm{MC})=R\left(F_{M p}^{2}\left(Q^{2}\right)+\frac{\epsilon}{\tau} F_{E p}^{2}\left(Q^{2}\right)\right)
$$

where $R=Y / Y_{M C}$.

Fig. 7.1 to Fig. 7.4 show the comparison between the Monte Carlo simulation and the experimental data of the invariant mass $W$ and four variables in the target coordinate system (see Chap. 4.3): $\delta p / p$, in-plane-of angle $\phi$, out-plane-of angle $\theta$, and $y_{t g}$. A pion threshold cut $W<1.07 \mathrm{GeV}$ is applied. To obtain the reduced cross section $\sigma_{R}$ with the method above, all of them should match well to apply the assumption $A(\theta)=A_{M C}(\theta)$. Fig. 7.1 shows that the Monte Carlo simulation and experimental data are in a good agreement except a small discrepancy near the upper edge of the out-plane-of angle $\theta$, 

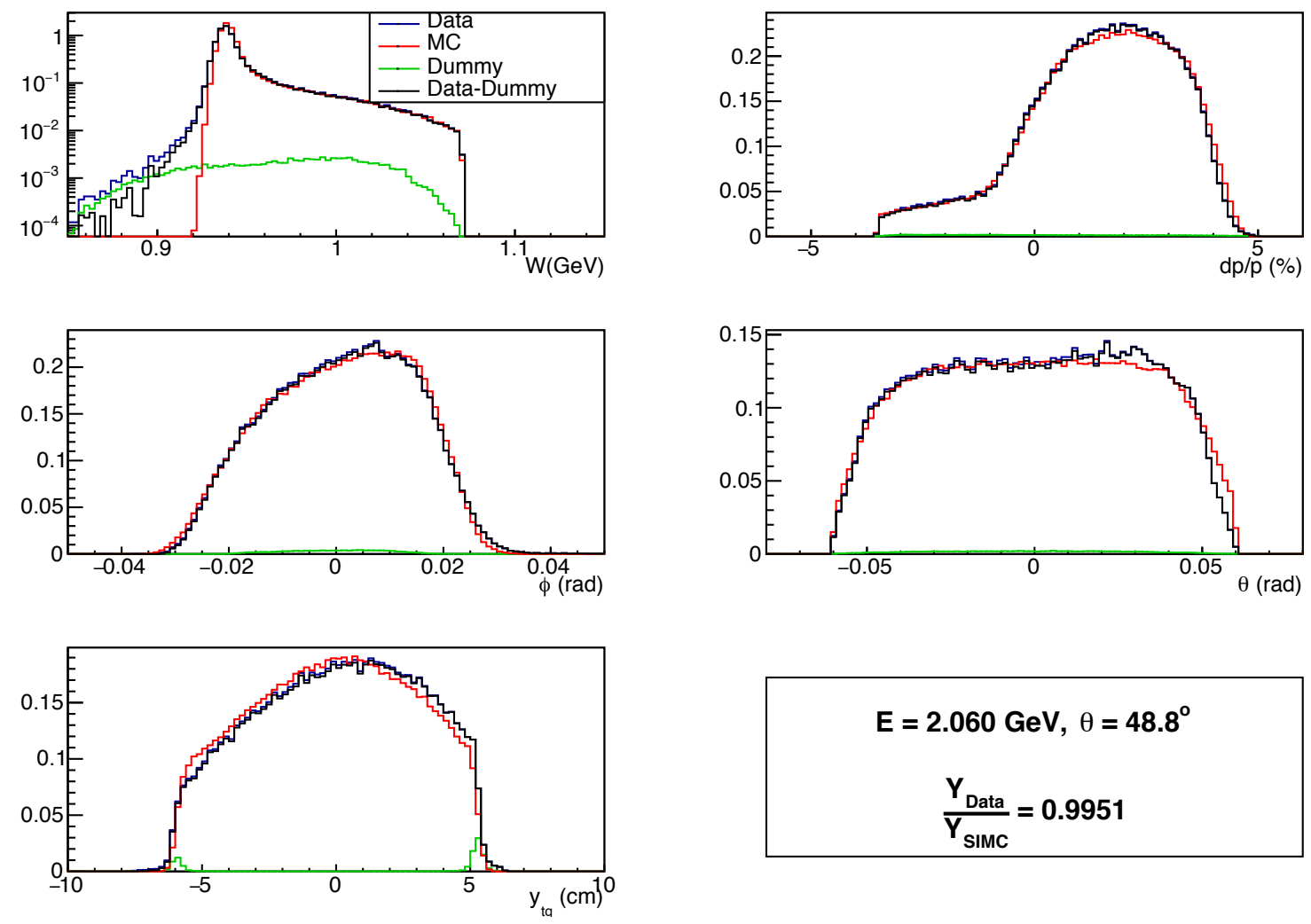

FIG. 7.1: Data yield compared to Monte Carlo yield of kinematics a (see Table 6.1). The variables are in the target coordinate system and a pion threshold cut $W<1.07 \mathrm{GeV}$ is applied. The blue line shows the data before the dummy subtraction; the green line shows the dummy yield; the black line shows the data after the dummy subtraction; the red line shows the Monte Carlo yield. 

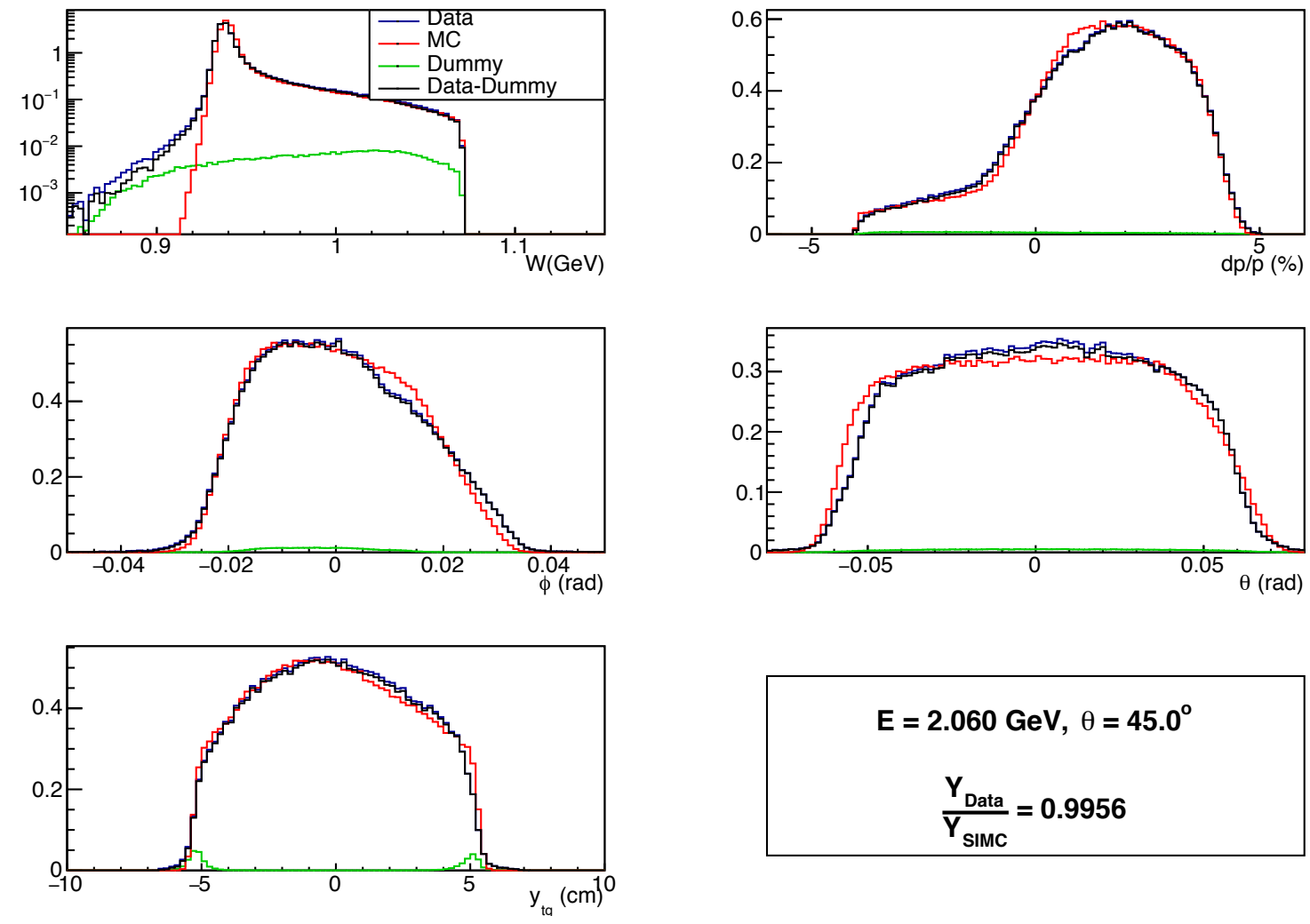

FIG. 7.2: Data yield compared to Monte Carlo yield of kinematics $b$ (see Table 6.1) .

The variables are in the target coordinate system and a pion threshold cut $W<1.07 \mathrm{GeV}$ is applied. The blue line shows the data before the dummy subtraction; the green line shows the dummy yield; the black line shows the data after the dummy subtraction; the red line shows the Monte Carlo yield. 

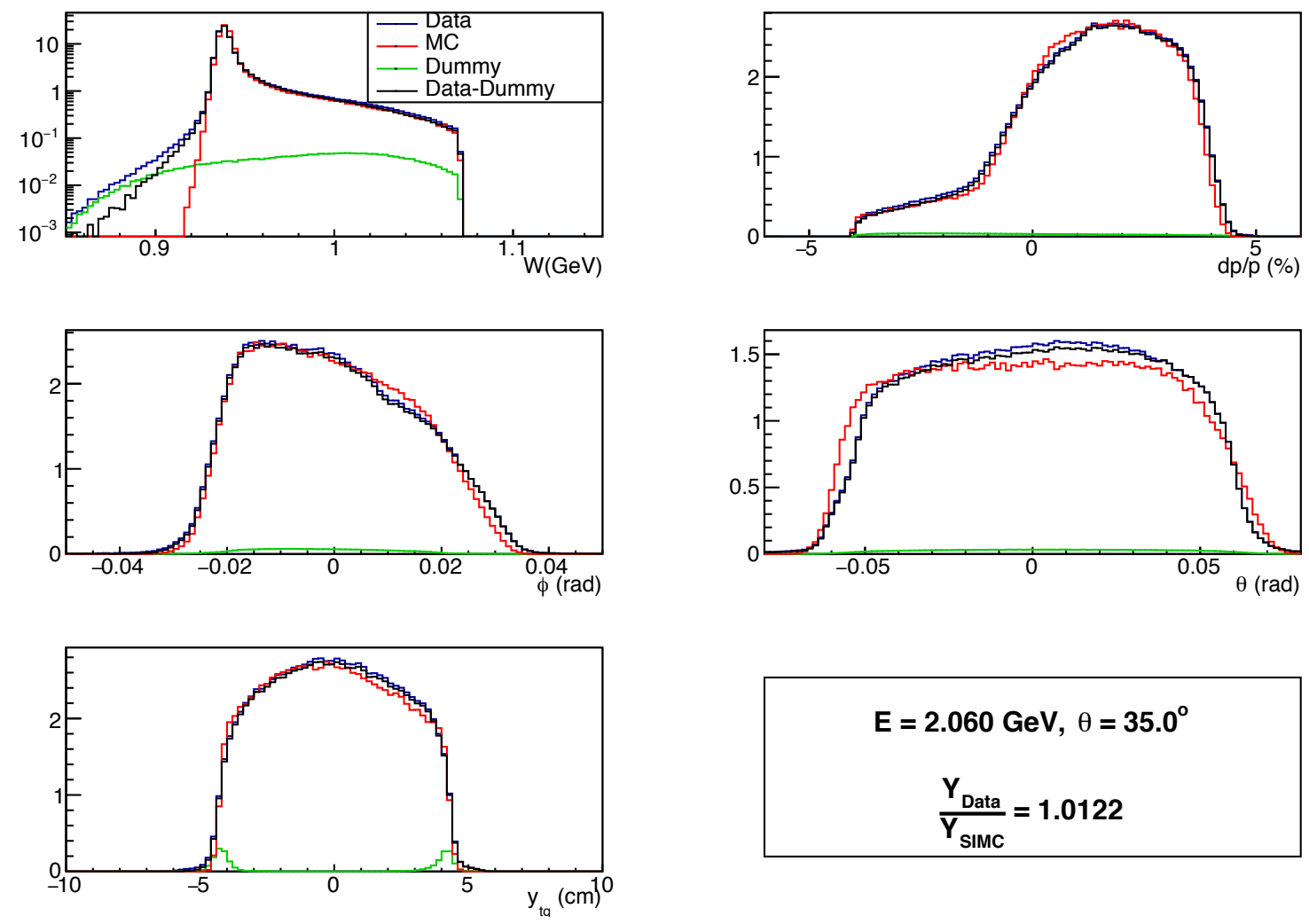

FIG. 7.3: Data yield compared to Monte Carlo yield of kinematics $c$ (see Table 6.1). The variables are in the target coordinate system and a pion threshold cut $W<1.07 \mathrm{GeV}$ is applied. The blue line shows the data before the dummy subtraction; the green line shows the dummy yield; the black line shows the data after the dummy subtraction; the red line shows the Monte Carlo yield. 

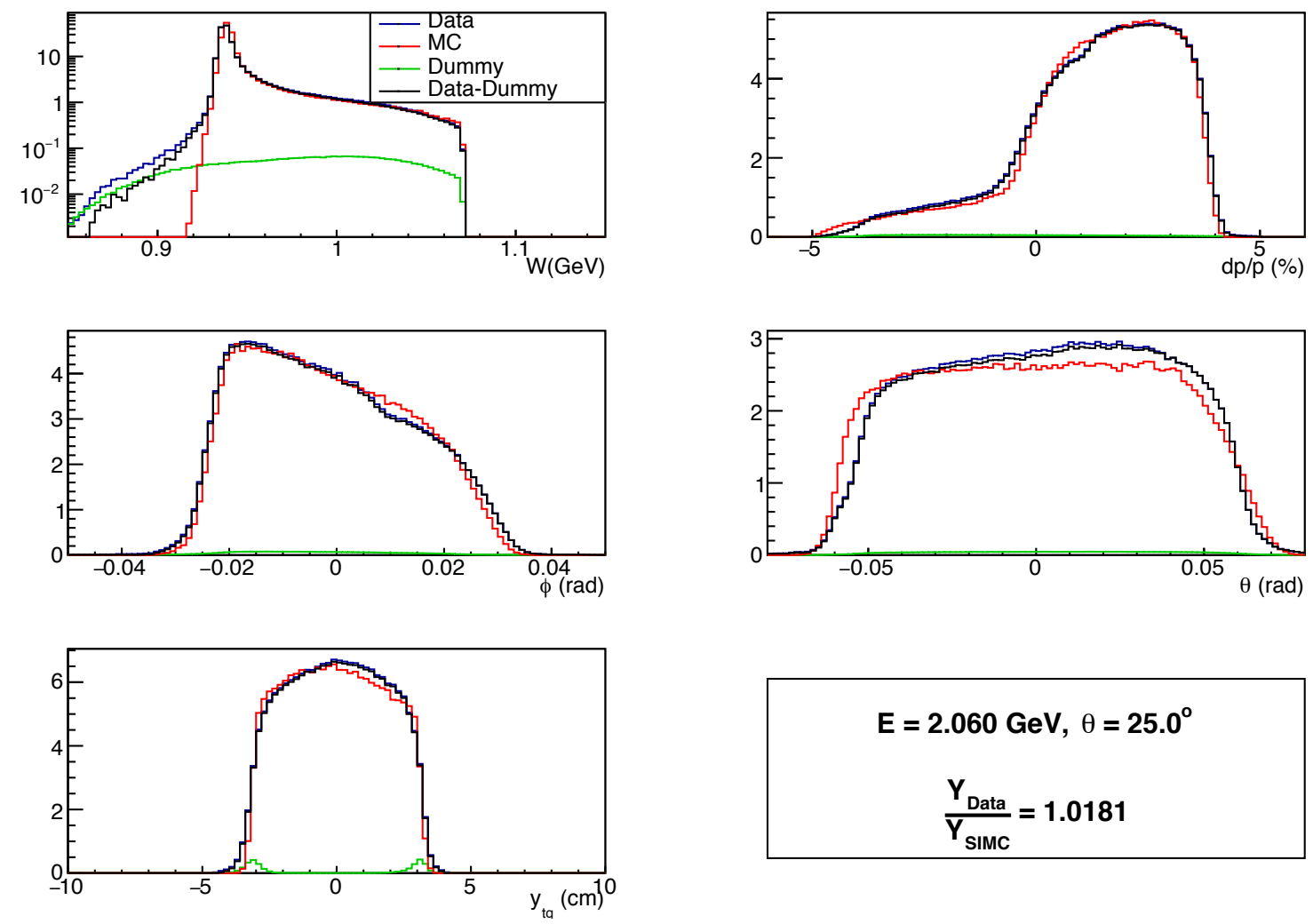

FIG. 7.4: Data yield compared to Monte Carlo yield of kinematics $d$ (see Table 6.1). The variables are in the target coordinate system and a pion threshold cut $W<1.07 \mathrm{GeV}$ is applied. The blue line shows the data before the dummy subtraction; the green line shows the dummy yield; the black line shows the data after the dummy subtraction; the red line shows the Monte Carlo yield. 
which is acceptable. A study of the Monte Carlo apertures and magnets' fields has been investigated to determine the uncertainty from the MC. However, Fig. 7.2 to Fig. 7.4 show that there are discrepancies for these kinematics between the experimental data and $\mathrm{MC}$, primarily in the out-plane-of angle $\theta$. Investigations have been made indicating that the discrepancy is mainly from the forward and backward optics matrices in the MC for the L-HRS (see Appendix ??). However, the kinematics is not very sensitive to $\theta$ and a conservative $4 \%$ uncertainty is applied to cover this discrepancy. The collaboration will continue the study to resolve this discrepancy in the future. The reduced cross sections obtained from the MC and data ratio method for each kinematics are listed in Table 7.1, where the uncertainties are dominated by the uncertainty from the discrepancy between the MC and experimental data.

TABLE 7.1: The elastic $e-p$ reduced cross section $\sigma_{R}$ for all the kinematics.

\begin{tabular}{|c|c|c|c|c|}
\hline$Q^{2}\left(\mathrm{GeV}^{2}\right)$ & Kinematics & $\epsilon$ & $Y / Y_{M C}$ & $\sigma_{R}$ \\
\hline 1.66 & $a$ & 0.624 & 0.995 & $(3.74 \pm 0.10) \times 10^{-2}$ \\
1.51 & $b$ & 0.671 & 0.996 & $(4.46 \pm 0.19) \times 10^{-2}$ \\
1.10 & $c$ & 0.793 & 1.012 & $(8.07 \pm 0.35) \times 10^{-2}$ \\
0.66 & $d$ & 0.895 & 1.018 & $(1.71 \pm 0.07) \times 10^{-1}$ \\
\hline
\end{tabular}

\subsection{Comparison with World Data}

The elastic $e-p$ cross sections for all of the kinematics have been obtained and a comparison is made between the experiment E12-07-108 results in the spring of 2015 with the world data. Fig. 7.5 presents the obtained cross sections by the and Fig. 7.6 shows the comparison, where $(d \sigma / d \Omega)_{1 \gamma, \text { dipole }}$ in the figure is the reduced cross section obtained with $G_{E p}=G_{D}$ and $G_{M p}=\mu_{p} G_{D}$. The figure shows that the cross sections from the experiment 
E12-07-108 are in a good agreement with previous measurements.

\section{JLab E012-07-108, $e-p$ elastic cross section}

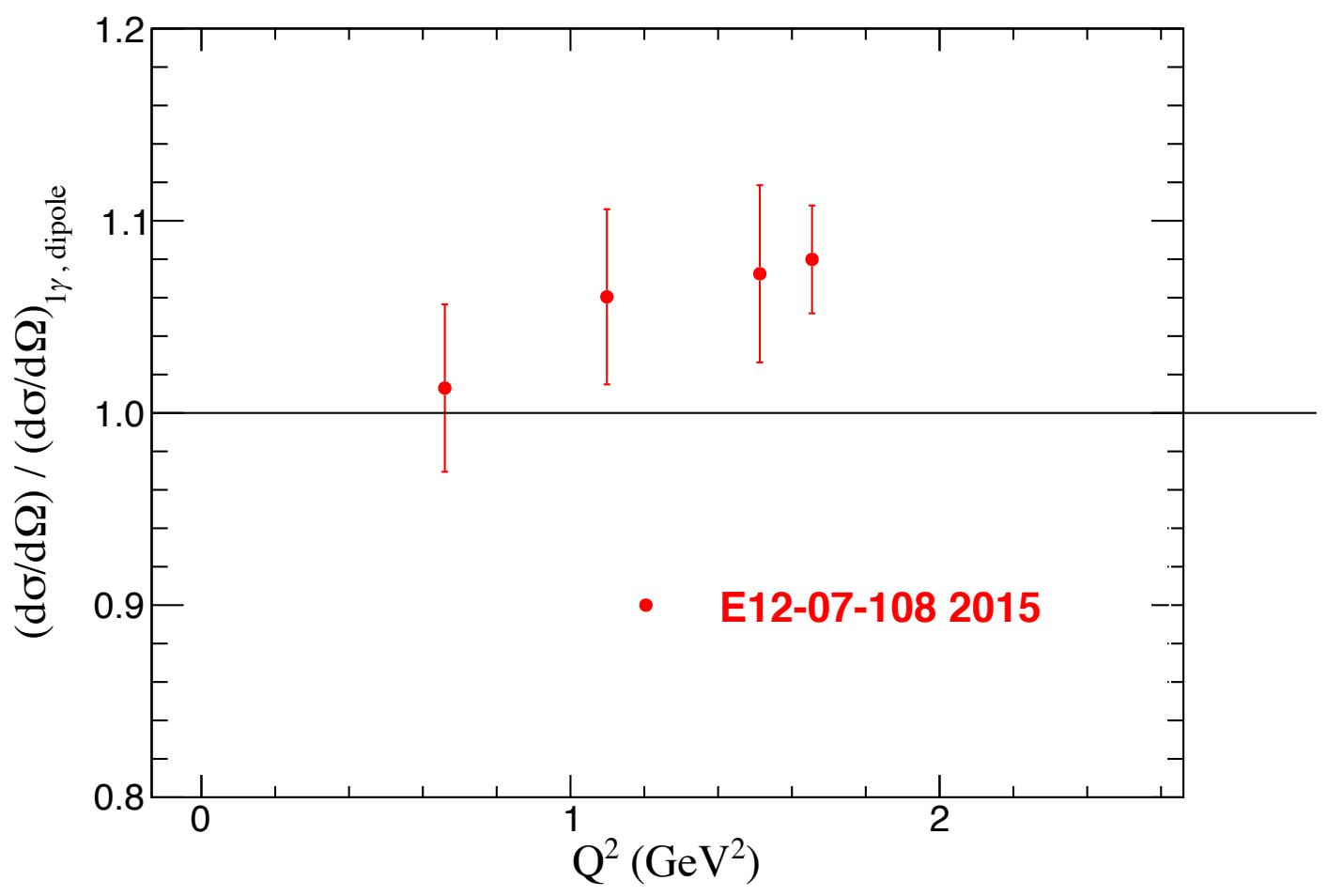

FIG. 7.5: The $e-p$ elastic cross sections obtained from the experiment E12-07-108 data in the spring of 2015 at $Q^{2}=0.66,1.10,1.51,1.65 \mathrm{GeV}^{2}$.

For the cross sections obtained by the experimental data in the spring of 2015, none of them has the same $Q^{2}$ and thus the Rosenbluth separation method is not applicable to extract the form factors. In Fig. 7.6, we notice that there are several previous measurements at $Q^{2}$ very close to $0.66 \mathrm{GeV}^{2}$, the lowest $Q^{2}$ of the experimental data in the spring of 2015. Fig. 7.7 shows the reduced cross sections obtained from these measurements and the spring 2015 experimental data. The previous measurements are scaled with a factor $R$, moving them to the same $Q^{2}$ as the experimental data. $R$ is calculated as follows:

$$
R=\frac{\sigma_{R}\left(Q_{E}^{2}\right)}{\sigma_{R}\left(Q_{M}^{2}\right)}=\frac{\epsilon G_{E p}^{2}\left(Q_{E}^{2}\right)+\tau G_{M p}^{2}\left(Q_{E}^{2}\right)}{\epsilon G_{E p}^{2}\left(Q_{M}^{2}\right)+\tau G_{M p}^{2}\left(Q_{M}^{2}\right)}
$$




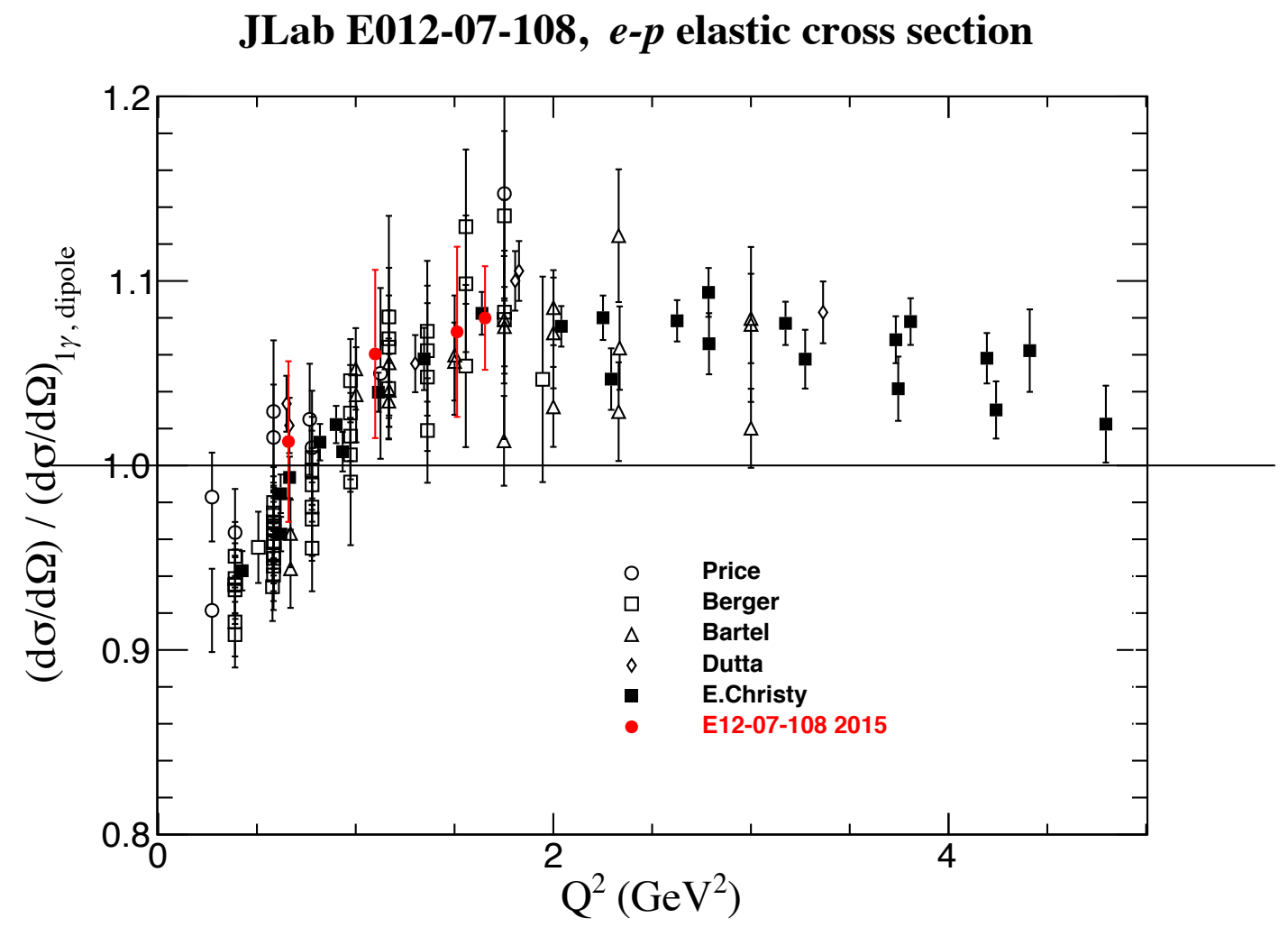

FIG. 7.6: Comparison of the $e-p$ elastic cross sections between the experiment E12-07-108 results and previous measurements. The cross sections obtained from experimental data are shown as the red points and previous measurements are black points, where $(d \sigma / d \Omega)_{1 \gamma, \text { dipole }}$ is the reduced cross section obtained with $G_{E p}=G_{D}$ and $G_{M p}=\mu_{p} G_{D}$. 
where $Q_{E}^{2}$ is the $Q^{2}$ of the experimental data and $Q_{M}^{2}$ is that of each previous measurement. In Fig. 7.6, $Q_{E}^{2}=0.660 \mathrm{GeV}^{2} . G_{E p}$ and $G_{M p}$ are calculated using Eq. 7.8 and 7.9. The Rosenbluth separation method can be applied and the form factors are obtained, $G_{M p} / \mu_{p} G_{D}=0.99$ and $G_{E p} / G_{D}=1.07$. The form factors are also extracted with $R$ calculated using the Bosted fit [82]. The difference between these two cross section models are very small, $\sim 0.03 \%$.

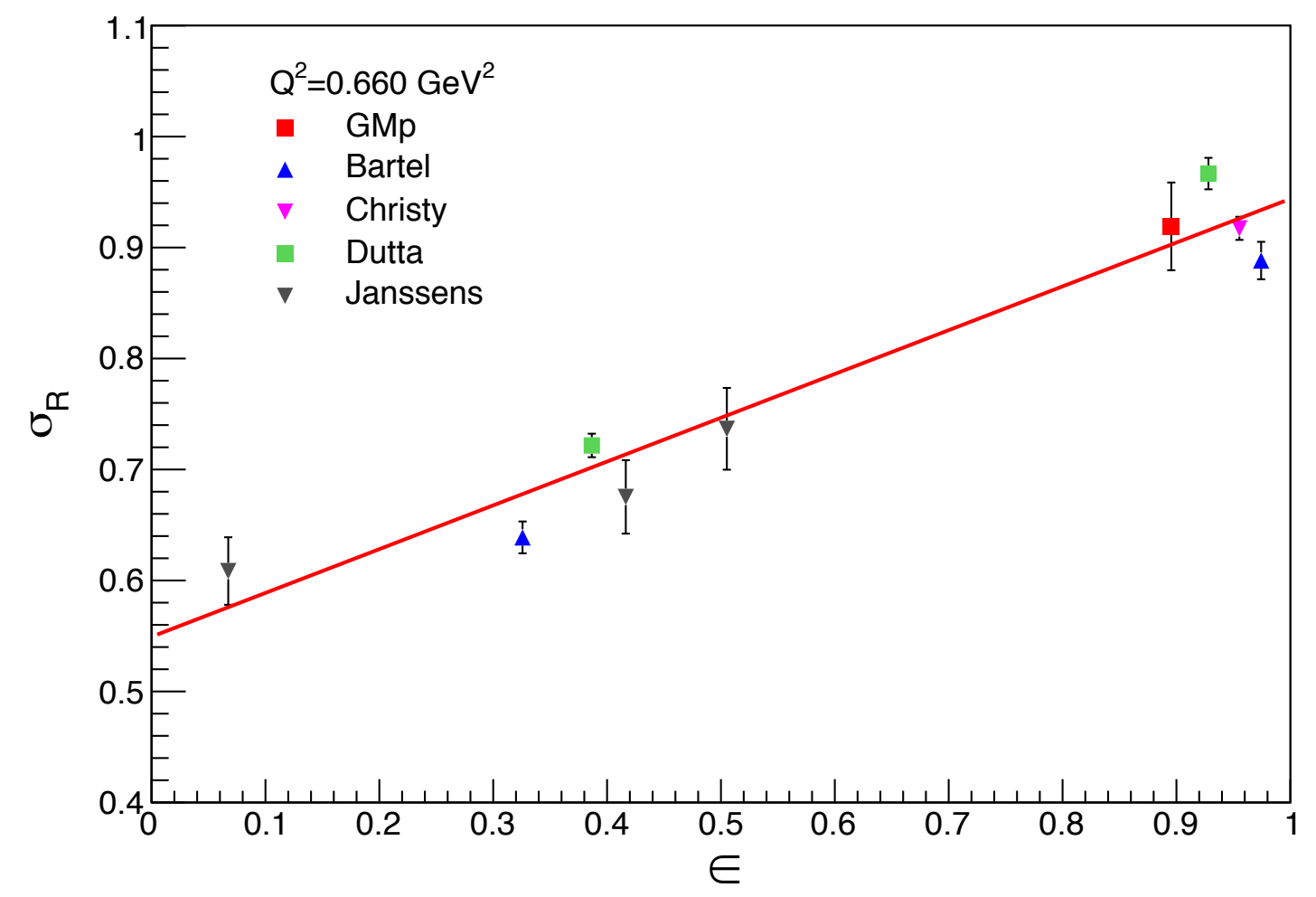

FIG. 7.7: The reduced elastic $e-p$ cross sections vs. $\epsilon$ obtained from previous measurements at $Q^{2}$ close to $0.66 \mathrm{GeV}^{2}$ and the experiment E12-07-108 data at $Q^{2}=0.660 \mathrm{GeV}^{2}$. Data of the previous measurements are from references $[17,11,83,12]$.

Similarly, the same procedure is followed for $Q^{2}=1.10 \mathrm{GeV}^{2}$ and the form factors are obtained, $G_{M p} / \mu_{p} G_{D}=1.00$ and $G_{E p} / G_{D}=1.14$.

Fig. 7.9 presents the comparison of the proton electric form factor $G_{E p}$ between the 


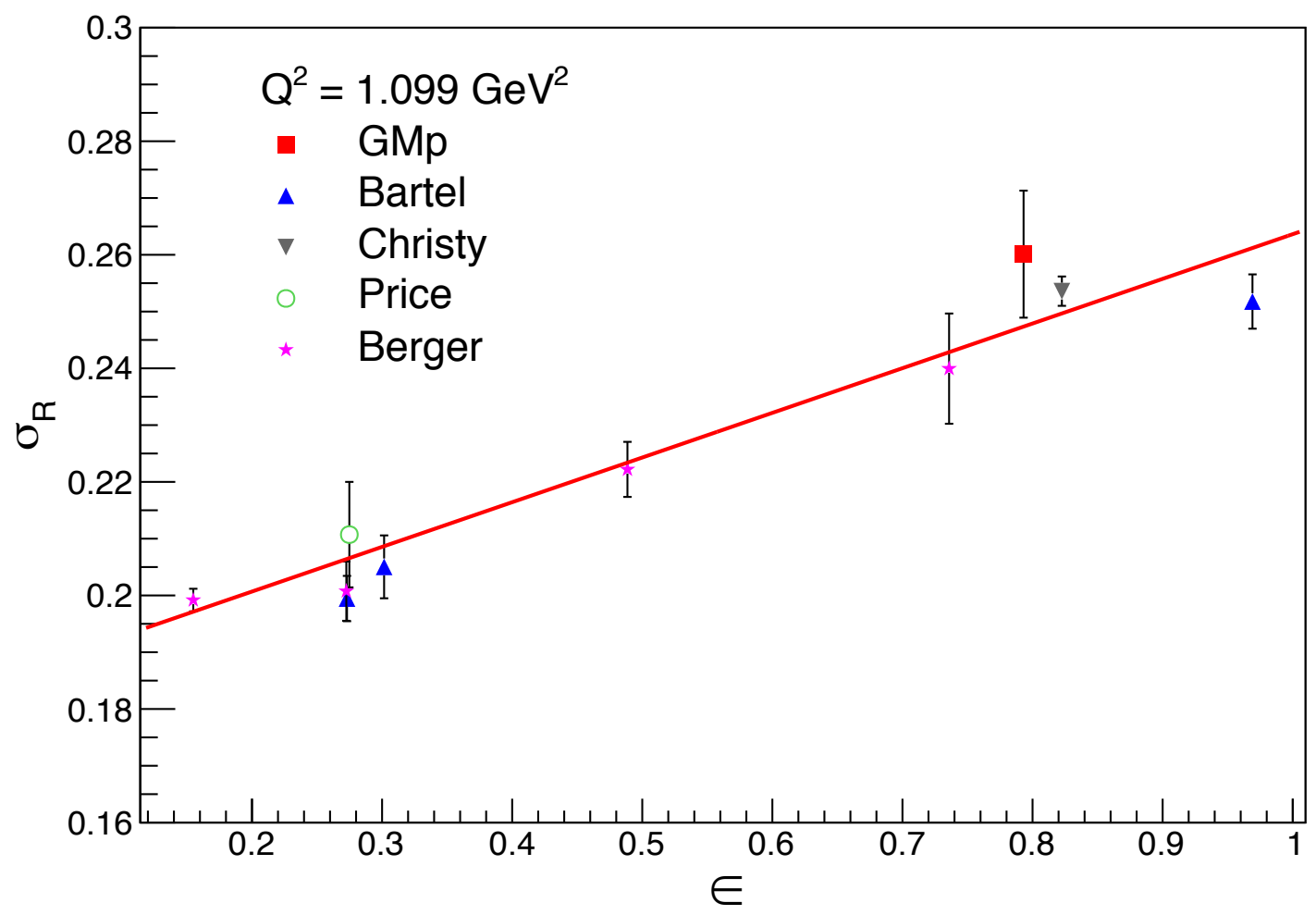

FIG. 7.8: The reduced elastic $e-p$ cross sections vs $\epsilon$ obtained from previous measurements at $Q^{2}$ close to $1.099 \mathrm{GeV}^{2}$ and the experimental data at $Q^{2}=1.099 \mathrm{GeV}^{2}$. Data of the previous measurements are from references $[17,11,13,9]$. 
world data and that obtained by combining the experiment E12-07-108 data and previous measurements. Fig. 7.10 shows the comparison of the proton magnetic form factor $G_{M p}$. For $Q^{2}=0.66 \mathrm{GeV}^{2}$, both $G_{E p}$ and $G_{M p}$ are in good agreement with the world data.

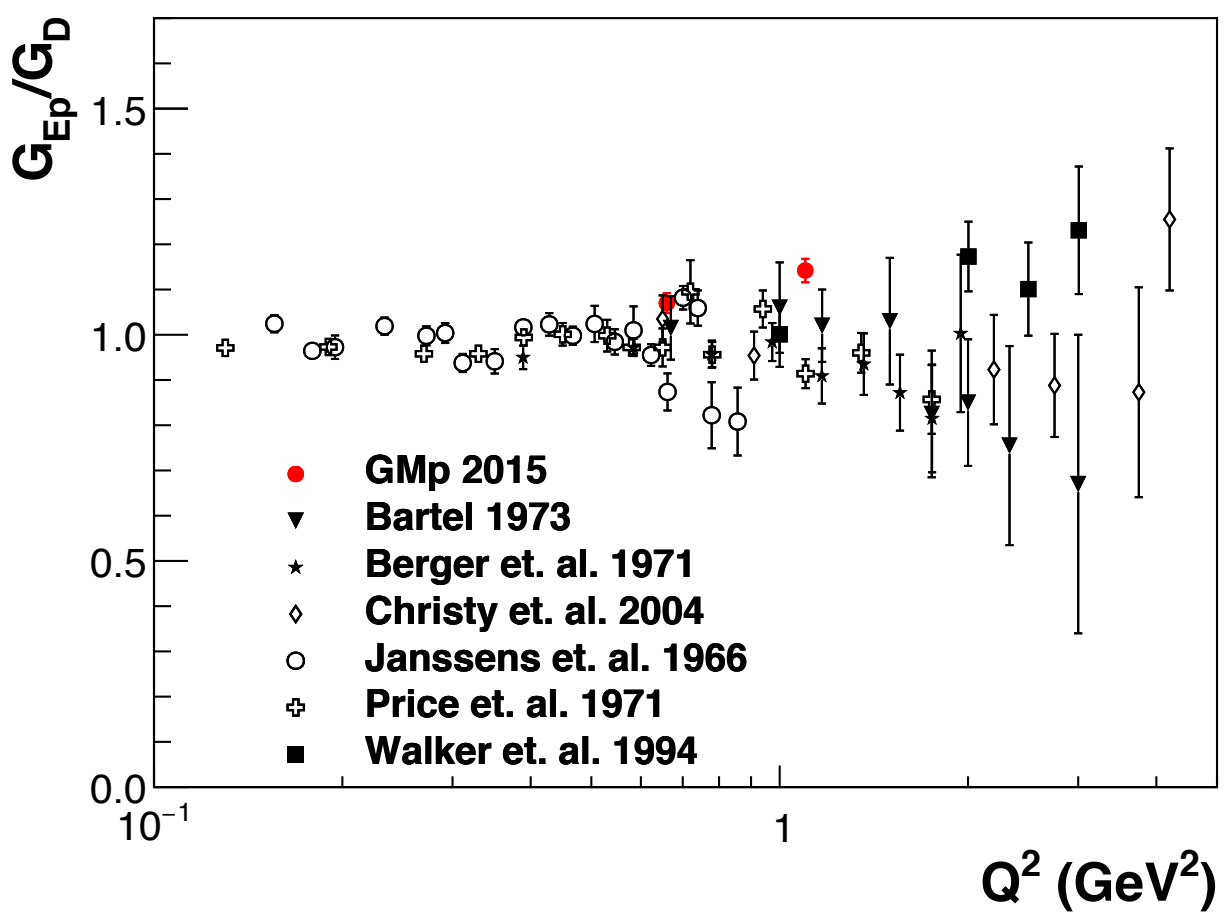

FIG. 7.9: The comparison of the proton electric form factor $G_{E p}$ between the world data and that obtained by combining the experimental data and previous measurements at similar $Q^{2}$. Data of the previous measurements are from references $[17,9,11,12,13,16]$.

\subsection{Conclusion}

The analysis of the experiment E12-07-108 data taken in the spring of 2015 shows that the elastic $e-p$ cross sections are in good agreement with the previous world data. Combining the cross sections obtained from the experiment E12-07-108 data and previous measurements gives reasonable proton electric and magnetic form factors, $G_{E p}$ and $G_{M p}$, using the Rosenbluth separation method. 


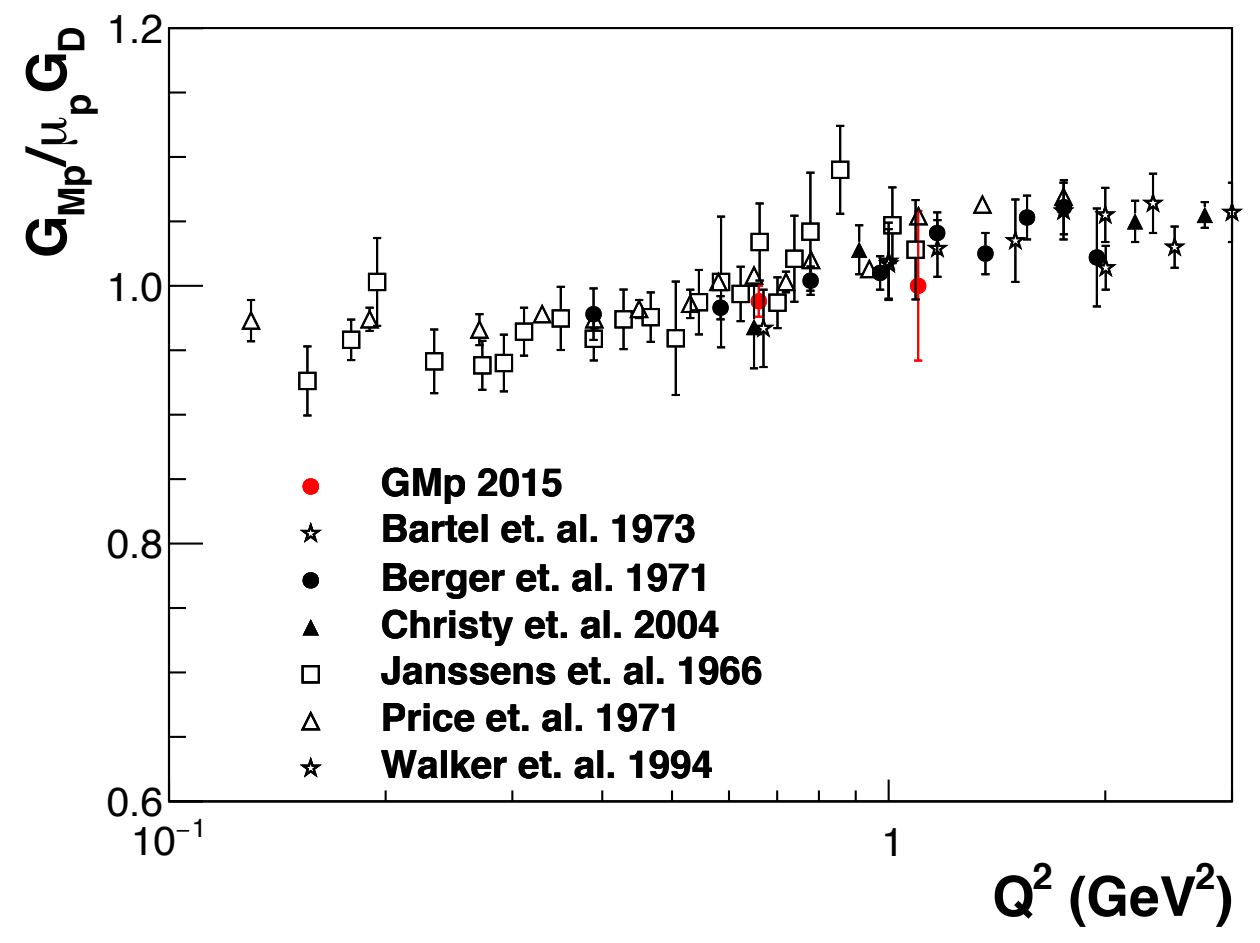

FIG. 7.10: The comparison of the proton electric form factor $G_{M p}$ between the world data and that obtained by combining the experiment E12-07-108 data and previous measurements at similar $Q^{2}$. Data of the previous measurements are from references $[17,9,11,12,13,16]$. 
The cross sections calculated using the Monte Carlo simulation and data yields ratio method are based on the cross section model used in the Monte Carlo simulation. The data taken in the spring of 2015 are located in the $Q^{2}$ range where lots of previous measurements have been performed. The good agreement between the calculated cross sections and the previous world data provides confidence for the analysis of the experiment E12-07-108 data at higher $Q^{2}$ Fig. 3.1, where only a few previous measurements exist with larger uncertainties.

The systematic uncertainties are all below $1 \%$ in this thesis except the MC uncertainty, which we believe is due to the not prefect match between the MC and spectrometer optics. For the data taken during 2016 spring and fall, the optics calibrations were improved due to more sieve holes and the presence of SOS quadrupole magnets. I have optimized the optics for the data in 2016 spring and fall. The MC and experimental data agree quite well over the acceptance with one-pass beam. For the 2016 data, we expect disagreement between MC and data cross-sections stemming from acceptance modeling to below $0.5 \%$. The final total systematic uncertainty for the cross section from the 2016 data is expected to be $2 \%$ or better.

For data at higher $Q^{2}=6-15 \mathrm{GeV}^{2}$ the E12-07-108 collaboration will present preliminary results in the summer of 2017. 
APPENDIX A

Diagrams of the tungsten sieve for GMp optics calibration 


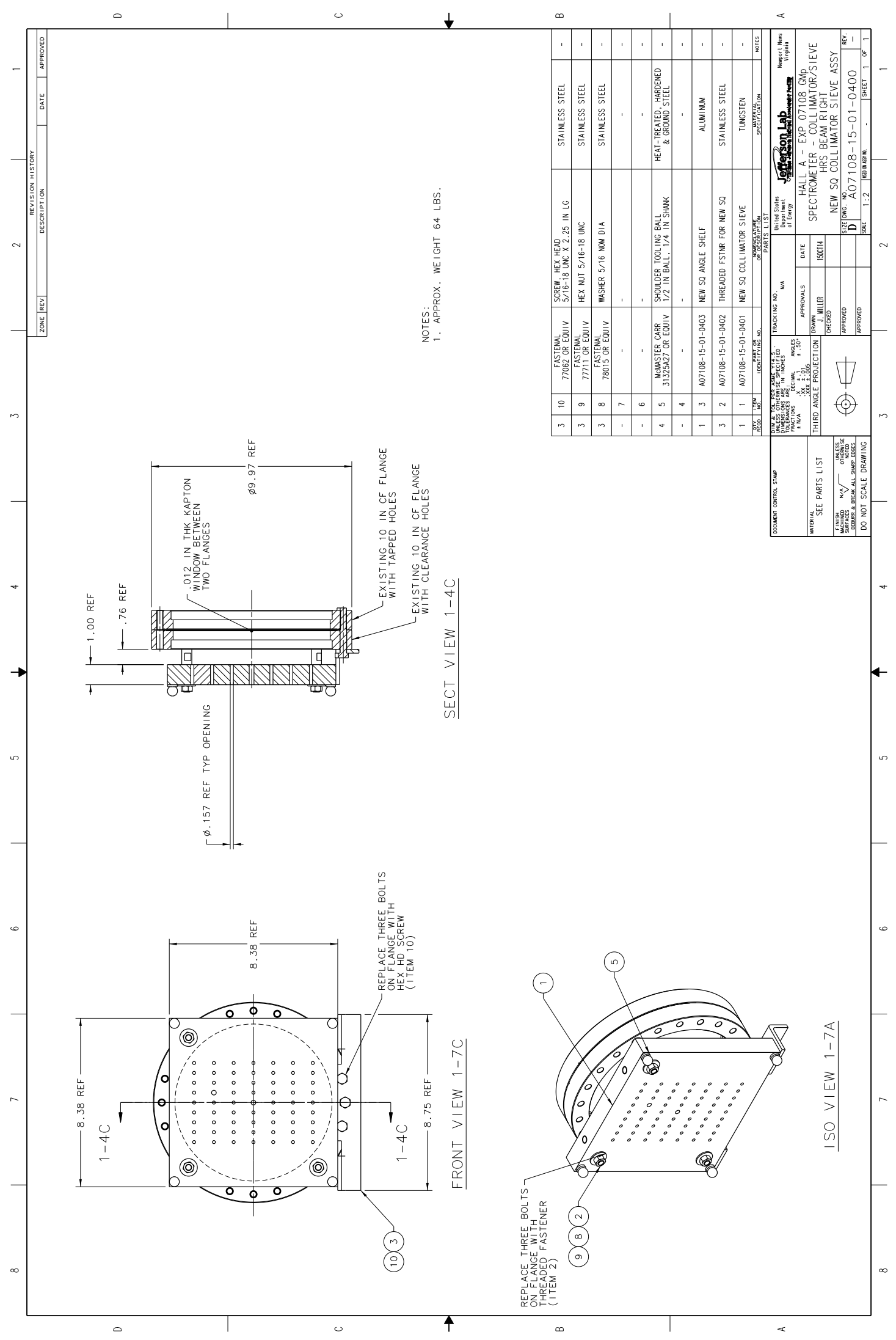




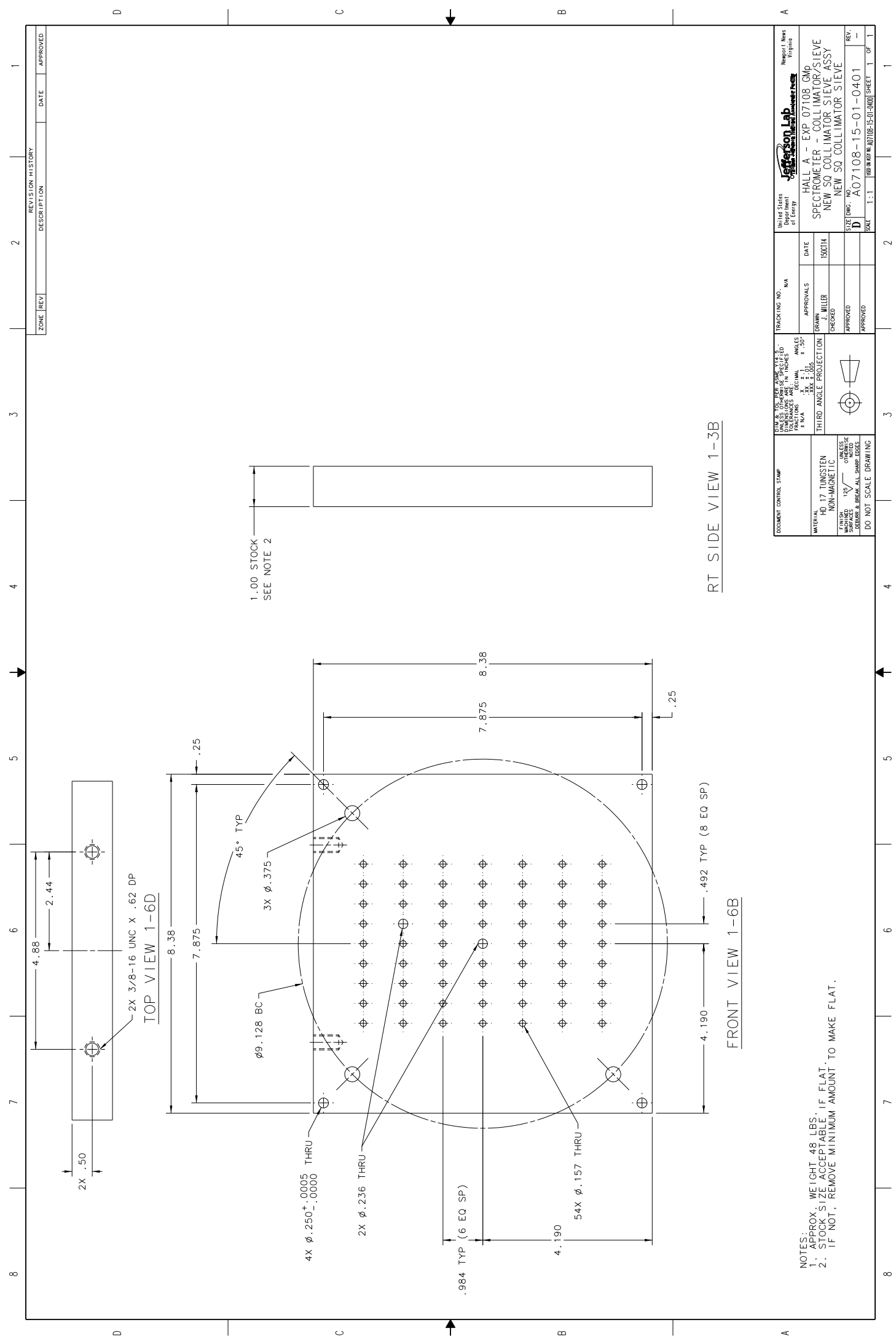




\section{APPENDIX B}

\section{Uncertainty}

\begin{tabular}{|c|c|c|}
\hline Quadrupole & Field strengh $\mathrm{F}$ & Ratio/Ratio $_{0}$ \\
\hline Q1 field*1.01 & $\mathrm{F}^{*} 1.01$ & $1+0.0009$ \\
\hline Q1 field*0.99 & $\mathrm{F}^{*} 0.99$ & $1-0.0026$ \\
\hline Q2 field*1.01 & $\mathrm{F}^{*} 1.01$ & $1-0.0329$ \\
\hline Q2 field*0.99 & $\mathrm{F}^{*} 0.99$ & $1+0.0341$ \\
\hline Q2 field*1.005 & $\mathrm{F}^{*} 1.005$ & $1-0.0183$ \\
\hline Q2 field*0.995 & $\mathrm{F}^{*} 0.995$ & $1+0.0160$ \\
\hline Q3 field*1.01 & $\mathrm{F}^{*} 1.01$ & $1-0.0023$ \\
\hline Q3 field*0.99 & $\mathrm{F}^{*} 0.99$ & $1+0.0018$ \\
\hline
\end{tabular}

TABLE B.1: The uncertainties calculated by the MC when the field strength is off the setting value for each quadrupole. Ratio ${ }_{0}$ is with no field strengths off. 


\begin{tabular}{|c|c|c|}
\hline Aperture Number & Aperture Displacement & Ratio/Ratio 0 \\
\hline 3 & position_x-1mm & 1.0030 \\
\hline 3 & position_x+1mm & 1.0031 \\
\hline 3 & position_y-1mm & 1.0032 \\
\hline 3 & position_y+1mm & 1.0028 \\
\hline 4 & position_x-1mm & 1.0013 \\
\hline 4 & position_x+1mm & 1.0035 \\
\hline 4 & position_y-1mm & 1.0023 \\
\hline 4 & position_y+1mm & 0.9993 \\
\hline 5 & position_x-1mm & 0.9991 \\
\hline 5 & position_x+1mm & 1.0029 \\
\hline 5 & position_y-1mm & 0.9995 \\
\hline 5 & position_y+1mm & 1.0007 \\
\hline 8 & position_x-1mm & 1.0001 \\
\hline 8 & position_x+1mm & 1.0018 \\
\hline 8 & position_y-1mm & 1.0030 \\
\hline 8 & position_y+1mm & 1.0038 \\
\hline 9 & position_x-1mm & 0.9989 \\
\hline 9 & position_x+1mm & 1.0029 \\
\hline 9 & position_y-1mm & 1.0005 \\
\hline 9 & position_y+1mm & 1.0013 \\
\hline 10 & position_x-1mm & 1.0003 \\
\hline 10 & position_x+1mm & 0.9993 \\
\hline 10 & position_y-1mm & 0.9988 \\
\hline 10 & position_y+1mm & 1.0024 \\
\hline 16 & position_x-1mm & 1.0010 \\
\hline 16 & position_x+1mm & 1.0027 \\
\hline 16 & position_y-1mm & 1.0013 \\
\hline 16 & position_y+1mm & 1.0002 \\
\hline 18 & position_x-1mm & 1.0038 \\
\hline 18 & position_x+1mm & 1.0019 \\
\hline
\end{tabular}

TABLE B.2: The uncertainties caused by the displacement of each aperture. Note $x$ and $y$ are in the target coordinate system and Ratio $_{0}$ is with no apertures are moved. 


\section{BIBLIOGRAPHY}

[1] R. C. Walker et al., Phys. Rev. D 49, 5671 (1994).

[2] A. V. Belitsky, X. Ji, and F. Yuan, Phys. Rev. Lett. 91, 092003 (2003).

[3] J. Huang, Ph.D. thesis, Massachusetts Institute of Technology (2012).

[4] CODA, https://coda.jlab.org.

[5] C. Perdrisat, V. Punjabi, and M. Vanderhaeghen, Prog. Part. Nucl. Phys. 59, 694 (2007), ISSN 0146-6410.

[6] A. I. Akhiezer and M. Rekalo, Sov. J. Part. Nucl. 4, 277 (1974), [Fiz. Elem. Chast. Atom. Yadra4,662(1973)].

[7] R. G. Arnold, C. E. Carlson, and F. Gross, Phys. Rev. C 23, 363 (1981).

[8] L. Andivahis et al., Phys. Rev. D50, 5491 (1994).

[9] C. Berger, V. Burkert, G. Knop, B. Langenbeck, and K. Rith, Phys. Lett. B 35, 87 (1971).

[10] F. Borkowski, G. Simon, V. Walther, and R. Wendling, Nucl. Phys. B 93, 461 (1975), ISSN 0550-3213.

[11] M. E. Christy et al., Phys. Rev. C 70, 015206 (2004). 
[12] T. Janssens, R. Hofstadter, E. B. Hughes, and M. R. Yearian, Phys. Rev. 142, 922 (1966).

[13] L. E. Price, J. R. Dunning, M. Goitein, et al., Phys. Rev. D4, 45 (1971).

[14] I. A. Qattan, J. Arrington, et al., Phys. Rev. Lett. 94, 142301 (2005).

[15] G. Simon, C. Schmitt, F. Borkowski, and V. Walther, Nucl. Phys. A 333, 381 (1980), ISSN 0375-9474.

[16] R. Walker, B. Filippone, et al., Phys. Rev. D 49, 5671 (1994), ISSN 1550-7998.

[17] W. Bartel, F. W. Busser, W. r. Dix, et al., Nucl. Phys. B58, 429 (1973).

[18] A. F. Sill, R. G. Arnold, P. E. Bosted, et al., Phys. Rev. D 48, 29 (1993).

[19] M. K. Jones, K. A. Aniol, F. T. Baker, et al. (The Jefferson Lab Hall A Collaboration), Phys. Rev. Lett. 84, 1398 (2000).

[20] V. Punjabi, C. F. Perdrisat, et al. (Jefferson Lab Hall A Collaboration), Phys. Rev. C 71, 055202 (2005).

[21] O. Gayou, K. A. Aniol, et al. (Jefferson Lab Hall A Collaboration), Phys. Rev. Lett. 88, $092301(2002)$.

[22] P. G. Blunden, W. Melnitchouk, and J. A. Tjon, Phys. Rev. Lett. 91, 142304 (2003).

[23] P. A. M. Guichon and M. Vanderhaeghen, Phys. Rev. Lett. 91, 142303 (2003).

[24] Y.-C. Chen, A. Afanasev, S. J. Brodsky, C. E. Carlson, and M. Vanderhaeghen, Phys. Rev. Lett. 93, 122301 (2004).

[25] A. V. Afanasev, S. J. Brodsky, C. E. Carlson, Y.-C. Chen, and M. Vanderhaeghen, Phys. Rev. D 72, 013008 (2005). 
[26] P. G. Blunden, W. Melnitchouk, and J. A. Tjon, Phys. Rev. C 72, 034612 (2005).

[27] S. Kondratyuk, P. G. Blunden, W. Melnitchouk, and J. A. Tjon, Phys. Rev. Lett. 95, $172503(2005)$.

[28] J. Arrington, W. Melnitchouk, and J. A. Tjon, Phys. Rev. C 76, 035205 (2007).

[29] F. Iachello, A. Jackson, and A. Lande, Phys. Lett. B B 43, 191 (1973), ISSN 03702693.

[30] M. Gari and W. Krümpelmann, Zeit. f. Phys. A 322, 689 (1985), ISSN 0939-7922.

[31] M. F. Gari and W. Krümpelmann, Phys. Rev. D 45, 1817 (1992).

[32] E. L. Lomon, Phys. Rev. C 64, 035204 (2001).

[33] X. Zhan, Ph.D. thesis, Massachusetts Institute of Technology (2010).

[34] G. Hohler, E. Pietarinen, I. Sabba Stefanescu, F. Borkowski, G. G. Simon, V. H. Walther, and R. D. Wendling, Nucl. Phys. B114, 505 (1976).

[35] P. Mergell, U. G. Meissner, and D. Drechsel, Nucl. Phys. A596, 367 (1996), hep-ph/ 9506375.

[36] M. A. Belushkin, H.-W. Hammer, and U.-G. Meißner, Phys. Rev. C 75, 035202 (2007).

[37] W. Brooks and J. Lachniet, Nuclear Physics A 755, 261 (2005), ISSN 0375-9474, proceedings of the 10th International Conference on the Structure of Baryons.

[38] N. Isgur and G. Karl, Phys. Rev. D 18, 4187 (1978).

[39] A. De Rújula, H. Georgi, and S. L. Glashow, Phys. Rev. D 12, 147 (1975).

[40] P. A. M. Dirac, Rev. Mod. Phys. 21, 392 (1949). 
[41] P. L. Chung and F. Coester, Phys. Rev. D 44, 229 (1991).

[42] M. R. Frank, B. K. Jennings, and G. A. Miller, Phys. Rev. C 54, 920 (1996).

[43] F. Cardarelli, E. Pace, G. Salm, and S. Simula, Physics Letters B 357, 267 (1995), ISSN 0370-2693.

[44] F. Cardarelli and S. Simula, Phys. Rev. C 62, 065201 (2000).

[45] S. Boffi, L. Y. Glozman, W. Klink, W. Plessas, M. Radici, and R. F. Wagenbrunn, Eur. Phys. J. A14, 17 (2002), hep-ph/0108271.

[46] M. Gockeler et al., PoS LAT2006, 120 (2006), hep-lat/0610118.

[47] V. B. Berestetsky and M. V. Terentev, Sov. J. Nucl. Phys. 24, 547 (1976), [Yad. Fiz. $24,1044(1976)]$

[48] V. B. Berestetsky and M. V. Terentev, Sov. J. Nucl. Phys. 25, 347 (1977), [Yad. Fiz. $25,653(1977)]$.

[49] H. J. Melosh, Phys. Rev. D 9, 1095 (1974).

[50] F. Schlumpf, Phys. Rev. D 47, 4114 (1993).

[51] F. Schlumpf, J. Phys. G. 20, 237 (1994).

[52] G. A. Miller and M. R. Frank, Phys. Rev. C 65, 065205 (2002).

[53] G. A. Miller, Phys. Rev. C 66, 032201 (2002).

[54] S. Capstick and N. Isgur, Phys. Rev. D 34, 2809 (1986).

[55] L. Y. Glozman, Z. Papp, W. Plessas, K. Varga, and R. F. Wagenbrunn, Phys. Rev. C 57, 3406 (1998). 
[56] L. Y. Glozman, W. Plessas, K. Varga, and R. F. Wagenbrunn, Phys. Rev. D 58, 094030 (1998).

[57] A. J. R. Puckett, Ph.D. thesis, Massachusetts Institute of Technology (2010).

[58] P. N. Kirk, M. Breidenbach, et al., Phys. Rev. D 8, 63 (1973).

[59] C. W. Leemann, D. R. Douglas, and G. A. Krafft, Ann. Rev. Nucl. Part. Sci. 51, 413 (2001).

[60] J. Alcorn et al., Nucl. Instrum. Meth. A522, 294 (2004).

[61] X. Zheng, Ph.D. thesis, Massachusetts Institute of Technology (2002).

[62] W. Barry et al., Beam position measurement in the CEBAF recirculating linacs by use of pseudorandom pulse sequences (1990), JLab-TN-90-246 (unpublished).

[63] W. Barry et al., Basic noise considerations for CEBAF beam position monitors (1991), JLab-TN-91-087 (unpublished).

[64] K. Unser, IEEE Trans. Nucl. Sci. 28, 2344 (1981).

[65] K. G. Fissum et al., Nucl. Instrum. Meth. A474, 108 (2001).

[66] W. Bertozzi, M. V. Hynes, C. P. Sargent, C. Creswell, P. C. Dunn, A. Hirsch, M. Leitch, B. Norum, F. N. Rad, and T. Sasanuma, Nucl. Instrum. Meth. 141, 457 (1977).

[67] K. I. Blomqvist et al., Nucl. Instrum. Meth. A403, 263 (1998).

[68] M. Iodice et al., Nucl. Instrum. Meth. A411, 223 (1998).

[69] EPICS, https://www.jlab.org/accel/documents/epics_doc2.html. 
[70] N. Liyanage, "C Optimizer", Jefferson Lab Hall A Tech (unpublished). Note 02-012 C (2002).

[71] E. Jensen, Ph.D. thesis, The College of William and Mary (2013).

[72] I. Racheck et al., New amplifier/discriminator cards for multiwire drift chambers, https://userweb.jlab.org/ brads/Manuals/Hardware/nanometric-n277_ cards/MAD_card-technote.pdf.

[73] ANALYZER, http://hallaweb.jlab.org/podd/.

[74] N. Makins, Ph.D. thesis, Massachusettes Institute of Technology (1994).

[75] T. O’Neill, Ph.D. thesis, California Institute of Technology (1994).

[76] J. Arrington, W. Melnitchouk, and J. A. Tjon, Phys. Rev. C 76, 035205 (2007).

[77] M. Berz, NSCL Technical Report MSUCL-977 (1995).

[78] L. W. Mo and Y. S. Tsai, Rev. Mod. Phys. 41, 205 (1969).

[79] R. Walker, Ph.D. thesis, California Institute of Technology (1989).

[80] R. Ent, B. W. Filippone, N. C. R. Makins, R. G. Milner, T. G. O’Neill, and D. A. Wasson, Phys. Rev. C 64, 054610 (2001).

[81] I. A. Qattan, Ph.D. thesis, Massachusetts Institute of Technology (2012).

[82] P. E. Bosted et al., Phys. Rev. Lett. 68, 3841 (1992).

[83] D. Dutta, van Westrum, et al., Phys. Rev. C 68, 064603 (2003). 